Portland State University

PDXScholar

Summer 8-12-2015

\title{
Investigation in to the Effect of Spin Locking on Contrast Agent Relaxivity
}

Julian Saunders Haigh

Portland State University

Follow this and additional works at: https://pdxscholar.library.pdx.edu/open_access_etds

Part of the Diagnosis Commons

Let us know how access to this document benefits you.

\section{Recommended Citation}

Haigh, Julian Saunders, "Investigation in to the Effect of Spin Locking on Contrast Agent Relaxivity" (2015). Dissertations and Theses. Paper 2496.

https://doi.org/10.15760/etd.2493

This Dissertation is brought to you for free and open access. It has been accepted for inclusion in Dissertations and Theses by an authorized administrator of PDXScholar. Please contact us if we can make this document more accessible: pdxscholar@pdx.edu. 
Investigation in to the Effect of Spin Locking on Contrast Agent Relaxivity

by

Julian Saunders Haigh

A dissertation submitted in partial fulfillment of the requirements for the degree of

Doctor of Philosophy

in

Chemistry

Dissertation Committee:

Mark Woods, Chair

Andrea Goforth

David Peyton

Andrew Rice

William Rooney

Portland State University

2015 
(C) 2015 Julian Saunders Haigh 


\begin{abstract}
The current trend in magnetic resonance imaging (MRI) is towards higher external magnetic field strengths $\left(B_{0}\right)$ to take advantage of increased sensitivity and signal to noise ratio (SNR). Unfortunately, as $B_{0}$ increases the effectiveness (relaxivity) of clinical gadolinium $\left(\mathrm{Gd}^{3+}\right)$-based contrast agents $(\mathrm{CAs})$ administered to enhance image contrast is significantly reduced. Excellent soft tissue contrast can be generated with current agents despite their non-optimum relaxivities but necessitates large doses. The limits of detection of a CA at high $B_{0}$ fields can be lowered by recovering the lost relaxivity and is a pre-requisite to the goal of molecular imaging in which CAs are bound to biomarkers of pathology that exist at very low concentrations. Traditional methods for increasing the detectability of CAs have focused on optimizing critical parameters identified from the Solomon-Bloembergen-Morgan (SBM) theory that affect relaxivity. Gains in relaxivity with these methods to date have been modest and are far from the theoretical maximum possible. Although researchers continue to investigate novel complexes that provide improved relaxivities, any such complex would require a lengthy and costly approval process with the U.S. Food and Drug Administration (FDA). Therefore, a method that affords improved relaxivities of current clinically approved CAs, particularly at high $B_{0}$ fields, that could be adopted into clinical practice rapidly, is of great interest.
\end{abstract}

Spin locking is a nuclear magnetic resonance (NMR) technique that was introduced for imaging in 1985 , but has received very little attention in combination with $\mathrm{Gd}^{3+}$-based CAs. The technique employs a low power long duration radiofrequency (RF) pulse $\left(B_{1}\right)$ 
parallel to the net magnetization in the $\mathrm{x}, \mathrm{y}$-plane. This locks the magnetization into lower precessional frequencies around an "effective" field $\left(B_{\text {eff }}\right)$ that is reduced with respect to $B_{0}$ but maintains the high field advantages required for imaging. When considered in the rotating frame, longitudinal relaxation of the magnetization against $B_{\text {eff }}$ exhibits shorter time constants $\left(T_{1 \rho}\right)$ expected at these lower precessional frequencies. This leads to higher relaxivities, which has implications for increasing CA detectability.

The experiments described herein show that rotating frame longitudinal relaxivities $\left(r_{1} \rho\right)$ for current clinical $\mathrm{Gd}^{3+}$-based CAs are essentially independent of the strength of the spin lock pulse $\left(\gamma B_{1}\right)$ as predicted by theory. This result is important because it allows the value of $\gamma B_{1}$ to be neglected when comparing $r_{1 \rho}$ of $\mathrm{Gd}^{3+}$-based CAs across several $B_{0}$ fields. The magnetic field dependence of $r_{1 \rho}$ for all clinical agents showed that relaxivity, lost by moving to higher fields, could be "recovered" and that $r_{1 \rho}$ was sensitive to the rotational correlation time constant $\left(\tau_{\mathrm{R}}\right)$ of the agent. Using high molecular weight Nanoassembled capsules (NACs) containing a $\mathrm{Gd}^{3+}$-based $\mathrm{CA}$ to probe this finding further, we were able to generate relaxivities at high field up to an order of magnitude greater than clinical agents at current imaging fields. These are beyond anything previously reported, or likely to be, with current techniques. Finally, we demonstrated that by spin locking $\mathrm{Mn}^{2+}$ agents, relaxivities at high field increased by a factor of $\sim 30$ than without spin locking, due to their larger dependence on scalar coupling. These findings show the potential of spin locking to increase detection limits dramatically at high field and are an exciting development towards the goal of molecular imaging. 
I dedicate this thesis to the memory of my father-in-law who passed away suddenly towards the end of writing of this thesis. He will be sorely missed.

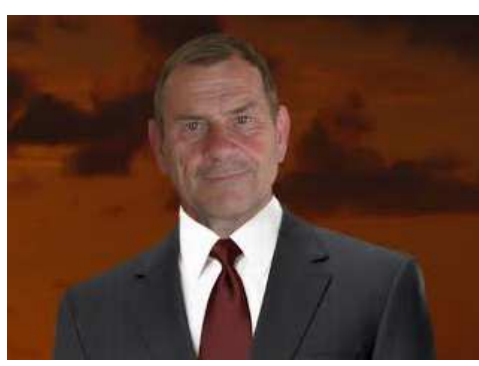

Professor Selwyn Wright

$(1934-2015)$ 


\section{Acknowledgements}

I would like to take this opportunity to thank the following:

Prof. Mark Woods for all his support, encouragement and assistance throughout the course of this research.

Annah Farashishiko for the provision of NACs that contributed to the success of this work.

Dr Benjamin Webber, Katherine Payne, Jacqueline Slack, Guadalupe Camacho and Lauren Rust for their contributions to the development of this work through feedback from listening to much iteration of my research presentations in group meetings.

Prof. Mauro Botta for the provision of several NMRD profiles modeling rotating frame relaxivities in particular.

Robert Jensen for his swift response to scheduling requests for access to the high field NMR spectrometers and for providing his expertise when required.

Dr Thomas Barbara for his discussions on power attenuation calculations.

My wife, Justine, and son, Daniel for all their support and patience throughout my graduate education.

Professors Andrea Goforth, David Peyton, Andrew Rice and William Rooney for their time and feedback as members of the dissertation committee.

Portland State University, Oregon Health \& Sciences University and Oregon Nanoscience and Microtechnology Institute for financial support. 


\section{Table of Contents}

$\begin{array}{lr}\text { Abstract } & \text { i } \\ \text { Dedication } & \text { iii } \\ \text { Acknowledgements } & \text { iv } \\ \text { List of Tables } & \text { xii } \\ \text { List of Figures } & \text { xiv } \\ \text { List of Equations } & \text { xvii } \\ \text { List of Terms and Abbreviations } & \text { xx } \\ \text { Chapter } 1 . \quad \text { Introduction } & 1\end{array}$

1.1. Magnetic resonance imaging: a critical imaging mode of the past, present and future 1

1.1.1. The rise to popularity of MRI 1

1.1.2. Modern day MRI and its comparison with other imaging modalities 2

1.1.3. The future of clinical MRI 3

1.2. Nuclear magnetic resonance (NMR) theory 3

1.2.1. The Zeeman effect 4

1.2.2. Signal detection 8

1.2.3. Longitudinal relaxation 9

$\begin{array}{ll}\text { 1.2.4. Transverse relaxation } & 15\end{array}$

1.2.5. Fourier transformation, $T_{1}$ and $T_{2}$ determination $\quad 17$

1.3. Inherent soft tissue contrast and image weighting 19

1.4. $\mathrm{Gd}^{3+}$-based contrast agents for soft tissue contrast enhancement 24 
1.4.1. Classification of clinically approved $\mathrm{Gd}^{3+}$-based CA

1.4.2. Stabilization of $\mathrm{Gd}^{3+}$-based CAs 28

1.4.3. Mechanism of action of $\mathrm{Gd}^{3+}$-based CAs 29

1.4.4. Contributions to the PRE effect 30

1.5. Traditional methods for optimization of relaxivity: A discussion of SBM $\begin{array}{ll}\text { theory } & 32\end{array}$

1.5.1. Motives for increasing relaxivity 32

1.5.2. SBM parameters for optimizing relaxivity 34

1.5.3. Hydration state (q) 34

1.5.4. Inner sphere water proton relaxation time constant $\left(\mathrm{T}_{1 \mathrm{M}}\right)$

1.5.5. Rotational correlation time constant $\left(\tau_{\mathrm{R}}\right) \quad 36$

1.5.6. Water residence lifetime $\left(\tau_{M}\right) \quad 37$

1.5.7. Electron relaxation time constants $\left(\tau_{\mathrm{S} 1}\right.$ and $\left.\tau_{\mathrm{S} 2}\right) \quad 38$

1.6. Spin locking for recovering lost relaxivity 38

1.6.1. Theory of spin locking 39

1.6.2. Determination of rotating frame longitudinal relaxation time $\begin{array}{ll}\text { constants }\left(\mathrm{T}_{1 \rho}\right) & 39\end{array}$

1.7. Scope of this work: Determining the effect of a spin locking pulse on relaxivity

1.7.1. Effect of pulse sequence parameters on $T_{1}$ and $T_{1 \rho}$ time constants

1.7.2. Effect of spin lock pulse strength $\left(\gamma B_{1}\right)$ on longitudinal relaxation rate constants $\left(R_{1 \rho}\right)$ 
1.7.3. A comparison of laboratory $\left(r_{1}\right)$ and rotating $\left(r_{1 \rho}\right)$ frame longitudinal relaxivities for $\mathrm{q}=1$ clinical $\mathrm{Gd}^{3+}$-based CAs

1.7.4. Effect of rotational correlation time $\left(\tau_{R}\right)$ on rotating frame longitudinal relaxivity $\left(r_{1 \rho}\right)$

1.7.5. Effect of hydration number (q) on rotating frame longitudinal relaxivity $\left(r_{1 \rho}\right)$

Chapter 2. Effect of pulse sequence parameters on longitudinal $\left(T_{1}\right)$ and rotating $\left(T_{1} \rho\right)$ frame time constants

2.1. Establishing pertinent pulse sequence parameters with potential to effect reliability of fit of relaxation data

2.1.1. Relaxation delay (RD) time

2.1.2. Pulse delay $(\tau)$ times

2.1.3. Number of data points

2.2. Establishment of ideal pulse sequence parameter values

2.3. Other parameters and general considerations

2.3.1. Sample temperature, volume and position

2.3.2. Pulse angles

2.3.3. Offset frequency

2.3.4. Number of scans

2.3.5. Receiver gain, detection mode and fitting equations

2.4. Determination of natural variance (standard deviation) from replicate analysis

2.5. Effect of pulse sequence parameters on the reliability of fit of relaxation data 
2.5.3. Number of data points

$\begin{array}{lll}\text { 2.6. Recommendations } & 57\end{array}$

2.6.1. Relaxation delay (RD) times $\quad 59$

$\begin{array}{lll}\text { 2.6.2. } & \text { Pulse delay times } & 61\end{array}$

2.6.3. Number of data points $\quad 62$

$\begin{array}{lll}\text { 2.7. Conclusions } & 62\end{array}$

Chapter 3. Effect of spin lock field strength $\left(\gamma B_{1}\right)$ on the rotating frame longitudinal $\begin{array}{ll}\text { relaxation rate constant }\left(r_{1} \rho\right) & 64\end{array}$

3.1. Establishing the $\gamma B_{1}$ independency of $R_{1 \rho} \quad 64$

3.2. Modulation of the strength of the spin lock field $\left(\gamma B_{1}\right) \quad 66$

3.3. Effect of $\gamma B_{1}$ on $R_{1 \rho}$ at low $B_{0}$ field strengths 68

3.4. Effect of $\gamma B_{1}$ on $R_{1 \rho}$ at high $B_{0}$ field strengths 70

3.5. A comparison between spin locking and CPMG with short pulse delay times $\quad 73$

$\begin{array}{ll}\text { 3.6. Conclusions } & 77\end{array}$

Chapter 4. A comparison of laboratory $\left(r_{1}\right)$ and rotating $\left(r_{1} \rho\right)$ frame longitudinal relaxivities for $\mathrm{q}=1$ clinical $\mathrm{Gd}^{3+}$-based CAs $\quad 78$

4.1. Establishing the potential benefits of spin locking on relaxivity 78

4.2. Comparison of $r_{1}$ and $r_{1 \rho}$ for linear and macrocyclic $\mathrm{q}=1$ clinical $\mathrm{Gd}^{3+}-$ $\begin{array}{ll}\text { based CAs } & 80\end{array}$ 
4.3. A comparison of $r_{1 \rho}$ for linear and macrocyclic chelates to simulated theory

4.4. A comparison of $r_{1}$ and $r_{1 \rho}$ for bulky q $=1$ clinical $\mathrm{Gd}^{3+}$-based CAs

4.5. Summary of relaxivity changes

4.6. Conclusions

Chapter 5. Effect of rotational correlation time $\left(\tau_{\mathrm{R}}\right)$ on rotating frame longitudinal relaxivity $\left(r_{1 \rho}\right)$

5.1. Establishing the potential for increasing relaxivity by spin locking slow tumbling nano-encapsulated $\mathrm{Gd}^{3+}$ chelates.

5.2. Nanoassembled capsules as a model system for investigating the effect of $\tau_{R}$ on $\mathrm{r}_{1 \rho}$

5.3. Mechanism of action of nanoassembled capsules (NACs) incorporating GdDOTP ${ }^{5-}$

5.4. Effect of NAC design parameters on relaxivity

5.5. Effect of $\gamma B_{1}$ on $R_{1 \rho}$ for NACs at high $B_{0}$ field strengths

5.6. A comparison of laboratory $\left(r_{1}\right)$ and rotating $\left(r_{1 \rho}\right)$ frame NMRD for NACs

5.6.1. Effect of $\tau_{\mathrm{R}}$ on $r_{1}$ as a function of $B_{0}$

5.6.2. Effect of $\tau_{\mathrm{R}}$ on $r_{1 \rho}$ as a function of $B_{0}$

5.7. Summary of relaxivity changes

5.8. Conclusions

Chapter 6. Effect of hydration number $(q)$ on rotating frame longitudinal relaxivity (rip)

6.1. Potential applications of spin locking with hydration numbers $(\mathrm{q}) \neq 1$ 
6.2. Low molecular weight chelates of $\mathrm{Gd}^{3+}$ and $\mathrm{Mn}^{2+}$ as systems for investigating the effect of q on $r_{1 \rho}$

6.3. Effect of $\gamma B_{1}$ on $R_{1 \rho}$ for $\mathrm{MnCl}_{2}$ at low $B_{0}$ field strengths

6.4. A comparison of laboratory $\left(r_{1}\right)$ and rotating $\left(r_{1 \rho}\right)$ frame NMRD for low molecular weight $\mathrm{Gd}^{3+}$ and $\mathrm{Mn}^{2+}$ agents as a function of hydration number (q) 118

6.4.1. A comparison of $r_{1}$ and $r_{1 \rho}$ for low molecular weight non-bulky $\mathrm{Gd}^{3+}$ complexes

6.4.2. A comparison of $r_{1}$ and $r_{1 \rho}$ for low molecular weight $\mathrm{Mn}^{2+}$ complexes

6.5. Conclusions

Chapter 7. Experimental section

7.1. Reagents and solvents

7.2. Instrumentation

7.3. Pulse sequences

7.3.1. Inversion recovery pulse sequence

7.3.2. Carr-Purcell-Meiboom-Gill (CPMG) pulse sequence

7.3.3. Spin locking pulse sequence

7.4. $\quad$ Fitting

7.5. Contrast media stock solution preparations

7.5.1. Clinical $\mathrm{Gd}^{3+}$-based contrast agents

7.5.2. $\mathrm{GdCl}_{3}$

7.5.3. $\mathrm{MnCl}_{2}$ 
7.6. Working solution preparations and experimental parameters

7.6.1. Chapter 2. Effect of pulse sequence parameters on longitudinal $\left(T_{1}\right)$ and rotating $\left(T_{1 \rho}\right)$ frame time constants

7.6.2. Chapter 3. Effect of spin lock field strength $\left(\gamma B_{1}\right)$ on the rotating frame longitudinal relaxation rate constant $\left(R_{1} \rho\right)$

7.6.3. Chapter 4. A comparison of laboratory $\left(r_{1}\right)$ and rotating $\left(r_{1} \rho\right)$ frame longitudinal relaxivities for $\mathrm{q}=1$ clinical $\mathrm{Gd}^{3+}$-based CAs

7.6.4. Chapter 5. Effect of rotational correlation time $\left(\tau_{R}\right)$ on rotating frame longitudinal relaxivity $\left(r_{1} \rho\right)$

7.6.5. Chapter 6. Effect of hydration state (q) on rotating frame longitudinal relaxivity $\left(r_{1} \rho\right)$

APPENDIX: $T_{1}$ and $T_{1 \rho}$ time curves at $1.41 \mathrm{~T}(60 \mathrm{MHz})$ and $37^{\circ} \mathrm{C}$ (appendix to chapter 


\section{List of Tables}

Table 1.1: Physical properties of $\mathrm{Gd}^{3+}$-based CAs studied

Table 2.1: Suggested "ideal" pulse sequence parameter values

Table 2.2: $T_{1}$ and $T_{1 \rho}$ measurement repeatability data

Table 2.3: Effect of relaxation delay times on $T_{1}$ and $T_{1 \rho}$ values

Table 2.4: Effect of pulse delay times on $T_{1}$ and $T_{1 \rho}$ values

Table 2.5: Effect of number of data points on $T_{1}$ and $T_{1 \rho}$ values

Table 2.6: Recommended pulse sequence parameter values

Table 3.1: Effect of pulse delay time on $T_{2}$

Table 4.1: $r_{1}$ and $r_{1}$ NMRD data for clinical linear and macrocyclic $\mathrm{q}=1 \mathrm{CAs}$ (low field, $25^{\circ} \mathrm{C}$ )

Table 4.2: $r_{1}$ and $r_{1 \rho}$ NMRD data for clinical linear and macrocyclic $\mathrm{q}=1$ CAs (low field, $37^{\circ} \mathrm{C}$ )

Table 4.3: $r_{1}$ and $r_{1 \rho}$ NMRD data for clinical linear and macrocyclic $\mathrm{q}=1 \mathrm{CAs}$ (high field, $25^{\circ} \mathrm{C}$ )

Table 4.4: $r_{1}$ and $r_{1 \rho}$ NMRD data for clinical linear and macrocyclic $\mathrm{q}=1 \mathrm{CAs}$ (high field, $37^{\circ} \mathrm{C}$ )

Table 4.5: $r_{1}$ and $r_{1 \rho}$ NMRD data for clinical bulky q $=1$ CAs (low field)

Table 4.6: $r_{1}$ and $r_{1 \rho}$ NMRD data for clinical bulky q $=1$ CAs (high field) 
Table 6.1: $r_{1}$ and $r_{1 \rho}$ NMRD data for low molecular weight complexes of $\mathrm{Gd}^{3+}$ 


\section{List of Figures}

Figure 1.1: Influence of $B_{0}$ on a collection of proton nuclei 8

Figure 1.2: Influence of a $90^{\circ} \mathrm{RF} B_{1}$ pulse on net longitudinal magnetization $\quad 10$

Figure 1.3: Inversion recovery pulse timing diagram and course of magnetization 14

Figure 1.4: CPMG pulse timing diagram and course of magnetization 16

Figure 1.5: Summary of $T_{1}$ relaxation time constant determination 18

Figure 1.6: Summary of $T_{2}$ relaxation time constant determination 19

Figure 1.7: Spin echo pulse timing diagram for weighted imaging 20

Figure 1.8: Effect of image weighting on contrast 23

Figure 1.9: Distribution and excretion pathways of clinical $\mathrm{Gd}^{3+}$-based CAs 25

Figure 1.10: Structures of class 1 (ECF) agents 26

Figure 1.11: Structure of class 2 (ECF/liver) agents 27

Figure 1.12: Structure of class 3 (blood pool) agent 27

Figure 1.13: Macrocyclic and linear parent ligands for clinical $\mathrm{Gd}^{3+}$-based CAs 29

Figure 1.14: Schematic of the spheres of water that contribute to relaxivity 31

Figure 1.15: Laboratory frame NMRD profiles of Dotarem and an optimized gadoterate

chelate 33

Figure 1.16: Simulated effect of $\tau_{\mathrm{R}}$ and $\tau_{\mathrm{M}}$ on relaxivity for a generic $\mathrm{Gd}^{3+}$ chelate $\quad 36$

Figure 1.17: Spin Locking pulse timing diagram and course of magnetization $\quad 40$ 
Figure 1.18: Summary of $T_{1 \rho}$ relaxation time constant determination

Figure 2.1: $T_{1}$ and $T_{1} \rho$ measurement repeatability data

Figure 2.2: Effect of relaxation delay times on $T_{1}$ time curves $\left(25^{\circ} \mathrm{C}\right)$

Figure 2.3: Effect of relaxation delay times on $T_{1 \rho}$ time curves $\left(25^{\circ} \mathrm{C}\right)$

Figure 2.4: Effect of first pulse delay times on $T_{1}$ and $T_{1 \rho}$ time curves $\left(25^{\circ} \mathrm{C}\right)$

Figure 2.5: Effect of last pulse delay times on $T_{1}$ and $T_{1 \rho}$ time curves $\left(25^{\circ} \mathrm{C}\right)$

Figure 2.6: Effect of number of data points on $T_{1}$ and $T_{1 \rho}$ time curves $\left(25^{\circ} \mathrm{C}\right)$

Figure 3.1: Dependence of $R_{1 \mathrm{p}}$ on $\gamma B_{1}$ (low field)

Figure 3.2: Dependence of $R_{1 \mathrm{p}}$ on $\gamma B_{1}$ (high field)

Figure 3.3: Sample raw data for the high field $\gamma B_{1}$ dependence figure

Figure 3.4: Dependence of $T_{2}$ on pulse delay time

Figure 4.1: $r_{1}$ and $r_{1} \rho$ NMRD profiles for linear and macrocyclic $\mathrm{q}=1$ clinical $\mathrm{Gd}^{3+}$ based CAs

Figure 4.2: Sample relaxivity $\left(r_{1}\right)$ plots for the macrocyclic class of clinical agents

Figure 4.3: Simulated versus observed $r_{1 \rho}$ NMRD data for Magnevist and Dotarem

Figure 4.4: $r_{1}$ and $r_{1 \rho}$ NMRD profiles for bulky $\mathrm{q}=1$ clinical $\mathrm{Gd}^{3+}$-based CAs

Figure 4.5: Summary of the \% change in relaxivity by spin locking clinical agents 96

Figure 5.1: Simulated inner sphere contribution to $r_{1}$ and $r_{1 \rho}$ NMRD of a theoretically “optimized" gadoterate chelate versus $r_{1}$ NMRD of non-optimized gadoterate 
Figure 5.2: Cartoon of the spheres of water contributing to relaxivity for GdDOTP ${ }^{5-} 101$

Figure 5.3: Cartoon of a GdDOTP ${ }^{5-} \mathrm{NAC}$

Figure 5.4: SEM of GdDOTP ${ }^{5-}$ NACs

Figure 5.5: Dependence of $R_{1 \mathrm{p}}$ on $\gamma B_{1}$ of NACs (high field)

Figure 5.6: $r_{1}$ and $r_{1 \rho}$ NMRD profiles of GdDOTP ${ }^{5-}$ NACs versus free chelate

Figure 5.7: Summary of the \% change in relaxivity by spin locking NACs

Figure 6.1: Ligands for the preparation of low molecular weight $\mathrm{Gd}^{3+}$ and $\mathrm{Mn}^{2+}$ chelates with a variety of hydration states

Figure 6.2: Dependence of $R_{1 \mathrm{p}}$ on $\gamma B_{1}$ for $\mathrm{MnCl}_{2}$ (low field)

Figure 6.3: NMRD profiles of several low molecular weight chelates of $\mathrm{Gd}^{3+}$ with varying hydration states

Figure 6.4: NMRD profiles of several low molecular weight chelates of $\mathrm{Mn}^{2+}$ with varying hydration states

Figure 6.5: NMRD profiles of aqueous transition metal ions from literature 


\section{List of Equations}

Equation 1.1: Relationship between spin angular momentum and spin quantum number 4

Equation 1.2: Projection of the spin angular momentum vector along the z-axis

Equation 1.3: Proportionality between magnetic moment and spin angular momentum 5

Equation 1.4: Energy of spin states generated by Zeeman splitting

Equation 1.5: Redefined energy of spin states for a magnetic field along the z-axis 6

Equation 1.6: Difference in energy between two spin states

Equation 1.7: Linear frequency of EM radiation corresponding to energy gap

Equation 1.8: The Boltzmann distribution of spins

Equation 1.9: The Larmor frequency

Equation 1.10: Derivation of the general solution to the integrated first order rate law 12

Equation 1.11: Exponential recovery equation that describes the return of the $\mathrm{z}$ component of net magnetization towards equilibrium along the $\mathrm{z}$ axis after a $90^{\circ}$ pulse 13

Equation 1.12: Exponential recovery equation that describes the return of the $\mathrm{z}$ component of net magnetization towards equilibrium along the $\mathrm{z}$ axis after a $180^{\circ}$ pulse 13

Equation 1.13: Exponential decay equation that describes the loss of transverse relaxation from the $\mathrm{x}, \mathrm{y}$-plane and return to its equilibrium value after a $90^{\circ}$ pulse

Equation 1.14: Macroscopic transverse magnetization components of the FID in the $x, y-$ plane as a function of time 
Equation 1.15: Fourier transformation equation for mathematical transformation of signal from the time domain to frequency domain

Equation 1.16: Signal intensity equation for the spin echo weighting pulse sequence 21

Equation 1.17: Laboratory frame longitudinal relaxivity

Equation 1.18: Observed laboratory frame longitudinal relaxation rate constant

Equation 1.19: Inner sphere contribution to the observed laboratory frame longitudinal relaxation rate constant

Equation 1.20: Dependence of the inner sphere laboratory frame longitudinal relaxation rate constant

Equation 1.21: Dependence of the laboratory frame longitudinal and transverse correlation time constant

Equation 1.22: Dependence of the laboratory frame longitudinal electron spin relaxation time constant

Equation 1.23: Dependence of the laboratory frame transverse electron spin relaxation time constant

Equation 1.24: Exponential decay equation that describes the loss of transverse relaxation from the $\mathrm{x}, \mathrm{y}$-plane in the presence of a spin lock pulse and return to its equilibrium value after a $90^{\circ}$ excitation pulse

Equation 3.1: Dependence of the rotating frame longitudinal relaxation rate constant 64

Equation 3.2: The Rabi frequency

Equation 3.3: Condition for which $R_{1 \mathrm{r}}$ should be independent of $\gamma B_{1}$ in the presence of $\mathrm{Gd}^{3+}$ ions 
Equation 3.4: Dependence of $R_{1 \rho}$ in the presence of $\mathrm{Gd}^{3+}$ ions

Equation 3.5: Dependence of $r_{1 \rho}$ in the presence of $\mathrm{Gd}^{3+}$ ions

Equation 4.1: Dependence of the inner sphere laboratory frame longitudinal relaxation rate constant

Equation 6.1: Low power limit dependence of the rotating frame longitudinal relaxation rate constant 


\section{List of Terms and Abbreviations}

\begin{tabular}{|c|c|}
\hline $\mathrm{BBB}$ & Blood brain barrier \\
\hline$B_{\text {eff }}$ & Effective external magnetic field \\
\hline BSA & Bovine serum albumin \\
\hline CAs & Contrast agents \\
\hline CNR & Contrast to noise ratio \\
\hline CSF & Cerebrospinal fluid \\
\hline $\mathrm{CT}$ & Computed tomography \\
\hline DLS & Dynamic light scattering \\
\hline DOTA & $\begin{array}{l}2,2 \text { ',2' ',2','-(1,4,7,10-tetraazacyclododecane-1,4,7,10- } \\
\text { tetrayl)tetraacetate }\end{array}$ \\
\hline DOTP & $\begin{array}{l}\text { 1, 4, 7, 10-tetra-azacyclododecane- } N, N^{\prime}, N^{\prime \prime}, N^{\prime \prime \prime}- \\
\text { tetrakis(methylenephosphonic acetate) }\end{array}$ \\
\hline DTPA & 2,2',2",2"',2"'"-(1,2-ethanediylnitrilo)pentaacetate \\
\hline $\mathrm{ECF}$ & Extracellular fluid \\
\hline EDTA & 2,2',2",2"'-(1,2-ethanediyldinitrilo)tetraacetate \\
\hline EU & European union \\
\hline FDA & Food and Drug Administration \\
\hline FID & Free induction decay \\
\hline $\mathrm{Gd}^{3+}$-based CAs & Trivalent Gadolinium based contrast agent \\
\hline$\gamma B_{1}$ & Spin lock pulse strength \\
\hline
\end{tabular}




\begin{tabular}{|c|c|}
\hline HSA & Human serum albumin \\
\hline $\mathrm{ICF}$ & Intracellular fluid \\
\hline ICP-AES & Inductively coupled plasma-atomic emission spectroscopy \\
\hline M & Net magnetization \\
\hline $\mathrm{MeCN}$ & Methyl cyanide (Acetonitrile) \\
\hline $\mathrm{M}_{0}$ & Equilibrium magnetization \\
\hline $\mathrm{M}_{\mathrm{L}}$ & Net longitudinal magnetization \\
\hline $\mathrm{M}_{\mathrm{T}}$ & Net transverse magnetization \\
\hline MR & Magnetic resonance \\
\hline MRI & Magnetic resonance imaging \\
\hline MW & Molecular weight \\
\hline NACs & Nanoassembled capsules \\
\hline NMR & Nuclear magnetic resonance \\
\hline NMRD & Nuclear magnetic relaxation dispersion \\
\hline NPs & Nanoparticles \\
\hline PAH & Polyallylamine hydrochloide \\
\hline PBS & Phosphate buffered saline \\
\hline PD & Proton density \\
\hline PET & Positron emission tomography \\
\hline ppm & Parts per million \\
\hline PRE & Paramagnetic relaxation enhancement \\
\hline$r_{1}$ & Laboratory frame relaxivity \\
\hline
\end{tabular}




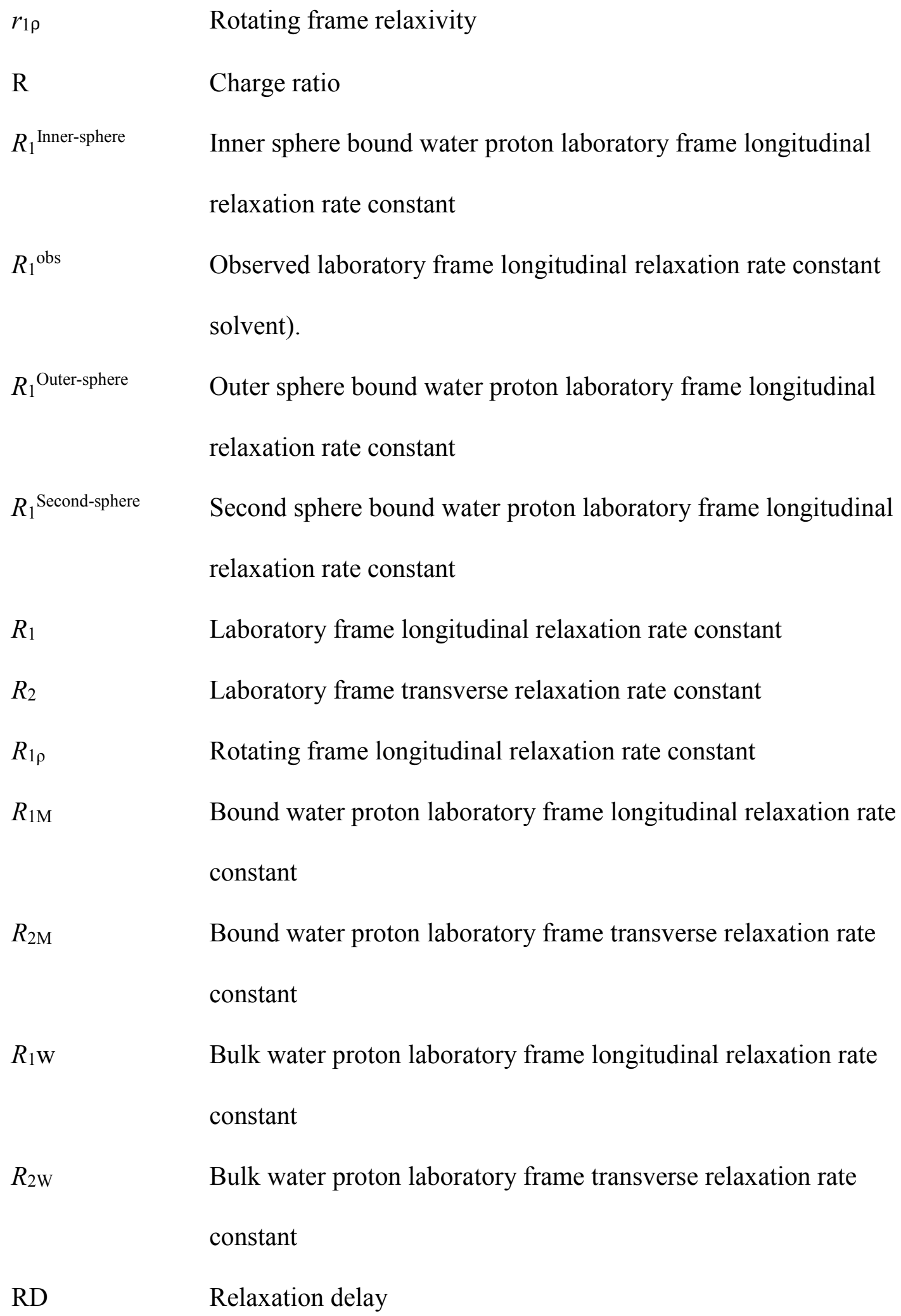




\begin{tabular}{|c|c|}
\hline RF & Radiofrequency \\
\hline SBM & Solomon-Bloembergen-Morgan \\
\hline $\mathrm{SE}$ & Spin echo \\
\hline $\mathrm{SD}$ & Standard deviation \\
\hline SEM & Scanning electron microscopy \\
\hline SNR & Signal to noise ratio \\
\hline$T_{1}$ & Laboratory frame longitudinal relaxation time constant \\
\hline$T_{2}$ & Laboratory frame transverse relaxation time constant \\
\hline$T_{1 \rho}$ & Rotating frame longitudinal relaxation time constant \\
\hline$T_{1 \mathrm{M}}$ & Bound water proton longitudinal relaxation time constant \\
\hline TL & Locking time \\
\hline TTHA & $\begin{array}{l}\text { 3,6,9,12-tetrakis(carboxymethyl)-3,6,9,12-tetraazatetradecanedioic } \\
\text { acetate }\end{array}$ \\
\hline$\tau$ & Delay time \\
\hline$\tau_{\mathrm{c} 1}$ & Longitudinal correlation time constant \\
\hline$\tau_{\mathrm{c} 2}$ & Transverse correlation time constant \\
\hline$\tau_{\mathrm{M}}$ & Inner-sphere water residence time constant \\
\hline$\tau_{\mathrm{R}}$ & Rotational correlation time constant \\
\hline$\tau_{\mathrm{S} 1}$ & Longitudinal electron relaxation time constant \\
\hline$\tau_{\mathrm{S} 2}$ & Transverse electron relaxation time constant \\
\hline$\tau_{\mathrm{v}}$ & Correlation time of the ZFS \\
\hline
\end{tabular}


US

VDU

Voxel

ZFS
Ultrasound

Visual display unit

3D pixel

Zero-field splitting 


\section{CHAPTER 1. INTRODUCTION}

\subsection{Magnetic resonance imaging: a critical imaging mode of the past, present and future}

\subsubsection{The rise to popularity of MRI}

In 1938 Isidor Rabi conducted the first nuclear magnetic resonance (NMR) experiments on Lithium chloride in his laboratory at Columbia University. ${ }^{1}$ In 1946 , and within a month of each other, teams at Harvard, lead by Purcell, ${ }^{2}$ and Stanford, lead by Bloch, ${ }^{3}$ had refined the technique and independently made observations of weak magnetism of protons in condensed matter. Awarded the Nobel Prize in physics for their work these were the founding fathers of NMR, which has since found many applications. One such application is magnetic resonance imaging (MRI). In 1973 Paul Lauterbur demonstrated

the imaging potential of MR by differentiating heavy and normal water in-vitro. ${ }^{4}$ The utility of such a technique was quickly recognized and the push towards the ultimate goal of imaging a living human began. In the late 1970's, shortly after Lauterbur's discovery, in-vivo images of the human finger ${ }^{5}$ and whole body imaging ${ }^{6}$ were published. Since, many technological advances including: cryogenic magnets, Gadolinium contrast agents, actively shielded magnets and the emergence of stronger magnetic fields for routine imaging to name just a few, have led to the current state of development of clinical MRI. ${ }^{7}$ 


\subsubsection{Modern day MRI and its comparison with other imaging modalities}

During this time, MRI has steadily become established amongst other imaging modalities available to radiologists including: X-ray, computed tomography (CT), ultrasound (US) and positron emission tomography (PET). Since the first commercial system in 1983 $\sim 20,000$ MRI systems have been installed worldwide ${ }^{7}$ with $\sim 27$ million scans (half the number of X-ray scans) being performed in the US alone in $2007 .{ }^{8}$ The popularity of MRI can largely be attributed to the technique being relatively non-invasive, employing non-ionizing radiation and having the ability to measure whole tissue samples with exceptional soft tissue spatial resolution and contrast, albeit at the expense of imaging time. However, the real power of MRI is in its flexibility. MRI has the ability to characterize several tissue parameters that can vary from tissue to tissue, allowing it to distinguish between healthy and diseased tissue and identify key markers of many diseases and disorders including those most prevalent in human kind. ${ }^{9-13}$ Moreover, this can be achieved whilst keeping the hardware required relatively constant and presenting clinically relevant information as easy-to-interpret 3D digital contrast images based on signal intensity. The signal in an MR image is typically generated from the water proton nuclei, the most abundant nuclei in vivo, maximizing the technique's sensitivity. Despite this, the sensitivity of MRI remains poor in comparison to X-ray-based methods, PET and US. However, MRI offers the advantage of fewer safety concerns. Although US is also considered very safe and the sound waves employed afford excellent temporal resolution, its drawback comes from poor spatial resolution. As can be imagined, for 
accurate diagnosis and optimal treatment of disease a combination of these complimentary imaging modalities is often required.

\subsubsection{The future of clinical MRI}

The major drawback for MRI is the technique's lack of sensitivity which is related to the field strength $(B)$ of the magnet, measured in flux density (tesla, T). Since the introduction of NMR in the 1960's, and later MRI, the operating magnetic field strength for both has increased linearly over time, leading to small incremental gains in

sensitivity. ${ }^{14}$ The vast majority of clinical scanners today operate at 1.5 and $3 \mathrm{~T}$, however, many $4.7 \mathrm{~T}$ scanners are in preclinical use and human imaging is permitted up to $8 \mathrm{~T}^{7}$ There is no evidence to suggest that the current trend is about to change and as such larger magnets will dot the landscape in the not so distant future. At these high fields, scientists seek to further lower detection limits and increase spatial resolution as a result of the improved signal-to-noise ratio (SNR). ${ }^{15}$ For as long as there is both a clinical and commercial requirement for larger magnets, any technological challenge imposed shall be met. Such challenges to large magnets include, but are not limited to: instrument siting within current radiology departments, $B_{0}$ field homogeneity across large bore instruments and economics of the setup and running costs.

\subsection{Nuclear magnetic resonance (NMR) theory}

Although much of what follows is in the context of MR imaging, the theories are entirely grounded in those of NMR. Thus, it is important to be reminded of the underlying 
physics, the origin of the signal as it pertains to MRI and its detection for contrast generation.

\subsubsection{The Zeeman effect}

Atoms comprise of electrons and a positively charged nucleus containing protons and neutrons. Interactions between the nuclei of many isotopes and the magnetic fields that surround them, allows NMR to provide detailed molecular information. Fundamental to NMR is spin, a physical property of all nuclei. Spin is a purely quantum mechanical concept that can be represented by the classical vector model of angular momentum. However, unlike classical angular momentum, induced by rotation, spin angular momentum is an intrinsic property. The total spin angular momentum, $\boldsymbol{I}$, a vector quantity, is given by Equation 1.1 where $\hbar$ is the reduced Planck's constant (Planck's constant divided by $2 \pi$ ) and $I$ is the specific spin quantum number of the nuclei of interest, a positive multiple of $1 / 2$.

$$
\boldsymbol{I}=\hbar[I(I+1)]^{\frac{1}{2}}
$$

Isotopes with even mass numbers and even numbers of both protons and neutrons (e.g. ${ }^{12} \mathrm{C}_{6}$ ) posses a spin quantum number $(I)$ of zero and therefore no angular momentum and are NMR inactive. However, if the proton and neutron numbers are both odd (e.g. ${ }^{2} \mathrm{H}_{1}$ and ${ }^{14} \mathrm{~N}_{7}$ ) $I$ takes non-zero integer values. Isotopes with odd mass numbers and either

odd or even numbers of protons and neutrons, such as the main isotope of hydrogen $\left({ }^{1} \mathrm{H}_{1}\right)$ 
and the minor stable isotope of carbon $\left({ }^{13} \mathrm{C}_{6}\right)$, posses half-integer spin values. Isotopes with non-zero spin values posses angular momentum and are NMR active.

With respect to an arbitrarily chosen axis, shown projected with respect to the z-axis in Equation 1.2, the spin vector of the angular momentum can have several possible directions, a result of the quantized nature of the spin projection magnetic quantum number, $\mathrm{m}_{\mathrm{I}}$, for which quantum mechanics dictates there are $2 I+1$ possible values which range from $-I$ to $+I$ with integer steps.

$$
I_{z}=m_{I} \hbar
$$

The angular momentum of the nuclei gives rise to a nuclear magnetic moment $(\mu)$ proportional to the total spin angular momentum $(I)$ and the gyromagnetic ratio $(\gamma)$, a constant specific to the nuclei of interest (Equation 1.3).

$$
\mu=\gamma \boldsymbol{I}
$$

The magnetic moment of the proton creates a weak magnetic field that interacts with local magnetic fields. In MRI, the nucleus of interest is that of the abundant water proton $\left({ }^{1} \mathrm{H}_{1}\right)$ which has a spin quantum number $(I)$ of a $1 / 2$ and therefore two possible spin projection quantum numbers $\left(\mathrm{m}_{\mathrm{I}}\right)$ of $-1 / 2$ and $+1 / 2$. In the absence of an external magnetic field these two spin states have equal probability and therefore equal spin populations and are considered energetically degenerate. As a consequence of this degeneracy the total magnetic moment is zero. When a subject is immersed in the strong homogenous magnetic field $\left(B_{0}\right)$ of an MRI instrument two interesting processes occur simultaneously. 
One, the degeneracy of the two spin states is broken and the proton nuclei can either align parallel to $B_{0}$, denoted as $+1 / 2$ (low energy, $\alpha$ ), or anti parallel to $B_{0}$, denoted $-1 / 2$ (high energy, $\beta$ ). This is known as the Zeeman effect, where the energy is the product of the magnetic moment and $B_{0}$ (Equation 1.4).

$$
E=-\mu B_{0}
$$

For a magnetic field along the z-axis and from Equation 1.2 and 1.3, this can be stated as;

$$
E=-\mu_{z} B_{0}=-m_{I} \hbar \gamma B_{0}
$$

Where the difference in energy between the equally spaced spin states is;

$$
\Delta E=\hbar \gamma B_{0}
$$

This corresponds to a frequency $\left(v_{0}\right)$ of electromagnetic radiation that can be found as follows;

$$
\text { Given; } \begin{aligned}
\Delta E & =h v_{0}=\hbar \gamma B_{0} \\
v_{0} & =\frac{\gamma B_{0}}{2 \pi}
\end{aligned}
$$

For magnetic field strengths typical in MRI and NMR, these frequencies correspond to the RF region of the electromagnetic spectrum.

The extent to which each state is populated is governed by the Boltzmann distribution law (Equation 1.8) where $\mathrm{N}_{\alpha}$ and $\mathrm{N}_{\beta}$ are the number of protons in the low (anti parallel) 
and high (parallel) energy states respectively, $\mathrm{k}_{\mathrm{B}}$ is the Boltzmann distribution constant, $T$ is the absolute temperature and $\Delta \mathrm{E}$ is the difference in energy between the two states.

$$
\frac{N_{\alpha}}{N_{\beta}}=e^{\frac{\Delta E}{k_{B} T}}=e^{\frac{\hbar \gamma B_{0}}{k_{B} T}}
$$

Due to energy considerations a slight excess of spins reside in the lower energy spin state. From Equation 1.8 it can be seen that the spin populations are influenced by the strength of $B_{0}$ and temperature. For a typical magnetic field strength used in clinical MRI of 1.41 $\mathrm{T}$ and at a temperature of $37^{\circ} \mathrm{C}$, this population difference is approximately 1 spin per 100000 spins, i.e. $\sim 10 \mathrm{ppm}$.

Two, $B_{0}$ exerts torque on the individual magnetic moments of the proton nuclei and they begin to precess out of phase with the angular momentum, $I$, at some constant angle around the axis of $B_{0}$. The angular frequency of this precession, which corresponds to the energy difference between the two spin states as described above, is termed the Larmor frequency $\left(\omega_{0}\right)$ and is dependent on $\gamma$ and the strength of $B_{0}$ (Equation 1.9). Equation 1.9 describes the Larmor frequency for a proton with respect to the direction of precession about $B_{0}$, where the negative sign indicates a negative sense of precession (clockwise) when viewed from a position above the direction of $B_{0}$.

$$
\omega_{0}=-\gamma B_{0}
$$


The end result of these two processes is that a net macroscopic magnetization (M), a vector sum of all the individual spin magnetic moments, is induced. When viewed from the rotating frame of reference (i.e. the $\mathrm{x}, \mathrm{y}$-axis is rotating at the Larmor frequency), $\mathrm{M}$ lies directly along the z-axis, longitudinal to the direction of $B_{0}$. Figure 1.1 summarizes the influence of $B_{0}$ on a collection of proton nuclei.

A

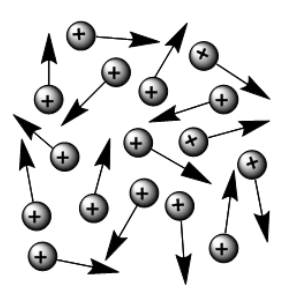

B

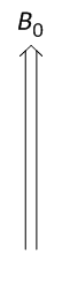

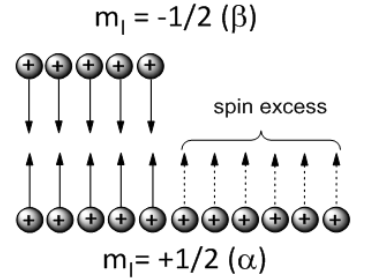
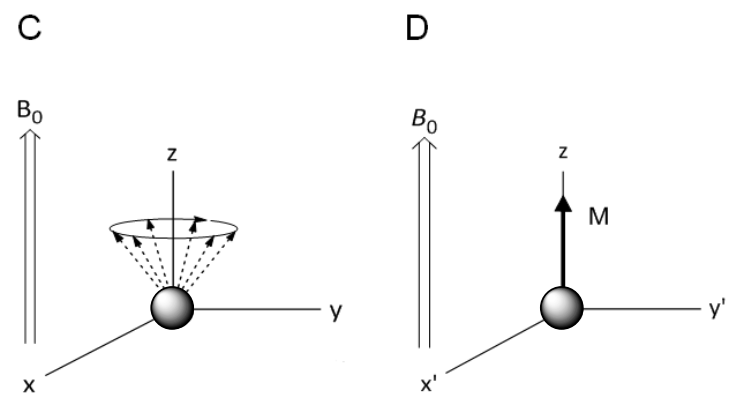

Figure 1.1: Summary of the influence of $B_{0}$ on a collection of proton nuclei. a) Degenerate nuclear spin orientations in the absence of $B_{0}$. b) Non-degenerate nuclear spin orientations in the presence of $B_{0}$. c) Excess spins randomly precessing around $B_{0}$. d) Net magnetization longitudinal to the direction of $B_{0}$ viewed from the rotating frame of reference.

\subsubsection{Signal detection}

In the above section a net magnetization vector (M) was established. This represents a vector sum of the entire ensemble of spins, whose magnitude is determined by the population difference (spin excess) between spin states. This difference in population, on the order of parts per million (ppm), is the origin of the MRI signal. But, it is also the source of the relatively poor sensitivity of MRI. Unfortunately the nuclear longitudinal magnetization is practically undetectable as its signal is swamped by three orders of magnitude of diamagnetism derived from the electrons (diamagnetic shielding). ${ }^{16}$ 
In order to detect the net magnetization it must be rotated in to the $\mathrm{x}, \mathrm{y}-\mathrm{plane}$, transverse to the direction of $B_{0}$. This is achieved by perturbation of the magnetization with a second magnetic field $\left(B_{1}\right)$ that is weak, relative to $B_{0}$, and generated by the application of a RF pulse, typically along the $\mathrm{x}$-axis. The result is that the magnetization is effectively rotated away from $B_{0}$ and the diamagnetism of electrons where it can be detected. In order for this to occur, the frequency of $B_{1}$ must match the Larmor frequency of the magnetic moments causing the spins to resonate. Under these conditions, the spins in the lower energy state absorb the RF energy and are promoted to the higher energy state, resulting in equalization of the spin populations. In addition, the $B_{1}$ pulse causes the individual magnetic moments to begin to precess together, i.e. in phase (phase coherent), and for magnetization to grow in the $\mathrm{x}, \mathrm{y}$-plane (transverse magnetization). The extent of the rotation of the magnetization is dependent on the strength and duration of the $B_{1}$ field, with maximum signal intensity being observed when the magnetization is rotated $90^{\circ}$. The net magnetization precesses at the Larmor frequency $\left(\omega_{0}\right)$ in the $\mathrm{x}, \mathrm{y}$-plane, generating a rotating magnetic field and an associated electric field. This electric field drives an oscillating electric current (the signal) in a wire coil near the sample in the $\mathrm{x}, \mathrm{y}-$ plane. Figure 1.2 summarizes the influence of a $90^{\circ} B_{1}$ pulse on the net magnetization.

\subsubsection{Longitudinal relaxation}

After the pulse is turned off the gate to the RF receiver opens and a free induction decay (FID) signal is detected and the spins relax back to thermal equilibrium. The thermal equilibrium state is that in which the spin populations of the energy levels are those 
predicted by the Boltzmann distribution and one in which no coherences exist, i.e. no component of the net magnetization is in the $\mathrm{x}, \mathrm{y}-\mathrm{plane}$. The return of the spin populations back to those predicted by the Boltzmann distribution, where an excess of spins are in the low energy spin state, requires dissipation of energy from the high energy spin states. This occurs as a result of field fluctuations that arise primarily from magnetic dipole-dipole interactions. This regenerates magnetization longitudinal to $B_{0}$ along the $\mathrm{z}$ axis and is termed longitudinal relaxation. Longitudinal relaxation is characterized by the time constant $T_{1}$, and the rate of the change of magnetization follows first order kinetics.
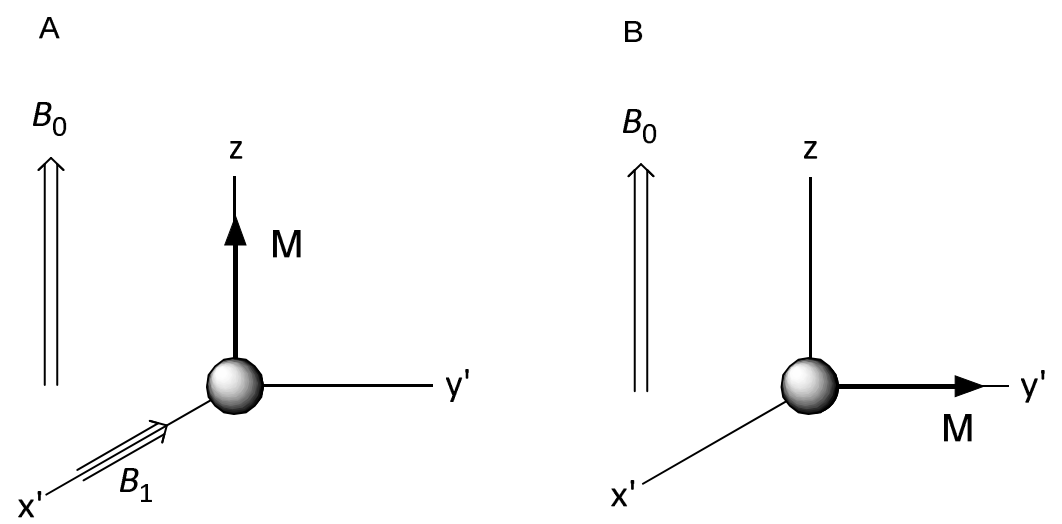

Figure 1.2: A summary of the influence of a $90^{\circ} \mathrm{RF} B_{1}$ pulse on the net magnetization. a) $A 90^{\circ}$ RF pulse promotes spins from the low energy state to the high energy state. b) When the RF pulse is complete the spin populations are equalized and precess in phase. The net magnetization has rotated into the $\mathrm{x}, \mathrm{y}$-plane generating transverse magnetization.

Derivation of the general solution to the integrated first order rate law demonstrates that the return of the magnetization to equilibrium from its position immediately after a RF pulse is turned off, is exponential in form, Equation 1.10, where $\mathrm{M}_{\mathrm{z}}(\tau)$ is the magnetization at a given delay time $(\tau), \mathrm{M}_{\mathrm{z}(\mathrm{eq})}$ is the equilibrium magnetization after 
complete recovery, $\mathrm{M}_{\mathrm{z}}(0)$ is the magnetization at the time, $\tau=0$, directly after a RF pulse, $T_{1}$ is the longitudinal relaxation time constant and $e^{-\frac{\tau}{T_{1}}}$ is the exponential decay function.

$$
\begin{gathered}
\text { rate }=k\left(M_{z}(\tau)-M_{z(e q)}\right) \\
\text { Where the rate constant, } k=\frac{1}{T_{1}} \\
\text { rate }=-\frac{d M_{z}(\tau)}{d \tau}=\frac{1}{T_{1}}\left(M_{z}(\tau)-M_{z(e q)}\right) \\
\frac{d M_{z}(\tau)}{\left(M_{z}(\tau)-M_{z(e q)}\right)}=-\frac{1}{T_{1}} d \tau \\
\int \frac{d M_{z}(\tau)}{\left(M_{z}(\tau)-M_{z(e q)}\right)}=-\frac{1}{T_{1}} \int d \tau \\
\ln \left(M_{z}(\tau)-M_{z(e q)}\right)=-\frac{1}{T_{1}} \tau+C
\end{gathered}
$$

If, at time $\tau=0$, the magnetization is $M_{Z}(0)$, then the constant of integration, $\mathrm{C}$, is;

$$
\begin{gathered}
\ln \left(M_{z}(0)-M_{z(e q)}\right)=C \\
\therefore \ln \left(M_{z}(\tau)-M_{z(e q)}\right)=-\frac{\tau}{T_{1}}+\ln \left(M_{z}(0)-M_{z(e q)}\right) \\
\ln \left(M_{z}(\tau)-M_{z(e q)}\right)-\ln \left(M_{z}(0)-M_{z(e q)}\right)=-\frac{\tau}{T_{1}}
\end{gathered}
$$




$$
\begin{aligned}
& \ln \frac{\left(M_{z}(\tau)-M_{z(e q)}\right)}{\left(M_{z}(0)-M_{z(e q)}\right)}=-\frac{\tau}{T_{1}} \\
& e^{\ln \left(\frac{M_{Z}(\tau)-M_{Z(e q)}}{M_{Z}(0)-M_{Z(e q)}}\right)}=e^{-\frac{\tau}{T_{1}}} \\
& \frac{M_{z}(\tau)-M_{z(e q)}}{M_{z}(0)-M_{z(e q)}}=e^{-\frac{\tau}{T_{1}}} \\
& M_{z}(\tau)-M_{z(e q)}=\left(M_{z}(0)-M_{z(e q)}\right) e^{-\frac{\tau}{T_{1}}} \\
& M_{z}(\tau)=M_{z(e q)}+\left(M_{z}(0)-M_{z(e q)}\right) e^{-\frac{\tau}{T_{1}}} \\
& M_{z}(\tau)=M_{z(e q)}+(-1)\left(M_{z(e q)}-M_{z}(0)\right) e^{-\frac{\tau}{T_{1}}} \\
& M_{z}(\tau)=M_{z(e q)}-\left(M_{z(e q)}-M_{z}(0)\right) e^{-\frac{\tau}{T_{1}}}
\end{aligned}
$$

Exponential recovery of the $\mathrm{z}$ component of the net magnetization towards equilibrium along the $\mathrm{z}$ axis after a $90^{\circ} \mathrm{RF}$ pulse (Equation 1.11) can be derived by expressing Equation 1.10 as:

$$
M_{Z}(\tau)=M_{z(e q)}-M_{z(e q)}\left(1-\frac{M_{z}(0)}{M_{z(e q)}}\right) e^{-\frac{\tau}{T_{1}}}
$$

This simplifies to;

$$
M_{z}(\tau)=M_{z(e q)}\left(1-\left(1-\frac{M_{z}(0)}{M_{z(e q)}}\right) e^{-\frac{\tau}{T_{1}}}\right)
$$


For a $90^{\circ}$ pulse $\mathrm{M}_{\mathrm{z}}(0)$ is 0 , and if we let $\mathrm{M}_{\mathrm{z}(\mathrm{eq})}$ be 1 , then this simplifies further to;

$$
\begin{gathered}
M_{z}(\tau)=1\left(1-\left(1-\frac{0}{M_{z(e q)}}\right) e^{-\frac{\tau}{T_{1}}}\right) \\
M_{z}(\tau)=M_{z(e q)}\left(1-(1-0) e^{-\frac{\tau}{T_{1}}}\right) \\
M_{Z}(\tau)=M_{z(e q)}\left(1-(1) e^{-\frac{\tau}{T_{1}}}\right) \\
M_{z}(\tau)=M_{z(e q)}\left(1-e^{-\frac{\tau}{T_{1}}}\right)
\end{gathered}
$$

For a delay time $(\tau)$ equal to one time constant $\left(T_{1}\right)$ it can be shown that the magnetization recovers $63 \%$ towards equilibrium (1) on the $\mathrm{z}$ axis.

$$
M_{z}\left(\tau=T_{1}\right)=1\left(1-e^{-\frac{T_{1}}{T_{1}}}\right)=1\left(1-\frac{1}{e}\right)=1\left(1-\frac{1}{2.718}\right)=0.63=63 \%
$$

$T_{1}$ values are often determined using an inversion recovery pulse sequence. This pulse sequence employs an initial $180^{\circ} \mathrm{RF}$ pulse that inverts the net magnetization to the $-\mathrm{Z}$

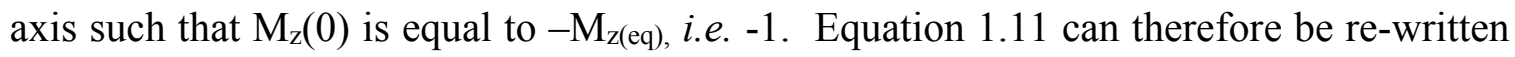
to describe the recovery of the net magnetization after a $180^{\circ} \mathrm{RF}$ pulse as;

$$
M_{z}(\tau)=1\left(1-2 e^{-\frac{\tau}{T_{1}}}\right)
$$


After one $T_{1}, 63 \%$ of the magnetization will recover towards equilibrium on the $+\mathrm{z}$ axis $(+1)$. Since the initial magnetization is on the $-\mathrm{Z}$ axis $(-1)$, the magnetization after one $T_{1}$ is $63 \%$ of 2 plus the initial magnetization $(-1)$, i.e. $2 \times(0.63)+-1=0.26$, as shown;

$$
M_{Z}\left(\tau=T_{1}\right)=1\left(1-2 e^{-\frac{T_{1}}{T_{1}}}\right)=1\left(1-2 \frac{1}{e}\right)=1\left(1-2 \frac{1}{2.718}\right)=0.26
$$

The inversion recovery pulse sequence consists of a series of radiofrequency (RF) pulses and time delays ${ }^{17}$ (Figure 1.3). An initial $180^{\circ} \mathrm{RF}$ pulse along the $+\mathrm{x}$ axis $\left(B_{1(180) \mathrm{x}}\right)$ (1) inverts the net magnetization from its equilibrium position along the $+\mathrm{z}$ axis parallel to $B_{0}$ to the $-\mathrm{z}$ axis (2). During the pulse delay $(\tau)$ the magnetization is allowed to relax back towards equilibrium, after which a $90^{\circ} \mathrm{RF}$ pulse is employed along the $+\mathrm{x}$ axis $\left(B_{1(90) \mathrm{x}}\right)$ (3) and converts the remaining magnetization into the $\mathrm{x}, \mathrm{y}$-plane where the FID signal is detected (4). A relaxation delay time (RD) allows full relaxation of the magnetization back to equilibrium to occur prior to the next iteration of the pulse sequence (1) with a longer pulse delay time $(\tau)$.

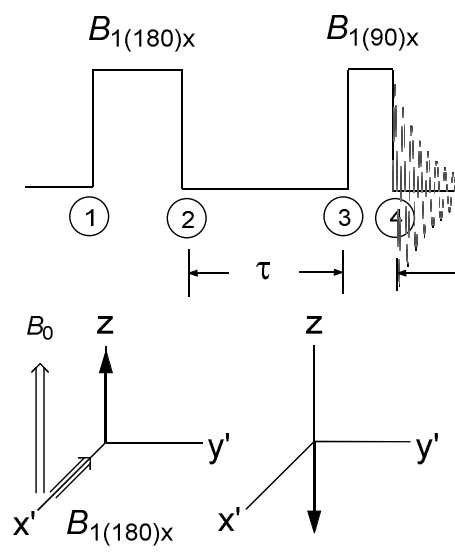

(1)
(2)

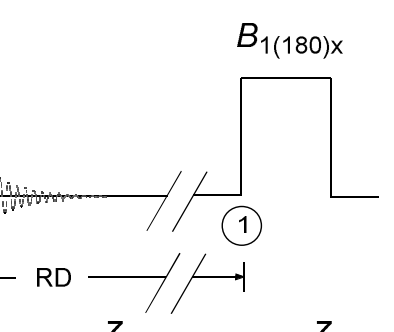

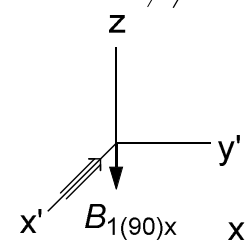

(3)

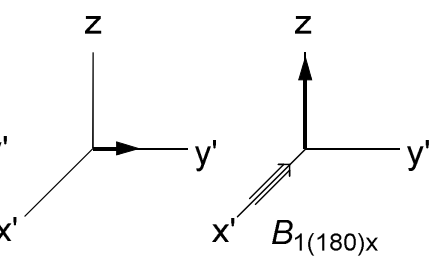

(4)
(1)

Figure 1.3: IR timing diagram (top), course and position of the net magnetization (bottom). 


\subsubsection{Transverse relaxation}

The loss of phase coherence of spins, transverse relaxation, occurs via fluctuating magnetic fields caused by molecular motions and $B_{0}$ field inhomogeneities. Transverse relaxation is characterized by the time constant $T_{2}$, the decay time constant for the loss of magnetization in the $\mathrm{x}, \mathrm{y}$-plane and the return to its equilibrium value (0), and also follows first order kinetics. Derivation of the solution to the integrated first order rate law demonstrates that the loss of transverse magnetization is also exponential in form, Equation $1.13, \mathrm{M}_{\mathrm{xy}}(\tau)$ is the magnetization at a given delay time $(\tau), \mathrm{M}_{\mathrm{xy}}(0)$ is the magnetization at time 0 directly after a RF pulse, $T_{2}$ is the transverse relaxation time constant and $e^{-\frac{\tau}{T_{2}}}$ is the exponential function of the decay.

$$
M_{x y}(\tau)=M_{x y}(0) e^{-\frac{\tau}{T_{2}}}
$$

After a $90^{\circ}$ pulse $\mathrm{M}_{\mathrm{xy}}(0)$ is 1 . From Equation 1.13 it can be determined that for a delay time $(\tau)$ equal to one time constant $\left(T_{2}\right)$, magnetization in the $\mathrm{x}, \mathrm{y}$-plane decays to $37 \%$ of its original value (1);

$$
M_{x y}\left(\tau=T_{2}\right)=1 e^{-\frac{T_{2}}{T_{2}}}=1\left(\frac{1}{e}\right)=1\left(\frac{1}{2.718}\right)=0.37=37 \%
$$

$T_{2}$ can be determined from a CPMG (Carr-Purcell-Meiboom-Gill) pulse sequence named after the contributing scientists responsible for its development. The CPMG pulse sequence consists of a $90^{\circ}$ excitation pulse followed by a $180^{\circ}(\pi)$ pulse that refocuses 
de-phasing spins in the $\mathrm{x}, \mathrm{y}$-plane to produce an echo signal. However, to ensure time constants more closely reflect the true $T_{2}$ value rather than $T_{2}{ }^{*}$ which includes contributions due to de-phasing that arises from the inhomogeneity of $B_{0}$, a train of phase encoded $180^{\circ}$ pulses are employed. The alternating phase of each $180^{\circ}$ pulse of the train reduces the inherent errors associated with each pulse through averaging, thereby improving the accuracy of $T_{2}$ values. $^{18}$ With respect to Figure 1.4, the CPMG pulse sequence employs a $90^{\circ} \mathrm{RF}$ pulse $\left(B_{1}\right)$ (1) that converts the net magnetization into the $\mathrm{x}, \mathrm{y}$-plane (2). The FID here is ignored. Instead, during a pulse delay time $(\tau)$ the spins begin to dephase, after which a $180^{\circ}$ pulse is applied (3). The $180^{\circ}$ pulse inverts the ensemble of spins (4) and they begin to re-phase such that after another pulse delay time $(\tau)$ the coherences are restored and an echo signal is detected for which the FID signal is recorded (5). This is repeated for a train of $180^{\circ}$ pulses of alternating phase along the $y$ axis where the exponential decrease of the echo signal intensity is due to the effective $T_{2}$. After the final echo of the train a relaxation delay time (RD) allows full relaxation back to equilibrium prior to the next iteration of the pulse sequence (1).
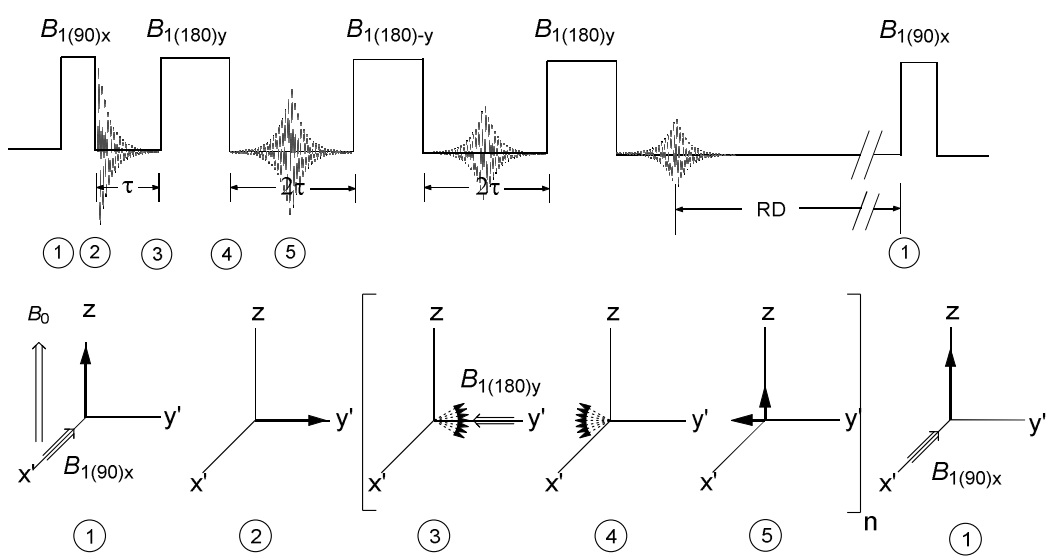

Figure 1.4: CPMG timing diagram (top), course and position of the net magnetization (bottom). 


\subsubsection{Fourier transformation, $T_{1}$ and $T_{2}$ determination}

The macroscopic transverse magnetization components of the FID in the $\mathrm{x}, \mathrm{y}-\mathrm{plane}$ as a function of time $t$, take the following forms;

$$
\begin{gathered}
M_{x}=\left(M_{e q}-M_{z}(t)\right) \sin (\omega t) e^{-\frac{t}{T_{2}}} \\
M_{y}=-\left(M_{e q}-M_{z}(t)\right) \cos (\omega t) e^{-\frac{t}{T_{2}}}
\end{gathered}
$$

The analogue FID signal picked up by a receiver coil in the $\mathrm{x}, \mathrm{y}$-plane is digitized for computer processing. Digitizing the FID produces a time domain function, $\mathrm{s}(\mathrm{t})$, where each time point corresponds to signal amplitude. The digitized signal is complex, i.e. comprises values from both the $\mathrm{x}$ and $\mathrm{y}$ components of the magnetization and is known as quadrature detection. The FIDs recorded for all iterations of the pulse sequence with varying pulse delay times $(\tau)$ are mathematically transformed to the frequency domain $S(\omega)$, a function of the initial signal amplitude in the time domain, $s(t)$. This is accomplished by a Fourier transformation (Equation 1.15) taking as the input both components of the net transverse magnetization, $\mathrm{M}_{\mathrm{x}}$ and $\mathrm{M}_{\mathrm{y}}$ from Equation 1.14.

$$
S(\omega)=\sum_{0}^{\infty} s(t)[\cos (\omega t)-i \sin (\omega t)]
$$

$T_{1}$ is determined by plotting the signal intensity ( $\mathrm{S}$ ) of the peak maximum in the frequency domain at (4) (Figure 1.5, top) as a function of $\tau$, the pulse delay time (Figure 1.5 , bottom) and fitting to Equation 1.12 

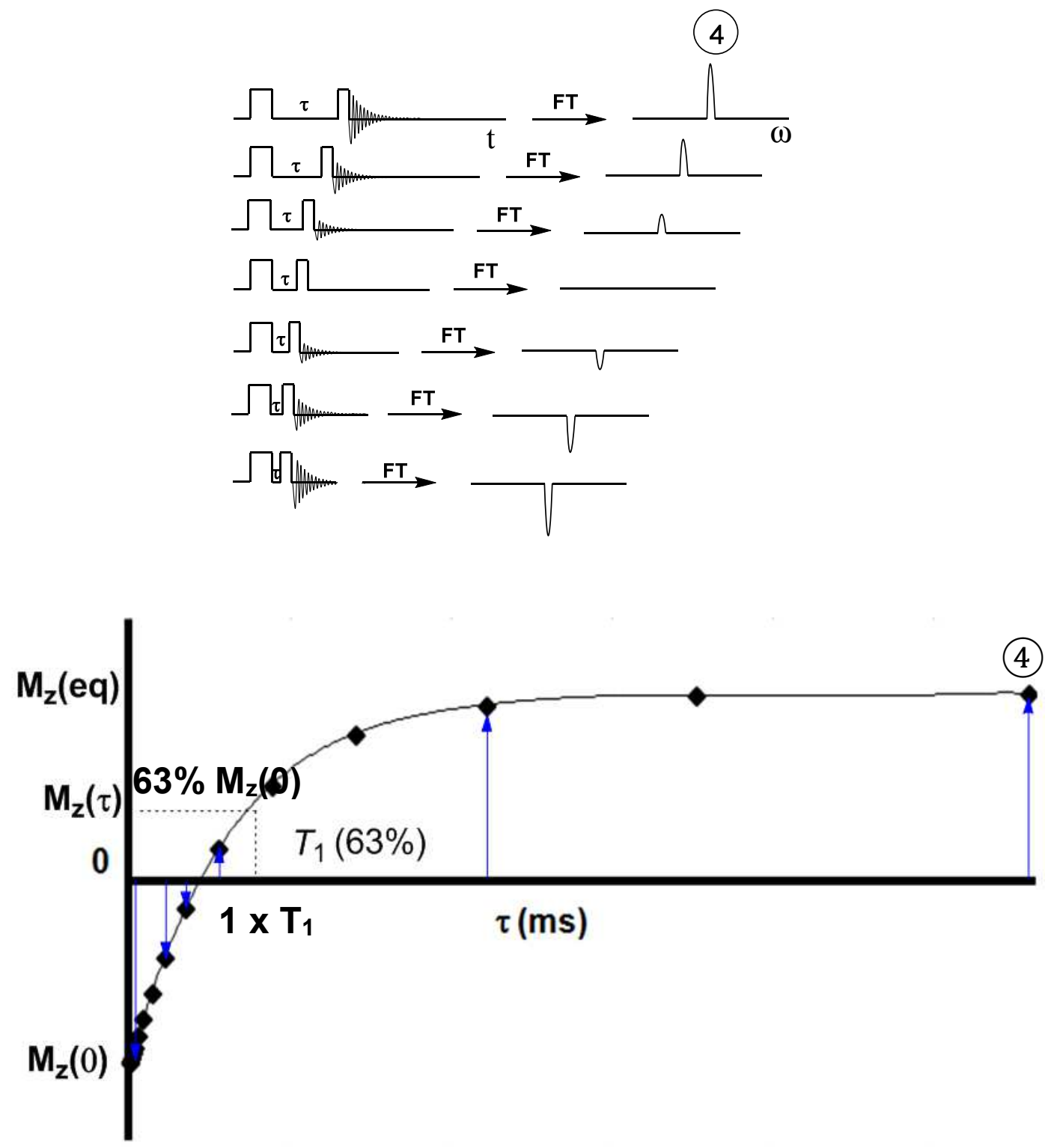

Figure 1.5: Summary of $T_{1}$ determination. IR pulse sequence and generation of spectra (top) and the evolution of magnetization $\left(\mathrm{M}_{\mathrm{z}}\right)$ as a function of the variable pulse delay time $(\tau)$ (bottom).

$T_{2}$ is determined by plotting the signal intensity (S) of the peak maximum in the frequency domain at (5) (Figure 1.6, top) as a function of $\tau$, the pulse delay time (Figure 1.6, bottom) and fitting to Equation 1.13. 

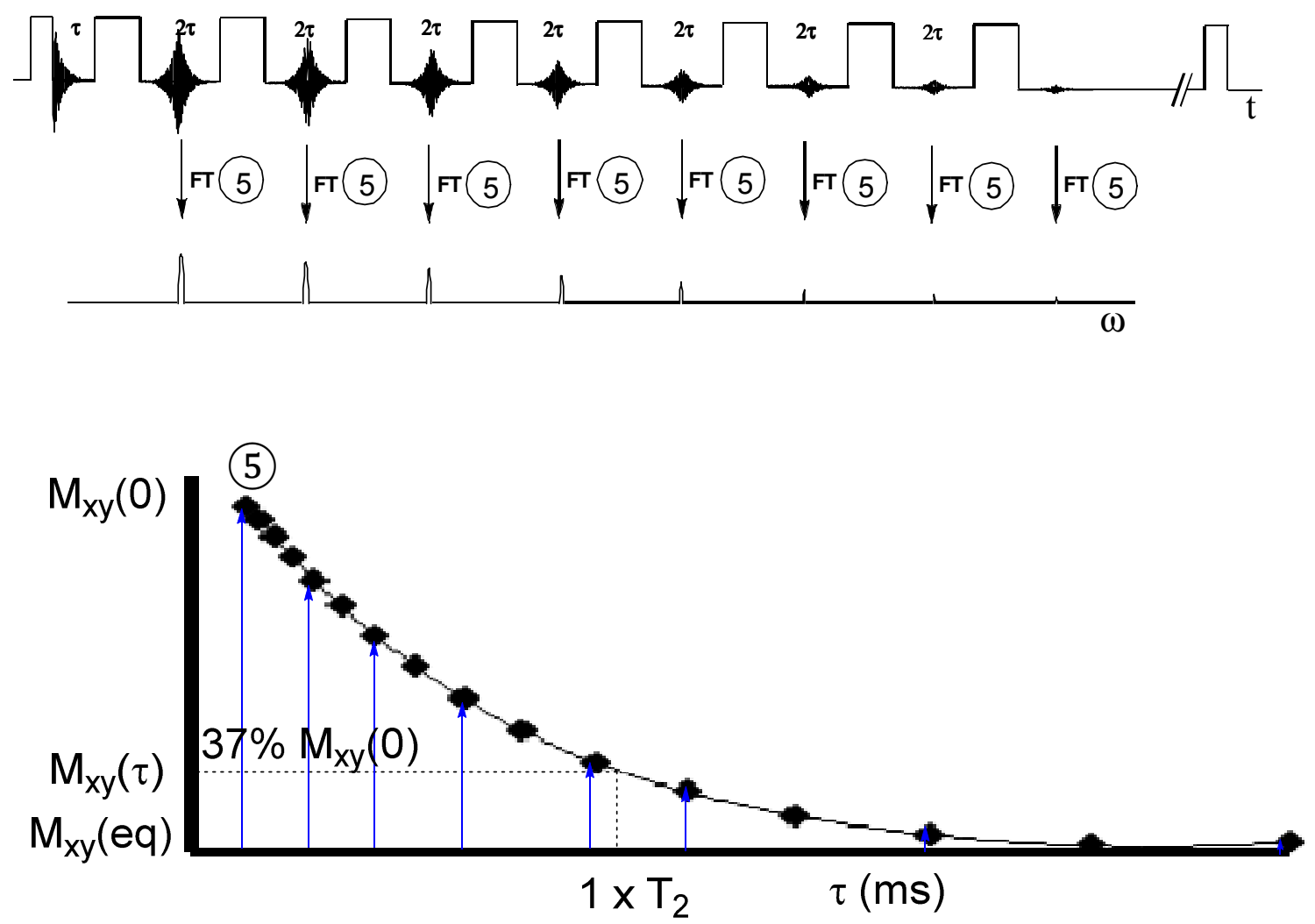

Figure 1.6: Summary of $T_{2}$ determination. CPMG pulse sequence and generation of spectra (top) and the decay of magnetization $\left(M_{x y}\right)$ as a function of the variable total pulse delay time $(\tau)$ (bottom).

\subsection{Inherent soft tissue contrast and image weighting}

The molecular composition of human soft tissue can be broadly divided into either water or macromolecules (lipids, carbohydrates, proteins etc). These molecules contain a vast number of hydrogen atoms (about $67 \%$ of all atoms in tissue), the source of signal in most MRI. By applying a gradient magnetic field on top of the static $B_{0}$ field, each hydrogen nuclei experiences a slightly different total magnetic field and resonate at slightly different Larmor frequencies. This introduces spatial encoding allowing a three- 
dimensional image to be generated. The signal intensity within an imaging voxel (1 $\mathrm{mm}^{3}$ ) is reported as relative brightness thus producing contrast in the image. The signal intensity is, in part, related to the number of hydrogen atoms in the voxel, proton density, a biologically variable parameter, and, to tissue $T_{1}$ and $T_{2}$ time constants discussed above, which also vary from tissue to tissue.

A spin echo (SE) pulse sequence ${ }^{19}$ is capable of extracting inherent proton density $\left[\mathrm{H}^{+}\right]$, $T_{1}$ or $T_{2}$ contrast. A traditional SE pulse sequence has the same features as a CPMG pulse sequence but with a single echo generated by a single $180^{\circ}$ refocusing pulse (Figure 1.7). With appropriate selection of the echo time (TE), the time between the initial $90^{\circ}$ RF pulse and the echo signal, and the repetition time (TR), the time between consecutive $90^{\circ} \mathrm{RF}$ pulses of subsequent scans, image contrast can be biased, or "weighted", towards any of the three contrast mechanisms discussed.

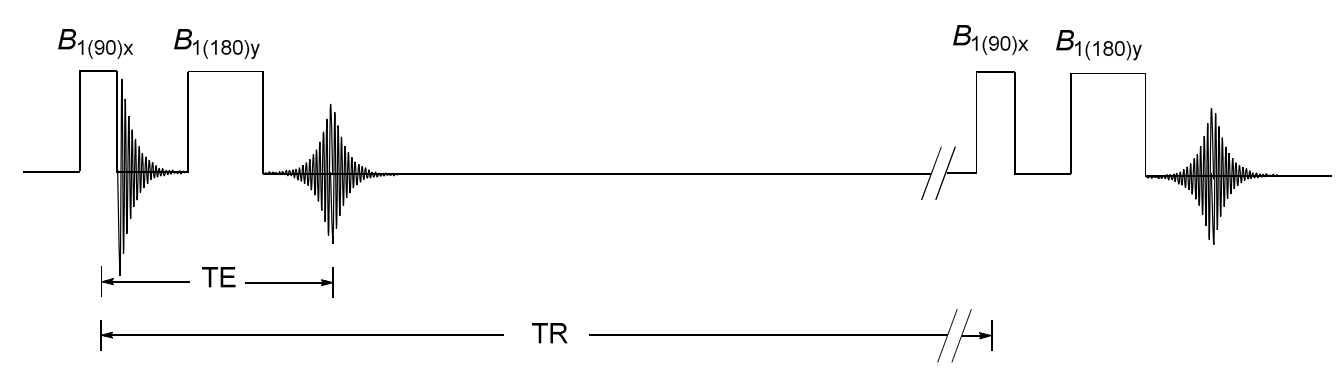

Figure 1.7: Spin echo pulse timing diagram for weighted imaging.

This can be seen from the signal intensity equation for this pulse sequence (Equation 1.16) where; $\mathrm{S}$ is the amplitude of the signal in the frequency domain, $\mathrm{k}$ is a constant that depends on the signal detection sensitivity of the instrument being used, $\left[\mathrm{H}^{+}\right]$is the 
proton concentration and $T_{1}$ and $T_{2}$ are the longitudinal and transverse relaxation time constants respectively. It is apparent from this equation that TR is related to $T_{1}$ and TE to $T_{2}$, and that $\left[\mathrm{H}^{+}\right]$is unrelated to either $T_{1}$ or $T_{2}$ but is always present to some extent.

$$
S=k\left[H^{+}\right]\left(1-e^{-\frac{T R}{T_{1}}} \cdot e^{-\frac{T E}{T_{2}}}\right)
$$

If TR is set to be longer than $T_{1}$ then the term $\mathrm{e}^{-\mathrm{TR} / T 1}$ tends to 0 and $T_{1}$ effects on image contrast will be suppressed. This is a result of negligible differences in signal intensity between two tissues with different $T_{1}$ values as magnetization recovers towards equilibrium. Likewise, if $\mathrm{TE}$ is set to be shorter than $T_{2}$ then the term $\mathrm{e}^{-\mathrm{TE} / T 2}$ tends to 1 and $T_{2}$ effects on image contrast will be suppressed. In this situation, little decay of the signal has occurred and again there is a negligible difference in signal intensity between two tissues with different $T_{2}$ values. If a SE pulse sequence is employed in which both $T_{1}$ and $T_{2}$ effects are suppressed (short TE and long TR) then image contrast will be weighted towards the remaining contribution, proton density, where greater numbers of protons in a voxel generates increased signal intensity. In practice, all images generated with the SE pulse sequence contain a mix of each contrast mechanism. Experimentation and experience is required to optimize the desired contrast between tissues of interest. A summary of the effects of different types of image weighting on brain tissue contrast between ventricles, that contains CSF (cerebrospinal fluid), an aqueous fluid, and white matter, that consists of a large amount of lipid containing myelin, is shown in Figure 1.8. $T_{1}$ and $T_{2}$ time constants for CSF and white matter were obtained from literature at 1.5 
$\mathrm{T}^{14}$, 20 Images were generated with the specified parameters using a web-based simulation $^{21}$ with consent from the creator.

CSF contains mostly water, a fast tumbling small molecule for which the frequency of the oscillations of the proton magnetic dipole is typically greater than the Larmor frequency. This results in inefficient dipole-dipole interactions due to short correlation times $\left(\tau_{c}\right)$, the time two dipoles spend interacting, and thus long time constants. Water molecules associated with macromolecules in soft tissue such as white matter and protons that are part of the macromolecular structure are less free to rotate. As such, the oscillation of the proton magnetic dipole is closer to the Larmor frequency leading to longer correlation times and shorter time constants. If TR is long, $>>T_{1}$, and TE is short, $<<T_{2}$, then the resultant image is proton density-weighted as both $T_{1}$ and $T_{2}$ effects are suppressed (Figure 1.8, top row). In this case the proton density-weighted image is essentially isointense with little contrast between the bright internal volume of the ventricles and white matter. This is a result of high proton densities in the two tissues, the ventricles, which contains CSF (cerebrospinal fluid), a water based fluid, and white matter which consists of large amounts lipid containing myelin. If TR is long, $>>T_{1}$, and TE is approximately the average of the expected $T_{2}$ values of the tissues for which contrast is desired, then the image is $T_{2}$-weighted, with $T_{1}$ effects being suppressed (Figure 1.8, middle row). In a $T_{2}$-weighted image the CSF in the ventricles is hyperintense as a result of its longer $T_{2}$ value compared to white matter, which appears hypointense. Conversely, If TE is appropriately selected in order to suppress $T_{2}$ contrast, 
typically $<T_{2}$, and TR is approximately the average of the expected $T_{1}$ values of the tissues for which contrast is desired, then the image is $T_{1}$-weighted (Figure 1.8, bottom row). In a $T_{1}$-weighted image the CSF in the ventricles is hypointense as a result of its longer $T_{1}$ value compared to white matter, which appears hyperintense.
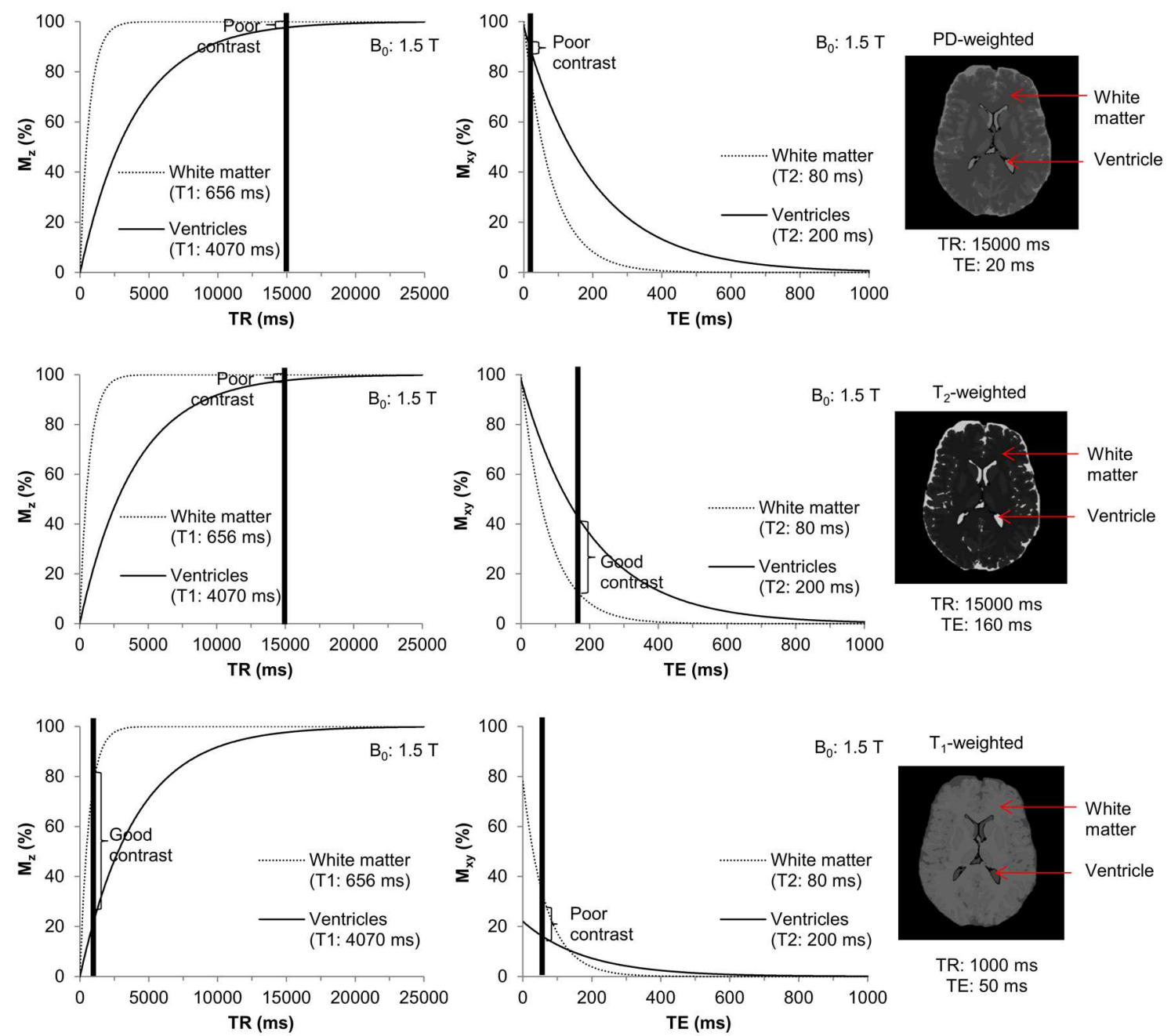

Figure 1.8: Effect of image weighting on contrast between CSF in the ventricles and white matter. Proton density-weighted (top), $T_{2}$-weighted (middle) and $T_{1}$-weighted (bottom). 


\section{4. $G d^{3+}$-based contrast agents for soft tissue contrast enhancement}

Contrast generated by $T_{1}$-weighting can be enhanced by the intravenous administration of an extracellular $\mathrm{Gd}^{3+}$-based contrast agent (CAs). In 2007, 43\% of the 27 million scans performed in the US required contrast enhancement from such agents. ${ }^{8}$ Their ability to increase contrast between tissue in which they are taken up (typically diseased) and

neighboring tissue (typically healthy) has been the subject of much research ${ }^{22-29}$ by chemists since the early 1980's when they were first introduced. ${ }^{30}$ This has led to unparalleled improvements in tissue discrimination by increasing water proton relaxation rate constants of tissue in which they are taken up relative to surrounding tissue that would otherwise be in a similar magnetic environment and generate little contrast. Contrast agents are particularly useful for the imaging of brain pathology. In healthy brain tissue an intact blood brain barrier (BBB) prevents a contrast agent from entering cells. However, in diseased tissue the BBB can become damaged allowing a contrast agent to enter. In this case, contrast agents can be thought of as a means of identify the disruption of the BBB, particularly useful for pathologies such as multiple sclerosis.

\subsubsection{Classification of clinically approved $G d^{3+}$-based $C A$}

Over the last 25 years or so several $\mathrm{Gd}^{3+}$-based CAs have been approved for clinical use in the US and EU and fall into one of three classes based on their biodistribution (Figure 1.9). All are extra cellular agents, with those that are excreted exclusively through the kidneys belonging to one class and those that exhibit some biliary clearance belonging to 
another. Those restricted to the intravascular compartment due to binding and their increased size make up a third class.

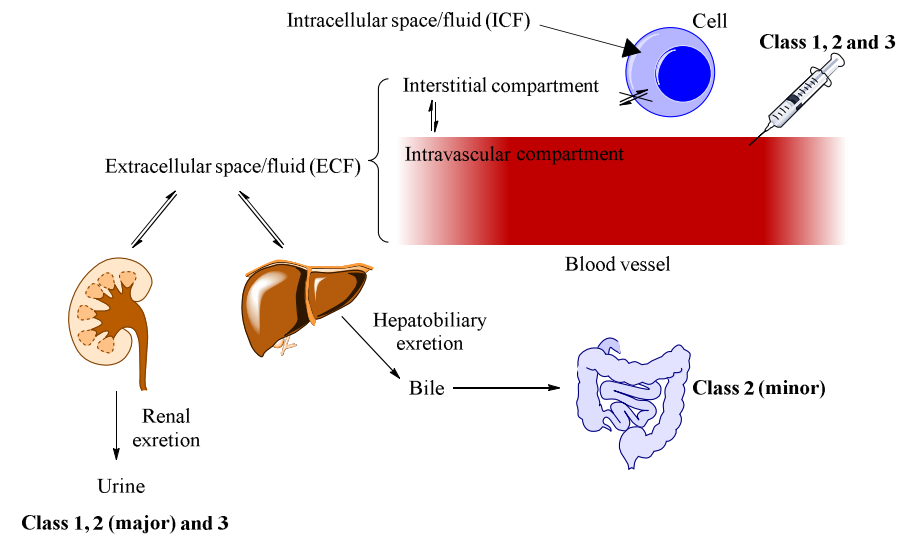

Figure 1.9: Distribution and excretion pathways for clinically approved $\mathrm{Gd}^{3+}$-based CAs.

Six of the nine FDA approved agents are very hydrophilic do not exhibit any specific binding properties (Figure 1.10). After intravenous injection they circulate in the extracellular fluid compartment (ECF) prior to elimination through the kidneys. Chelates of $\mathrm{Gd}^{3+}$ in which the diethylenetriaminepentacetate (DTPA) ligand scaffold is modified to contain a benzyl substituent (Figure 1.11) are taken up by hepatocytes ${ }^{31}$ and cleared, to a degree $(2-4 \%)$, via the hepatobiliary system making them useful for liver imaging application. The relaxivity of these agents are elevated compared to the ECF agents due to a slight binding function $(\sim 10 \%)$ between the benzyl group and plasma proteins. ${ }^{32}$ The third class of clinically approved $\mathrm{Gd}^{3+}$-based CAs contains just one agent, Ablavar, (Figure 1.12). Expanding on the success of MulitHance and Eovist/Primovist, Ablavar allows for an even greater increase in relaxivity to be observed via a reversible binding 
function with serum albumin that lengthens the rotational correlation time due to the large effective molecular weight. Ablavar contains a lipophilic biphenylcyclohexyl group for binding and restricts access to the interstitial compartment making it particularly useful for the imaging of the blood pool. The binding moiety is attached to a pendant arm of the DTPA ligand via a phosphodiester linker that results in Ablavar being eliminated renally.

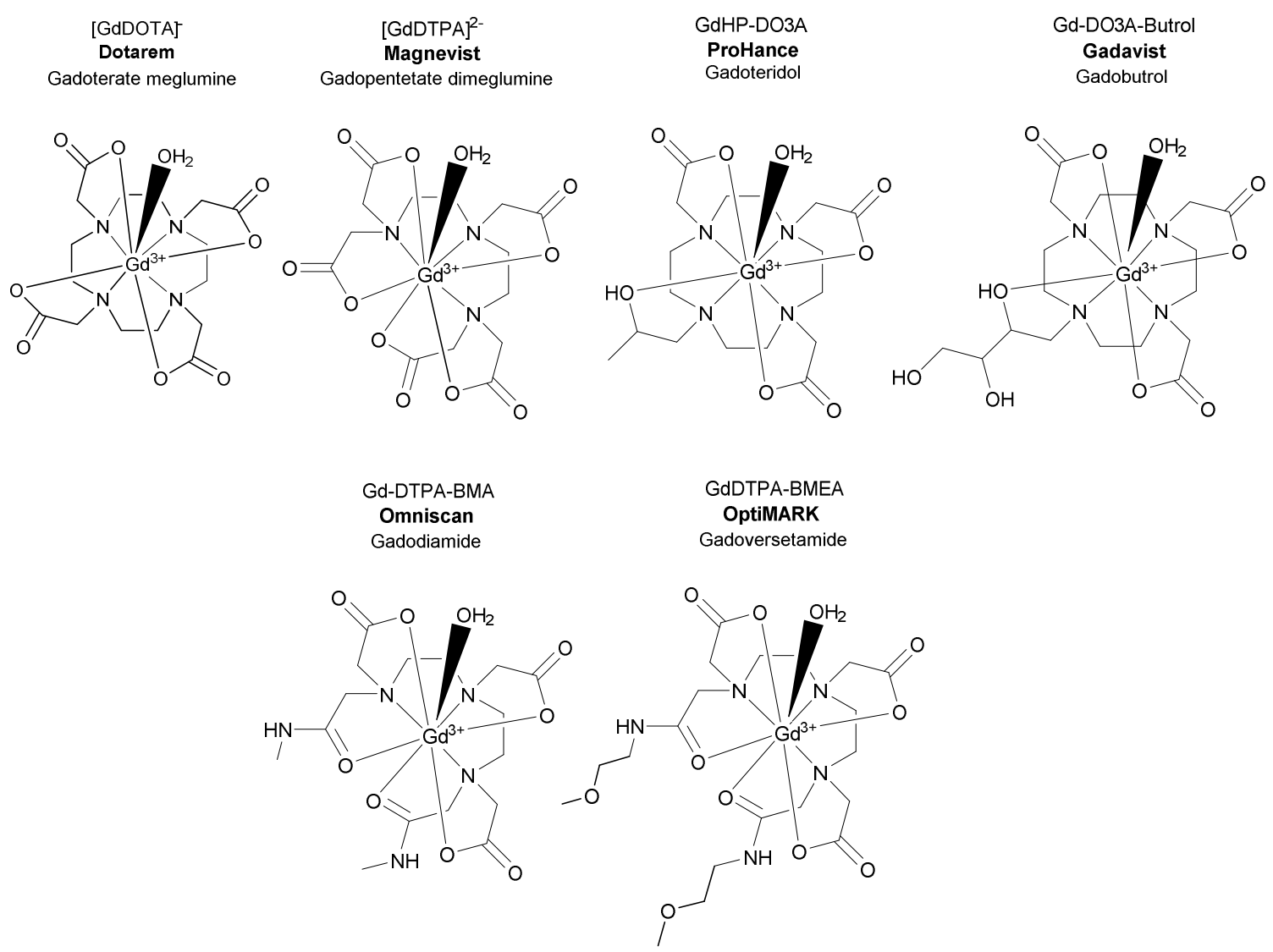

Figure 1.10: Class 1: Extracellular fluid (ECF) agents. 


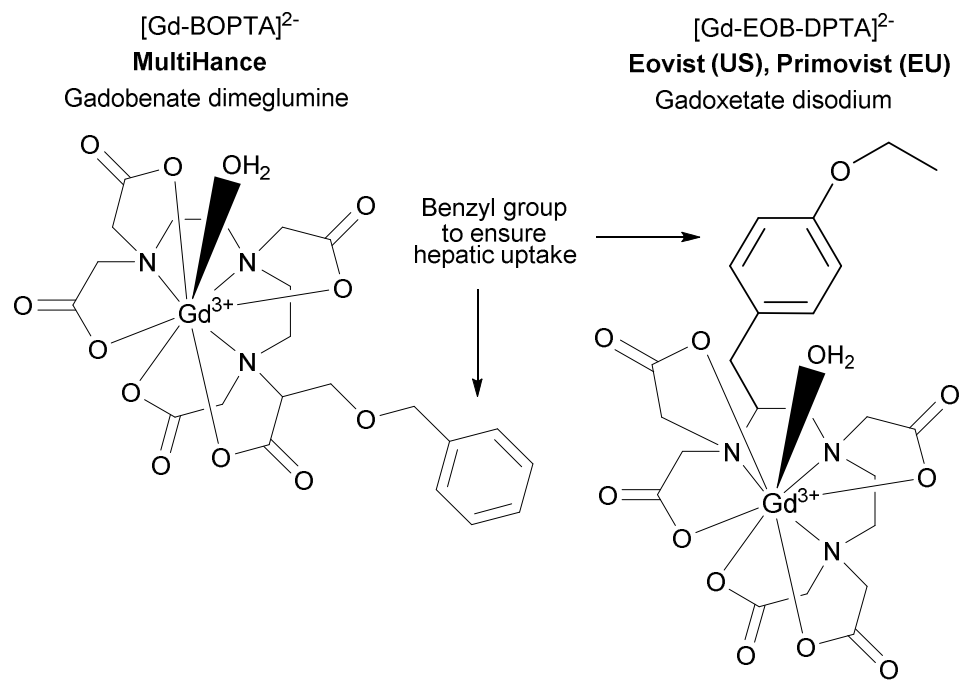

Figure 1.11: Class 2: Extracellular fluid (ECF) and liver agents.

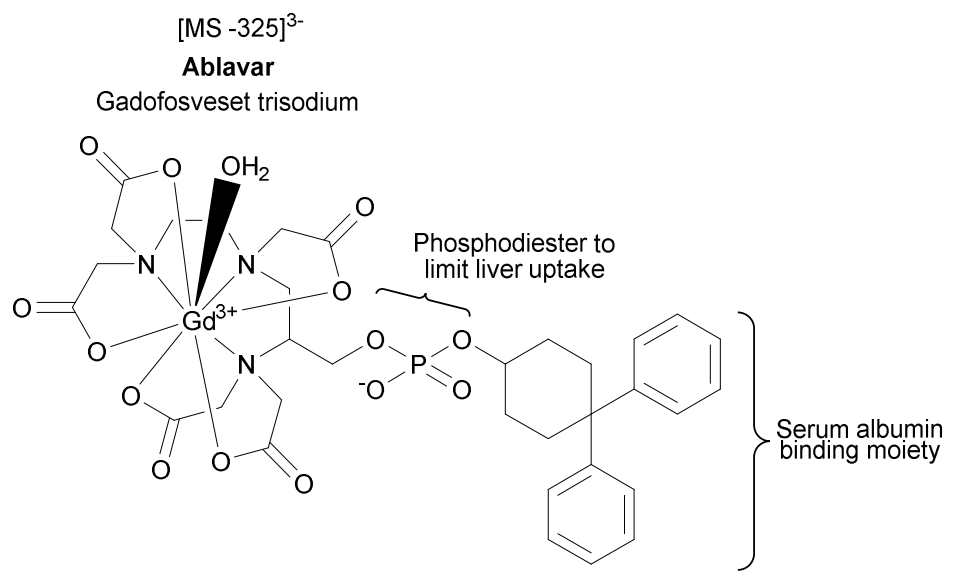

Figure 1.12: Class 3: Blood pool agent with serum albumin binding moiety.

These agents can be further categorized as being derived from macrocyclic (category A) or linear (category B) parent ligands (Figure 1.13) or bulky chelates (category C) (Table $1.1)$. 


\section{$\mathrm{H}_{4}$ DOTA}

2,2 ',2",2'"-(1,4,7,10-tetraazacyclododecane1,4,7,10-tetrayl)tetraacetic acid

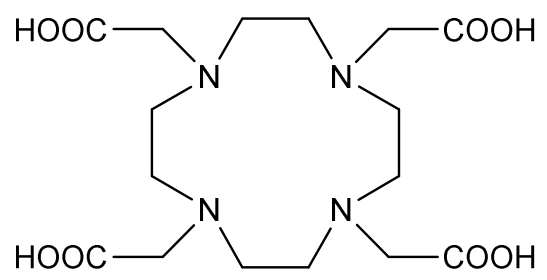

$\mathrm{H}_{5}$ DTPA

2,2',2",2"',2"'"-(1,2-ethanediylnitrilo)pentaacetic acid

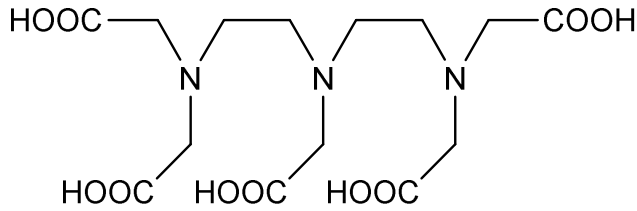

Figure 1.13: Macrocyclic and linear parent ligands for clinical $\mathrm{Gd}^{3+}$-based CAs.

Table 1.1: Physical properties of the three categories of $\mathrm{Gd}^{3+}$-based CAs studied.

\begin{tabular}{|c|c|c|c|c|c|c|}
\hline Trade name & $\begin{array}{c}\text { Plasma relaxivity } 1 . \\
r_{1}\end{array}$ & $\begin{array}{c}1.5 T 37^{\circ} \mathrm{C}\left(\mathrm{mM}^{-1} \mathrm{~s}^{-1}\right)^{\mathrm{a}} \\
r_{2}\end{array}$ & $\begin{array}{c}\text { Standard dose } \\
(\mathrm{mmol} / \mathrm{kg})\end{array}$ & $\begin{array}{c}\text { Formulation } \\
\text { concentration (M) }\end{array}$ & $\begin{array}{c}\text { Osmolality } \\
\left(\mathrm{Osm} / \mathrm{kg} \mathrm{H} \mathrm{H}_{2} \mathrm{O}\right)\end{array}$ & Formal charge \\
\hline \multicolumn{7}{|c|}{ Category A: Cyclic } \\
\hline Dotarem & 3.6 & 4.3 & 0.1 & 0.5 & 1.35 & $1-$ \\
\hline ProHance & 4.1 & 5.0 & 0.1 & 0.5 & 0.63 & 0 \\
\hline Gadovist & 5.2 & 6.1 & 0.1 & 1.0 & 1.60 & 0 \\
\hline \multicolumn{7}{|c|}{ Category B: Linear } \\
\hline Magnevist & 4.1 & 4.6 & 0.1 & 0.5 & 1.96 & $2-$ \\
\hline Omniscan & 4.3 & 5.2 & 0.1 & 0.5 & 0.79 & 0 \\
\hline \multicolumn{7}{|c|}{ Category C: Bulky } \\
\hline MultiHance & 6.3 & 8.7 & 0.1 & 0.5 & 1.97 & $2-$ \\
\hline Ablavar & $19^{b}$ & $34^{b}$ & 0.03 & 0.25 & 0.82 & 3- \\
\hline
\end{tabular}

(HSA) bound and free contributions. All other data readily obtainable from supplier information.

\subsubsection{Stabilization of $G d^{3+}$-based CAs}

Free $\mathrm{Gd}^{3+}$ is both insoluble at physiological $\mathrm{pH}$ and is known to be toxic. ${ }^{34}$ Toxicity arises due to the ability of $\mathrm{Gd}^{3+}$ to take part in transmetallation with other important biological cations such as $\mathrm{Ca}^{2+}, \mathrm{Cu}^{2+}$ and $\mathrm{Zn}^{2+}$, thus disrupting important cellular mechanisms, ${ }^{35}$ a factor worsened by its long residence time in the body and evidence 
showing deposits in skin and bone. ${ }^{36-41}$ Therefore, $\mathrm{Gd}^{3+}$-based CAs must be formulated with the metal ion bound extremely tightly to an organic ligand. This affords thermodynamically and kinetically stable nontoxic chelates which are cleared more rapidly than they dissociate, such as those in clinical use shown above in Figures $1.10-$ 1.12 .

\subsubsection{Mechanism of action of $G d^{3+}$-based CAs}

$\mathrm{Gd}^{3+}$-based CAs can be rationally designed for optimum per molar effectiveness (relaxivity) and for targeting specific tissue type to allow for more localized contrast improvement and improved clinical relevance and has been extensively published and reviewed $^{22-29}$ The basic pre-requisite of a CA for MRI is that it should increase the relaxation rate constant of bulk water protons. To that end, almost any paramagnetic solute could be used since relaxation processes occur as a consequence of the interactions between magnetic moments of unpaired electrons and those of interacting nuclei. However, the trivalent paramagnetic gadolinium ion $\left(\mathrm{Gd}^{3+}\right)$ has garnered most attention. This is due to its seven unpaired $f$ electrons (maximum available) and long electron relaxation times $\left(\tau_{S}\right)$ that allow for increased efficacy of relaxation of the interacting water proton nuclei. ${ }^{22}$ In the presence of a $\mathrm{Gd}^{3+}$ 'bound' water molecule, these properties lead to shorter longitudinal relaxation time constants for the bound water protons $\left(T_{1 \mathrm{M}}\right)$ which, when in fast exchange with bulk water, are able to transmit the paramagnetic relaxation enhancement (PRE) effect more effectively and thus increasing their relaxivity

$\left(r_{1}\right)$, described as the paramagnetic relaxation enhancement $\left(R_{1}^{\text {observed }}-R_{1}{ }^{\mathrm{w}}\right)$ normalized to 
the Gadolinium concentration (Equation 1.17). Where; $R_{1}{ }^{\text {observed }}$ is the observed tissue water proton longitudinal relaxation rate constant (the inverse time constant) and $R_{1}{ }^{\mathrm{w}}$ is the longitudinal relaxation rate constant of pure water (or other solvent).

$$
r_{1}=\frac{R_{1}^{\text {observed }}-R_{1}^{w}}{[G d]}
$$

\subsubsection{Contributions to the PRE effect}

The observed longitudinal relaxation rate constant $\left(R_{1}{ }^{\text {obs }}\right)$ of water protons in the presence of a $\mathrm{Gd}^{3+}$-based $\mathrm{CA}$ has several contributions as a consequence of various spheres of water molecules surrounding the metal ion, each of which are in a constant and rapid exchange with the bulk water protons and contribute to the paramagnetic relaxation enhancement to different extents (Equation 1.18). $R_{1}^{\text {Inner-sphere }}$ is the inner sphere relaxation rate constant, $R_{1}$ Second-sphere is the second sphere relaxation rate constant, $R_{1}$ Outersphere is the outer sphere relaxation rate constant and $R_{1}{ }^{\mathrm{w}}$ is the solvent relaxation rate constant. Figure 1.14 shows a cartoon representation of the several spheres of water surrounding a typical $\mathrm{Gd}^{3+}$-based CA.

$$
R_{1}^{\text {Observed }}=R_{1}^{\text {Inner-sphere }}+R_{1}^{\text {Second-sphere }}+R_{1}^{\text {Outer-sphere }}+R_{1}^{w}
$$

The coordination number of the $\mathrm{Gd}^{3+}$ ion ranges from eight to ten; however, it is typically nine as in the case of each of the chelates approved for clinical use. Eight are occupied by the organic chelating ligand and one by a single water molecule, the inner sphere water, which defines the hydration number $(q)$ of the complex. 


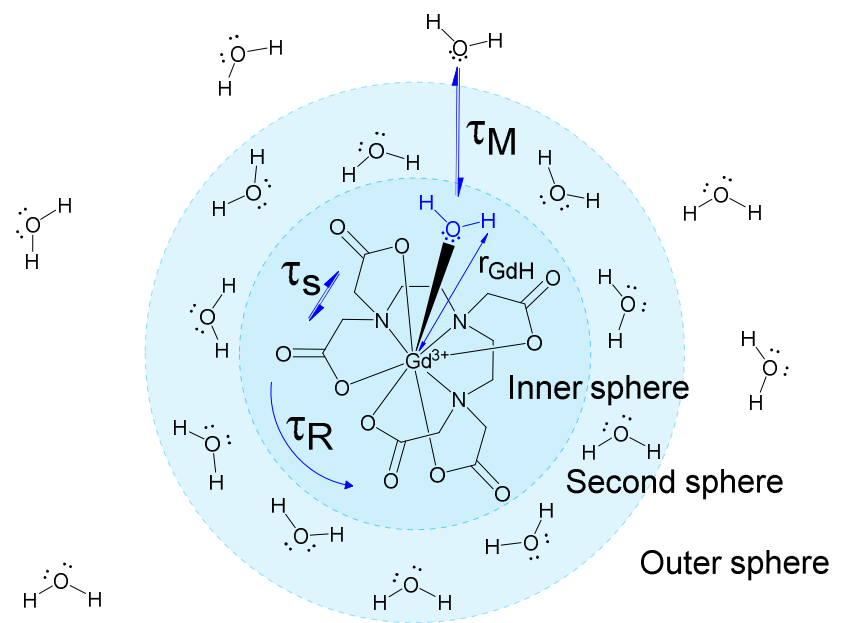

Figure 1.14: Schematic of the spheres of water that contribute to relaxivity of $\mathrm{Gd}^{3+}$-based CA.

A chelates inner sphere contribution $\left(R_{1}{ }^{\mathrm{IS}}\right)$ to $R_{1}{ }^{\text {obs }}$ is well described by SolomonBloembergen-Morgan (SBM) theory ${ }^{42-45}$ and has been shown to be dependent on the hydration number, the inner sphere water proton relaxation time constant $\left(T_{1 \mathrm{M}}\right)$, the water proton residence life time $\left(\tau_{\mathrm{M}}\right)$ and the molar concentration of $\mathrm{Gd}^{3+}(\mathrm{c})$ (Equation 1.19).

$$
R_{1}^{I S}=\frac{c \times q}{55.6}\left(\frac{1}{T_{1 M}^{H}+\tau_{M}}\right)
$$

Second sphere contributions $\left(R_{1}{ }^{\mathrm{SS}}\right)$ to $R_{1}{ }^{\text {obs }}$ occur due to an ordered sphere of water molecules beyond the inner sphere water molecules. Second sphere water molecules are in close proximity to the metal ion and are also well described by SBM theory and the parameters outline above for $R_{1}{ }^{\mathrm{IS}}$. Second sphere contributions have been shown to arise as a result of hydrogen bonding between the second sphere water molecules and lone pairs on the ligand groups. ${ }^{28}$ Outer sphere contributions $\left(R_{1}{ }^{\mathrm{OS}}\right)$ to $R_{1}{ }^{\text {obs }}$ occur due to 
diffusion of the complex through the water and can be described by modified Freeds equations. $^{46}$

\subsection{Traditional methods for optimization of relaxivity: A discussion of SBM theory}

\subsubsection{Motives for increasing relaxivity}

Relaxivity has long since been established by SBM theory to be magnetic field dependant. $^{42-45}$ This dependence can be determined using a field cycling relaxometer developed from the technical concepts of Anderson and Redfield. ${ }^{47}$ The instrument is able to rapidly measure relaxivity as a function of a large range of magnetic field strengths. A plot of such measurements is known as a nuclear magnetic relaxation dispersion (NMRD) profile. The NMRD profile for Dotarem, the original cyclic $\mathrm{Gd}^{3+}-$ based CA approved for clinical use, is shown in Figure 1.15 (dashed line). As can be seen the relaxivities are significantly reduced at current clinical imaging fields of $1.41-3$ $\mathrm{T}(60-128 \mathrm{MHz})$ and continue to decrease as field strength increases further, a considerable concern given the current trend towards greater magnetic field strengths ${ }^{14}$ that afford improved signal to noise. ${ }^{15}$

Since inherent tissue $T_{1}$ increases with increasing $B_{0},{ }^{48}$ agent detectability is improved. However, these gains are not fully realized due to the decrease in relaxivity at higher $B_{0}$ fields. This results in $\mathrm{Gd}^{3+}$-based CAs having high detection limits, a problem faced by chemists since their introduction into diagnostic radiology in the early $1980 \mathrm{~s},{ }^{49}$ and necessitates high doses be administered. High doses cause a significant reduction of the 
tissue $T_{1}$ and leads to an observable change in image contrast, determined from the change in $T_{1}$ in the presence of the agent with respect to the inherent $T_{1}$ of the tissue.

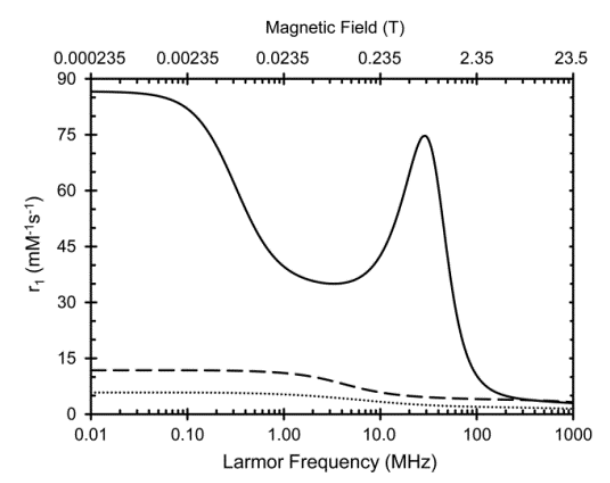

Figure 1.15: The nuclear magnetic relaxation dispersion (NMRD) profile of Dotarem (dashed line ${ }^{50}$ ) plotting laboratory frame relaxivity as a function of $B_{0}$ field, the outer-sphere contribution is shown by the dotted line. Also plotted is the NMRD profile of a theoretically "optimized" gadoterate chelate (solid line).

Despite of these significant shortcomings, tissue contrast in MRI is remarkable and spatial resolution unsurpassed. The driving force behind the quest for higher relaxivities comes from the desire to perform molecular imaging which entails selectively targeting CAs to biomarkers of disease pathology that exist in very low concentrations. Assuming 1:1 binding stoichiometry between target and agent it can be calculated that for current agents that have a relaxivity between 4 and $5 \mathrm{mM}^{-1} \mathrm{~s}^{-1}$, the target molecule must be present at $\sim 125 \mu \mathrm{M}$ or greater ${ }^{51}$, a value that limits the number of target candidates. Contrast agents with lower detection limits as a result of their high relaxivity will make this more feasible and shows the importance of optimizing agent relaxivities. 


\subsubsection{SBM parameters for optimizing relaxivity}

Researchers have given most attention to optimizing agent relaxivity through modifications to the inner sphere contribution $\left(R_{1}{ }^{\mathrm{IS}}\right)$ to the observed longitudinal relaxation rate constant; the outer sphere contributions are fixed by limitations of realistic contrast agent design. From Equation 1.19 it can be seen that a limited number of parameters are available to affect $R_{1}{ }^{\text {IS }}$. These are; the hydration number of the $\mathrm{Gd}^{3+}$ ion, the inner sphere water proton relaxation time constant $\left(T_{1 \mathrm{M}}\right)$ and the lifetime of an inner sphere coordinated water molecule, $\tau_{\mathrm{M}}$.

\subsubsection{Hydration state (q)}

All clinical $\mathrm{Gd}^{3+}$-based CAs have one metal bound water molecule and therefore are $\mathrm{q}=$ 1 agents. Opening up additional coordination sites to water by reducing the denticity of the ligand can lead to increases in relaxivity. However, this is often at the expense of increased agent toxicity, a result of decreased selectivity of the ligand for $\mathrm{Gd}^{3+}$ versus endogenous metals rather than directly related to stability. ${ }^{52}$ In addition to their decreased stability, agents with reduced ligand denticity are more prone to binding endogenous anions, such as phosphate, that displaces the inner sphere water leading to decreased relaxivities. ${ }^{53}$ Reports have recently been made of chelates in which $\mathrm{q}=2 .{ }^{54-55}$ However, it is unclear that these chelates would be sufficiently stable for human use. Therefore, the hydration state is essentially fixed at one. 


\subsubsection{Inner sphere water proton relaxation time constant $\left(T_{I M}\right)$}

To understand the factors that influence the inner sphere water proton relaxation time constant $\left(T_{1 \mathrm{M}}\right)$ further analysis of SBM theory is required. $T_{1 \mathrm{M}}$ has been found to be highly dependent on the longitudinal $\left(\tau_{\mathrm{c} 1}\right)$ and transverse $\left(\tau_{\mathrm{c} 2}\right)$ correlation time constants, and on $r_{\mathrm{Gd}-\mathrm{H}}{ }^{-6}$, the distance between the metal ion and the coordinated water proton (Equation 1.20). The latter is comparable for all clinical $q=1$ agents and has historically been considered to be fixed. All other components in Equation 1.20 are constants where; $\mu_{0}$ is the permeability of free space, $\hbar$ is the reduced Plancks constant equal to $h / 2 \pi, \gamma_{\mathrm{H}}$ and $\gamma_{\mathrm{S}}$ are the gyromagnetic ratios of a proton and electron respectively, $S$ is the electron spin quantum number, $\omega_{\mathrm{H}}$ and $\omega_{\mathrm{S}}$ are the proton and electronic Larmor frequencies respectively.

$$
\frac{1}{T_{1 M}}=R_{1 M}=\frac{2}{15}\left(\frac{\mu_{0}}{4 \pi}\right)^{2} \frac{h^{2} \gamma_{H}^{2} \gamma_{S}^{2}}{r_{G d H}^{6}} S(S+1)\left[\frac{3 \tau_{c 1}}{1+\omega_{H}^{2} \tau_{c 1}^{2}}+\frac{7 \tau_{c 2}}{1+\omega_{S}^{2} \tau_{c 2}^{2}}\right]
$$

$\tau_{\mathrm{c} 1}$ and $\tau_{\mathrm{c} 2}$ are themselves dependent on the rotational correlation time constant $\left(\tau_{\mathrm{R}}\right)$, the water residence life time $\left(\tau_{\mathrm{M}}\right)$ and the longitudinal and transverse electron relaxation time constants ( $\tau_{\mathrm{S} 1}$ and $\tau_{\mathrm{S} 2}$ respectively) as shown in Equation 1.21. Control of the later is less well understood and values for a given chelate have to be accepted.

$$
\frac{1}{\tau_{c i}}=\frac{1}{\tau_{R}}+\frac{1}{\tau_{M}}+\frac{1}{\tau_{S i}} \quad i=1,2
$$

Using these equations and fixing values for $\mathrm{c}, q, r_{\mathrm{Gd}-\mathrm{H}}$ and $\tau_{\mathrm{S}}$, the effect of $\tau_{\mathrm{R}}$ and $\tau_{\mathrm{M}}$ on relaxivity can be modeled. Such models show $\tau_{\mathrm{R}}$ and $\tau_{\mathrm{M}}$ values are key parameters for 
relaxivity enhancement. A relaxivity plot as a function of these two critical parameters at a fixed field strengths is often published. Figure 1.16 is typical of such plots and demonstrates the effect of $\tau_{\mathrm{R}}$ and $\tau_{\mathrm{M}}$ for a generic $\mathrm{Gd}^{3+}$ chelate at $0.47 \mathrm{~T}(20 \mathrm{MHz})$.

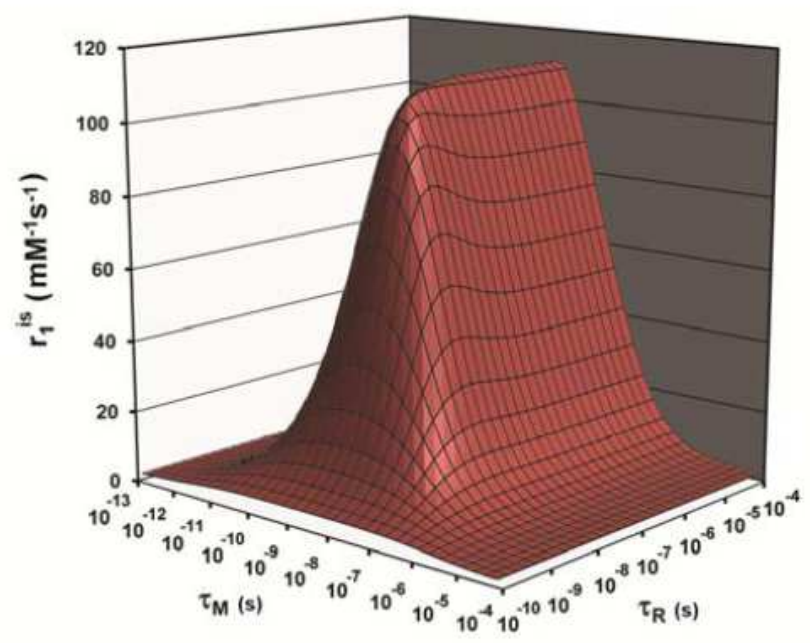

Figure 1.16: 3D simulation illustrating the effect of the rotational correlation time $\left(\tau_{R}\right)$ and the residence lifetime of the water molecule $\left(\tau_{\mathrm{M}}\right)$ coordinated to a metal ion for a generic $\mathrm{Gd}^{3+}$ chelate at $0.47 \mathrm{~T}(20 \mathrm{MHz})$.

\subsubsection{Rotational correlation time constant $\left(\tau_{R}\right)$}

Bulky agents restrict the ability of the molecule to tumble in space resulting in lengthened $\tau_{\mathrm{R}}$ values. This has a favorable outcome in terms of relaxivity gains but their increased size also restricts movement from the vasculature to the interstitial space. $\tau_{\mathrm{R}}$ is optimized when the rotational frequency is comparable to the proton larmor frequency at the imaging field strength. Most clinical imaging is performed at $1.41-3 \mathrm{~T}(60-128 \mathrm{MHz})$ therefore the GHz $\tau_{\mathrm{R}}$ values for clinically available $\mathrm{q}=1$ low molecular weight agents ${ }^{56}$ 
are too fast, requiring their rotation to be slowed to become more optimum, such as in the case of Ablavar (Figure 1.12).

Ablavar consists of a stable gadopentate core, with a phosphodiester linkage to a lipophilic albumin binding group. On binding (non-covalently) to human serum albumin (HSA) the effective molecular weight increases substantially and thus $\tau_{R}$ increases resulting in an increase in relaxivity. Several derivatives of this type of CA have been investigated including variants covalently bound to large macro and supramolecular carriers. $^{57}$

\subsubsection{Water residence life time ( $\left.\tau_{M}\right)$}

The time a water molecule spends associated with the $\mathrm{Gd}^{3+}$ ion is its water residence lifetime $\left(\tau_{\mathrm{M}}\right)$. The inverse of which describes the exchange kinetics of the inner sphere water molecules. If the water molecule is coordinated for too short a time before being exchanged with a bulk water molecule, and catalyze the PRE effect, then the water proton will not be fully relaxed and optimum relaxivity will take longer to reach. Conversely, if the water molecule remains associated fully relaxed for too long before being transferred to the bulk (as is the case for clinical $\mathrm{Gd}^{3+}$-based CA) then the efficiency of the relaxation is poor and relaxivity is not optimum. Investigations reveal that the optimum value of $\tau_{\mathrm{M}}$ for clinical agents is approximately $6 \mathrm{~ns}$ at the clinically relevant field strength of $1.41 \mathrm{~T}$ $(60 \mathrm{MHz})^{58}$ compared to about $20-40 \mathrm{~ns}$ at $0.47 \mathrm{~T}(20 \mathrm{MHz})$. 


\subsubsection{Electron relaxation time constants $\left(\tau_{S 1}\right.$ and $\left.\tau_{S 2}\right)$}

Although the effect of electron spin relaxation time constants, $\tau_{\mathrm{S} 1}$ and $\tau_{\mathrm{S} 2}$, on relaxivity is known ${ }^{59-60}$ and can be predicted by SBM theory, methods to accurately control their values are less well understood. According to experimental observations, increased rigidity and symmetry within a chelate, such as those based on the cyclic ligand DOTA, gives rise to better electron relaxation than with chelates based on the linear ligand DTPA, particularly for complexes that tumble slowly. ${ }^{22,61}$ This latter point makes them field dependent parameters as a result of the dependence of $\tau_{\mathrm{S} 1}$ and $\tau_{\mathrm{S} 2}$ on the zero-field splitting (ZFS) and is characterized by the correlation time $\left(\tau_{v}\right)$ expressed by the square of the trace of the ZFS tensor, $\Delta$, as shown in Equations 1.22 and 1.23 respectively. All other terms are constants as described previously.

$$
\begin{aligned}
& {\frac{1}{\tau_{S 1}}}^{Z F S}=\frac{12}{5} \Delta^{2} \tau_{v}\left[\frac{1}{1+\omega_{S}^{2} \tau_{v}^{2}}+\frac{4}{1+4 \omega_{S}^{2} \tau_{v}^{2}}\right] \\
& {\frac{1}{\tau_{S 2}}}^{Z F S}=\frac{12}{10} \Delta^{2} \tau_{v}\left[3+\frac{5}{1+\omega_{S}^{2} \tau_{v}^{2}}+\frac{2}{1+4 \omega_{S}^{2} \tau_{v}^{2}}\right]
\end{aligned}
$$

\subsection{Spin locking for recovering lost relaxivity}

In spite of the relatively sound understanding of all that has been discussed above, relaxivities of $\mathrm{Gd}^{3+}$-based CAs at clinical imaging fields are significantly lower than predicted by theory (Figure 1.15). Moreover, these values are much reduced from those obtainable at lower fields and continue to decrease as field strength increases. Therefore, 
a method to "recover" lost relaxivity from current clinically approved agents at high field strengths that retain SNR advantages could be adopted into clinical practice rapidly and would be of tremendous importance.

\subsubsection{Theory of spin locking}

The technique was initially investigated as an NMR technique in the 1950 's, ${ }^{62}$ but developed for imaging 30 years later $^{63}$ where it has been used extensively in nonenhancing tissue since. ${ }^{64-69}$ Spin locking refers to the application of a lower power, longer duration RF pulse $\left(B_{1}\right)$ in the $\mathrm{x}, \mathrm{y}$-plane phase shifted from a $90^{\circ} B_{1}$ excitation pulse such that it is parallel to the net magnetization. The purpose of which is to "lock" the magnetization into lower precessional frequencies around an "effective" field ( $\left.B_{\text {eff }}\right)$ that is reduced with respect to $B_{0}$ but maintains the high field advantages required for imaging. When considered in the rotating frame of reference, longitudinal relaxation of the magnetization against $\mathrm{B}_{\mathrm{eff}}$ exhibits time constants $\left(T_{1 \rho}\right)$ representative of those at the lower precessional frequencies induced by the spin locking pulse and this has implications for increasing CA detectability.

\subsubsection{Determination of rotating frame longitudinal relaxation time constants $\left(T_{1 \rho}\right)$}

$T_{1 \rho}$ values are determined using a spin lock pulse sequence (Figure 1.17). An initial $90^{\circ}$ $\mathrm{RF}$ excitation pulse along the $+\mathrm{x}$ axis $\left(B_{1(90) \mathrm{x}}\right)$ (1) converts the net magnetization into the $\mathrm{x}, \mathrm{y}$-plane (2). Directly after which, the $B_{1(90) \mathrm{x}}$ is turned off and a lower power, long duration $90^{\circ}$ spin lock RF pulse phase shifted by $90^{\circ}\left(B_{1(90) y}\right)$ is turned on for a period of 
time $(\tau)$ known as the locking time (TL) and generates an effective field ( $B_{\text {eff }}$ ). Throughout the duration of the spin lock pulse the net magnetization is locked in the $\mathrm{x}, \mathrm{y}-$ plane and undergoes longitudinal relaxation in the absence of transverse relaxation. When the spin locking pulse is turned off the signal from transverse magnetization in the $\mathrm{x}, \mathrm{y}-$ plane is detected (3). A relaxation delay time (RD) allows full relaxation of the magnetization back to equilibrium to occur prior to the next iteration of the pulse sequence (1) with a longer locking time $(\tau)$.

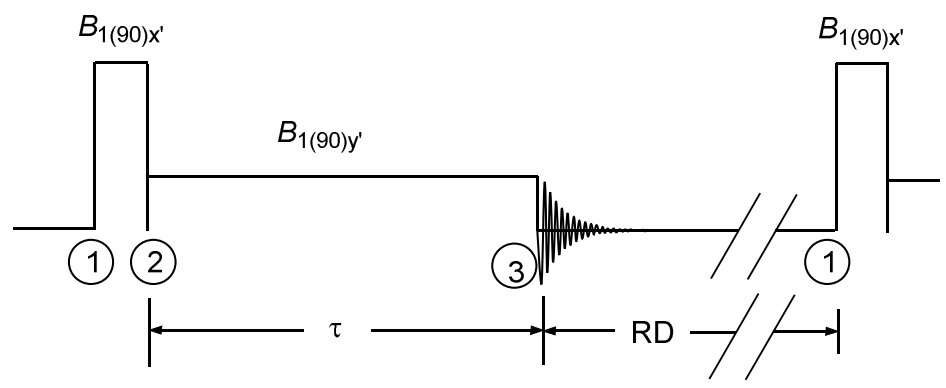

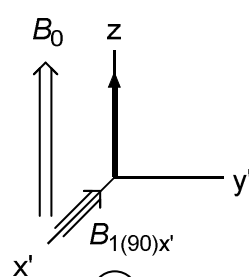

(1)

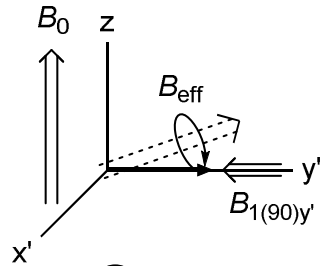

(2)

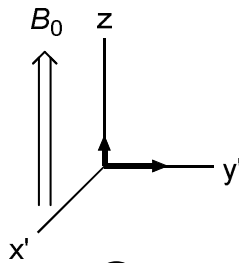

(3)

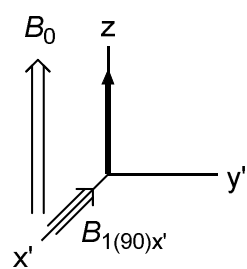

(1)

Figure 1.17: Spin lock pulse timing diagram (top), course and position of the net magnetization (bottom).

$T_{1 \rho}$, the longitudinal relaxation time constant in the rotating frame measured as the decay of transverse magnetization, is determined by plotting the signal intensity (S) of the peak maximum in the frequency domain at (3) (Figure 1.18, top) as a function of $\tau$, the spin locking time (Figure 1.18, bottom). As for $T_{2}, T_{1 \rho}$ is the time required for magnetization 
in the $x, y-p l a n e$ to decay to $37 \%$ of its original value (1) and is calculated by fitting the acquired data to Equation 1.24 that describes the decay of magnetization from the $\mathrm{x}, \mathrm{y}-$ plane after a $90^{\circ}$ phase shifted SL pulse and $e^{-\frac{\tau}{T_{1 \rho}}}$ is the exponential decay function.

$$
M_{x y}(\tau)=M_{x y}(0) e^{-\frac{\tau}{T_{1 \rho}}}
$$

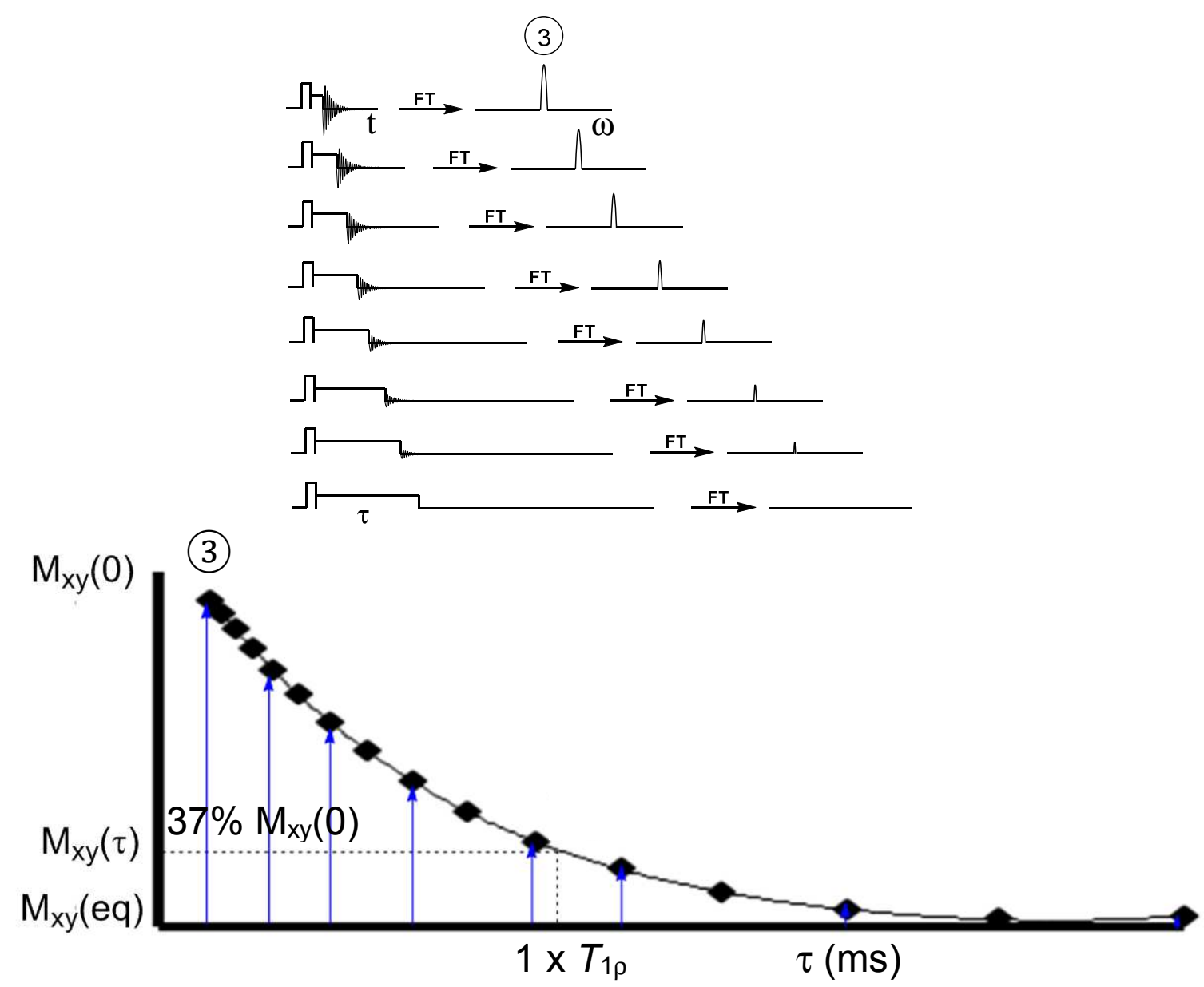

Figure 1.18: Summary of $T_{1 p}$ determination. Spin locking pulse sequence and generation of spectra (top) and the decay of magnetization ( $\left.M_{x y}\right)$ as a function of the variable spin lock duration

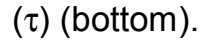




\subsection{Scope of this work: Determining the effect of a spin locking pulse on relaxivity}

The scope of this work is to determine the extent to which a spin locking pulse can "recover" lost relaxivity over a range of relatively high $B_{0}$ fields for a range of MRI contrast agents.

\subsubsection{Effect of pulse sequence parameters on $T_{1}$ and $T_{1} \rho$ time constants}

To compare relaxivities across several $B_{0}$ field strengths it is essential that relaxation time constants be obtained with parameters that offer the best possible fit of the data in order to generate reliable NMRD profiles. A number of pulse sequence parameters are available that potentially alter the reliability of a fit to observed relaxation data. Therefore, it was prudent to investigate the impact of deliberate variations to several pulse sequence parameters on the fitting of laboratory and rotating frames relaxation data and the subsequent time constants generated.

\subsubsection{Effect of spin lock pulse strength $\left(\gamma B_{1}\right)$ on longitudinal relaxation rate constants} $\left(R_{1 \rho}\right)$

Of the few reports relating to the rotating frame relaxation mechanism in the presence of paramagnetic ions, the work by Jordan and co-workers ${ }^{70}$ stands out. In it they describe the effect a hydrated paramagnetic ion would have on $R_{1 \rho}$ of bulk solvent nuclei. However, this paper did not investigate the effect of combining spin locking with paramagnetic $\mathrm{Gd}^{3+}$-based CAs. Unique to $\mathrm{Gd}^{3+}$ of the lanthanide metal ion series, $\mathrm{Gd}^{3+}$ 
does not induce a hyperfine shift $\left(\Delta \omega_{\mathrm{m}}^{2}\right)$. This invokes a condition identified by the authors that suggests there should be no significant change in $R_{1 \rho}$ as a function of the strength of the spin locking pulse $\left(\gamma B_{1}\right)$. A study to investigate this hypothesis is critical in order to generate accurate $r_{1 \rho}$ NMRD profiles for $\mathrm{Gd}^{3+}$-based CAs in which reliable comparison of data across several fields and instrument types can be made.

1.7.3. A comparison of laboratory $\left(r_{1}\right)$ and rotating $\left(r_{1} \rho\right)$ frame longitudinal relaxivities for $q=1$ clinical $G d^{3+}$-based CAs

The use of a low field spin locking pulse in the presence of a large $B_{0}$ field may allow for the generation of relaxivities in the rotating frame for current clinically available $\mathrm{Gd}^{3+}-$ based CAs higher than is presently obtainable in the laboratory frame. This has potential implications in any imaging applications that may require the administration of one of several $\mathrm{Gd}^{3+}$-based CAs clinically approved for MRI. Therefore a study to determine the extent of any increase in relaxivity by employing a spin locking pulse is critical to provide greater detail of the potential to improve agent detectability.

1.7.4. Effect of rotational correlation time $\left(\tau_{R}\right)$ on rotating frame longitudinal relaxivity $\left(r_{1 \rho}\right)$

It is also possible from the theory derived by Jordan et al. to hypothesize that $r_{1} \rho$ should be more sensitive to the rotational correlation time $\left(\tau_{R}\right)$ than $r_{1}$. This is due to the introduction of the laboratory frame transverse relaxation rate constant $\left(R_{2 \mathrm{M}}\right)$ into the 
description of $r_{1 \rho}$ and its dominance over the laboratory frame longitudinal relaxation rate constant $\left(R_{1 \mathrm{M}}\right)$. Nanoassembled capsules (NACs) prepared by Annah Farashishiko in our group that contain a $\mathrm{q}=0$ chelate $\left(\mathrm{GdDOTP}^{5-}\right)$ bound to polymer and encapsulated in $\mathrm{SiO}_{2}$ are a useful model for studying the effect slowly tumbling agents with long $\tau_{\mathrm{R}}$ values have on rotating frame relaxivities. A study to investigate the effect of spin locking on the rotating frame relaxivities of NACs was performed and results compared to the laboratory frame relaxivities over a range of $B_{0}$ fields.

\subsubsection{Effect of hydration number (q) on rotating frame longitudinal relaxivity $\left(r_{1 \rho}\right)$}

It is well established that the laboratory frame longitudinal relaxivity $\left(r_{1}\right)$ of $\mathrm{Gd}^{3+}$-based CAs scales with hydration number $(q)$ at the expense of the thermodynamic stability of the chelate. On opening up an extra coordination site to water, stability reduces by up to as much as three orders of magnitude limiting the choice of agents for practical in vivo use to those with a hydration number of just one. ${ }^{71}$ However, for ex vivo and post mortem investigations stability of the CA is not a concern. Applications such as these were just the reason we needed, other than for fundamental purposes, to study the effect hydration number has on the rotating frame longitudinal relaxivity $\left(r_{1 \rho}\right)$. 


\section{CHAPTER 2. EFFECT OF PULSE SEQUENCE PARAMETERS ON LONGITUDINAL ( $\left.T_{1}\right)$ AND ROTATING ( $\left.T_{1 \rho}\right)$ FRAME TIME CONSTANTS}

\subsection{Establishing pertinent pulse sequence parameters with potential to effect reliability of fit of relaxation data}

To compare relaxivities across several $B_{0}$ field strengths it is essential that relaxation time constants be obtained with parameters that offer the best possible fit of the data in order to generate reliable NMRD profiles. A number of pulse sequence parameters are available that potentially alter the reliability of a fit to observed relaxation data. Herein, we investigate the effect deliberate variations to pertinent pulse sequence parameters have on the fitting of observed time curve data and the $T_{1}$ and $T_{1 \rho}$ values generated. For this experiment, a PBS solution of Dotarem with a $\mathrm{Gd}^{3+}$ concentration of $25 \mathrm{mM}$ was used and single point $(\mathrm{n}=1)$ measurements taken. For each parameter and temperature investigated, the observed variance (standard deviation) in the time constants generated over the range of deliberate variations were compared to the natural variance (standard deviation) for a sample prepared in the same manner. The natural standard deviation was determined from replicate analysis $(n=20)$ on the same sample using a fixed set of parameter values. We limited the investigation to our $60 \mathrm{MHz}(1.41 \mathrm{~T})$ fixed low field relaxometer to reflect current clinical imaging field strengths. Measurements were made at historically and physiologically relevant temperatures, 25 and $37{ }^{\circ} \mathrm{C}$ respectively. Time constants generated from time curves at 25 and $37^{\circ} \mathrm{C}$ are presented and discussed below. For clarity, a discussion of time curve fitting was restrained to time curves 
generated at $25^{\circ} \mathrm{C}$, however, comparable fitting and trends were observed for time curves generated at $37^{\circ} \mathrm{C}$ and can be found in appendix 1 .

The pulse sequence parameters investigated were:

\subsubsection{Relaxation delay $(R D)$ time}

The relaxation delay (RD) time defines the time between the detection of a signal and the subsequent RF pulse of the next scan or iteration of the pulse sequence and should allow sufficient time for full relaxation of the magnetization back to equilibrium to occur.

\subsubsection{Pulse delay ( $\tau)$ times}

The pulse delay time defines the time magnetization is allowed to recover back to thermal equilibrium against $B_{0}$ and $B_{\text {eff }}$ for $T_{1}$ and $T_{1 \rho}$ measurements respectively prior to signal being detected. For $T_{1 \rho}$ measurements, this occurs in the presence of a spin lock pulse and is called the spin lock time (TL). The pulse delays should allow for a full range of signal intensities to be detected.

\subsubsection{Number of data points}

The number of data points (or pulse delays) dispersed across the range of pulse delays sampled determines how closely spaced in time the pulse delays are. The number should be appropriate for the type of exponential behavior expected and reduce instrument and operator time. 


\subsection{Establishment of ideal pulse sequence parameter values}

The following table identifies the suggested "ideal" values for the pulse sequence parameters to be investigated obtained from either the user manual ${ }^{72}$ or reported in literature. $^{73}$

Table 2.1: Suggested "ideal" pulse sequence parameter values for determinations of $T_{1}$ and $T_{1 p}$.

\begin{tabular}{c|c:c}
$\begin{array}{l}\text { Pulse sequence } \\
\text { parameter }\end{array}$ & $\begin{array}{l}\text { Inversion } \\
\text { recovery }\end{array}$ & Spin locking \\
\hline Relaxation delay time (s) & $=10 \times T_{1}$ & $\begin{array}{c}=10 \times T_{1} \\
\text { or minimum allowable } \geq 10 \times T_{1}\end{array}$ \\
\hline Pulse delays times (ms) & & $<1 / 10$ th $\times T_{1}$ \\
\hline First pulse delay & $<1 / 10$ th $\times T_{1}$ & $>5 \times T_{1}$ \\
\hline Last pulse delay & $>5 \times T_{1}$ & or maximum allowed (40 ms) \\
\hline $\begin{array}{l}\text { Number of pulse delays for } \\
\text { mono-exponential behavior }\end{array}$ & 16 & 16
\end{tabular}

\subsection{Other parameters and general considerations}

Several other parameters that a user would not typically alter but do affect the reliability of fit of relaxation data if not controlled are discussed below for completeness. These parameters were held constant for all experiments herein with the values stated where applicable. 


\subsubsection{Sample temperature, volume and position}

Relaxation time constants are temperature dependent, therefore, the sample was inserted into the coil of the probe head and equilibrated to the desired temperature for at least five minutes prior to any measurements being made. To maximize the signal-to-noise a sample volume sufficient to fill the RF receiver coil is recommended by the instrument manufacturer. A volume of $0.5 \mathrm{~mL}$ was employed. The sample was positioned safely within the built-in sample holder and the minispec operated on a benchtop that minimizes vibrations and reduces pulse angle errors.

\subsubsection{Pulse angles}

The angle by which the net magnetization is flipped is determined by the power and duration of the pulse. Pulse angles can be calibrated to ensure accurate flip angles are achieved. For these experiments, $90^{\circ}$ and $180^{\circ}$ pulse angles were achieved with pulse durations of 2.54 and $4.78 \mu$ s for 0 decibels attenuation. These values are provided by the minispec software. however, at the time of writing are thought to be wrong.

\subsubsection{Offset frequency}

The offset resonance frequency should be equal to the resonance (Larmor) frequency of the water protons determined by the spectrometer, i.e. on-resonance. 


\subsubsection{Number of scans}

The number of scans for all experiments was 4,1 scan per phase, the minimum required when the phase cycling option is selected.

\subsubsection{Receiver gain, detection mode and fitting equations}

Receiver gain was set to the factory installed setting (-66 dB). For $T_{1}$ determinations the "Real" detection mode was used. For $T_{1 \rho}$ determination the "Magnitude" detection mode was used to allow the full range of signal to be observed. A mono-exponential behavior was expected, therefore, fitting to the relevant exponential equation (Equation 1.12 and 1.24) was satisfactory.

\subsection{Determination of natural variance (standard deviation) from replicate analysis}

Using the suggested "ideal" pulse sequence parameter values in Table 2.1 replicate $(\mathrm{n}=$ 20) determinations of $T_{1}$ and $T_{1 \rho}$ were made on a single sample preparation of Dotarem in PBS pH 7.4 at a $\mathrm{Gd}^{3+}$ concentration of $25 \mathrm{mM}$. The time constants and the natural variance in the data (measured as standard deviation) for each time constant at both 25 and $37{ }^{\circ} \mathrm{C}$ are presented (Table 2.2). Figure 2.1 shows more clearly the measurement repeatability of the data. The difference due to temperature is a consequence of Dotarem, a small molecule, being in the fast exchange regime and having a short correlation time $\left(\tau_{c}\right)$. In this case, a decrease in temperature results in a decrease in the time constant due to a lengthening of the correlation time and more efficient dipole-dipole interactions. 


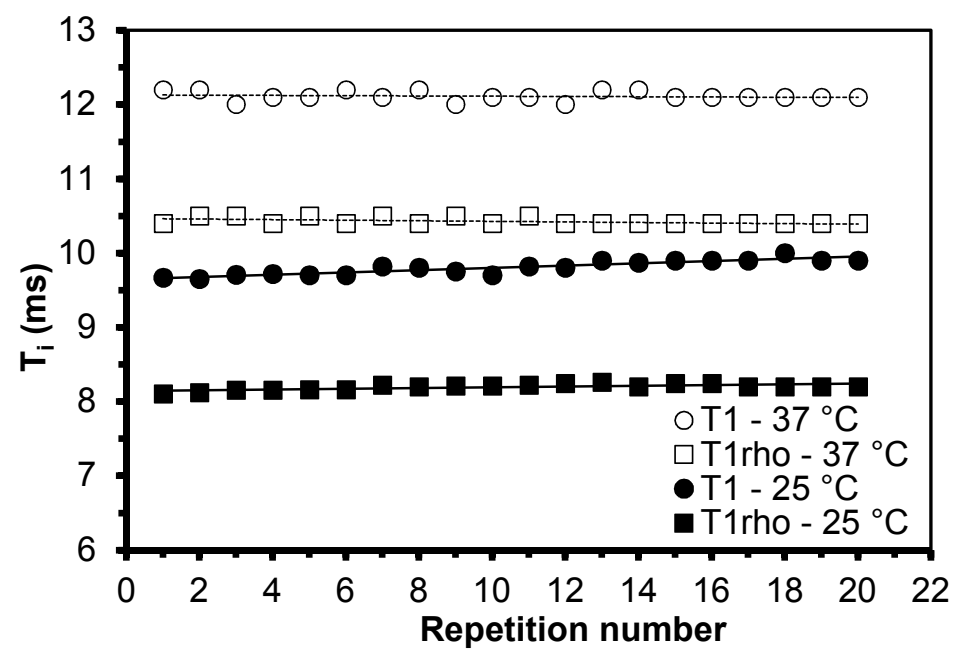

Figure 2.1: Determination of natural variance in $T_{1}$ and $T_{1 \rho}$ at $1.41 \mathrm{~T}(60 \mathrm{MHz})$ for a single sample preparation of Dotarem in PBS pH 7.4 with a $\mathrm{Gd}^{3+}$ concentration of $25 \mathrm{mM}$. The "ideal" pulse sequence parameters identified in Table 2.1 were used.

It can be seen from the data presented in Figure 2.1 and Table 2.2 that the natural variance in the repeatability data of $T_{1}$ and $T_{1 \rho}$ at both 25 and $37{ }^{\circ} \mathrm{C}$ is very low with standard deviations of 0.10 and $0.067 \mathrm{~ms}$ for $T_{1}$ measurements at 25 and $37{ }^{\circ} \mathrm{C}$ respectively and 0.042 and $0.047 \mathrm{~ms}$ for $T_{1 \rho}$ measurements at 25 and $37^{\circ} \mathrm{C}$ respectively.

The standard deviations for time constants obtained by changing pulse sequence parameter values shall be determined and compared to the standard deviations reported above, where the above represents the natural variance from replicate analysis. If the standard deviation for a series of time constants is greater than the standard deviation due to natural variation in the data then the variation in the time constant values will be considered significant and a result of the change to the parameter value. From this it will be possible to make recommendations to ensure reliable time constants. 
Table 2.2: Determination of natural variance in $T_{1}$ and $T_{1 \rho}$ at $1.41 \mathrm{~T}(60 \mathrm{MHz})$ for a single sample preparation of Dotarem in PBS pH 7.4 with a $\mathrm{Gd}^{3+}$ concentration of $25 \mathrm{mM}$. The "ideal" pulse sequence parameters identified in Table 2.1 were used. SD is the standard deviation.

\begin{tabular}{|c|c|c|c|c|}
\hline \multirow[b]{2}{*}{ Repetition \# } & \multicolumn{2}{|c|}{$T_{1}(\mathrm{~ms})$} & \multicolumn{2}{|c|}{$T_{1_{p}}(\mathrm{~ms})$} \\
\hline & $25^{\circ} \mathrm{C}$ & $37^{\circ} \mathrm{C}$ & $25^{\circ} \mathrm{C}$ & $37^{\circ} \mathrm{C}$ \\
\hline 1 & 9.67 & 12.2 & 8.10 & 10.4 \\
\hline 2 & 9.65 & 12.2 & 8.12 & 10.5 \\
\hline 3 & 9.71 & 12.0 & 8.15 & 10.5 \\
\hline 4 & 9.72 & 12.1 & 8.15 & 10.4 \\
\hline 5 & 9.7 & 12.1 & 8.16 & 10.5 \\
\hline 6 & 9.7 & 12.2 & 8.16 & 10.4 \\
\hline 7 & 9.82 & 12.1 & 8.2 & 10.5 \\
\hline 8 & 9.8 & 12.2 & 8.2 & 10.4 \\
\hline 9 & 9.75 & 12.0 & 8.2 & 10.5 \\
\hline 10 & 9.7 & 12.1 & 8.2 & 10.4 \\
\hline 11 & 9.82 & 12.1 & 8.2 & 10.5 \\
\hline 12 & 9.8 & 12.0 & 8.2 & 10.4 \\
\hline 13 & 9.9 & 12.2 & 8.3 & 10.4 \\
\hline 14 & 9.87 & 12.2 & 8.20 & 10.4 \\
\hline 15 & 9.9 & 12.1 & 8.24 & 10.4 \\
\hline 16 & 9.9 & 12.1 & 8.24 & 10.4 \\
\hline 17 & 9.9 & 12.1 & 8.20 & 10.4 \\
\hline 18 & 10.0 & 12.1 & 8.20 & 10.4 \\
\hline 19 & 9.9 & 12.1 & 8.20 & 10.4 \\
\hline 20 & 9.90 & 12.1 & 8.20 & 10.4 \\
\hline Natural variance (SD) & 0.10 & 0.067 & 0.042 & 0.047 \\
\hline
\end{tabular}




\subsection{Effect of pulse sequence parameters on the reliability of fit of relaxation data}

\subsubsection{Relaxation delay (RD) time}

With the inversion recovery pulse sequence it was noticed that with a relaxation delay time $<10 \times T_{1}$ the measured signal for short pulse delay times $(\tau)$ are offset by $\sim 20 \%$ (Figure 2.2, squares) compared to when the relaxation delay is $=10 \times T_{1}$ (diamonds), $>$ $10 \times T_{1}$ (triangles) or $>>10 \times T_{1}$ (crosses). This demonstrates that a relaxation delay time that is too short does not allow full relaxation back to equilibrium thus reducing the signal of the subsequent scan.

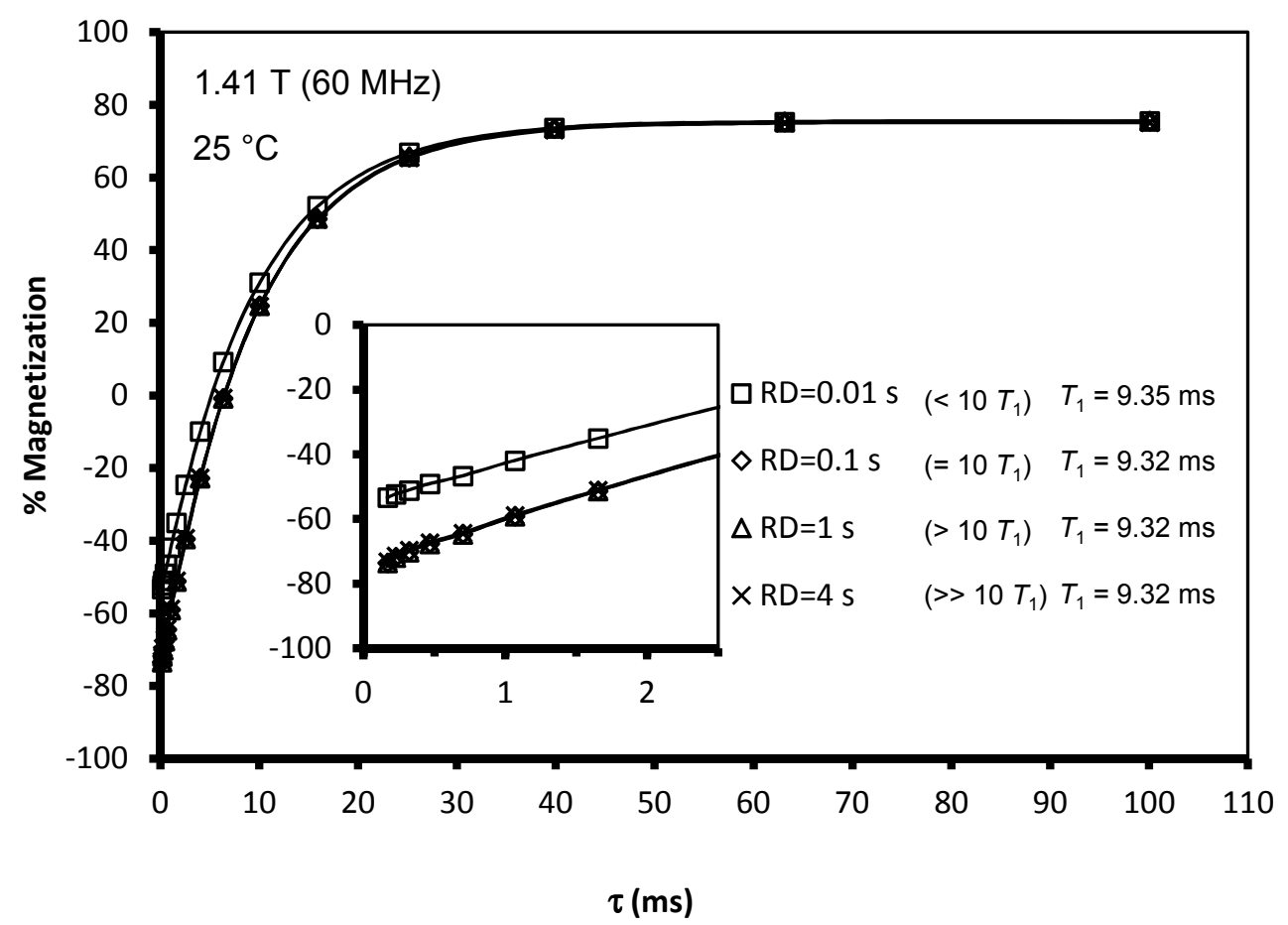

Figure 2.2: Effect of delibarate variations to the relaxation delay (RD) time on $T_{1}$ time curves (inset shows deviation (squares) when relaxation delay is $<10 \times \mathrm{T}_{1}$ ). Sample: PBS pH 7.4 solution of Dotarem, $\left[\mathrm{Gd}^{3+}\right]=25 \mathrm{mM} . \mathrm{n}=1$. 
With the spin locking pulse sequence almost identical time curves were generated (Figure 2.3) for all four RD times studied. This is due to the $\mathrm{RD}$ times employed all being $>>10$ $\times T_{1}$ due to a $4 \mathrm{~s}$ minimum imposed by the instrument to preserve hardware during the use of long pulse delays required to observe full signal decay.
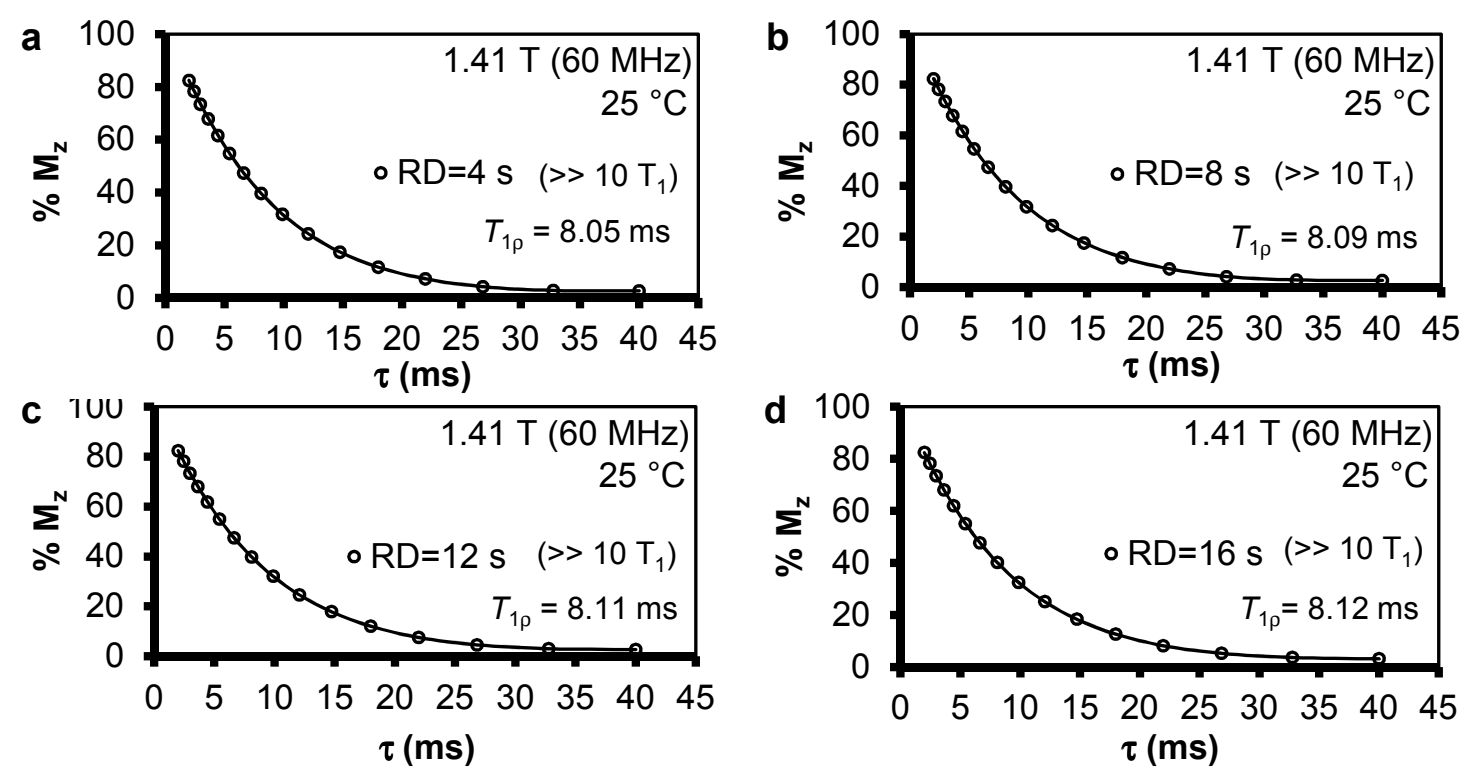

Figure 2.3: Effect of delibarate variations to the relaxation delay (RD) time on $T_{1 \rho}$ time curves. Sample: PBS pH 7.4 solution of Dotarem, $\left[\mathrm{Gd}^{3+}\right]=25 \mathrm{mM} . \mathrm{n}=1$.

The measured standard deviation for time constants generated here $(\mathrm{n}=1)$ from monoexponential fitting of the time curves are 0.015 and $0.00 \mathrm{~ms}$ for $T_{1}$ measurements at 25 and $37{ }^{\circ} \mathrm{C}$ respectively and 0.031 and $0.050 \mathrm{~ms}$ for $T_{1 \rho}$ measurements at 25 and $37{ }^{\circ} \mathrm{C}$ respectively (Table 2.3). All standard deviations are approximately equal or less than the natural variance (standard deviation) of the data. It can therefore be said that there was no significant effect on time constants by varying the relaxation delay time. 
Table 2.3: Effect of deliberate variations to the relaxation delay (RD) time on $T_{1}$ and $T_{1 \rho}$ values at $1.41 \mathrm{~T}(60 \mathrm{MHz})$. Sample: PBS pH 7.4 solution of Dotarem, $\left[\mathrm{Gd}^{3+}\right]=25 \mathrm{mM} .(\mathrm{n}=1)$.

\begin{tabular}{|c|c|c|c|c|c|}
\hline \multirow[b]{2}{*}{ RD time (s) } & \multicolumn{2}{|c|}{$T_{1}(\mathrm{~ms})$} & \multirow[b]{2}{*}{ RD time (s) } & \multicolumn{2}{|c|}{$T_{1 p}(\mathrm{~ms})$} \\
\hline & $25^{\circ} \mathrm{C}$ & $37^{\circ} \mathrm{C}$ & & $25^{\circ} \mathrm{C}$ & $37^{\circ} \mathrm{C}$ \\
\hline $0.01\left(<10 \times T_{1}\right)$ & 9.35 & 11.4 & $4\left(\gg 10 \times T_{1}\right)$ & 8.05 & 10.4 \\
\hline $0.1\left(=10 \times T_{1}\right)$ & 9.32 & 11.4 & $8\left(\gg 10 \times T_{1}\right)$ & 8.09 & 10.3 \\
\hline $1\left(>10 \times T_{1}\right)$ & 9.32 & 11.4 & $12\left(\gg>10 \times T_{1}\right)$ & 8.11 & 10.3 \\
\hline $4\left(\gg>10 \times T_{1}\right)$ & 9.32 & 11.4 & $16\left(\gg>10 \times T_{1}\right)$ & 8.12 & 10.3 \\
\hline $\begin{array}{l}R D \text { variance } \\
\text { (SD) }\end{array}$ & 0.015 & 0.00 & & 0.031 & 0.050 \\
\hline $\begin{array}{l}\text { Natural variance } \\
\text { (SD) }\end{array}$ & 0.10 & 0.067 & & 0.042 & 0.047 \\
\hline
\end{tabular}

\subsubsection{Pulse delay $(\tau)$ times}

By increasing the duration of the first pulse delay time (Figure 2.4) in the inversion recovery and spin lock pulse sequences a smaller proportion of the relaxation time curve is observed. This is due to relaxation occurring at a rate relatively fast compared to the timing of the first pulse delay time. With a first pulse delay time of $0.01 \mathrm{~ms}\left(<<1 / 10^{\text {th }} \times\right.$ $\left.T_{1}\right)$ and $0.2 \mathrm{~ms}\left(<1 / 10^{\text {th }} \times T_{1}\right)$ for the determination of $T_{1 \rho}$ the measured signal for short pulse delay times $(\tau)$ are scattered (Figure $2.4 \mathrm{~d}$ and $2.4 \mathrm{e}$ ). This is the result of small coherences that remain in the transverse plane that are unable to relax during very short delay times resulting in errors of the time constant estimation. On increasing the duration of the first pulse delay time to $1 \mathrm{~ms}\left(=1 / 10^{\text {th }} \times T_{1}\right)$ and $2 \mathrm{~ms}\left(>1 / 10^{\text {th }} \times T_{1}\right)$ this scatter is avoided at the cost of at most $\sim 10 \%$ of the time curve (Figure $2.4 \mathrm{f}$ and $2.4 \mathrm{~g}$ ). 

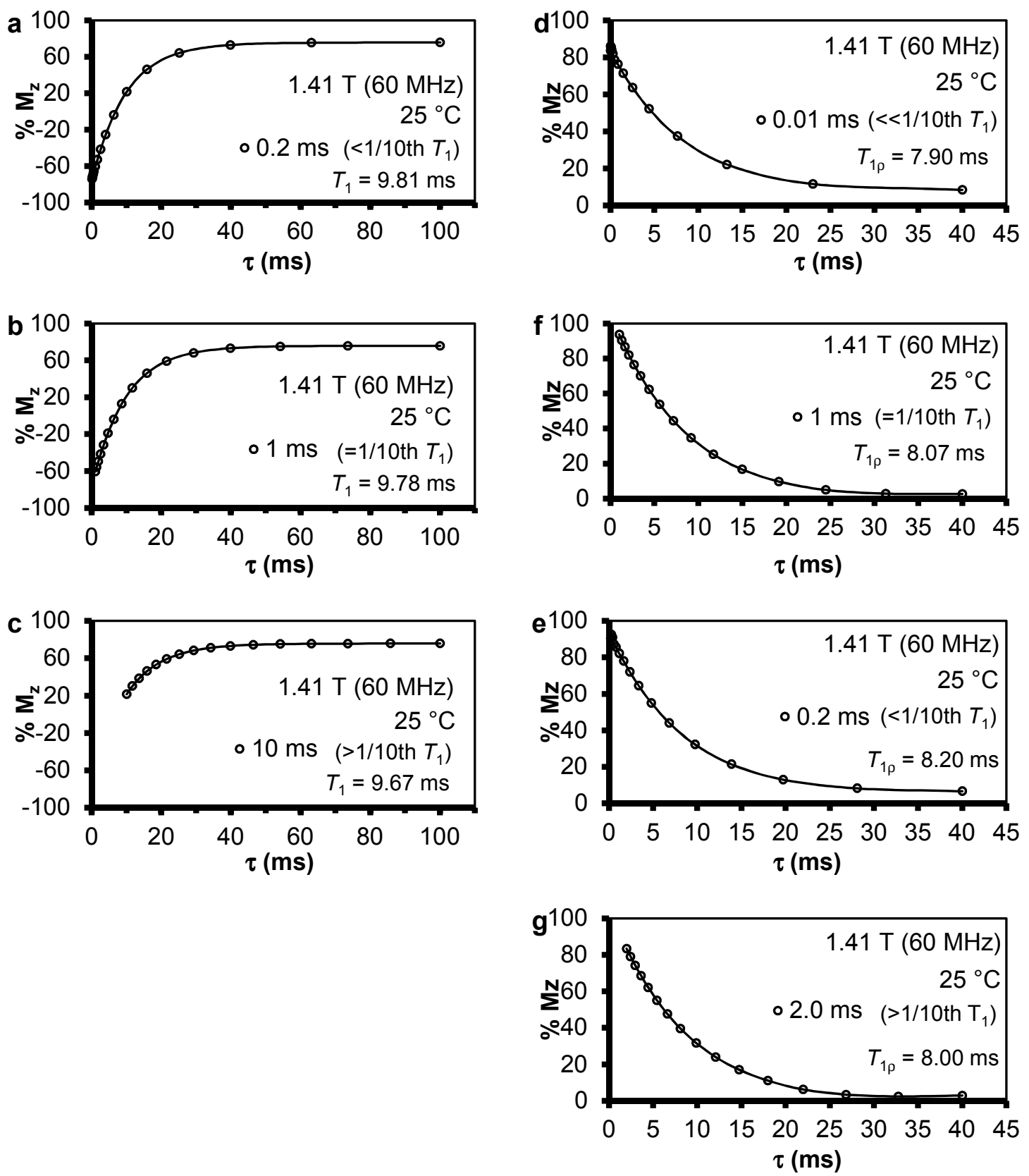

Figure 2.4: Effect of deliberate variations to the first pulse delay time $(\tau)$ on $T_{1}(\mathrm{a}-\mathrm{c})$ and $T_{1 \mathrm{p}}(\mathrm{d}-$ g) time curves. Sample: PBS pH 7.4 solution of Dotarem, $\left[\mathrm{Gd}^{3+}\right]=25 \mathrm{mM} . \mathrm{n}=1$.

Similarly, by decreasing the duration of the last pulse delay time (Figure 2.5) in the inversion recovery and spin lock pulse sequences a smaller proportion of the relaxation 
time curve is observed. This is due to not employing sufficiently long pulse delay times to observe the full return of signal to equilibrium.
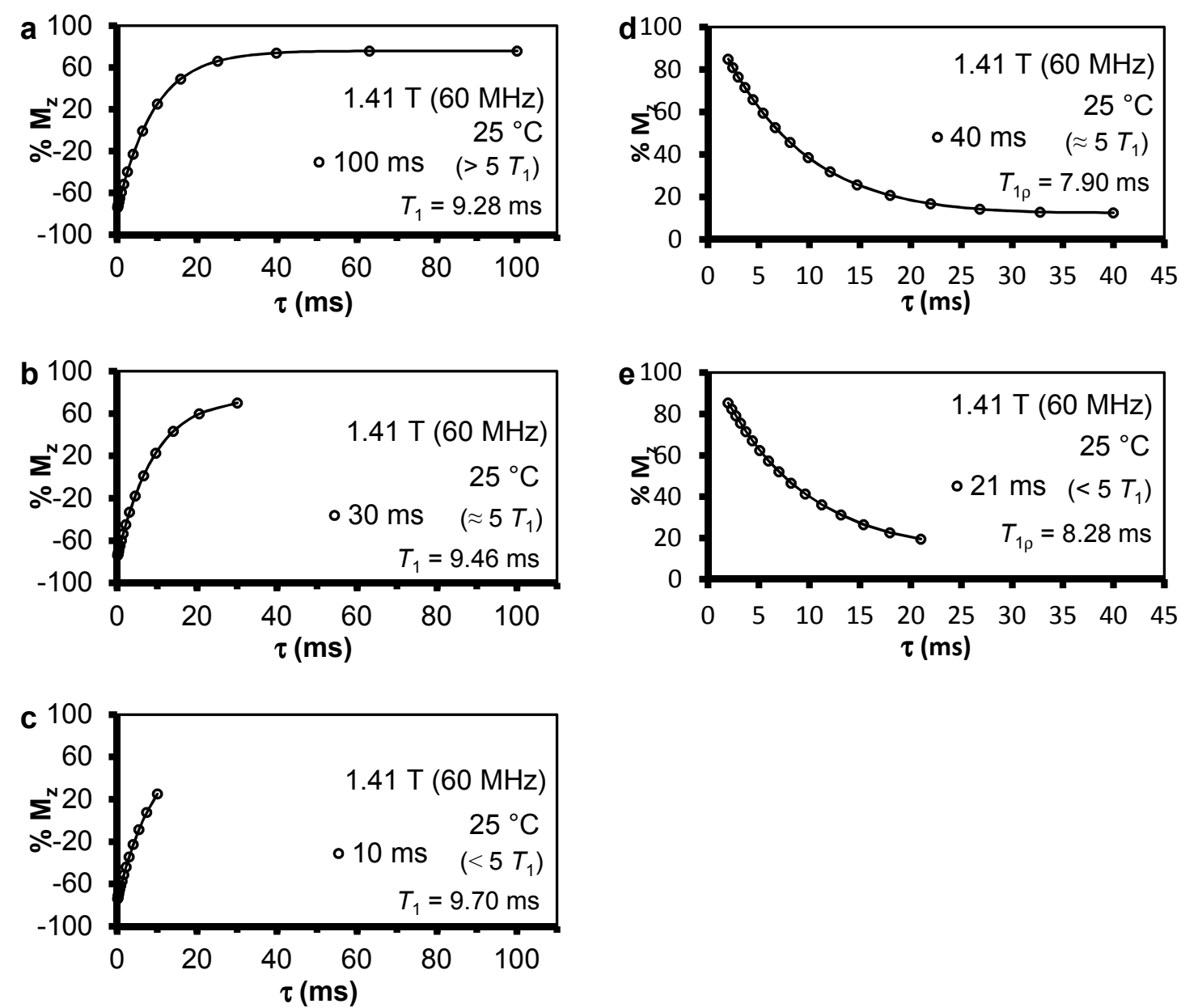

Figure 2.5: Effect of deliberate variations to the last pulse delay time $(\tau)$ on $T_{1}(\mathrm{a}-\mathrm{c})$ and $T_{1 \mathrm{p}}(\mathrm{d}-$ e) time curves. Sample: PBS pH 7.4 solution of Dotarem, $\left[\mathrm{Gd}^{3+}\right]=25 \mathrm{mM} . \mathrm{n}=1$.

The measured standard deviation for time constants generated here $(\mathrm{n}=1)$ from monoexponential fitting of the time curves for first pulse delay times are 0.082 and $0.16 \mathrm{~ms}$ for $T_{1}$ measurements at 25 and $37{ }^{\circ} \mathrm{C}$ respectively and 0.13 and $0.32 \mathrm{~ms}$ for $T_{1 \rho}$ measurements at 25 and $37{ }^{\circ} \mathrm{C}$ respectively. For last pulse delay times the range 
differences are 0.21 and $0.53 \mathrm{~ms}$ for $T_{1}$ measurements at 25 and $37{ }^{\circ} \mathrm{C}$ respectively and 0.27 and $0.16 \mathrm{~ms}$ for $T_{1 \rho}$ measurements at 25 and $37^{\circ} \mathrm{C}$, respectively (Table 2.4).

All standard deviations are larger than the natural variance (standard deviation) in the data with the exception of $T_{1}$ values at $25{ }^{\circ} \mathrm{C}$ when varying the duration of the first pulse delay time. It can therefore be said that, generally, there was a significant effect on time constants by varying the relaxation delay time.

\subsubsection{Number of data points}

An increased number of data points decreases the time between sampling of the magnetization and allows for more data points around the inflection of the time curve (Figure 2.6).

The measured standard deviation for time constants generated here $(\mathrm{n}=1)$ from monoexponential fitting of the time curves are 0.047 and $0.00 \mathrm{~ms}$ for $T_{1}$ measurements at 25 and $37{ }^{\circ} \mathrm{C}$ respectively and 0.026 and $0.024 \mathrm{~ms}$ for $T_{1 \rho}$ measurements at 25 and $37{ }^{\circ} \mathrm{C}$ respectively (Table 2.5). All standard deviations are less than the natural variance (standard deviation) in the data. It can therefore be said that there was no significant effect on time constants by varying the number of data points.

\subsection{Recommendations}

Often the expected $T_{1}$ or $T_{1 \rho}$ is unknown and pulse sequence parameters have to be chosen based on a best estimate of the time constants and subsequently refined. The 
following recommendations provide a starting point for initial experiments and should allow for reliable time constants to be generated. These are summarized in Table 2.6.

Table 2.4: Effect of delibarate variations to the first and last pulse delay $(\tau)$ times on $T_{1}$ and $T_{1}$ values at $1.41 \mathrm{~T}(60 \mathrm{MHz})$. Sample: PBS pH 7.4 solution of Dotarem, $\left[\mathrm{Gd}^{3+}\right]=25 \mathrm{mM} .(\mathrm{n}=1)$.

\begin{tabular}{|c|c|c|c|c|c|}
\hline \multirow[b]{2}{*}{$\begin{array}{l}\text { First delay time }(\tau) \\
\qquad(\mathrm{ms})\end{array}$} & \multicolumn{2}{|c|}{$T_{1}(\mathrm{~ms})$} & \multirow[b]{2}{*}{$\begin{array}{l}\text { First delay time }(\tau) \\
\qquad(\mathrm{ms})\end{array}$} & \multicolumn{2}{|c|}{$T_{1 \rho}(\mathrm{ms})$} \\
\hline & $25^{\circ} \mathrm{C}$ & $37^{\circ} \mathrm{C}$ & & $25^{\circ} \mathrm{C}$ & $37^{\circ} \mathrm{C}$ \\
\hline $0.2\left(<1 / 10^{\text {th }} \times T_{1}\right)$ & 9.81 & 11.1 & $0.01\left(<<1 / 10^{\text {th }} \times T_{1}\right)$ & 7.90 & 9.20 \\
\hline $1\left(=1 / 10^{\text {th }} \times T_{1}\right)$ & 9.78 & 11.2 & $0.2\left(<1 / 10^{\text {th }} \times T_{1}\right)$ & 8.20 & 9.70 \\
\hline $10\left(>1 / 10^{\text {th }} \times T_{1}\right)$ & 9.67 & 10.9 & $1\left(=1 / 10^{\text {th }} \times T_{1}\right)$ & 8.07 & 9.83 \\
\hline- & - & - & $2\left(>1 / 10^{\text {th }} \times T_{1}\right)$ & 8.00 & 9.91 \\
\hline $\begin{array}{l}\text { First delay variance } \\
\text { (SD) }\end{array}$ & 0.082 & 0.16 & & 0.13 & 0.32 \\
\hline $\begin{array}{l}\text { Natural variance } \\
\text { (SD) }\end{array}$ & 0.10 & 0.067 & & 0.042 & 0.047 \\
\hline
\end{tabular}

\begin{tabular}{|c|c|c|c|c|c|}
\hline \multirow[b]{2}{*}{$\begin{array}{l}\text { Last delay time }(\tau) \\
\quad(\mathbf{m s})\end{array}$} & \multicolumn{2}{|c|}{$T_{1}(\mathrm{~ms})$} & \multirow[b]{2}{*}{$\begin{array}{l}\text { Last delay time }(\tau) \\
\qquad(\mathrm{ms})\end{array}$} & \multicolumn{2}{|c|}{$T_{1 \rho}(\mathrm{ms})$} \\
\hline & $25^{\circ} \mathrm{C}$ & $37^{\circ} \mathrm{C}$ & & $25^{\circ} \mathrm{C}$ & $37^{\circ} \mathrm{C}$ \\
\hline $10\left(<5 \times T_{1}\right)$ & 9.70 & 10.0 & $21\left(<5 \times T_{1}\right)$ & 8.28 & 10.3 \\
\hline $30\left(\cong 5 \times T_{1}\right)$ & 9.46 & 10.8 & $40\left(\cong 5 \times T_{1}\right)$ & 7.90 & 10.1 \\
\hline $100\left(>5 \times T_{1}\right)$ & 9.28 & 11.0 & - & - & - \\
\hline $\begin{array}{l}\text { Last delay variance } \\
\text { (SD) }\end{array}$ & 0.21 & 0.53 & & 0.27 & 0.16 \\
\hline $\begin{array}{l}\text { Natural variance } \\
\text { (SD) }\end{array}$ & 0.10 & 0.067 & & 0.042 & 0.047 \\
\hline
\end{tabular}


Table 2.5: Effect of delibarate variations to the number of data points on $T_{1}$ and $T_{1 p}$ values at $1.41 \mathrm{~T}(60 \mathrm{MHz})$. Sample: PBS pH 7.4 solution of Dotarem, $\left[\mathrm{Gd}^{3+}\right]=25 \mathrm{mM} .(\mathrm{n}=1)$.

\begin{tabular}{c|c:c|c|c} 
& \multicolumn{2}{|c|}{$\boldsymbol{T}_{\mathbf{1}} \mathbf{( \mathbf { m s } )}$} & \multicolumn{2}{c}{$\boldsymbol{T}_{\mathbf{1}}$ ( $\left.\mathbf{m s}\right)$} \\
\hline \# data points & $\mathbf{2 5}{ }^{\circ} \mathbf{C}$ & $\mathbf{3 7}{ }^{\circ} \mathbf{C}$ & $\mathbf{2 5}{ }^{\circ} \mathbf{C}$ & $\mathbf{3 7}{ }^{\circ} \mathbf{C}$ \\
\hline $\mathbf{8}$ & 9.80 & 11.4 & 8.10 & 9.80 \\
$\mathbf{1 6}$ & 9.75 & 11.4 & 8.10 & 9.85 \\
$\mathbf{3 2}$ & 9.69 & 11.4 & 8.15 & 9.80 \\
$\mathbf{6 4}$ & 9.77 & 11.4 & 8.14 & 9.81 \\
\hline \# data point (SD) & 0.047 & 0.00 & 0.026 & 0.024 \\
\hline Natural variance (SD) & 0.10 & 0.067 & 0.042 & 0.047
\end{tabular}

\subsubsection{Relaxation delay (RD) times}

It has been demonstrated that time constants were not affected significantly (standard deviation was less than the standard deviation due to natural variation in the data) by the relaxation delay times examined including those less than the suggested ideal value of 10 $\times T_{1}$ where the magnetization range covered becomes narrower. However, a relaxation delay time of $10 \times T_{1}$ is more conventional to ensure full relaxation before subsequent scans and is therefore recommended. It should be noted that when using the spin locking pulse sequence long pulse delay times are sometimes necessary to observe the full relaxation time curve. This necessitates long relaxation delay times which are automatically imposed by the instrument and are typically much greater than $10 \times T_{1}$. In these cases, the minimum allowable relaxation delay time should be used to avoid excessively long experiment times. 

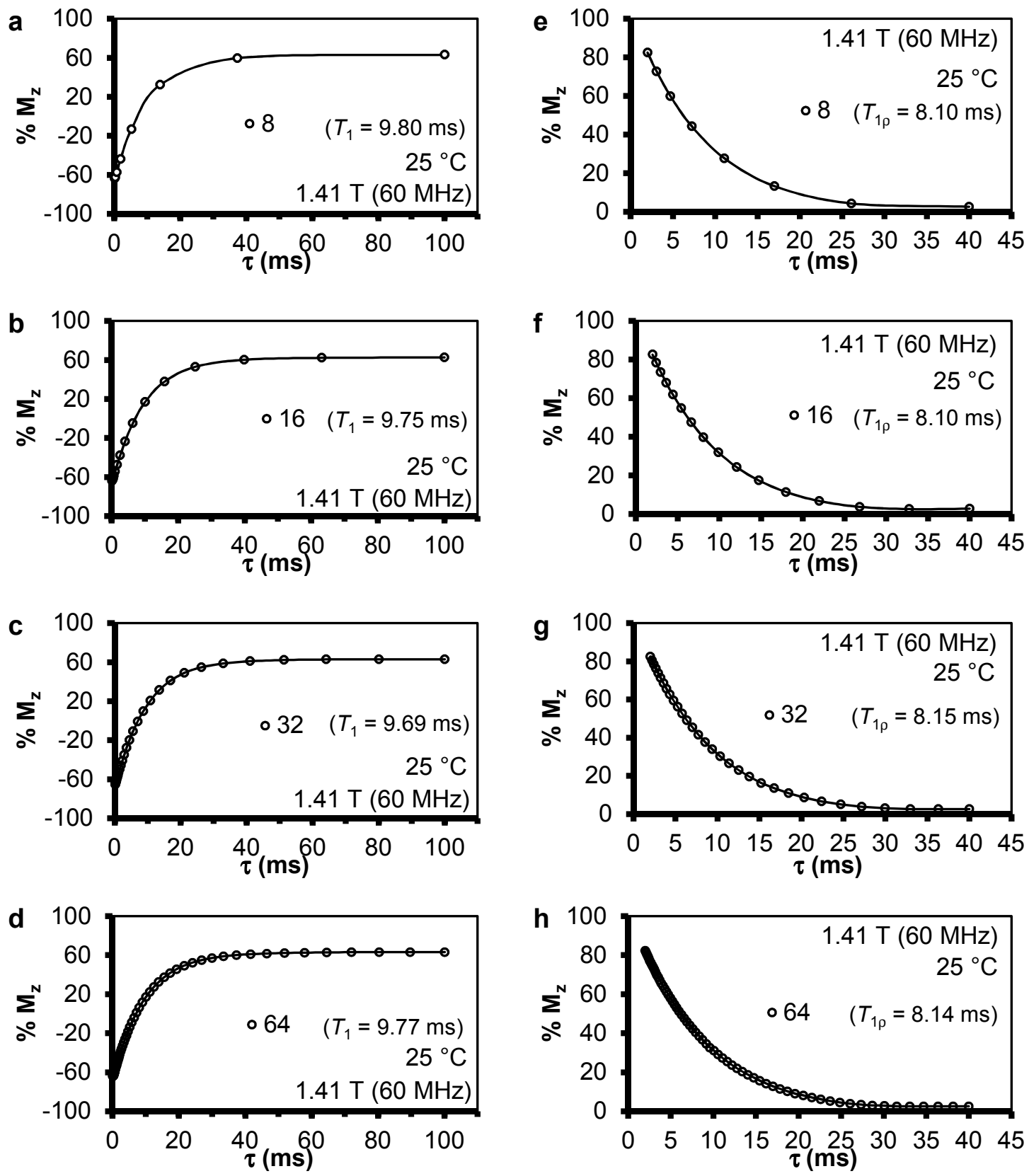

Figure 2.6: Effect of deliberate variations to the number of data points on $T_{1}(a-d)$ and $T_{1 p}(e-$ h) time curves. Sample: PBS pH 7.4 solution of Dotarem, $\left[\mathrm{Gd}^{3+}\right]=25 \mathrm{mM} . \mathrm{n}=1$. 
Table 2.6: Recommended pulse sequence parameter values for determinations of $T_{1}$ and $T_{1 \rho}$.

\begin{tabular}{|c|c|c|}
\hline Pulse sequence parameter & Inversion recovery & Spin locking \\
\hline Relaxation delay time (s) & $=10 \times T_{1}$ & $\begin{array}{c}=10 \times T_{1} \\
\text { or minimum } \\
\text { allowable } \geq 10 \times T_{1}\end{array}$ \\
\hline \multicolumn{3}{|l|}{ Time delays } \\
\hline First pulse (ms) & $<1 / 10$ th $\times T_{1}$ & $>1 / 10$ th $\times T_{1}$ \\
\hline Last pulse (ms) & $>5 \times T_{1}$ & $\begin{array}{c}>5 \times T_{1} \\
\text { or maximum allowed } \\
(40 \mathrm{~ms})\end{array}$ \\
\hline $\begin{array}{l}\text { Number of pulse delays for mono- } \\
\text { exponential behavior }\end{array}$ & 16 & 16 \\
\hline
\end{tabular}

\subsubsection{Pulse delay times}

These results demonstrate that significant variation in time constant values (standard deviation greater than the standard deviation due to natural variation in the data) were observed when different proportions of the relaxation time curve are measured by varying the first and last pulse delay times. Additionally, scatter of data points is possible with pulse delay times that are too short. First and last pulse delay times are sample specific parameters and therefore exact values are not recommended. However, it is recommended that time constants be generated by measuring as much of the time curve as possible that allows scatter to be avoided. Therefore, first and last pulse delay times of $<1 / 10^{\text {th }} \times T_{1}$ and $>5 \times T_{1}$ respectively are recommended when determining $T_{1}$ values with the inversion recovery pulse sequence and $>1 / 10^{\text {th }} \times T_{1}$ and $>5 \times T_{1}$ respectively when determining $T_{1 \rho}$ values with the spin lock pulse sequence. It should be noted that a 
last pulse delay $>5 \times T_{1}$ is not always possible due to instrument limitations to prevent hardware failure. In this case the maximum allowable should be employed. Reliable time constants may be generated with longer first pulse and shorter last pulse delay times, however, the recommended values allow for as much of the time curve as possible to be measured.

\subsubsection{Number of data points}

It has been demonstrated that time constants were not affected significantly (standard deviation was less than the standard deviation due to natural variation in the data) by the number of data points ranging from between $8-64$. However, the recommended number is 16 as this provides more data around the inflection of the time curve whilst keeping instrument and operator time to a minimum.

\subsection{Conclusions}

When deliberate variations were made to the suggested "ideal" pulse sequence parameter values for the three parameters investigated good fittings were generally still observed and time constants within did not vary by more than the natural variation in the data. The exception was when an insufficient proportion of the time curve was measured by varying the first and last pulse delay times. This suggests that this parameter is most critical for obtaining reliable time constants, more so than the number of data points along the time curve and the relaxation delay time employed. By investigating the effect the parameter values have on time constants the values in Table 2.6 are recommended. 
These should provide accurate time constants that do not vary by more than the natural variation in the data and ensure confidence in NMRD profiles generated from them. 


\section{CHAPTER 3. EFFECT OF SPIN LOCK FIELD STRENGTH $\left(\beta_{1}\right)$ ON THE ROTATING FRAME LONGITUDINAL RELAXATION RATE CONSTANT $\left(R_{1 \rho}\right)$}

\subsection{Establishing the $\mathcal{B B}_{1}$ independency of $R_{1 \rho}$}

Jordan and co-workers ${ }^{70}$ wrote a comprehensive paper describing the effect of a hydrated paramagnetic ion, undergoing solvent exchange, would have on the rotating frame longitudinal relaxation rate constant $\left(R_{1 \rho}\right)$ of bulk solvent nuclei (Equation 3.1), in which $R_{2 \mathrm{~W}}$ is the laboratory frame transverse relaxation rate constant of the solvent water; $q$ the number of water molecules bound to the paramagnetic ion; $[\mathrm{M}]$ the concentration of the metal ion, $\tau_{\mathrm{M}}$ the average water residence lifetime of a coordinated water molecule; $R_{1 \mathrm{M}}$ and $R_{2 \mathrm{M}}$ are the laboratory frame longitudinal and transverse relaxation rate constants of the coordinated water, respectively; $\Delta \omega_{M}$ is the difference in Larmor frequency between coordinated and solvent water protons; and $\omega_{1}$ is the Rabi frequency of the spin lock pulse). The magnitude of the spin lock field $\left(B_{1}\right)$ is given by Equation 3.2.

$$
\begin{gathered}
R_{1 \rho}=R_{2 W}+\frac{q[M]}{55.6} \times \frac{1}{\tau_{M}}\left(\frac{R_{2 M}}{R_{2 M}{ }^{1} / \tau_{M}}\right)\left\{\frac{\left(R_{1 M}+1 / \tau_{M}\right)\left(R_{2 M}+1 / \tau_{M}+\frac{\Delta \omega_{M}^{2}}{R_{2 M}}\right)+\omega_{1}^{2}}{\left(R_{1 M}{ }^{1} / \tau_{M}\right)\left(R_{2 M}{ }^{1} / \tau_{M}+\frac{\Delta \omega_{M}^{2}}{R_{2} M^{+1} / \tau_{M}}\right)+\omega_{1}^{2}}\right\} \\
\omega_{1}=-\gamma B_{1}
\end{gathered}
$$

Due to the isotropic $\mathrm{f}$ shell electronic configuration of the $\mathrm{Gd}^{3+}$ ion it does not induce a hyperfine shift. This means that $\Delta \omega_{M}^{2}$ will be close to zero, at the same time the high 
paramagnetism of $\mathrm{Gd}^{3+}$ means that $R_{2 \mathrm{M}}$ will be very large. This invokes the following condition identified by Jordan and co-workers.

$$
R_{2 M} \gg \Delta \omega_{M}^{2}
$$

In the presence of $\mathrm{Gd}^{3+}$ ions this condition is met and Equation 3.1 simplifies to;

$$
R_{1 \rho}=R_{2 W}+\frac{q[G d]}{55.6}\left(\frac{R_{2 M} \times 1 / \tau_{M}}{R_{1 M}{ }^{1} / \tau_{M}}\right)
$$

If Equation 3.4 is correct then there should be no dependence of $R_{1 \rho}$ on the spin lock field strength characterized in the above equations by the Rabi frequency $\left(\omega_{1}\right)$.

Expressed as rotating frame longitudinal relaxivity $\left(r_{1 \mathrm{r}}\right)$, Equation 3.4 becomes Equation 3.5 .

$$
r_{1 \rho}=\frac{q}{55.6}\left(\frac{R_{2 M} \times 1 / \tau_{M}}{R_{1 M}+1 / \tau_{M}}\right)
$$

To verify this theory, at low field ( 0.47 and $1.41 \mathrm{~T}, 20$ and $60 \mathrm{MHz}$ respectively) the rotating frame relaxation time constants were measured and plotted as the rate constant against the spin lock field strength $\left(\gamma B_{1}\right)$ for $100 \mathrm{mM}$ solutions of ProHance (cyclic) and Magnevist (linear) in PBS at 25 and $37{ }^{\circ} \mathrm{C}$. Due to longer SL pulses that can be played out by more powerful superconducting instruments, high field experiments (14.1 T, 600 $\mathrm{MHz})$ were performed with a less concentrated $(5 \mathrm{mM})$ solution of Dotarem (cyclic) in PBS at $25^{\circ} \mathrm{C}$. 


\subsection{Modulation of the strength of the spin lock field $\left(\mathcal{\gamma} B_{1}\right)$}

The flip angle $(\theta)$ of a RF pulse, in this case the spin lock pulse, is proportional to its strength $\left(\gamma B_{1}\right)$ and duration $(\tau)$ as shown in Equation 3.6.

$$
\theta=\gamma B_{1} \tau
$$

To achieve a pulse angle of $90^{\circ}$ the Bruker minispec instrument requires a pulse of duration $2.26 \mu \mathrm{s}$. From Equation 3.6 the strength of this pulse, expressed as frequency with units of $\mathrm{kHz}$, can be determined as follows;

$$
\begin{gathered}
\frac{\pi}{2} \times \frac{1}{2 \pi}=\frac{\gamma B_{1}}{2 \pi} \times 2.26(\mu \mathrm{s}) \\
\text { Given that } \frac{\gamma B_{1}}{2 \pi}=v_{1}(\mathrm{~Hz}) \\
\therefore \frac{1000}{4}=v_{1}(\mathrm{kHz}) \times 2.26(\mu \mathrm{s}) \\
\frac{250}{2.26(\mu \mathrm{s})}=v_{1}(\mathrm{kHz})=110.6 \mathrm{kHz}
\end{gathered}
$$

Although correctly calculated, this strength, $110.6 \mathrm{kHz}$, seems high. This value represents a large pulse power for the modest minispec instrument to generate. It is worth noting that the much more robust $600 \mathrm{MHz}$ spectrometer is set up to prevent the use of pulse powers in excess of $35 \mathrm{kHz}$. A resolution with the minispec manufacturer is ongoing at the time of writing and for the present purposes this value shall be used. 
The strength of the spin lock field was modulated by attenuating its power. Measured in decibels $(-\mathrm{dB})$, power attenuation describes the relationship between two power quantity values where as shown in Equation 3.7 where; $\mathrm{P}_{\text {in }}$ is input power, $\mathrm{P}_{\text {out }}$ is attenuated power and the factor of 10 accounts for a $\mathrm{dB}$ being $1 / 10^{\text {th }}$ of a bel.

$$
\text { Attenuation }(-d B)=10 \log _{10}\left(\frac{P_{\text {out }}}{P_{\text {in }}}\right)
$$

However, a modified attenuation equation must be used for field quantities, such as field strength, since the field is generated by current whose square is proportional to power as shown;

$$
\begin{gathered}
P=V \times I \\
\text { from Ohm's law; } V=I \times R \\
P=(I \times R) \times I=I^{2} \times R \\
P \propto I^{2}
\end{gathered}
$$

By substituting the power ratio for a field quantity such as $\gamma B_{1}$, the modified attenuation equation can be derived;

$$
\begin{aligned}
& \text { Attenuation }(-d B)=10 \log _{10}\left(\frac{\left(\gamma B_{1}^{\text {out }}\right)^{2}}{\left(\gamma B_{1}^{\text {in }}\right)^{2}}\right) \\
& \text { Attenuation }(-d B)=10 \log _{10}\left(\frac{\gamma B_{1}^{\text {out }}}{\gamma B_{1}^{\text {in }}}\right)^{2} \\
& \text { Attenuation }(-d B)=20 \log _{10}\left(\frac{\gamma B_{1}^{\text {out }}}{\gamma B_{1}^{\text {in }}}\right)
\end{aligned}
$$


Unless otherwise stated for our experiments we attenuated the power in $1 \mathrm{~dB}$ increments between $-40--6 \mathrm{~dB}$, the maximum attenuation permitted by the low fixed field relaxometer instruments. The Rabi frequencies that characterize the strength of the spin lock field over this attenuation range correspond to $1.1-55.4 \mathrm{kHz}$.

\subsection{Effect of $\not B_{1}$ on $R_{1 \rho}$ at low $B_{0}$ field strengths}

All profiles show the same basic features regardless of temperature, field or chelate structure. From Figure 3.1 it can clearly be seen for ProHance at $0.47 \mathrm{~T}(20 \mathrm{MHz})$, which represents agents based on the cyclic ligand DOTA, that when the strength of the spin lock field is $<10 \mathrm{kHz}$ the mean $R_{1 \rho}$ values are scattered and the standard deviations of the six measurements are large (Figure 3.1a). As the strength of the spin lock field increases $\left(\gamma B_{1}>10 \mathrm{kHz}\right)$ the mean $R_{1 \rho}$ values are more reproducible with lower standard deviations and essentially independent of $\gamma B_{1}$. From the same plot, it is also apparent that by increasing the temperature from $25{ }^{\circ} \mathrm{C}$ (open circles) to $37{ }^{\circ} \mathrm{C}$ (closed circles) the rotating frame relaxation rates decrease slightly, a consequence of ProHance, like all other clinical agents studied herein, being in the fast exchange regime. An increase in temperature is expected to result in an increase in $T_{1 \rho}$, as for $T_{1}$, due to a shortening of the correlation time $\left(\tau_{\mathrm{c}}\right)$ and less efficient dipole-dipole interactions. On moving to $1.41 \mathrm{~T}$ (60 MHz) comparable observations were made (Figure 3.1b). As was the case for Magnevist, an agent based on the linear ligand DTPA (Figure 3.1c and d at 0.47 and 1.41 T respectively). 


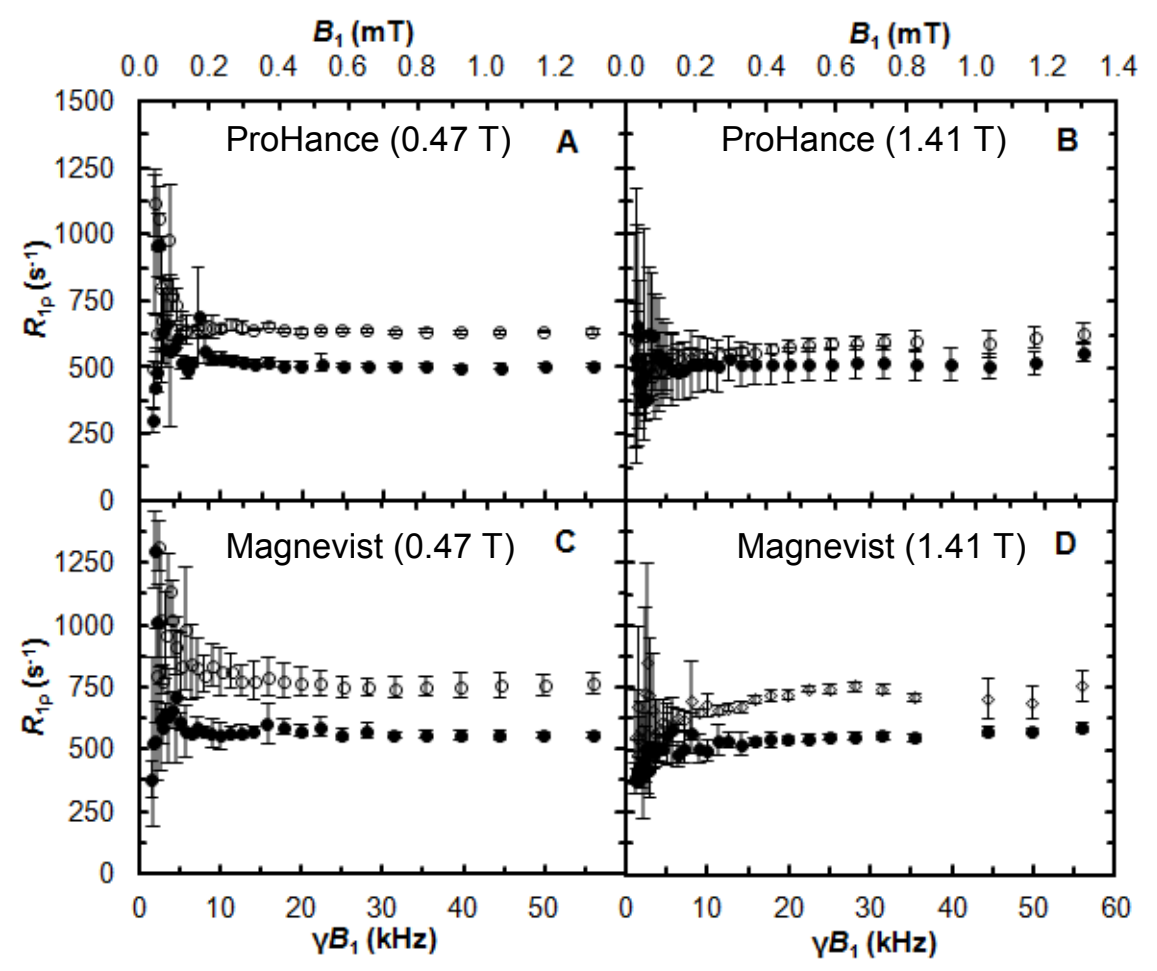

Figure 3.1: The dependence of the water proton rotating frame longitudinal relaxation rate constant $\left(R_{1 \mathrm{p}}\right)$ on the strength of the spin lock field $\left(\gamma B_{1}\right)$ for a single PBS pH 7.4 solution of each contrast agent with a [Gd $\left.\mathrm{Gd}^{3+}\right]$ of $100 \mathrm{mM}$ at $25^{\circ} \mathrm{C}$ (open symbols) and $37^{\circ} \mathrm{C}$ (closed symbols). ProHance, 0.47 T (a), 1.41 T (b). Magnevist, 0.47 T (c), $1.41 \mathrm{~T}(\mathrm{~d})$. Data shown are the mean of six measurements; the error bars represent the SD of the data. Note: $\gamma B_{1}$ values calculated from a $90^{\circ}$ pulse duration reported from the minispec instrument. These values are surprisingly large and suggest the the pulse duration reported is incorrect.

It follows from these experiments that in order to generate accurate and precise $R_{1 \rho}$ data, useful for creating NMRD profiles, effective spin locking must occur, rendering $R_{1 \rho}$ independent of $\gamma B_{1}$. From this data this appears to occur with a minimum spin locking field strength $\left(\gamma B_{1}\right)$ of $\sim 10 \mathrm{kHz}$, however, this value is unrealistic and we demonstrate in the next section that it is possible to spin lock with a pulse of a few hundred $\mathrm{Hz}$. 
A limitation of the experimental design was a lack of randomization. For each agent, temperature and $\mathrm{B}_{0}$ field strength the time constants were measured sequentially, starting at high powers and working towards lower powers. Moreover, the software measures signal intensity as a function of increasing pulse delay times rather than sampling the pulse delay times randomly. Randomization to reduce bias from other experimental factors would add a higher degree of validity to the claims here that the rotating frame longitudinal relaxation rate constant is independent of the strength of the spin lock pulse. The raw time curve data is not routinely saved by the low field minispec instrument software used for these experiments and therefore could not be shown. As a result, it was not available for offline processing. This could have provided a means to rule out any biexponential or multiexponential behavior as a source of contribution to the observed independence of $R_{1 \rho}$ on $\gamma B_{1}$.

\subsection{Effect of $\not B_{1}$ on $R_{1 \rho}$ at high $B_{0}$ field strengths}

Our high field (14.1 T, $600 \mathrm{MHz}) \gamma B_{1}$ dependency plot (Figure 3.2) shows similar facets to those observed at low $B_{0}$ fields (Figure 3.1). Namely, the rotating frame longitudinal relaxation rate $\left(R_{1 \rho}\right)$ of a $5 \mathrm{mM}$ solution of Dotarem (cyclic-type agent) studied shows greater scatter at low spin lock field strengths. This again is reduced, as is the error associated with the data, as the strength of the spin lock pulse is increased. It is, however, noteworthy to point out some differences from the equivalent low field experiments. The strength of the spin lock pulse required to effectively lock the spins at high $B_{0}$ field is far less, and much more realistic, than those determined from our low 
field experiments, with $R_{1 \rho}$ values stabilizing at about $500 \mathrm{~Hz}$. Additionally, the $R_{1 \rho}$ values, and their associated errors, are significantly reduced at $14.1 \mathrm{~T}(600 \mathrm{MHz})$. Lastly, as a result of the increased precision and reduced errors in our high field data a notable trend was observed in the high field data. From the point the $R_{1 \rho}$ data becomes stable to the end of the range of field strengths studied $(\sim 500-20000 \mathrm{~Hz})$ the $R_{1 \rho}$ values decrease by approximately $5 \%$. Although this is not an expected trend we believe the observation is not due to any limitation in the theory and offer two possible explanations for which both may contribute to this observation. Firstly, with the relatively long spin lock pulses (relative to those required at low field with higher $\mathrm{Gd}^{3+}$ concentrations) required to observe full signal decay and the incremental decrease in attenuation, buildup of heat from many successive experiments could be responsible for the observation. In unrelated experiments (not reported) significant increases in sample temperature were observed when long spin lock pulses were being employed. Secondly, at high $B_{0}$ field strengths the reduction of the effective field by the application of a spin lock pulse may be limiting. As a result, as the spin lock field strength increases the Rabi frequency may approach the Larmor frequencies in which dispersion of $R_{1}$ occurs due to $R_{1 \mathrm{M}}$ and $R_{2 \mathrm{M}}$ dependencies giving rise to the observed decreases in $r_{1}$.

The reduced spin lock field strength required to effectively lock the spins at high $B_{0}$ fields compared to low $B_{0}$ fields as a consequence of two factors. First, the magnetic field homogeneity of the low field instruments is less than that of the high field superconducting spectrometers used in this study since the low field instruments are 
absent of shim stacks capable of tuning the field homogeneity. Accordingly, the line width of the water proton resonance will be greater at low $B_{0}$ field strength. Secondly, due to other limitations of the low field instruments the length of the maximum spin lock pulse duration capable of being employed is substantially shorter than that achievable with the high field instruments. As a result, higher agent concentration was necessary and this also contributes to the increased line width of the signal.

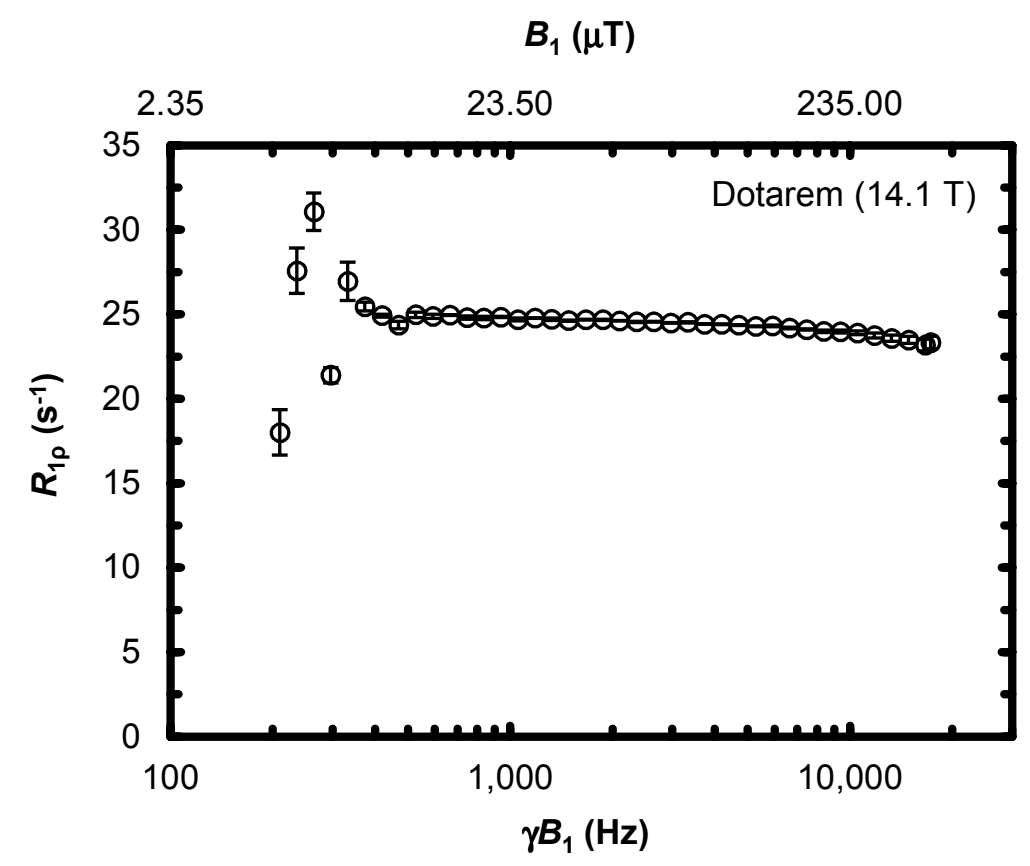

Figure 3.2: The dependence of the water proton rotating frame longitudinal relaxation rate constant $\left(R_{1 \mathrm{p}}\right)$ on the spin lock field streng th $\left(\gamma B_{1}\right)$ for a single PBS $\mathrm{pH} 7.4$ solution of Dotarem with a $\left[\mathrm{Gd}^{3+}\right]$ of $5 \mathrm{mM}$ at $14.1 \mathrm{~T}(600 \mathrm{MHz})$ and $25^{\circ} \mathrm{C}$. Data shown are the mean of six measurements; the error bars represent the SD of the data.

As for our low $B_{0}$ field strength study, our high $B_{0}$ field strength study was conducted by measuring rotating frame relaxation time constants and plotting the rate constant as a 
function of the spin lock field strength $\left(\gamma B_{1}\right)$. However, unlike the low field instruments, the high resolution instrument allows for a Fourier transformed spectrum to be obtained as described in section 1.6.2. Figure 3.3 shows raw data, sampled from the sixth repetition of the analysis of the Dotarem sample, for a select number of spin lock field strength values $\left(\gamma B_{1}\right)$ used to generate Figure 3.2. The frequency domain spectrum shown for each $\gamma B_{1}$, corresponds to the FID produced from the acquisition of the pulse sequence with the shortest time delay in the experiment. The complete time curve is shown as the overlay along with the respective time and rate constant. This figure demonstrates that the time curves, generated from the decay of the signal intensity of the spectrum with subsequent delay times, allow for full decay of the signal to be observed and that the observed data is well fitted by Equation 1.24 thus providing reliability of the data in Figure 3.2.

\subsection{A comparison between spin locking and CPMG with short pulse delay times}

The CPMG pulse sequence for determining laboratory frame transverse relaxation time constants $\left(T_{2}\right)$ can be likened to a spin lock pulse sequence if the variable pulse delay $(\tau)$, the delay time between the $180^{\circ}$ pulses of the echo train, is very short. In such a situation the $180^{\circ}$ refocusing pulses can be considered to be permanently on. The difference, however, being that the $180^{\circ}$ refocusing pulse of the CPMG sequence is a 'hard' pulse (high power and short duration) that undergoes many phase changes whereas the spin lock pulse is 'soft' (low power long duration) and does not change phase whilst employed. 

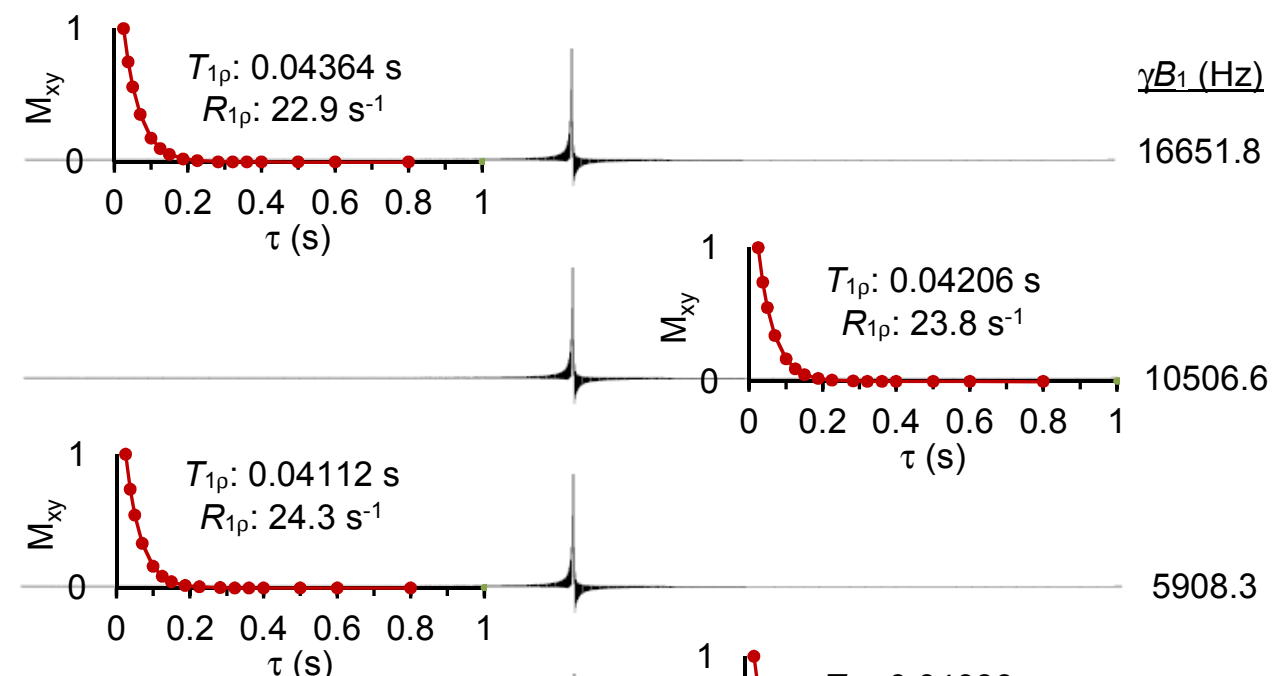

5908.3

3322.5

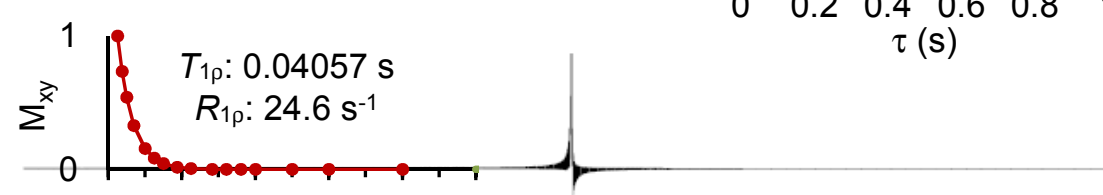

1050.7

1868.4
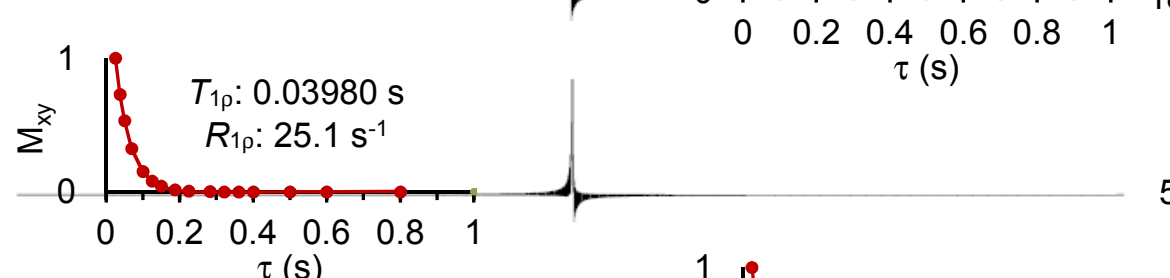

590.8

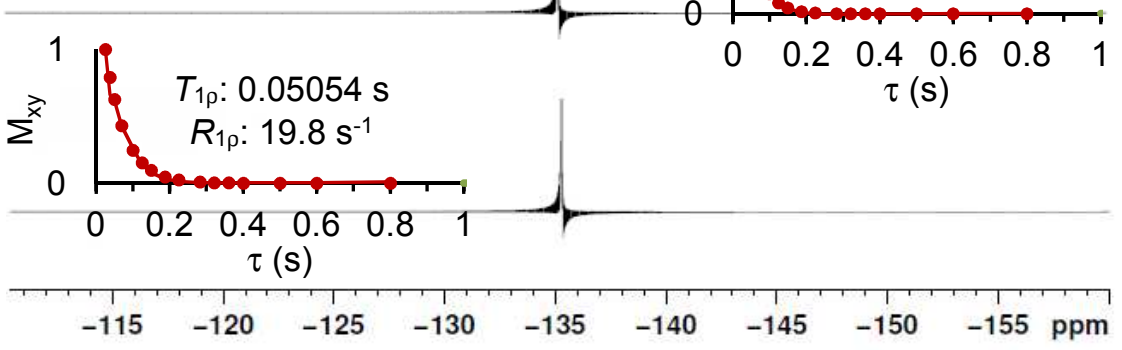

209.6

Figure 3.3: Sample raw data for the $n=6$ determination of $R_{1 p}$ for several pulse strengths used to prepare Figure 3.2. Spectra were obtained from the FT of the FID from the acquisition with shortest time delay $(\tau)$. Time $\left(T_{1 \rho}\right)$ and rate $\left(R_{1 \rho}\right)$ constants are also shown. 
Given their similarity it was important to compare time constants generated from relaxation data obtained from both pulse sequences. The same PBS solution of Dotarem prepared at a $\mathrm{Gd}^{3+}$ concentration of $25 \mathrm{mM}$ used for the experiments described in Chapter 2 was used. We limited investigations to our $20 \mathrm{MHz}(0.47 \mathrm{~T})$ fixed low field relaxometer and measurements were only made at the historically relevant temperature of $25{ }^{\circ} \mathrm{C}$. For this experiment we measured $T_{2}$ with a series of different pulse delay times keeping the total experiment time constant by varying the number of data points. It was only possible to investigate a limited range of $\tau$ values for the CPMG pulse sequence, due to a minimum limit of $40 \mathrm{~ms}$. The time constants were plotted as a function of the variable pulse delay time $(\tau)$ and compared to the rotating frame longitudinal relaxation time constant for the same sample (Figure 3.4). Pulse powers for all measurements were set to $6 \mathrm{~dB}$ for continuity.

We have demonstrated (Figure 3.4) that over the range of pulse delay times investigated $T_{2}$ did not tend to $T_{1 \rho}$ as $\tau$ varied. In fact, there was no significant change in the $T_{2}$ value at all. The standard deviation of mean $T_{2}$ values $(0.05 \mathrm{~ms})$ was less than the natural variation of the data for the similar time constant, $T_{1 \rho}$, at $25^{\circ} \mathrm{C}$ on the same instrument determined in chapter 2 (Table 3.1). This suggests that a CPMG pulse sequence with short pulse delay times is not equivalent to a spin locking pulse sequence, a likely consequence of the CPMG pulse sequence employing 180 refocusing pulse with alternating phase which does not result in spin locking. 


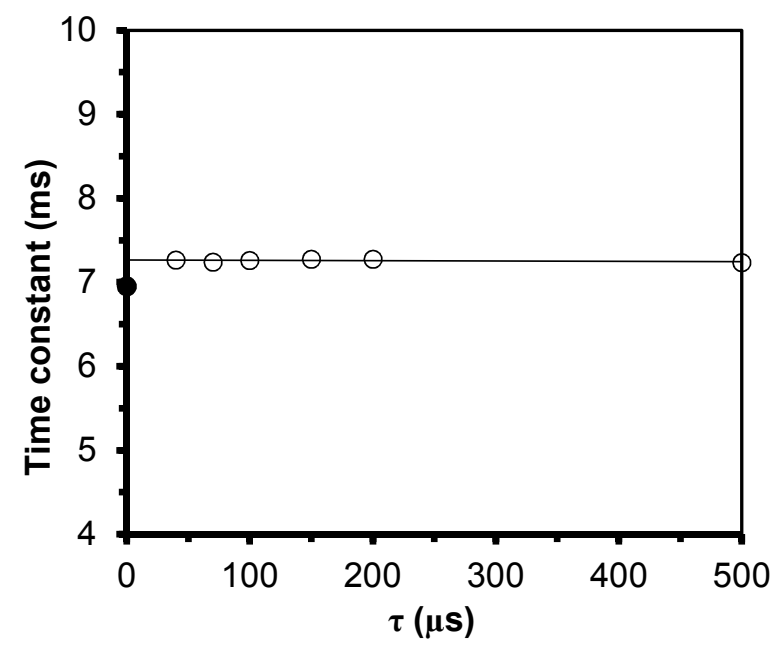

Figure 3.4: The dependence of the laboratory frame transverse relaxation time constant $\left(T_{2}\right)$ on the variable pulse delay time $(\tau)$ for a single PBS $\mathrm{pH} 7.4$ solution of Dotarem with a $\left[\mathrm{Gd}^{3+}\right]$ of 25 $\mathrm{mM}$ at $0.47 \mathrm{~T}(20 \mathrm{MHz})$ and $25^{\circ} \mathrm{C}$. The filled circle is the respective rotating frame longitudinal relaxation time constant $\left(T_{1 \rho}\right)$. Data shown are the mean of three measurements. Error bars representing the SD of the data were omitted for clarity but all were smaller than the markers.

The line throught the $T_{2}$ data is to guide the eye only.

Table 3.1: Effect of the pulse delay time $(\tau)$ on mean $T_{2}$ values $(n=3)$ at $0.47 \mathrm{~T}(20 \mathrm{MHz})$.

\begin{tabular}{c|c} 
& $T_{2}(\mathrm{~ms})$ \\
\hline Pulse delay time $(\mu \mathrm{s})$ & $\mathbf{2 5}{ }^{\circ} \mathrm{C}$ \\
\hline $\mathbf{4 0}$ & 7.26 \\
$\mathbf{1 0 0}$ & 7.24 \\
$\mathbf{1 5 0}$ & 7.26 \\
$\mathbf{2 0 0}$ & 7.28 \\
$\mathbf{5 0 0}$ & 7.27 \\
\hline Pulse delay time $S D$ & 7.23 \\
\hline Natural variance $S D\left(T_{1 \rho}\right)$ & 0.018 \\
\hline
\end{tabular}




\subsection{Conclusions}

The rotating frame relaxivity of $\mathrm{Gd}^{3+}$-based CAs have been shown to be essentially independent of the magnitude of the $B_{1}$ field used to effect spin locking. This is consistent with previously reported theory as predicted by Jordan and co-workers. ${ }^{70}$ This result is important because it allows the value of $\gamma B_{1}$ to be neglected when comparing rotating frame relaxivities of $\mathrm{Gd}^{3+}$-based CAs across several $B_{0}$ fields. Moreover it seems that a CPMG pulse sequence with short variable pulse delay times and a spin locking pulse sequences do not measure the same relaxation mechanism. 
CHAPTER 4. A COMPARISON OF LABORATORY (ri) AND ROTATING (rip) FRAME LONGITUDINAL RELAXIVITIES FOR $q=1$ CLINICAL Gd ${ }^{3+}$-BASED CAs

\subsection{Establishing the potential benefits of spin locking on relaxivity}

Although well described by theory, the laboratory frame relaxivities of clinical $\mathrm{Gd}^{3+}$ based CAs are low, typically $4-5 \mathrm{mM}^{-1} \mathrm{~s}^{-1}$ (Figure 1.15 ). The low relaxivities arise from the rapid molecular tumbling of these low molecular weight chelates (short $\tau_{\mathrm{R}}$ ) and practical restrictions on their hydration number (q). These low relaxivities result in high detection limits for these agents, necessitating high doses. Agents with bulky substituents have longer $\tau_{\mathrm{R}}$ values, a result of slower tumbling, which increases relaxivity. However, relaxivity often continues to be limited by water exchange parameters and relaxivity invariably falls short of the theoretically calculated maximum. Slowly tumbling chelates with more rapid water exchange rates $\left(1 / \tau_{\mathrm{M}}\right)$ afford the highest relaxivities, at current clinical imaging fields. However, clinical imaging is being performed at increasingly higher magnetic field strengths and the optimal $\tau_{M}$ value for $r_{1}$ relaxivity is found to decrease as $B_{0}$ increases. $^{30}$ Furthermore, we have recently shown that the relationship between water exchange, molecular tumbling and relaxivity in $\mathrm{Gd}^{3+}$ chelates is more complex than previously appreciated, ${ }^{74}$ as a result the theoretical relaxivity potential may be somewhat lower than previously thought. Improvements in detectability could also be made by increasing the hydration number $(q)$ of the complex. Through opening up additional coordination sites to water relaxivity increases, however, this is at the cost of 
reduced chelate stability. Even if a new "gold standard" agent were to be designed that could afford high relaxivities at high fields a lengthy and costly approvals process would ensue.

It has been conceived elsewhere ${ }^{75-80}$ that more significant gains in agent detectability may be possible by imaging at lower effective $B_{0}$ fields where relaxivity is likely to be greater. One method to produce such an image is to generate contrast based on the rotating frame longitudinal relaxation time constant $\left(T_{1 \rho}\right) . \quad T_{1 \rho}$ contrast is generated by employing a weak spin locking pulse parallel to the net magnetization in the $\mathrm{x}, \mathrm{y}$-plane and measuring the decay of transverse magnetization in the rotating frame of reference as a function of the spin locking time. Time constants generated in this manner are expected to be characteristic of those measured at low fields. If proven successful this technique would have the added benefit of a relatively quick adoption in to clinical practice. Moreover, if performed in the presence of a large external static $B_{0}$ field this would allow the $\mathrm{s} / \mathrm{n}$ advantages of these fields to be maintained. ${ }^{81}$

At the time of writing very few reports pertaining to this hypothesis exist. ${ }^{82-85}$ With the exception of one publication investigating spin locking with Ablavar, ${ }^{82}$ the focus has solely been on Magnevist and the study of imaging metrics such as contrast and contrast to noise ratio $(\mathrm{CNR})^{83-85}$ rather than the specific effect on relaxivity, a key indicator of CA effectiveness and determinant of agent detectability.

Accordingly, we set out to conduct a thorough investigation into the effect of spin locking on the relaxivity of seven $\mathrm{q}=1$ clinical $\mathrm{Gd}^{3+}$-based CAs by generating and 
comparing, under identical conditions, $r_{1}$ and $r_{1} \rho$ relaxivities as a function of $B_{0}$ in the form of NMRD profiles. Due to structural similarities of OptiMARK and Eovist/Primovist to Ominscan and MultiHance respectively, these agents were not studied. NMRD profiles for the seven agents were grouped according to the three structural categories identified in Table 1.1; linear, macrocyclic and bulky. Samples of the agents were prepared in phosphate buffered saline $\mathrm{pH} 7.4$ and relaxivities determined over a range of $B_{0}$ field strengths, $0.47-14.1 \mathrm{~T}(20-600 \mathrm{MHz}$ respectively), at both 25 and $37^{\circ} \mathrm{C}$.

\subsection{Comparison of $r_{1}$ and $r_{1 \rho}$ for linear and macrocyclic $q=1$ clinical Gd $^{3+}$-based $C A s$}

With the exception of Gadavist, whose relaxivities are slightly elevated over the others, there is little observable difference between the NMRD profiles generated for linear and macrocyclic agents other than the specific relaxivities measured (Figure 4.1). This demonstrates that there is little effect on both $r_{1}$ and $r_{1 \rho}$ at these fields as a result of the known differences in electron spin relaxation $\left(T_{1 \mathrm{e}} / T_{2 \mathrm{e}}\right)$ for both macrocyclic and linear chelates. ${ }^{86}$ However, for all chelates of these two classes, $r_{1 \rho}$ values are higher than $r_{1}$ values across their entire NMRD profile and both decrease as $B_{0}$ increases. It is also noticed that $r_{1 \rho}$ falls of slightly less rapidly than $r_{1}$ does. This shows that by spin locking it is possible to "recover" relaxivity lost by moving to higher fields for these agents. However, the gains are fairly modest showing an increase in relaxivity by a factor of just 1.22 at $0.47 \mathrm{~T}$ on average, rising to 1.33 at $14.1 \mathrm{~T}$ on average (Table $4.1-4.4$ ). 


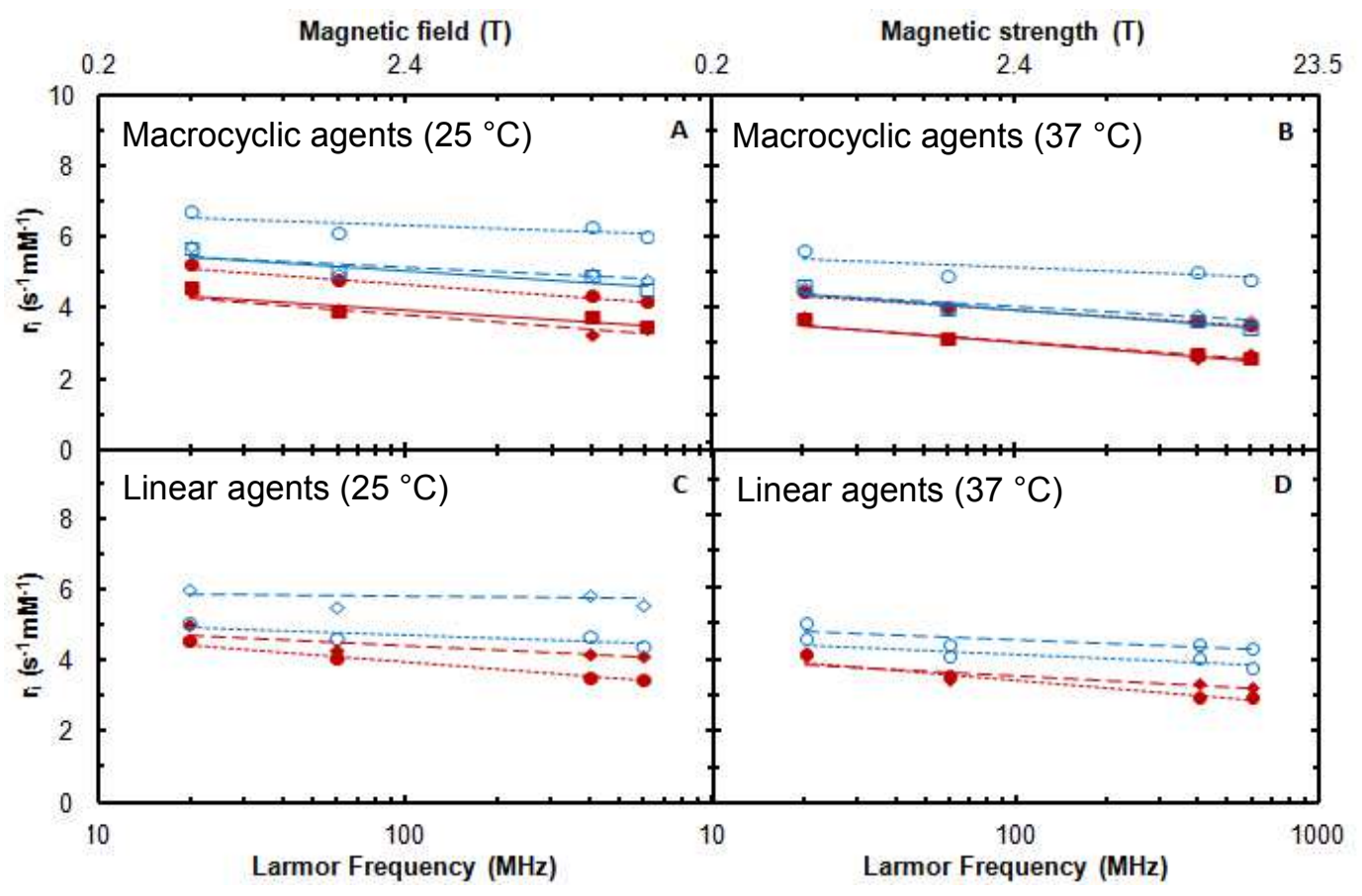

Figure 4.1: NMRD profiles of the linear and macrocyclic clinical contrast agents studied herein at $25^{\circ} \mathrm{C}(\mathrm{A} \& \mathrm{C})$ and $37^{\circ} \mathrm{C}(\mathrm{B} \& \mathrm{D})$ in PBS: laboratory frame data $\left(r_{1}\right)$ are presented in red (closed symbols); and rotating frame data $\left(r_{1 \rho}\right)$ are presented in blue (open symbols); $\omega_{1}=35 \mathrm{kHz}(0.47$ and $1.41 \mathrm{~T}$ ) and 6924.2 and $17237.0 \mathrm{~Hz}$ (9.4 and 14.1 T respectively). A \& B: macrocyclic agents: Dotarem (squares); ProHance (diamonds); Gadovist (circles). C \& D: linear agents: Magnevist (diamonds); Omniscan (circles). Lines through the data are to guide the eye only. Data shown are the mean of six measurements; error bars representing the SD of the data were omitted for clarity, all were smaller than the markers.

At both clinical $(0.47-1.41 \mathrm{~T})$ and research $(9.4-14.1 \mathrm{~T})$ field strengths, if a linear or macrocyclic agent is desired with high relaxivity then it is clear from Figure 4.1a and b that the agent of choice would be Gadavist and that a spin locking pulse should be applied. However, from Tables $4.1-4.4$ it is clear that although Gadavist has the highest relaxivity of all the linear and macrocyclic agents, with or without spin locking, 
Table 4.1: Raw $r_{1}$ and $r_{1 \rho}$ data at $25^{\circ} \mathrm{C}$ used to generate the NMRD above for linear and macrocyclic clinical agents studied herein at low field and their calculated ratios. Mean represents the average difference for the specified category of agents.

\begin{tabular}{|c|c|c|c|c|c|c|c|c|}
\hline & & & \multicolumn{2}{|c|}{$0.47 \mathrm{~T}$} & \multirow{2}{*}{$r_{1 \rho} / r_{1}$} & \multicolumn{2}{|c|}{$1.41 \mathrm{~T}$} & \multirow{2}{*}{$r_{1 \rho} / r_{1}$} \\
\hline & & & $r_{1}\left(\mathrm{mM}^{-1} \mathrm{~s}^{-1}\right)$ & $r_{1 \rho}\left(m^{-1} s^{-1}\right)$ & & $r_{1}\left(\mathrm{mM}^{-1} \mathrm{~s}^{-1}\right)$ & $r_{1 p}\left(\mathrm{mM}^{-1} \mathrm{~s}^{-1}\right)$ & \\
\hline \multirow{5}{*}{$25^{\circ} \mathrm{C}$} & \multirow{3}{*}{ Macrocyclic } & Dotarem & 4.60 & 5.72 & 1.24 & 3.96 & 5.07 & 1.28 \\
\hline & & ProHance & 4.53 & 5.79 & 1.28 & 4.01 & 5.01 & 1.25 \\
\hline & & Gadovist & 5.28 & 6.79 & 1.29 & 4.85 & 6.20 & 1.28 \\
\hline & \multirow{2}{*}{ Linear } & Magnevist & 5.06 & 6.07 & 1.20 & 4.33 & 5.59 & 1.29 \\
\hline & & Omniscan & 4.64 & 5.12 & 1.10 & 4.15 & 4.66 & 1.12 \\
\hline & & Mean & & & 1.22 & & & 1.24 \\
\hline
\end{tabular}


Table 4.2: Raw $r_{1}$ and $r_{1 \rho}$ data at $37^{\circ} \mathrm{C}$ used to generate the NMRD above for linear and macrocyclic clinical agents studied herein at low field and their calculated ratios. Mean represents the average difference for the specified category of agents.

\begin{tabular}{|c|c|c|c|c|c|c|c|c|}
\hline & & & \multicolumn{2}{|c|}{$0.47 \mathrm{~T}$} & \multirow{2}{*}{$r_{1 \rho} / r_{1}$} & \multicolumn{2}{|c|}{$1.41 \mathrm{~T}$} & \multirow[b]{2}{*}{$r_{1 \rho} / r_{1}$} \\
\hline & & & $r_{1}\left(\mathrm{mM}^{-1} \mathrm{~s}^{-1}\right)$ & $r_{1 \rho}\left(\mathrm{mM}^{-1} \mathrm{~s}^{-1}\right)$ & & $r_{1}\left(\mathrm{mM}^{-1} \mathrm{~s}^{-1}\right)$ & $r_{1 \rho}\left(\mathrm{mM}^{-1} \mathrm{~s}^{-1}\right)$ & \\
\hline \multirow{5}{*}{$37^{\circ} \mathrm{C}$} & \multirow{3}{*}{ Macrocyclic } & Dotarem & 3.74 & 4.68 & 1.25 & 3.18 & 4.04 & 1.27 \\
\hline & & ProHance & 3.79 & 4.63 & 1.22 & 3.20 & 4.03 & 1.26 \\
\hline & & Gadovist & 4.50 & 5.67 & 1.26 & 4.06 & 4.96 & 1.22 \\
\hline & \multirow{2}{*}{ Linear } & Magnevist & 4.18 & 5.05 & 1.21 & 3.47 & 4.47 & 1.29 \\
\hline & & Omniscan & 4.20 & 4.60 & 1.10 & 3.62 & 4.12 & 1.14 \\
\hline & & Mean & & & 1.21 & & & 1.24 \\
\hline
\end{tabular}


Table 4.3: Raw $r_{1}$ and $r_{1 \rho}$ data at $25^{\circ} \mathrm{C}$ used to generate the NMRD above for linear and macrocyclic clinical agents studied herein at high field and their calculated ratios. Mean represents the average difference for the specified category of agents

\begin{tabular}{|c|c|c|c|c|c|c|c|c|}
\hline & & & \multicolumn{2}{|c|}{$9.4 \mathrm{~T}$} & \multirow{2}{*}{$r_{1 \rho} / r_{1}$} & \multicolumn{2}{|c|}{$14.1 \mathrm{~T}$} & \multirow{2}{*}{$r_{1 \rho} / r_{1}$} \\
\hline & & & $r_{1}\left(\mathrm{mM}^{-1} \mathrm{~s}^{-1}\right)$ & $r_{1 \rho}\left(\mathrm{mM}^{-1} \mathrm{~s}^{-1}\right)$ & & $r_{1}\left(\mathrm{mM}^{-1} \mathrm{~s}^{-1}\right)$ & $r_{1 \rho}\left(\mathrm{mM}^{-1} \mathrm{~s}^{-1}\right)$ & \\
\hline \multirow{5}{*}{$25^{\circ} \mathrm{C}$} & \multirow{3}{*}{ Macrocyclic } & Dotarem & 3.79 & 4.95 & 1.31 & 3.49 & 4.56 & 1.26 \\
\hline & & ProHance & 3.31 & 5.04 & 1.52 & 3.48 & 4.83 & 1.39 \\
\hline & & Gadovist & 4.42 & 6.35 & 1.44 & 4.23 & 6.05 & 1.43 \\
\hline & \multirow{2}{*}{ Linear } & Magnevist & 4.21 & 5.91 & 1.40 & 4.18 & 5.65 & 1.35 \\
\hline & & Omniscan & 3.58 & 4.74 & 1.32 & 3.54 & 4.43 & 1.25 \\
\hline & & Mean & & & 1.40 & & & 1.34 \\
\hline
\end{tabular}


Table 4.4: Raw $r_{1}$ and $r_{1 \rho}$ data at $37^{\circ} \mathrm{C}$ used to generate the NMRD above for linear and macrocyclic clinical agents studied herein at high field and their calculated ratios. Mean represents the average difference for the specified category of agents.

\begin{tabular}{|c|c|c|c|c|c|c|c|c|}
\hline & & & \multicolumn{2}{|c|}{$9.4 \mathrm{~T}$} & \multirow{2}{*}{$r_{1 \rho} / r_{1}$} & \multicolumn{2}{|c|}{$14.1 \mathrm{~T}$} & \multirow{2}{*}{$r_{1 \rho} / r_{1}$} \\
\hline & & & $r_{1}\left(\mathrm{mM}^{-1} \mathrm{~s}^{-1}\right)$ & $r_{1 \rho}\left(m^{-1} s^{-1}\right)$ & & $r_{1}\left(\mathrm{mM}^{-1} \mathrm{~s}^{-1}\right)$ & $r_{1 p}\left(\mathrm{mM}^{-1} \mathrm{~s}^{-1}\right)$ & \\
\hline \multirow{5}{*}{$37^{\circ} \mathrm{C}$} & \multirow{3}{*}{ Macrocyclic } & Dotarem & 2.73 & 3.70 & 1.36 & 2.61 & 3.48 & 1.33 \\
\hline & & ProHance & 2.64 & 3.84 & 1.45 & 2.73 & 3.70 & 1.36 \\
\hline & & Gadovist & 3.66 & 5.08 & 1.39 & 3.57 & 4.86 & 1.36 \\
\hline & \multirow{2}{*}{ Linear } & Magnevist & 3.36 & 4.46 & 1.33 & 3.24 & 4.33 & 1.34 \\
\hline & & Omniscan & 3.02 & 4.07 & 1.35 & 3.03 & 3.80 & 1.25 \\
\hline & & Mean & & & 1.37 & & & 1.33 \\
\hline
\end{tabular}


the observed increase by applying a spin locking pulse is not necessarily proportional to the magnitude of the laboratory frame values. For example, at $25{ }^{\circ} \mathrm{C}$ and $9.4 \mathrm{~T}$ the relaxivity of ProHance increases from $3.31 \mathrm{mM}^{-1} \mathrm{~s}^{-1}$ to $5.04 \mathrm{mM}^{-1} \mathrm{~s}^{-1}$ (a factor of 1.52) whereas Magnevist increases from $4.21 \mathrm{mM}^{-1} \mathrm{~s}^{-1}$ to $5.91 \mathrm{mM}^{-1} \mathrm{~s}^{-1}$ (a factor of 1.40) and Gadovist increases from $4.42 \mathrm{mM}^{-1} \mathrm{~s}^{-1}$ to $6.35 \mathrm{mM}^{-1} \mathrm{~s}^{-1}$ (a factor of 1.44). From the values in Table 4.2, if Gadavist is selected for a high field imaging application, on average, the relaxivity using a spin locking pulse increases by a factor of 1.68 when compared to values obtained from other linear and macrocyclic agents without spin locking.

Values of $r_{1}$ and $r_{1 \rho}$ used in Figure 4.1 were obtained by measuring the laboratory and rotating frame relaxation time constants $\left(T_{\mathrm{i}}\right)$, where $\mathrm{i}=1$ or $1 \rho$, provided by fitting the raw time curve data, comparable to those shown in chapter 3 , to the relevant fitting equations. From these, relaxivity plots were generated by plotting the rate constant $\left(1 / \mathrm{T}_{\mathrm{i}}\right)$ as a function of $\left[\mathrm{Gd}^{3+}\right]$. Linear regression analysis of these plots affords the relaxivity as the slope, according to the linear form of Equation 1.17. A sample of typical relaxivity plots generated to determine relaxivities for Figure 4.1 are provided in Figure 4.2 and demonstrate excellent correlation coefficients typically $>0.999$.

\subsection{A comparison of $r_{1 \rho}$ for linear and macrocyclic chelates to simulated theory}

To determine if the data observed is a true reflection of theory the relevant parameters required to simulate rotating frame NMRD profiles are required to be identified. For this, further analysis of the work set out by Jordan and co-workers is required. 

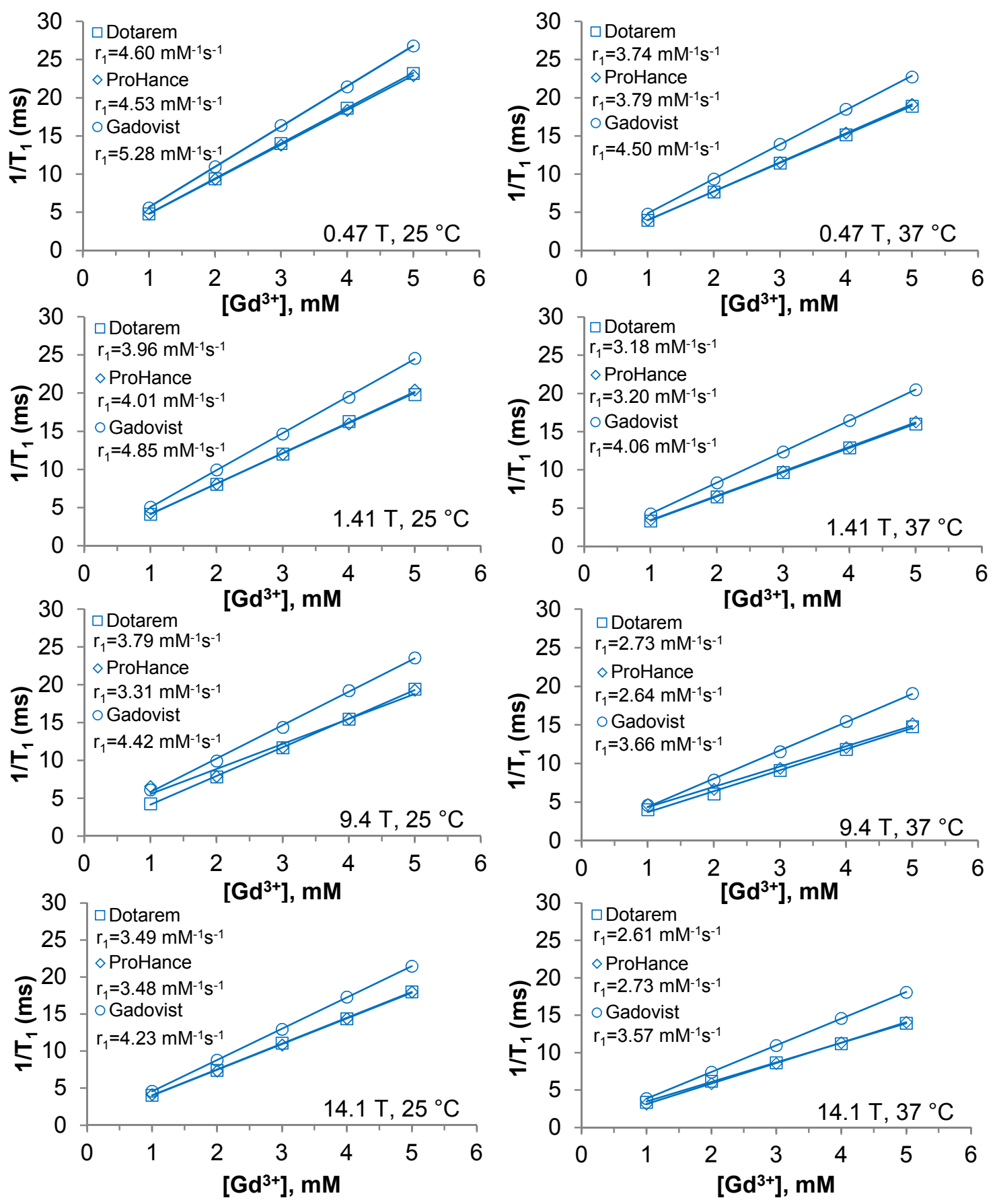

Figure 4.2: Sample relaxivity $\left(r_{1}\right)$ plots for the macrocyclic class of clinical agents. Data shown are the mean of six measurements with the mean relaxivity (slope) for each agent shown in the legend. $R^{2}$ for all agents are $>0.978$. Error bars representing the SD of the data were omitted for clarity, all were smaller than the markers. 
The dependence of $r_{1 \rho}$ in the presence of a $\mathrm{Gd}^{3+}$ ion was previously derived in Chapter 3 (Equation 3.5) and is re-stated below.

$$
r_{1 \rho}=\frac{q}{55.6}\left(\frac{R_{2 M} \times 1 / \tau_{M}}{R_{1 M}+1 / \tau_{M}}\right)
$$

It can be seen that by spin locking a component of laboratory frame transverse relaxation $\left(R_{2 \mathrm{M}}\right)$ is introduced and dominates over the laboratory frame longitudinal relaxation component $\left(R_{1 \mathrm{M}}\right) . \quad R_{2 \mathrm{M}}$ and $R_{1 \mathrm{M}}$, the transverse and longitudinal relaxation rate constants respectively, are defined in the theory of Solomon-Bloembergen-Morgan. $R_{2 \mathrm{M}}$ is shown below (Equation 4.1) considering only dipolar contribution; the scalar contribution is considered negligible as it is so small. ${ }^{87}$ Similarly, $R_{1 \mathrm{M}}$, previously described in Chapter 1 (Equation 1.20) is also shown for ease of reference. Terms in both $R_{2 \mathrm{M}}$ and $R_{1 \mathrm{M}}$ are the same as previously described.

$$
\begin{aligned}
& R_{2 M}=\frac{1}{15} \frac{\gamma_{I}^{2} g^{2} \mu_{B}^{2} S(S+1)}{r_{G d H}^{6}}\left[\frac{3 \tau_{c 1}}{1+\omega_{I}^{2} \tau_{c 1}^{2}}+\frac{13 \tau_{c 2}}{1+\omega_{S}^{2} \tau_{c 2}^{2}}\right]+4 \tau_{C 1} \\
& R_{1 M}=\frac{2}{15} \frac{\gamma_{I}^{2} g^{2} \mu_{B}^{2} S(S+1)}{r_{G d H}^{6}}\left[\frac{3 \tau_{c 1}}{1+\omega_{I}^{2} \tau_{c 1}^{2}}+\frac{7 \tau_{c 2}}{1+\omega_{S}^{2} \tau_{c 2}^{2}}\right]
\end{aligned}
$$

$R_{2 \mathrm{M}}$ has a larger dependence on the longitudinal correlation time constant $\left(\tau_{\mathrm{C} 1}\right)$ than $R_{1 \mathrm{M}}$, suggesting $\mathrm{r}_{1 \rho}$ should be more dependent on $\tau_{\mathrm{R}}$ and $\tau_{\mathrm{M}}$, according to Equation 1.19.

Equations 1.19, 1.20, 3.5 and 4.1 were used to simulate the rotating frame NMRD profiles of Magnevist and Dotarem, the "original" and "gold standard" agents, using well 
established physiochemical parameters at $25{ }^{\circ} \mathrm{C}$ obtained from decades of studying their laboratory frame NMRD. ${ }^{50}$ The observed data generated here was plotted against the theoretical simulation and is shown in Figure 4.3. It can be seen that the observed data (open symbols) agrees well with the simulations (lines), and that from approximately 20 $\mathrm{MHz}(0.47 \mathrm{~T})$ to $600 \mathrm{MHz}(14.1 \mathrm{~T})$ the simulation are almost entirely independent of $\mathrm{B}_{0}$, a consequence of the dominance of $R_{2 \mathrm{M}}$ in the $\mathrm{r}_{1 \rho}$ equation (Equation 3.5). In comparison to simulations for $r_{1}$ (Figure 1.15) over the same range of $\mathrm{B}_{0}$ fields, the $\mathrm{r}_{1} \rho$ simulations decrease much less rapidly, confirming our observations.

Magnetic Field (T)

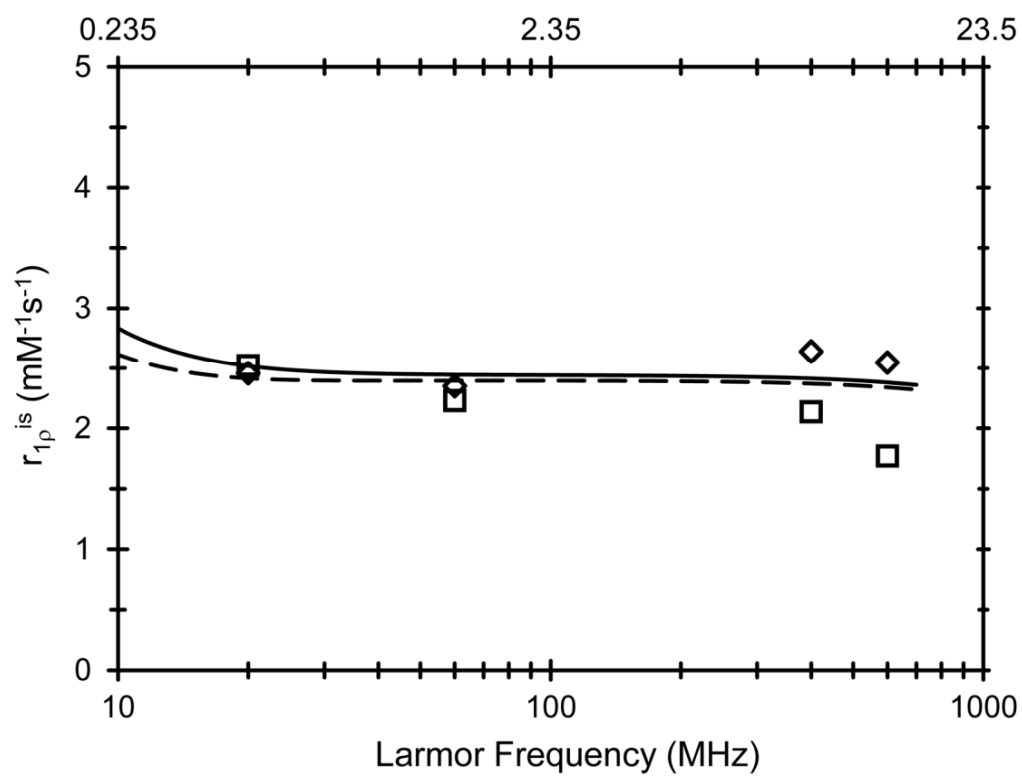

Figure 4.3: Rotating frame NMRD profiles of Magnevist and Dotarem in PBS at $25^{\circ} \mathrm{C}$. The lines through the data are simulations, using Eqns 1.20, 1.21, 3.5 and 4.1, of the rotating frame NMRD profile based on literature values of relevant parameters derived from laboratory frame NMRD analysis $; 00$ Dotarem: $\mathrm{q}=1 ; \mathrm{rgdH}=3.1 \AA ; \tau_{\mathrm{M}}=250 \mathrm{~ns}, \tau_{\mathrm{R}}=68.4 \mathrm{ps}, \Delta^{2}=1 \times 10^{19} \mathrm{~s}^{-2} ; \tau_{\mathrm{V}}=10 \mathrm{ps}$; Magnevist: $\mathrm{q}=1$, $\mathrm{rGdH}=3.1 \AA ; \tau_{\mathrm{M}}=303 \mathrm{~ns}, \tau_{\mathrm{R}}=67.9 \mathrm{ps}, \Delta^{2}=4.4 \times 10^{19} \mathrm{~s}^{-2} ; \tau_{\mathrm{V}}=24.9 \mathrm{ps}$; the outer sphere contribution was measured by analysis of GdTTHA ${ }^{3-}$. 


\subsection{A comparison of $r_{1}$ and $r_{1 \rho}$ for bulky $q=1$ clinical $G^{3+}{ }^{+}$based CAs}

Comparable experiments and data processing as described above for macrocyclic and linear agents was performed for bulky agents to determine their relaxivities and NMRD profiles. Due to the incorporation of bulky substituents to affect their biodistribution, Ablavar and MulitHance have slightly longer $\tau_{\mathrm{R}}$ values and elevated relaxivities when compared to the more rapidly tumbling linear and macrocyclic counterparts (Figure 4.4a and $\mathrm{b}$ at 25 and $37^{\circ} \mathrm{C}$, respectively). For this reason the bulky agents fare better than the linear and macrocyclic agents, particularly at high field, with an increase in relaxivity by a factor of 1.22 at $0.47 \mathrm{~T}$ on average, rising to 1.46 at $14.1 \mathrm{~T}$ on average (Table $4.5-4.6$ ).

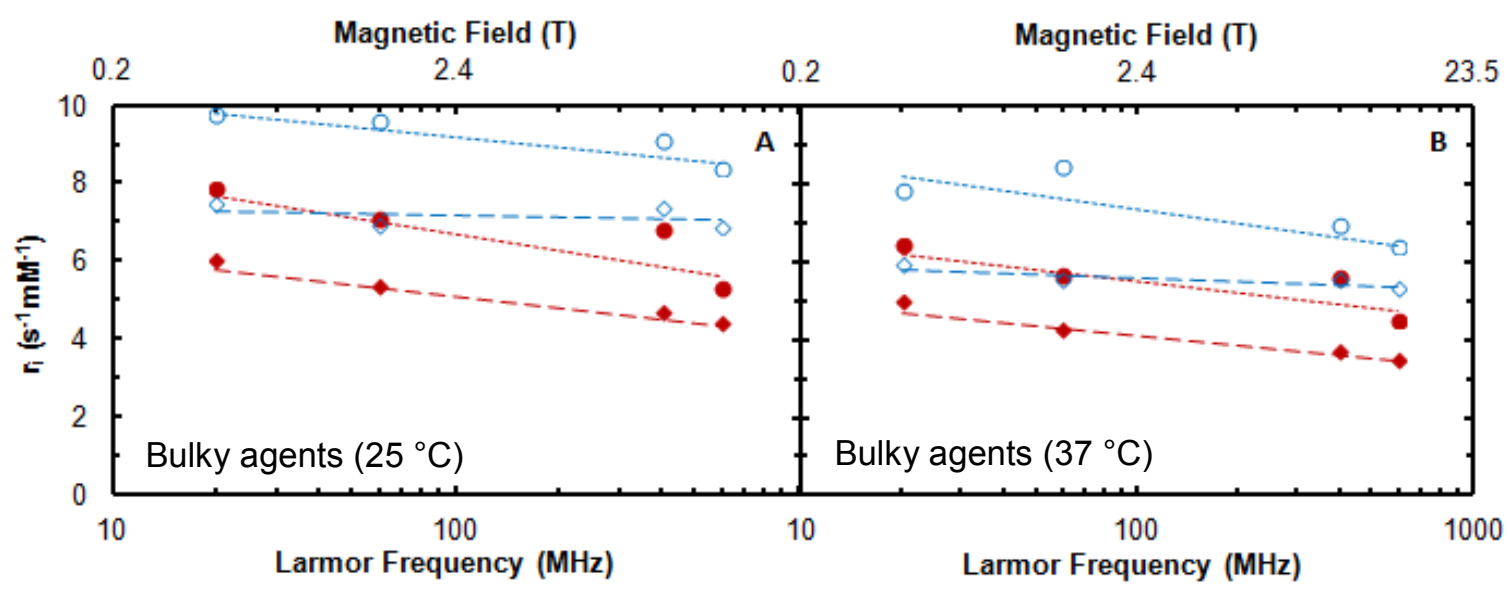

Figure 4.4: NMRD profiles of the bulky clinical contrast agents studied herein at $25^{\circ} \mathrm{C}(\mathrm{A})$ and $37^{\circ} \mathrm{C}(\mathrm{B})$ in PBS: laboratory frame data $\left(r_{1}\right)$ are presented in red (closed symbols); and rotating frame data $\left(r_{1 p}\right)$ are presented in blue (open symbols); $\omega_{1}=35 \mathrm{kHz}(0.47$ and $1.41 \mathrm{~T})$ and 6924.2 and $17237.0 \mathrm{~Hz}$ (9.4 and 14.1 T respectively). MultiHance (diamonds); Ablavar (circles), the green cross is the $r_{1}$ value calculated for Ablavar from reference. ${ }^{82}$ Lines through the data are to guide the eye only. Data shown are the mean of six measurements; error bars representing the $\mathrm{SD}$ of the data were omitted for clarity, all were smaller than the markers. 
As for linear and macrocyclic agents the $r_{1 \rho}$ values of bulky agents are higher than $r_{1}$ values for all fields investigated and both decrease as a function of $B_{0}$. However, their $r_{1 \rho}$ NMRD profiles decrease significantly more slowly than $r_{1}$ than was observed for the linear and macrocyclic agents, especially at lower temperatures.

The observed dependence of $r_{1 \rho}$ on the magnitude of the $\tau_{\mathrm{R}}$ value can be rationalized by referring to the theory described above and equations $1.20,3.5$ and 4.1 . These show that $r_{1 \rho}$ is more dependent than $r_{1}$ on the characteristic correlation time constant $\left(\tau_{\mathrm{C} 1}\right)$ of the contrast agent. Of the parameters in Equation $1.21 \tau_{\mathrm{R}}$ is limiting for $\mathrm{Gd}^{3+}$-based CAs such as those studied herein in which the fast water exchange regime criteria are met.

Comparable observations were made in the only other report of the use of spin locking with a $\mathrm{Gd}^{3+}$-based $\mathrm{CA}$ at high fields. ${ }^{82}$ In that report, Buckley and co-workers investigated spin locking at $4.7 \mathrm{~T}$ with Ablavar, known to bind reversibly to serum albumin, and observed significantly higher $R_{1 \rho}$ values when bound than when unbound. Although Buckley and co-workers do not quantify relaxivities in their study it is evident that $r_{1 \rho}$ values were greater than $r_{1}$ values. However, from the data presented this increases both the laboratory and rotating frame relaxivities of the agent.

By applying an analytical model used in a related study, ${ }^{88}$ we were able to estimate the bound and free rotating frame relaxivities for Ablavar with BSA, circumventing limitations of the method in which the data presented by Buckley and co-workers was generated. 
Table 4.5: Raw $r_{1}$ and $r_{1 \rho}$ data at 25 and $37{ }^{\circ} \mathrm{C}$ used to generate the NMRD above for bulky clinical agents studied herein at low field and their calculated ratios. Mean represents the average difference for the specified category of agents.

\begin{tabular}{|c|c|c|c|c|c|c|c|c|}
\hline & & & \multicolumn{2}{|c|}{$0.47 \mathrm{~T}$} & \multirow{2}{*}{$r_{1 \rho} / r_{1}$} & \multicolumn{2}{|c|}{$1.41 \mathrm{~T}$} & \multirow{2}{*}{$r_{1 \rho} / r_{1}$} \\
\hline & & & $r_{1}\left(\mathrm{mM}^{-1} \mathrm{~s}^{-1}\right)$ & $r_{1 \rho}\left(m M^{-1} s^{-1}\right)$ & & $r_{1}\left(\mathrm{mM}^{-1} \mathrm{~s}^{-1}\right)$ & $r_{1 p}\left(m M^{-1} s^{-1}\right)$ & \\
\hline \multirow{2}{*}{$25^{\circ} \mathrm{C}$} & \multirow{2}{*}{ Bulky } & MultiHance & 6.04 & 7.46 & 1.24 & 5.42 & 6.95 & 1.28 \\
\hline & & Ablavar & 7.86 & 9.75 & 1.24 & 7.11 & 9.63 & 1.35 \\
\hline & & Mean & & & 1.23 & & & 1.29 \\
\hline \multirow{2}{*}{$37^{\circ} \mathrm{C}$} & \multirow{2}{*}{ Bulky } & MultiHance & 5.01 & 5.95 & 1.19 & 4.32 & 5.57 & 1.29 \\
\hline & & Ablavar $^{a}$ & 6.44 & 7.89 & 1.23 & 5.70 & 8.47 & 1.49 \\
\hline & & Mean & & & 1.21 & & & 1.34 \\
\hline
\end{tabular}

${ }^{a}$ Free Ablavar 
Table 4.6: Raw $r_{1}$ and $r_{1 \rho}$ data at 25 and $37^{\circ} \mathrm{C}$ used to generate the NMRD above for bulky clinical agents studied herein at high field and their calculated ratios. Mean represents the average difference for the specified category of agents.

\begin{tabular}{|c|c|c|c|c|c|c|c|c|}
\hline & & & \multicolumn{2}{|c|}{$9.4 \mathrm{~T}$} & \multirow{2}{*}{$r_{1 \rho} / r_{1}$} & \multicolumn{2}{|c|}{$14.1 \mathrm{~T}$} & \multirow{2}{*}{$r_{1 \rho} / r_{1}$} \\
\hline & & & $r_{1}\left(\mathrm{mM}^{-1} \mathrm{~s}^{-1}\right)$ & $r_{1 p}\left(\mathrm{mM}^{-1} \mathrm{~s}^{-1}\right)$ & & $r_{1}\left(\mathrm{mM}^{-1} \mathrm{~s}^{-1}\right)$ & $r_{1 p}\left(\mathrm{mM}^{-1} \mathrm{~s}^{-1}\right)$ & \\
\hline \multirow{2}{*}{$25^{\circ} \mathrm{C}$} & \multirow{2}{*}{ Bulky } & MultiHance & 4.72 & 7.36 & 1.56 & 4.45 & 6.89 & 1.55 \\
\hline & & Ablavar & 6.84 & 9.1 & 1.33 & 5.30 & 8.39 & 1.58 \\
\hline & & Mean & & & 1.43 & & & 1.49 \\
\hline \multirow{2}{*}{$37^{\circ} \mathrm{C}$} & \multirow{2}{*}{ Bulky } & MultiHance & 3.76 & 5.59 & 1.49 & 3.52 & 5.34 & 1.52 \\
\hline & & Ablavar $^{\mathrm{a}}$ & 5.64 & 6.97 & 1.24 & 4.53 & 6.44 & 1.42 \\
\hline & & Mean & & & 1.37 & & & 1.42 \\
\hline
\end{tabular}

${ }^{\mathrm{a}}$ Free Ablavar 
The model we used assumes a 1:1 binding interaction and uses a binding constant derived empirically $\left(\mathrm{K}_{\mathrm{a}}=12 \mathrm{M}^{-1}\right) .{ }^{89}$ Fitting the Buckley data to this model gave values of $r_{1 p}$ bound $=84.8 \mathrm{mM}^{-1} \mathrm{~s}^{-1}$ and $r_{1 \mathrm{p}}$ free $=20.8 \mathrm{mM}^{-1} \mathrm{~s}^{-1}$. The bound $r_{1 \rho}$ value compares favorably with the bound $r_{1}$ value reported in literature $\left(16.2 \mathrm{mM}^{-1} \mathrm{~s}^{-1}\right)$ for the same agent with human serum albumin (HSA) but at slightly lower field and temperature $\left(3 \mathrm{~T}\right.$ and $\left.25^{\circ} \mathrm{C}\right)$.

The substantial increase of both laboratory and rotating frame relaxivity on binding to HSA or BSA is a direct consequence of the increase in the effective molecular weight of the agent and a lengthening of $\tau_{R}$. It is worthwhile commenting on the magnitude of the free $r_{1 \rho}$ calculated in the presence of BSA. Since the original experiments were performed in the presence of $4.5 \% w / v$ of BSA, an increase in viscosity is likely which further lengthens $\tau_{R}$ resulting in elevated relaxivities, particularly in the rotating frame which we have shown to be more sensitive to $\tau_{R}$.

\subsection{Summary of relaxivity changes}

Figure 4.5 shows that, in the laboratory frame (red), an approximate 20 and $30 \%$ decrease in relaxivity is observed for low molecular weight (solid) and bulky (diagonal lines) clinical agents respectively when the external field strength is increased from $0.47 \mathrm{~T}$ $(\sim 0.5 \mathrm{~T})$ to $14.1 \mathrm{~T}$. This is a consequence of these agents having comparable laboratory frame relaxivities at $14.1 \mathrm{~T}$, whereas at $0.5 \mathrm{~T}$ bulky agents have higher laboratory frame relaxivities. Figure 4.5 allows for a better appreciation of the increases in relaxivity by spin locking clinical agents demonstrated in Figures 4.1 and 4.4. At $0.5 \mathrm{~T}$ (purple) the 
increase is modest for both low molecular weight and bulky agents with an increase of approximately $20 \%$ for each. At $14.1 \mathrm{~T}$ (green) more substantial increases are observed, with low molecular weight agents increasing by approximately $35 \%$ and bulky agents increasing by approximately $65 \%$. As discussed previously, the increased gains at higher fields are a result of a less rapid decrease in $\mathrm{r}_{1 \rho}$ as a function of $\mathrm{B}_{0}$ than that observed for $r_{1}$ (Figure 4.1 and 4.4).

By comparing the change in relaxivity between low molecular weight agents (short $\tau_{R}$ ) and bulky agents (long $\tau_{\mathrm{R}}$ ) Figure 4.10 clearly demonstrates our finding that $r_{1 \rho}$ is more sensitivity than $r_{1}$ to the rotational correlation time $\left(\tau_{\mathrm{R}}\right)$, particularly at high field. Figure 4.5 also demonstrates that by spin locking at the high field strengths of the future, the loss of relaxivity observed by remaining in the laboratory frame (red) can not only be recovered, but increased (blue). Additionally, this increase occurs to a greater extent for bulky agents $(\sim 10 \%$ increase in relaxivity) than for low molecular weight agents $(\sim 5 \%)$.

\subsection{Conclusions}

We were able to demonstrate in the presence of an optimized spin lock pulse that relaxivity lost on moving to higher $B_{0}$ field strengths was able to be "recovered" across all $B_{0}$ field strengths studied and for all $\mathrm{Gd}^{3+}$-based CAs in current clinical use at both 25 and $37{ }^{\circ} \mathrm{C}$. For now, it remains to be seen if these findings will afford increased detectability and reduced limits of detection of these agents. 


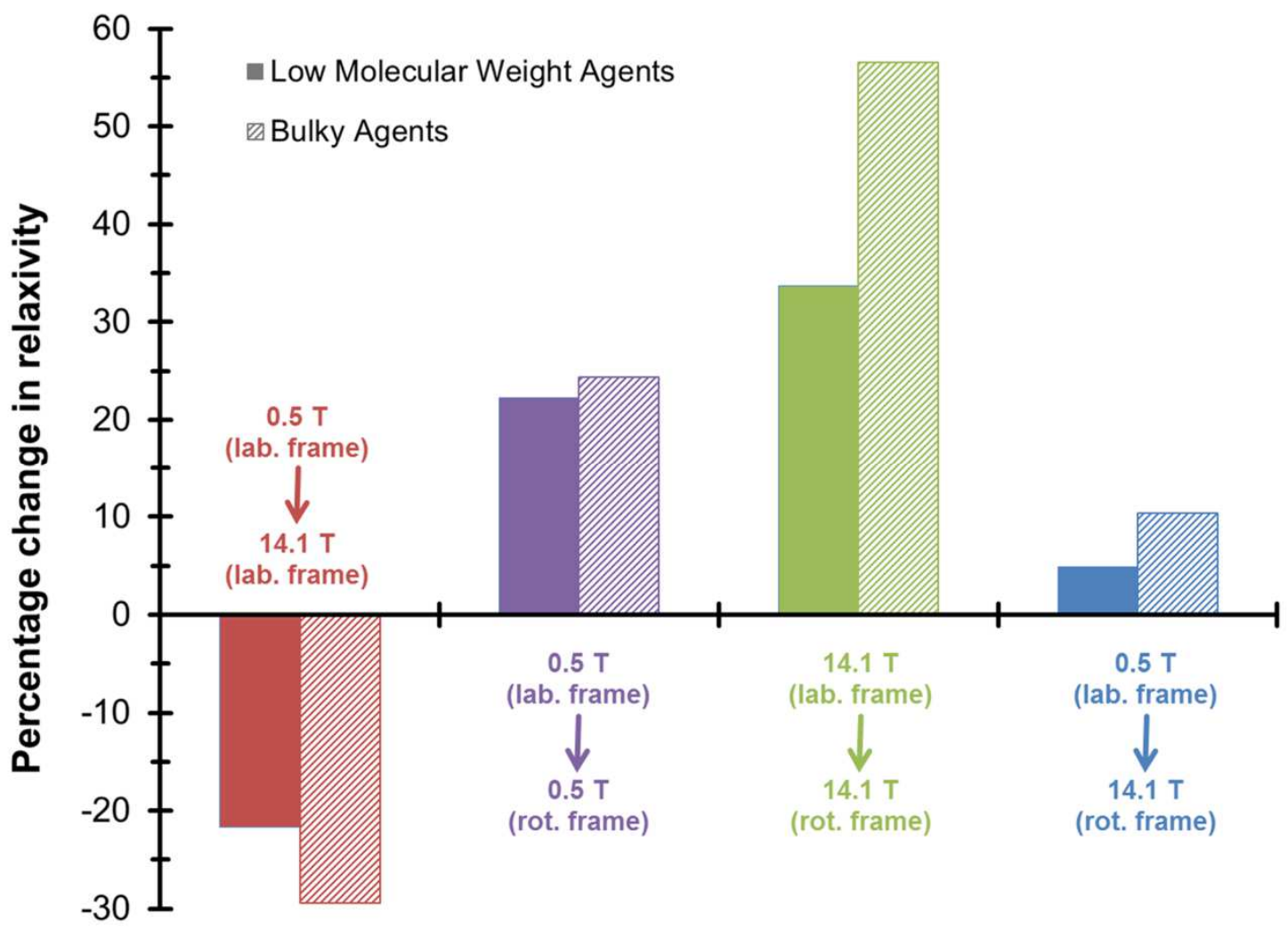

Figure 4.5: Percent change in relaxivity at $25^{\circ} \mathrm{C}$ by spin locking (rot. frame) at low field (Purple), high field (green) and over the entire range of field strengths studied (blue) for low molecular weight agents (solid) and bulky agents (diagonal lines). For reference the percent change in relaxivity without spin locking (lab. frame) over the entire range of field strengths studied (red) is also shown for the same agents.

However, it is known that the effect the spin locking pulse will have on inherent rotating frame relaxation rate constants $\left(R_{1}\right)$ of tissue at these fields will play a role in achieving this particular goal. It can be theorized that since spin locking has a large $R_{2}$ contribution, inherent tissue $R_{1 \rho}$ values may be comparable. At field strengths up to $2.35 \mathrm{~T}(100 \mathrm{MHz})$ inherent tissue $R_{2}$ values have been reported to be fairly independent of $B_{0}$ in both normal and pathological tissue. ${ }^{90-91}$ However, at higher $B_{0}$ field strengths, such as those 
investigated herein, inherent tissue $R_{2}$ values have been shown to increase. ${ }^{92}$ This suggests that agent detectability could be increased at such fields as a result of the less rapid decrease in relaxivities observed at these high fields by spin locking.

Our finding that the rotating frame relaxivity $\left(r_{1 \rho}\right)$ is especially sensitive to the rotational correlation time $\left(\tau_{R}\right)$ is of particular interest. This effect is more pronounced at high $B_{0}$ fields, where substantial loss of relaxivity is observed and to which future clinical imaging is heading. Spin locking is therefore likely to be of interest to researchers in the field of molecular imaging. In such applications, agents are designed to target large molecular weight biomarkers thereby increasing their effective molecular weight substantially. By employing a spin locking pulse with such agents it may be possible, at high field, to "recover" relaxivity in remarkably larger amounts than any gains possible from similar agents in the laboratory frame. This hypothesis if proven to be true could potentially decrease the limits of detection of such agents to levels required for successful molecular imaging where biomarkers typically exist at very low concentrations. 


\section{CHAPTER 5. EFFECT OF ROTATIONAL CORRELATION TIME $\left(\tau_{R}\right)$ ON ROTATING FRAME LONGITUDINAL RELAXIVITY ( $\left.\boldsymbol{r}_{1 \rho}\right)$}

\subsection{Establishing the potential for increasing relaxivity by spin locking slow tumbling nano-encapsulated $\mathrm{Gd}^{3+}$ chelates.}

The NMRD profiles for $\mathrm{q}=1$ clinical $\mathrm{Gd}^{3+}$-based CAs (Figures 4.1 and 4.7 ) and summary (Figure 4.10), demonstrate that lost relaxivity on moving to higher $B_{0}$ fields could be "recovered" by using a spin locking pulse and measuring the rotating frame relaxivity $\left(r_{1 \rho}\right)$. Comparison of $r_{1 \rho}$ and $r_{1}$ NMRD profiles for all three classes of clinical agents revealed that $r_{1 \rho}$ is more sensitive to the rotational correlation time $\left(\tau_{R}\right)$ than $r_{1}$. Evidence for this was shown by measuring greater gains in relaxivity on applying a spin locking pulse with low molecular weight bulky agents that tumble more slowly than were measured for low molecular weight non-bulky agents. This was particularly apparent at high $B_{0}$ fields where substantial loss of laboratory frame relaxivity is typically observed and towards which clinical imaging is heading. However, due to only a slight difference in $\tau_{\mathrm{R}}$ between the low molecular weight non-bulky and bulky agents studied, the relaxivity gains from slowing tumbling were fairly modest.

Using the SBM equations it has been possible to model the inner sphere contribution to $r_{1}$ and $r_{1 \rho}$ for a theoretically "optimized" gadoterate chelate for which $\tau_{\mathrm{R}}$ has been increased from 68.4 ps to $68.4 \mathrm{~ns}$ and $\tau_{\mathrm{M}}$ has been reduced from $250 \mathrm{~ns}$ to $25 \mathrm{~ns}$ (Figure 5.1). 


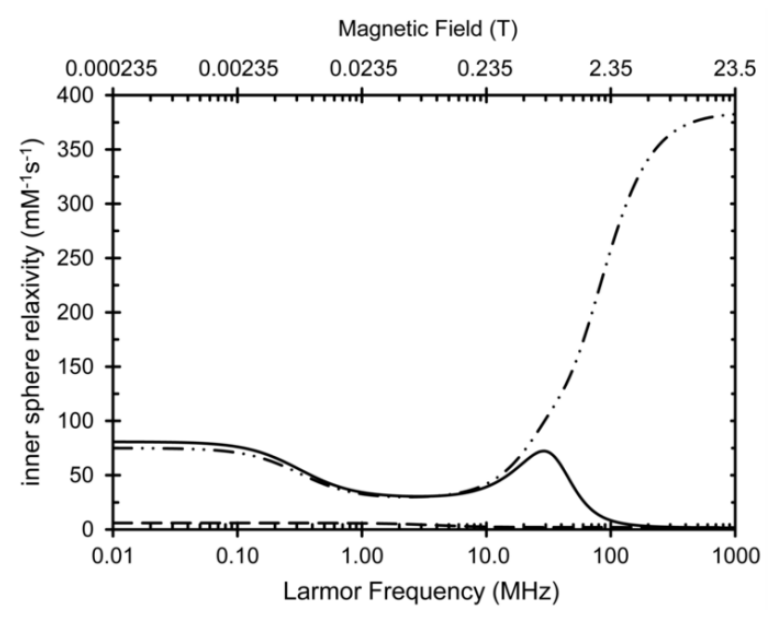

Figure 5.1: Nuclear magnetic relaxation dispersion (NMRD) profile, generated by us, plotting the inner sphere contribution to the laboratory frame (solid line) and rotating frame (dashed-dot-dot line) longitudinal relaxivity for a theoretically "optimized" gadoterate chelate for which $\tau_{R}$ has been increased from $68.4 \mathrm{ps}$ to $68.4 \mathrm{~ns}$ and $\tau_{\mathrm{M}}$ reduced from $250 \mathrm{~ns}$ to $25 \mathrm{~ns}$. Also shown is the NMRD profile of gadoterate (dashed line ${ }^{61}$ ) plotting the inner sphere contribution to the laboratory frame longitudinal relaxivity as a function of $B_{0}$ field.

At around $0.71 \mathrm{~T}(30 \mathrm{MHz}), r_{1}$ (solid line) for an optimized gadoterate chelate shows a steady and continual decrease as $B_{0}$ increases. This occurs to such an extent that $r_{1}$ converges at around 4.7 $\mathrm{T}(200 \mathrm{MHz})$, the upper limit of current clinical imaging fields, with the $r_{1}$ value of a nonoptimal chelate (dashed line) and all contrast agents are found to behave identically. However, $r_{1 \rho}$ (dashed-dot-dot line) increase rapidly from $0.71 \mathrm{~T}$ (30 $\mathrm{MHz})$ up to around 4.7 $\mathrm{T}(200 \mathrm{MHz})$ after which it increases more slowly up to $23.5 \mathrm{~T}$ $(1000 \mathrm{MHz})$. This demonstrates the potential for $r_{1} \rho$ to become exceedingly high at very high $B_{0}$ field strengths if molecular tumbling can be substantially slowed and water exchange optimized. Spin locking may provide a method for significantly increasing the detectability of such $\mathrm{Gd}^{3+}$-based CAs. This would be useful in applications such as molecular imaging where biomarkers exist at very low concentrations. 


\subsection{Nanoassembled capsules as a model system for investigating the effect of $\tau_{R}$ on $r_{1 \rho}$}

Novel high molecular weight nanoassembled capsules (NACs) containing a $\mathrm{Gd}^{3+}$-based CA represent a convenient model for investigating the effect of making $\tau_{R}$ longer. Samples of $\mathrm{Gd}^{3+}$ containing NACs were kindly supplied by Annah Farashishiko. The particular advantage of these capsules are that they afford well-defined, slowly rotating systems that are uncomplicated by the presence of binding equilibria. We therefore investigated NACs of different size comparing their $r_{1}$ and $r_{1 \rho}$ values at high $B_{0}$ field strengths, 9.4 and $14.1 \mathrm{~T}$ (400 and $600 \mathrm{MHz}$ respectively), at $25^{\circ} \mathrm{C}$. Low field $r_{1}$ values were also determined; however, due to instrument and sample limitations (short spin lock pulse durations and low $\mathrm{Gd}^{3+}$ concentrations) it was not possible to determine low field relaxivity in the rotating frame.

\subsection{Mechanism of action of nanoassembled capsules (NACs) incorporating GdDOTP ${ }^{5-}$}

GdDOTP $^{5-} \quad\left(\right.$ DOTP $=1, \quad 4, \quad 7, \quad$ 10-tetra-azacyclododecane- $\quad N, N^{\prime}, N^{\prime \prime}, N^{\prime \prime \prime}-$ tetrakis(methylenephosphonic acid)) is a highly charged $q=0$ chelate with a relaxivity of $4.7 \mathrm{mM}^{-1} \mathrm{~s}^{-1}\left(23 \mathrm{MHz}, 25^{\circ} \mathrm{C}\right),{ }^{93}$ remarkably high considering the contributions are solely from second and outer sphere water. This has largely been attributed to the ability of the phosphonate groups to tightly bind water ${ }^{94}$ and form an ordered second sphere ${ }^{95}$ with extensive hydrogen bonding that slows water exchange $\left(1 / \tau_{\mathrm{M}}\right)$ (Figure 5.2). This negatively charged anionic chelate forms aggregates with high molecular weight cationic polymers which when encapsulated with $\mathrm{SiO}_{2}$ form NACs (Figure 5.3). 


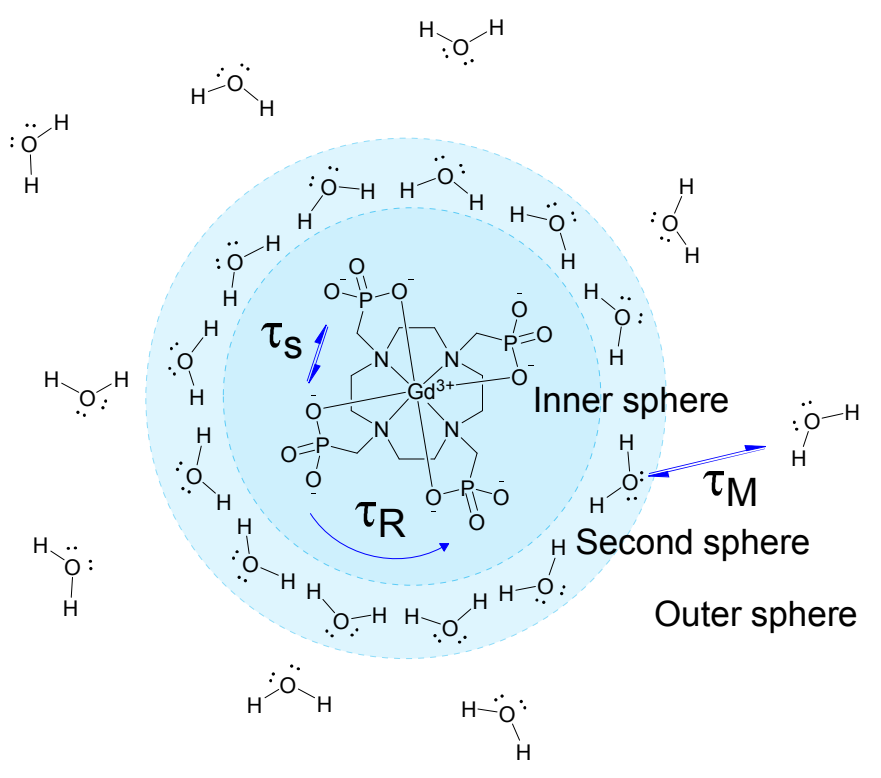

Figure 5.2: [GdDOTP] $]^{5-}$ a $q=0$ complex.

By encapsulating GdDOTP ${ }^{5-}$ with a $\mathrm{SiO}_{2}$ nano-particle the outer sphere contribution to relaxivity is essentially eliminated rendering the capsules as purely second sphere agents. Additionally, the second sphere water exchange rate is further reduced by aggregate formation. The encapsulating shell does not inhibit water exchange to such an extent that it limits relaxivity, i.e. water remains in the fast exchange regime. ${ }^{93}$ Second sphere contributions can be described by SBM theory (used to quantify inner-sphere contributions) and the typical governing parameters, $\tau_{M}$ and $\tau_{R}$, albeit with an elongated $r_{\mathrm{Gd}-\mathrm{H}}$ distance. It has been shown that at magnetic field strengths of $0.47-1.41 \mathrm{~T}$ the limiting effect of $\tau_{R}$ is lifted by the lengthening of $\tau_{R}$ on forming aggregates and NACs and that under these conditions the laboratory frame relaxivities are elevated. ${ }^{93}$ 


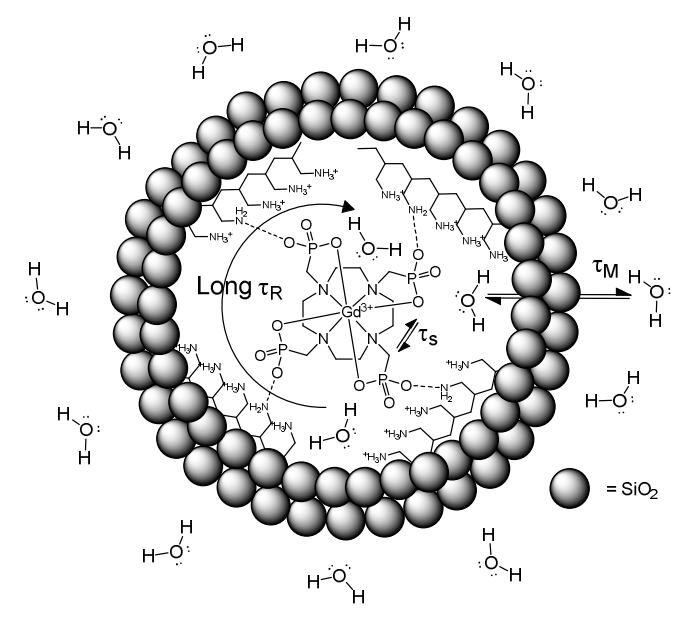

Figure 5.3: Cartoon representation of a $\mathrm{Gd}^{3+}$ based NAC.

\subsection{Effect of NAC design parameters on relaxivity}

Relaxivity is dependent on the size of the NACs. Smaller NACs give rise to the greatest gains in relaxivity owing to the increased facility for proton transport between chelate and bulk water - the result of shorter distances required to travel by the water in order to cross the capsule shell and transmit the paramagnetic relaxation enhancement to the bulk. The size of NACs have been shown to be dependent on three critical design parameters: the charge ratio, the cationic polymer and the solvent system used in their synthesis. ${ }^{93}$ We chose to study NACs prepared with polyallylamine hydrochloride (PAH) in 3:2 $\mathrm{MeCN} / \mathrm{H}_{2} \mathrm{O}$ at a charge ratio (R) of $0.3,0.5$ and 1 with the penta-anionc chelate $\left(\right.$ GdDOTP $\left.^{5-}\right)$. These design parameters were chosen in an effort to maximize their relaxivities by limiting their size. Representative SEM images of NACs at each charge ratio studied are shown in Figure 5.4 and their respective relaxivities in Table 5.1. 


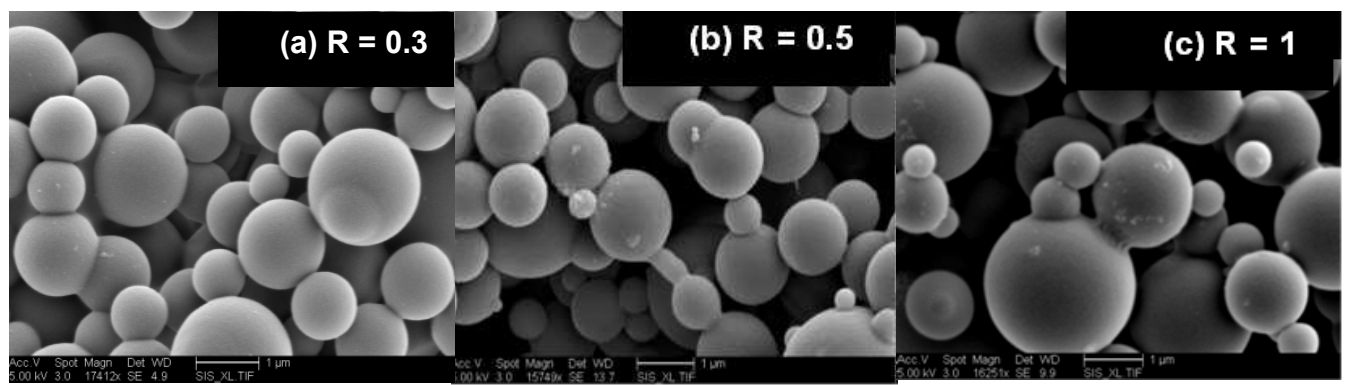

Figure 5.4: SEM of PAH-GdDOTP5--based NACs prepared in $\mathrm{MeCN} / \mathrm{H}_{2} \mathrm{O}, 3: 2$ at three different charge ratios $(R)$.

Table 5.1: Laboratory frame longitudinal relaxivity $\left(r_{1}\right)$ data for the PAH-GdDOTP5--based NACs shown in Figure $5.4\left(25^{\circ} \mathrm{C}\right.$ at $\left.20 \mathrm{MHz}\right)$.

\begin{tabular}{|l|c|c|c|}
\hline & $\mathbf{R}=\mathbf{0 . 3}$ & $\mathbf{R}=\mathbf{0 . 5}$ & $\mathbf{R}=\mathbf{1}$ \\
\hline $\boldsymbol{r}_{\mathbf{1}}\left(\mathrm{mM}^{-1} \mathbf{s}^{-1}\right)$ & 44.39 & 22.36 & 7.88 \\
\hline
\end{tabular}

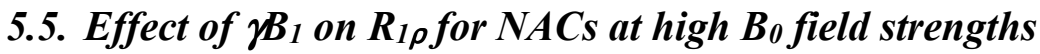

According to our model (Figure 5.1 dashed-dot-dot line) the rotating frame longitudinal relaxivity for an optimized agent should be very high at high fields, therefore, we investigated the effect of $\gamma B_{1}$ on $R_{1}$ for NACs at $600 \mathrm{MHz}(14.1 \mathrm{~T})$ and $25^{\circ} \mathrm{C}$. For this a sample of NACs with charge ratio (R) of 0.5 encapsulating $\mathrm{GdDOTP}^{5-}$ at a $\mathrm{Gd}^{3+}$ concentration of $0.89 \mathrm{mM}$ in water were used. The strength of the spin locking field $\left(\gamma B_{1}\right)$ was modulated by attenuating the power. For comparison a sample of the free chelate at a $\mathrm{Gd}^{3+}$ concentration of $5 \mathrm{mM}$ was also investigated. The Rabi frequencies that 
characterize $\gamma B_{1}$ over the attenuation range sampled corresponded to $\sim 200-20000 \mathrm{~Hz}$ (NACs) and $\sim 400-20000 \mathrm{~Hz}\left(\mathrm{GdDOTP}^{5-}\right)$ the results are shown in Figure 5.5.

As expected for the free chelate, similar facets to those observed for Dotarem (Figure 3.2), also a low molecular weight $\mathrm{Gd}^{3+}$-chelate, at high $B_{0}$ fields can be identified. Namely, the rotating frame longitudinal relaxation rate $\left(R_{1} \rho\right)$ of GdDOTP ${ }^{5-}$ showed very low errors associated with the data but the mean data point did start to show some scatter at the lower spin lock field strengths studied. Also, the strength of the spin lock pulse required to effectively lock the spins at high $B_{0}$ field was approximately the same, $\sim 500$ Hz. Again these values are much more realistic than those determined from our low field experiments. The reduced spin lock field strength required to effectively spin lock, compared to low field experiments, can again be attributed to the shim capabilities of the superconducting instrument and the low $\mathrm{Gd}^{3+}$ concentration employed that both contribute to narrow line-widths and low pulse strengths being required to spin lock.

For the GdDOTP ${ }^{5-}$ encapsulated NACs, the errors associated with the data are again very low, however the data is shown to be dependent on the strength of the spin lock pulse with an increasing trend in the rotating frame relaxation rate constant as the power is decreased up to $\sim 1000 \mathrm{~Hz}$ where an inflection in the data occurs and $R_{1 \rho}$ starts to decrease as powers continue to decrease to $\sim 200 \mathrm{~Hz}$. It is important to keep in mind that equation 3.1 derived from the theory of Jordan and co-workers ${ }^{70}$ does not apply in the case of an encapsulated $\mathrm{Gd}^{3+}$ chelate, the chelate no longer being in simple exchange with water. 


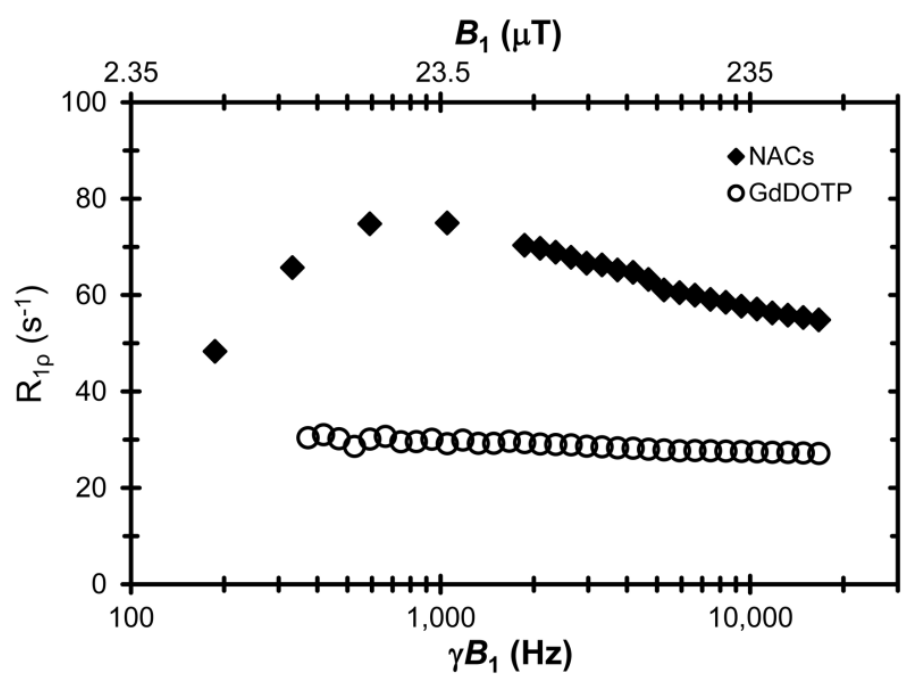

Figure 5.5: The dependence of the water proton rotating frame longitudinal relaxation rate constant $\left(R_{1 p}\right)$ on the spin lock field strength $\left(\gamma B_{1}\right)$ for a sample of $\mathrm{R}=0.5 \mathrm{PAH}-G \mathrm{dDOTP} \mathrm{P}^{5-}$ encapsulated NACs (filled circles) and the free chelate (open circles) in water $14.1 \mathrm{~T}(600 \mathrm{MHz})$ and $25^{\circ} \mathrm{C}$. Data shown are the mean of six measurements; error bars representing the SD of the data were ommited for clarity but were all less than the markers.

\subsection{A comparison of laboratory $\left(r_{1}\right)$ and rotating ( $\left.r_{1} \rho\right)$ frame NMRD for NACs}

Laboratory $\left(r_{1}\right)$ and rotating $\left(r_{1 \rho}\right)$ frame relaxivities for NACs prepared in this study at several charge ratios were determined over a range of $B_{0}$ field strengths and their NMRD profiles generated. For comparative purposes, free un-encapsulated GdDOTP ${ }^{5-}$ was also measured (Figure 5.6).

\subsubsection{Effect of $\tau_{R}$ on $r_{1}$ as a function of $B_{0}$}

As predicted by our model for a theoretically "optimized" agent (Figure 5.1, solid line) $r_{1}$ values for NACs (Figure 5.6, red open symbols) decrease on increasing the field strength 
from $0.47 \mathrm{~T}(20 \mathrm{MHz})$ to $1.41 \mathrm{~T}(60 \mathrm{MHz})$ after likely reaching a maximum around $0.7 \mathrm{~T}$ (30 MHz).

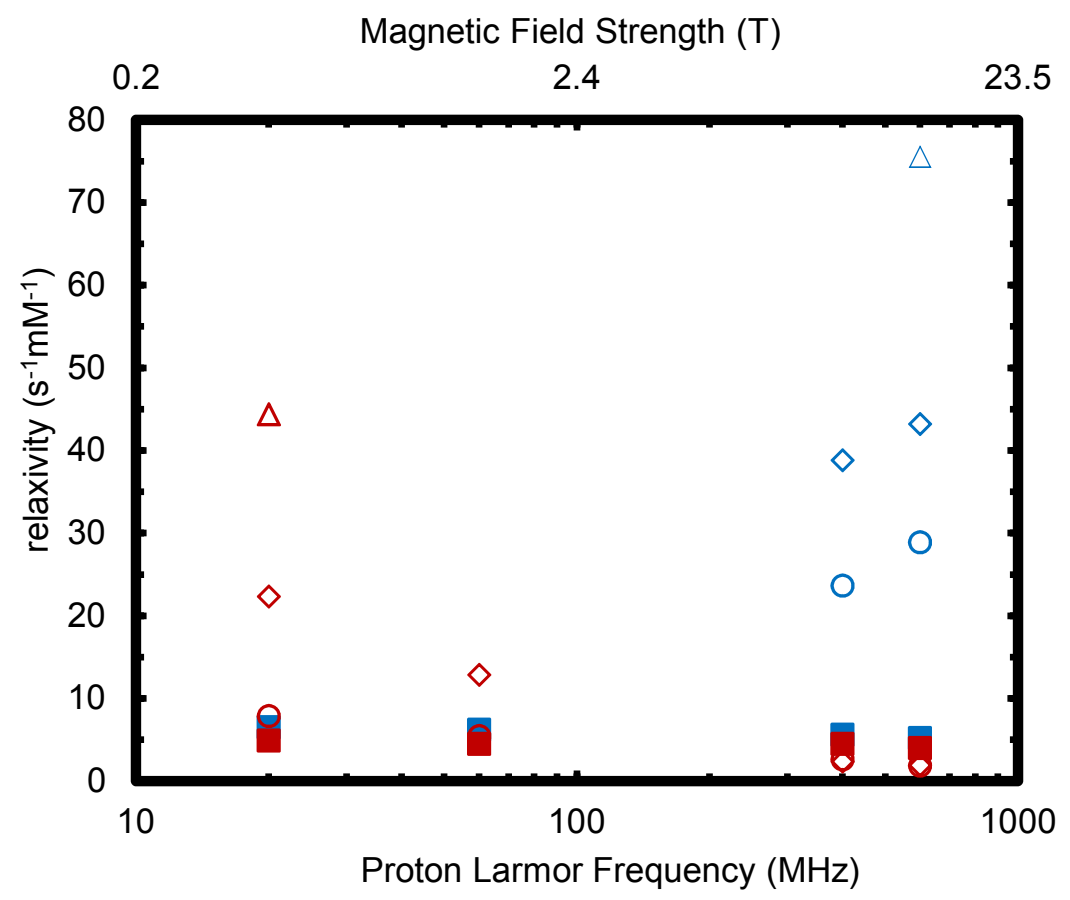

Figure 5.6: NMRD profiles of NACs at $25^{\circ} \mathrm{C}$ in water: laboratory $\left(r_{1}\right)$ and rotating $\left(r_{1 p}\right)$ frame data are presented in red and blue (open symbols) respectively. $\omega_{1}=35 \mathrm{kHz}(0.47$ and $1.41 \mathrm{~T})$ and 6924. 2 and $17237.0 \mathrm{~Hz}$ (9.4 and 14.1 T respectively). Charge ratio (R): 1 (circles), 0.5 (diamonds) and 0.3 (triangles). Laboratory $\left(r_{1}\right)$ and rotating $\left(r_{1 \rho}\right)$ frame data are also presented in red and blue (closed squares) respectively for un-encapsulated GdDOTP5-. Data shown are the mean of three measurements; error bars representing the SD of the data were omitted for clarity, all were smaller than the markers except $r_{1 \rho}$ for $\mathrm{R}=0.3$ NACs which are slightly larger than the marker. Note: $r_{1 \rho}$ for $R=0.3 \mathrm{NACs}$ is based on an estimated $\left[\mathrm{Gd}^{3+}\right]$, at the time of writing ICPAES data was not avaialble.

The $r_{1}$ values continue to decrease as the field strength is increased further to $9.4 \mathrm{~T}$ (400 $\mathrm{MHz})$ and $14.1 \mathrm{~T}(600 \mathrm{MHz})$, again, consistent with the model and presumably due to sub-optimal water exchange at these fields in the laboratory frame. 
The $r_{1}$ values for the free chelate (red closed squares) are consistent with the model for a "non-optimized" low molecular weight non-bulky chelate (Figure 5.1, dashed line) and previous observations for such chelates studied herein. Consistent with previous observations for similar systems by our group, ${ }^{93} r_{1}$ values for NACs (red open symbols) at low field, $0.47 \mathrm{~T}(20 \mathrm{MHz})$, are elevated over those of the free chelate (red closed squares) as is the case at $1.41 \mathrm{~T}(60 \mathrm{MHz})$, a consequence of the slowing of rotation. At high fields, it might have been expected that the $r_{1}$ values for NACs and free chelate would converge on approximately the same value. However, this is not the case as laboratory frame relaxivity values for NACs were found to be lower than the respective values for the free chelate. This may be the result of two factors. Firstly, by eliminating the outer sphere contribution by encapsulation, the theoretical relaxivity possible for NACs would be expected to be lowered by approximately half compared to the free chelate. ${ }^{22}$ Secondly, water exchange for the NACs is likely to be slower, due to the encapsulation that provides a boundary to exchange, and thus less optimum at these field strengths than for the free chelate.

Also consistent with our previous findings with similar systems ${ }^{93}, r_{1}$ increases as the charge ratio, proportional to size, decreases from 1 (red open circles) to 0.5 (red open diamonds) and further to 0.3 (red open triangles) at low fields. This is a result of more effective rotational restriction and increased water exchange rates $\left(1 / \tau_{\mathrm{M}}\right)$ in smaller NACs. This was not observable at the higher fields studied herein due to the non- 
optimum water exchange rates limiting the relaxivity at these fields leading to negligible difference in the $r_{1}$ values between the NACs with different charge ratios.

\subsubsection{Effect of $\tau_{R}$ on $r_{l \rho}$ as a function of $B_{0}$}

With the low field relaxometers the maximum spin lock pulse duration is $40 \mathrm{~ms}$. This rendered it impossible to determine $r_{1 \rho}$ values of NACs at 0.47 and $1.41 \mathrm{~T}(20$ and 60 MHz) with the low sample concentrations employed. The higher concentrations needed for these experiments were not possible due to material limitations.

From the high field portion of the NMRD profiles in Figure 5.6 (9.4 - 14.1 T, $400-600$ $\mathrm{MHz}$ ) it can be seen that $r_{1 \rho}$ values for NACs (blue open symbols) are substantially elevated over the respective $r_{1}$ values (red open symbols) as observed for the clinical agents discussed previously. However, and more importantly, the $r_{1 \rho}$ values increase on moving to higher $B_{0}$ fields rather than decrease as for $r_{1}$. This finding is in agreement with predictions made by our model for a theoretically "optimized" gadoterate chelate in the rotating frame (Figure 5.1, dashed-dot-dot). These results demonstrate the potential benefits of applying spin locking pulses when imaging using slowly tumbling $\mathrm{Gd}^{3+}$-based contrast media at high magnetic field strengths.

As observed for other low molecular weight non-bulky agents in this work the rotating frame relaxivity of the free chelate (blue closed squares) was elevated over the laboratory frame relaxivity (red closed squares) at all $B_{0}$ field strengths studied and decreased as $B_{0}$ increased. 
At high fields $r_{1 \rho}$ values for NACs (blue open symbols) are greater than that of the respective values for the fast tumbling free chelate (blue closed squares). This is again a consequence of the significantly longer $\tau_{\mathrm{R}}$ for NACs over that of the free chelate.

High field $r_{1 \rho}$ values for NACs (blue open symbols) also exhibit a substantial increase as the charge ratio (size) decreases from 1 (blue open circles) to 0.5 (blue open diamonds) and further to 0.3 (blue open triangles). Again, this is a consequence of smaller capsules providing more effective rotational restriction and increased water exchange rates.

\subsection{Summary of relaxivity changes}

Figure 5.7 shows that, in the laboratory frame (red), an approximate 75, 90 and 95\% decrease in relaxivity is observed for NACs prepared with a charge ratio of $\mathrm{R}=1$ (checker board), 0.5 (diagonal brick) and 0.3 (confetti) respectively when the external field strength is increased from $0.47 \mathrm{~T}(0.5 \mathrm{~T})$ to $14.1 \mathrm{~T}$. For comparison, the change in relaxivity in the laboratory frame for clinical agents, $\sim 25 \%$ (solid), and the GdDTOP ${ }^{5-}$ free chelate, $\sim 20 \%$ (diagonal lines), are also shown. The greater decrease in relaxivity for NACs compared to the clinical agents and free chelate is a consequence of NACs having different, and substantially elevated, laboratory frame relaxivities at $0.5 \mathrm{~T}$ over the clinical agent and free chelate (Figure 5.6) but comparable laboratory frame relaxivities at $14.1 \mathrm{~T}$. Figure 5.7 allows for a better appreciation of the increase in relaxivity by spin locking NACs at high field demonstrated in Figure 5.6. At 14.1 $\mathrm{T}$ (green) the increases are exceptionally large for all NACs, approximately 600,1000 and $1800 \%$ for $\mathrm{R}=1,0.5$ 
and 0.3 respectively, and much more significant than the respective increases observed for clinical agents (solid) and the free chelate (diagonal lines). The NAC values were calculated relative to the $r_{1}$ value for the free chelate since this is greater than the $\mathrm{r}_{1}$ value for NACs at these fields (Figure 5.6) a consequence of NACs having less optimum water exchange. This clearly demonstrates the sensitivity of $r_{1 \rho}$ to the rotational correlation time $\left(\tau_{R}\right)$ which is lengthened on decreasing the NAC size, proportional to charge ratio, due to more effective rotational restriction and increased water exchange rates. Due to instrument and sample limitations discussed previously, it was not possible to measure rotating frame relaxivities on our low field instruments, therefore a comparison to $r_{1}$ at $0.5 \mathrm{~T}$ could not be made. Figure 5.7 also demonstrates that by spin locking at the high field strengths of the future, lost relaxivity that is expected by remaining in the laboratory frame (red) for clinical agents, free chelate or NACs, can not only be recovered but increased (blue). Additionally, this increase occurs to a greater extent for NACs ( $\sim 260$, 90 and $60 \%$ for $\mathrm{R}=1,0.5$ and 0.3 respectively) than for clinical agents $(\sim 8 \%)$ and free chelate $(\sim 6 \%)$. The decrease in the change in relaxivity for NACs as a function of the charge ratio (blue) is a consequence of larger increases in $r_{1}$ at $0.5 \mathrm{~T}$ than $\mathrm{r}_{1 \rho}$ at $14.1 \mathrm{~T}$ (Figure 5.6).

\subsection{Conclusions}

Rotating frame relaxivities at high fields were found not only to be exceptionally large compared to those obtained in the laboratory frame $\left(43 \mathrm{mM}^{-1} \mathrm{~s}^{-1}\right.$ vs. $1.9 \mathrm{mM}^{-1} \mathrm{~s}^{-1}$ for NACs with a charge ratio of 0.5 at $14.1 \mathrm{~T}$ ), but also increased as $B_{0}$ increased. 


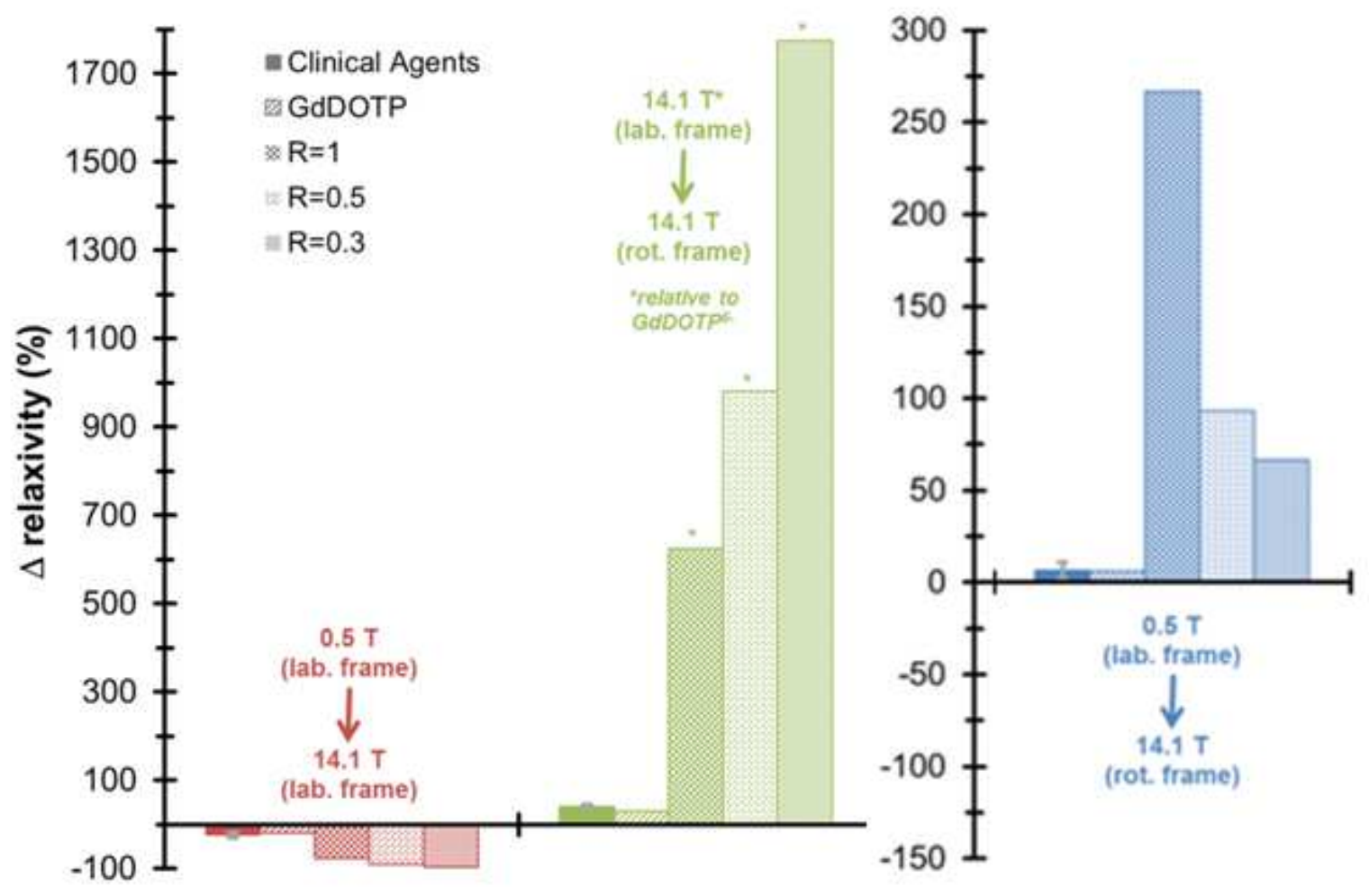

Figure 5.7: Percent change in relaxivity at $25^{\circ} \mathrm{C}$ by spin locking (rot. frame) at high field (green) and over the entire range of field strengths studied (blue) for clinical agents (solid), GdDOTP5free chelate (diagonal lines) and GdDOTP5- NACs $R=1$ (checker board), $R=0.5$ (diagonal brick) and $R=0.3$ (confetti). For reference the percent change in relaxivity without spin locking (lab. frame) over the entire range of field strengths studied (red) is also shown for the same agents.

Note: the percent change by spin locking NACs at high field was calculated relative to the laboratory frame relaxaivities of the free chelate which are larger than those for NACs.

They were also found to be substantially elevated over those measured for the free chelate for which the latter shows a decreasing trend with $B_{0}$. The rotating frame relaxivities measured herein for NACs at high fields are larger anything previously reported at these fields and were found to be in agreement with our models for a theoretically "optimized" gadoterate chelate in the rotating frame. 
Laboratory frame relaxivities for NACs were found to be in agreement with our model for a theoretically "optimized" gadoterate chelate in the laboratory frame. That is, $r_{1}$ values for NACs are elevated over the free chelate at low fields, $0.47-1.41 \mathrm{~T}(20-60$ $\mathrm{MHz}$ ) but drops off dramatically at higher field strengths. At high fields, however, the laboratory frame relaxivities for NACs were found to be lower than those achievable for the free chelate. At low fields, as the charge ratio of NACs (inversely proportional to their size) decreased, the laboratory frame relaxivity was found to increase. This was consistent with previous finding for similar systems. ${ }^{93}$ However, little difference in their laboratory frame relaxivity was observed at high fields.

The use of spin locking, particularly at high fields, may prove beneficial in targeted molecular imaging applications. For such applications contrast agents are targeted to large molecular weight biomarkers giving rise to very large effective molecular weights and long $\tau_{\mathrm{R}}$ values. This work shows that employing a spin locking pulse could potentially increase relaxivities beyond values currently obtainable at high fields in the laboratory frame with comparable agents and may decrease limits of detection to levels at which biomarkers for disease pathology exist. 


\section{CHAPTER 6. EFFECT OF HYDRATION NUMBER (q) ON ROTATING FRAME LONGITUDINAL RELAXIVITY ( $\left.\boldsymbol{r}_{1}\right)$}

\subsection{Potential applications of spin locking with hydration numbers $(q) \neq 1$}

As can be seen from the SBM equation (Equation 1.19) one possible means of increasing the relaxivity of an agent is to increase its hydration number $(q)$. The hydration number refers to the number of water molecules in the inner coordination sphere of the metal ion. It is well established that by opening up extra coordination sites to additional water molecules the laboratory frame longitudinal relaxivity $\left(r_{1}\right)$ of $\mathrm{Gd}^{3+}$-based CAs scales proportionally. However, it does so at the expense of the thermodynamic stability of the chelate which has been shown to reduce by up to three orders of magnitude ${ }^{71}$ and as such all current clinical $\mathrm{Gd}^{3+}$-based CAs are restricted to just one inner sphere water molecule. However, many investigations are performed in vitro, ex vivo and/or in vivo using animal models for which the stability of the CA is a lesser concern. Moreover, for such investigations the paramagnetic metal ion is not limited to $\mathrm{Gd}^{3+}$. When looking to the periodic table for other paramagnetic ions with properties similar to $\mathrm{Gd}^{3+}, \mathrm{Mn}^{2+}$ stands out. $\mathrm{Mn}^{2+}$ has five unpaired electrons, a long electron relaxation time and can bind water molecules that undergo exchange. In fact, Lauterbur and co-workers used a salt of $\mathrm{MnCl}_{2}$ in dogs to demonstrate the importance of paramagnetic materials for MRI. ${ }^{96} \mathrm{Mn}^{2+}$ is capable of replacing the biologically active cation $\mathrm{Ca}^{2+}$ and penetrate cells ${ }^{97}$ and has found widespread use in preclinical imaging in manganese enhanced MRI (MEMRI). $\mathrm{Mn}^{2+}$ also formed the basis of two FDA approved contrast agents, Teslascan, and 
Lumenhance. ${ }^{96}$ Although $\mathrm{Mn}^{2+}$ is an important biological metal, exposure to elevated levels has been shown to have neurotoxic effects ${ }^{98}$ attributed to $\mathrm{Mn}^{2+}$ being internalized into neuronal cells. ${ }^{99-100}$ These agents have since been discontinued for clinical practice. However, there still remains a large body of work in the literature pertaining to the use of complexes of $\mathrm{Mn}^{2+}$ with various hydration numbers ex vivo and this comprises the field of MEMRI. This provided us the motivation to study the effect spin locking has on the relaxivity of agents with a variety of hydration numbers for both $\mathrm{Gd}^{3+}$ and $\mathrm{Mn}^{2+}$-based chelates.

\subsection{Low molecular weight chelates of $\mathrm{Gd}^{3+}$ and $\mathrm{Mn}^{2+}$ as systems for investigating the} effect of q on $r_{1 \rho}$

We restricted our investigations to low molecular weight complexes of $\mathrm{Gd}^{3+}$ and $\mathrm{Mn}^{2+}$ that have approximately the same rotational correlation time $\left(\tau_{R}\right)$. This allowed the effect on relaxivity of the hydration numbers of the complexes to be studied independent of the influence of $\tau_{R}$, particularly at high fields where $\tau_{\mathrm{R}}$ is non-limiting. $\mathrm{Gd}^{3+}$ and $\mathrm{Mn}^{2+}$ have nine and six coordination sites respectively and the hydration number of their complexes is dependent on the number of chelating atoms on the ligand. Chelates of $\mathrm{Gd}^{3+}$ and $\mathrm{Mn}^{2+}$ were prepared with a variety of hydration numbers (Figure 6.1) and their laboratory and rotating frame relaxivities compared over a range of low and high $B_{0}$ field strengths, 0.47 $(20 \mathrm{MHz})-14.1 \mathrm{~T}(600 \mathrm{MHz})$, at $25^{\circ} \mathrm{C}$. 
Hydration state, q

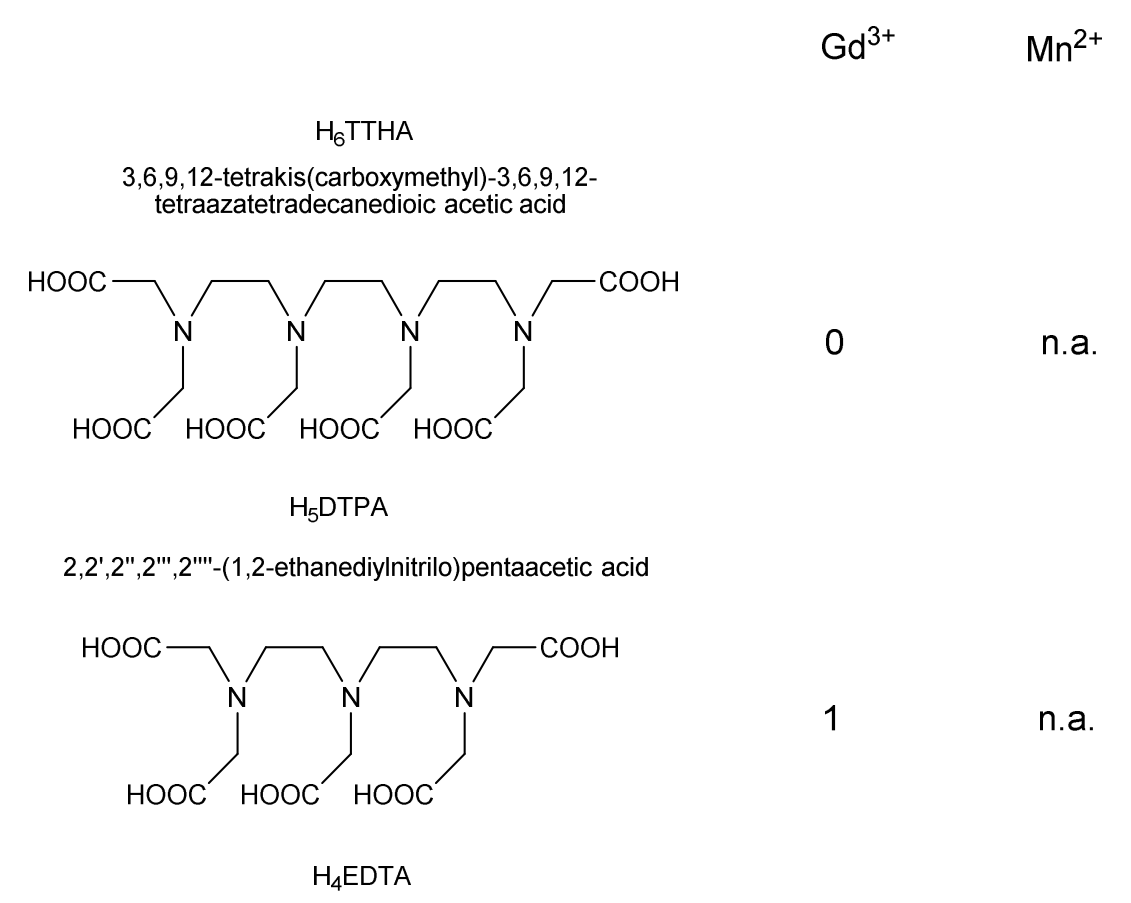

2,2',2",2"'-(1,2-ethanediyldinitrilo)tetraacetic acid

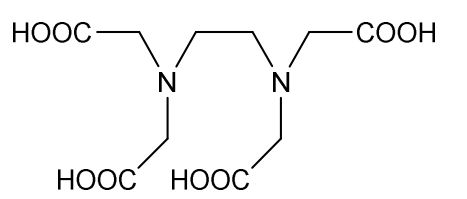

n.a. $\quad 0$

Cr

Chloride

9

6

Figure 6.1: Ligands for the preparation of low molecular weight $\mathrm{Gd}^{3+}$ and $\mathrm{Mn}^{2+}$ chelates with a variety of hydration numbers.

\subsection{Effect of $\mathcal{B B}_{1}$ on $\mathrm{R}_{1 \rho}$ for $\mathrm{MnCl}_{2}$ at low $\mathrm{B}_{0}$ field strengths}

Unlike the $\mathrm{Gd}^{3+}$ metal ion, the $\mathrm{Mn}^{2+}$ ion induces a chemical shift with characteristically broad line-widths. ${ }^{101}$ However, with no ligand field stabilization $\mathrm{Mn}^{2+}$ complexes are 
extremely labile and water exchange is rapid, leading to very large laboratory frame transverse relaxation rate constant $\left(R_{2 \mathrm{M}}\right)$. Therefore it is hypothesized that the condition invoked by the use of $\mathrm{Gd}^{3+}$ chelates, i.e. $R_{2 M} \gg \Delta \omega_{M}^{2}$, (Equation 3.3) that renders the rotating frame longitudinal relaxation rate constant $\left(R_{1 \rho}\right)$ independent of the spin locking field strength $\left(\gamma B_{1}\right)$, will hold true for $\mathrm{Mn}^{2+}$ chelates. If true, it will again allow the value of $\gamma B_{1}$ to be neglected when comparing rotating frame relaxivities of $\mathrm{Mn}^{2+}$-based CAs across several $B_{0}$ fields. Therefore, we investigated the effect of $\gamma B_{1}$ on $R_{1 \rho}$ using a $5 \mathrm{mM}$ solution of $\mathrm{MnCl}_{2}$ in $\mathrm{PBS} \mathrm{pH} 7.4$ at $20 \mathrm{MHz}(0.47 \mathrm{~T})$ and $25^{\circ} \mathrm{C}$. The strength of the spin locking field $\left(\gamma B_{1}\right)$ was modulated by attenuating the power. The Rabi frequencies expressed in $\mathrm{kHz}\left(\omega_{1}\right)$ that characterize $\gamma B_{1}$ over the attenuation range sampled corresponded to $1.1-55.4 \mathrm{kHz}$ and the results are shown in Figure 6.2.

It can clearly be seen that $R_{1 \rho}$ is unaffected across almost the entire range of Rabi frequencies sampled. Only when the $\gamma B_{1}$ is $<1.5 \mathrm{kHz}$ does the mean $R_{1 \rho}$ value begin to change, decreasing as $\gamma B_{1}$ tends to zero, which as shown by Equation 6.1, where; $R_{1 \rho}^{0}$ defines the lower power limit $\left(\omega_{1} \rightarrow 0\right)$ effect on $R_{1 \rho}$ - again from the work of Jordan and co-workers, ${ }^{68}$ but also explained by others ${ }^{63,102}$ - and should tend to $R_{2}$ values.

$$
R_{1 \rho}^{0}=R_{2 W}+\frac{q[M]}{55.6} \frac{1}{\tau_{M}}\left\{\frac{R_{2 m}\left(R_{2 M}+1 / \tau_{M}\right)+\Delta \omega_{m}^{2}}{\left(R_{2 M}+1 / \tau_{M}\right)^{2}+\Delta \omega_{m}^{2}}\right\}=R_{2}
$$

It is noteworthy that in comparison to the data for the $\mathrm{Gd}^{3+}$-based CAs at $20 \mathrm{MHz}$ and 25 ${ }^{\circ} \mathrm{C}$ (Figure 3.1a and 3.1c for ProHance and Magnevist respectively) the data for $\mathrm{MnCl}_{2}$ 
have much lower associated errors, and effective spin locking occurred at lower powers. The reduced spin lock field strength required to effectively lock the spins of $\mathrm{MnCl}_{2}$ is a consequence of narrower line-widths for the signal, a result of a much lower sample concentration employed due to the potency of $\mathrm{MnCl}_{2}$ at effecting relaxation rates.

It follows from these experiments that in order to generate accurate and precise $R_{1 \rho}$ data, useful for creating NMRD profiles, effective spin locking must occur. From this data this appears to occur with a minimum spin locking field strength $\left(\gamma B_{1}\right)$ of $\sim 5 \mathrm{kHz}$, again an unrealistic value as a consequence of the likely incorrect pulse duration obtained from the minispec instrument, vide supra.

Under conditions of effective spin locking, the rotating frame relaxivity of $\mathrm{Mn}^{2+}$-based chelates is largely independent of the strength of the spin locking field, as was the case for $\mathrm{Gd}^{3+}$-based CAs. This suggests that the $\mathrm{Mn}^{2+}$-bound water laboratory frame transverse relaxation rate constant must therefore be significantly larger than the chemical shift difference between the metal bound and bulk water protons induced by $\mathrm{Mn}^{2+}$ (i.e. $\mathrm{R}_{2 \mathrm{M}} \gg \Delta \omega_{M}^{2}$ ). As such, this allows for the comparison of rotating frame longitudinal relaxivities of $\mathrm{Mn}^{2+}$-based chelates across several $B_{0}$ fields without consideration of the effect of the spin locking pulse strength. 


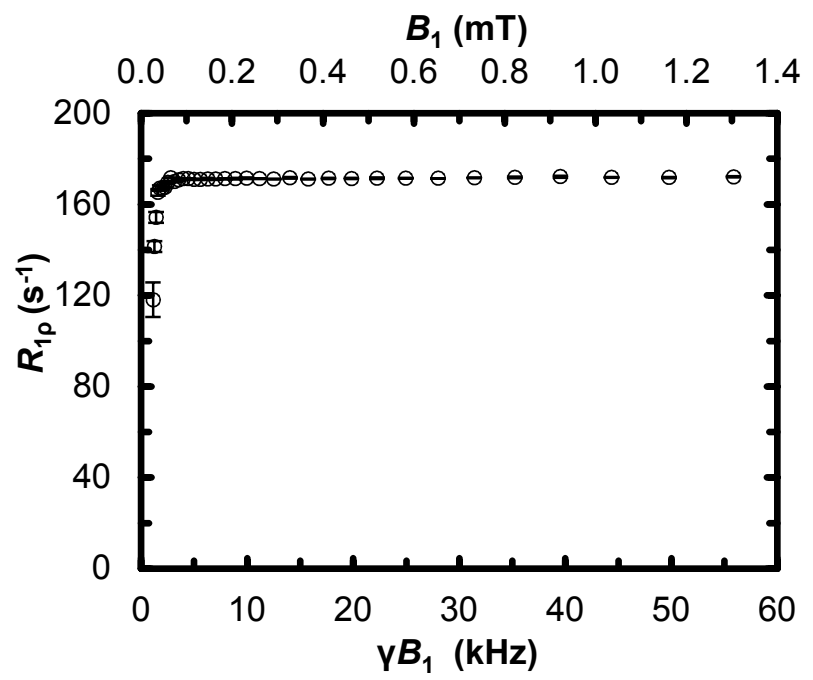

Figure 6.2: The dependence of the water proton rotating frame longitudinal relaxation rate constant $\left(R_{1 \mathrm{p}}\right)$ on the strength of the spin locking field $\left(\gamma B_{1}\right)$ for a $5 \mathrm{mM} \mathrm{MnCl}_{2}$ solution in PBS at $25^{\circ} \mathrm{C}$ and $20 \mathrm{MHz}$. Data shown are the mean of six measurements; the error bars represent the SD of the data. Note: $\gamma B_{1}$ values calculated from a $90^{\circ}$ pulse duration reported from the minispec instrument. These values are surprisingly large and suggest the the pulse duration reported is incorrect.

\subsection{A comparison of laboratory $\left(r_{1}\right)$ and rotating ( $\left.r_{1 \rho}\right)$ frame NMRD for low molecular weight $\mathrm{Gd}^{3+}$ and $\mathrm{Mn}^{2+}$ agents as a function of hydration number (q)}

Laboratory $\left(r_{1}\right)$ and rotating $\left(r_{1}\right)$ frame relaxivities for low molecular weight chelates of $\mathrm{Gd}^{3+}$ and $\mathrm{Mn}^{2+}$ with a variety of hydration number prepared in this study were determined over a range of $B_{0}$ field strengths and their NMRD profiles generated.

6.4.1. A comparison of $r_{1}$ and $r_{1 \rho}$ for low molecular weight non-bulky $G d^{3+}$ complexes

The laboratory frame relaxivities of $\operatorname{GdTTHA}^{3-}(\mathrm{q}=0)$, $\operatorname{GdDTPA}(\mathrm{q}=1)$ and $\mathrm{GdCl}_{3}(\mathrm{q}=$ 9) follow a similar trend to those observed for clinical $\mathrm{Gd}^{3+}$-based CAs (Figure 6.3) in 
that $r_{1}$ decreases as $B_{0}$ increases, and increases as the hydration number increases. By applying a spin lock pulse, rotating frame relaxivities were again larger than the laboratory frame relaxivities at all field strengths measured $(0.47-14.1 \mathrm{~T}, 20-600$ $\mathrm{MHz}$ ), increased as a function of increasing hydration number and decreased as $B_{0}$ increased. It was observed for all three agents that, again, the rotating frame relaxivity decreased slightly less rapidly than in the laboratory frame. As with clinical agents, the gain in relaxivity for each chelate by employing a spin locking pulse is fairly modest, showing an increase in relaxivity by a factor of just 1.10 at $0.47 \mathrm{~T}$ for $\mathrm{GdTTHA}^{3-}$, a $\mathrm{q}=0$ complex, rising to 1.35 at $14.1 \mathrm{~T}$ for GdDTPA, a $\mathrm{q}=1$ complex (Table 6.1). The magnitude of the increase in relaxivity by applying a spin locking pulse is again not necessarily proportional to the magnitude of the laboratory frame relaxivity. This can be seen from the data for the $q=9$ chelate $\mathrm{GdCl}_{3}$ at $14.1 \mathrm{~T}$ which increases from 10.7 to 13.1 $\mathrm{mM}^{-1} \mathrm{~s}^{-1}$ a factor of only 1.22 compared to 1.35 for GdDTPA (Table 6.1). Moreover, as predicted by SBM theory (Equation 1.9), at all field strengths studied the laboratory frame relaxivity increases as the hydration number increases. This was also observed for the rotating frame relaxivities.

\subsubsection{A comparison of $r_{1}$ and $r_{1 \rho}$ for low molecular weight $\mathrm{Mn}^{2+}$ complexes}

The laboratory frame relaxivities of $\operatorname{MnEDTA}(\mathrm{q}=0)$ and $\mathrm{MnCl}_{2}(\mathrm{q}=6)$ follow a similar trend to those observed for $\mathrm{Gd}^{3+}$ complexes (Figure 6.4) in that $r_{1}$ decreases as $B_{0}$ increases, and increases as the hydration number increases. By applying a spin lock pulse, rotating frame relaxivities were again larger than the laboratory frame relaxivities 
at all field strengths measured $(0.47-14.1 \mathrm{~T}, 20-600 \mathrm{MHz})$ and also increased as a function of increasing hydration number. However, the relaxivities in the rotating frame for both $\mathrm{Mn}^{2+}$ complexes increase on moving to higher $B_{0}$ fields rather than decrease as for $r_{1}$ and the $\mathrm{Gd}^{3+}$ analogues. By applying a spin lock pulse the relaxivity of MnEDTA, a $\mathrm{q}=0$ complex, increases from $4.07 \mathrm{mM}^{-1} \mathrm{~s}^{-1}$ to $13.60 \mathrm{mM}^{-1} \mathrm{~s}^{-1}$ at $0.47 \mathrm{~T}$ and $25{ }^{\circ} \mathrm{C}$ (a factor of 3.34).

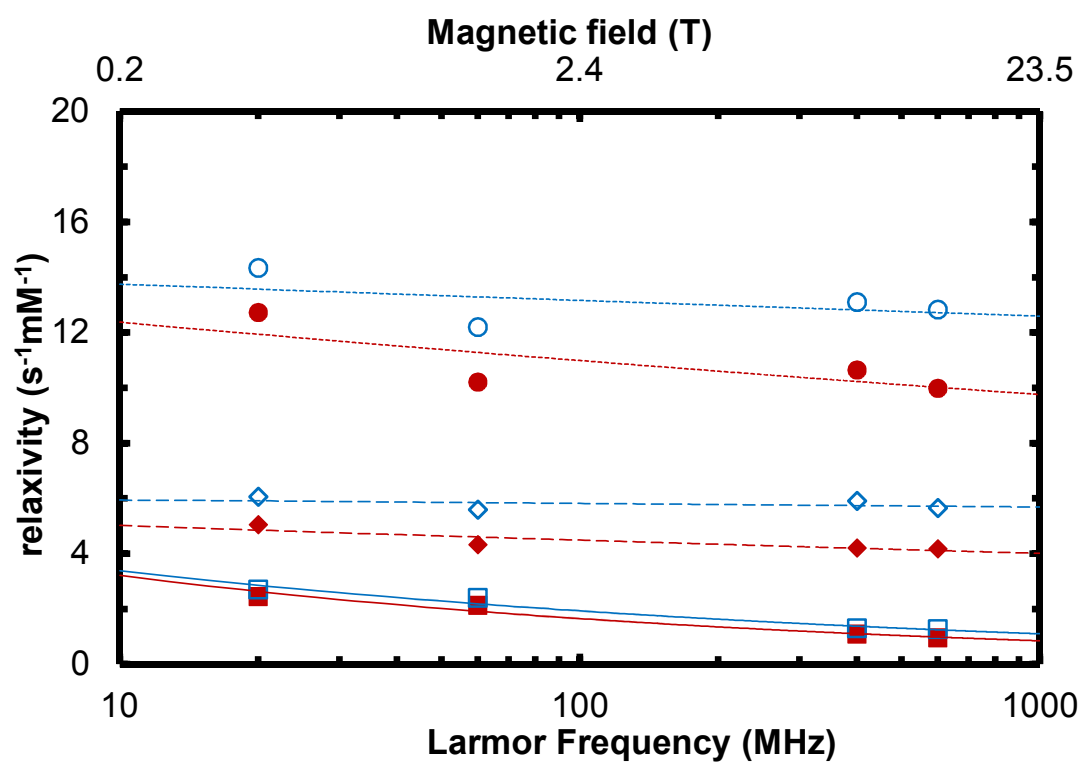

Figure 6.3: NMRD profiles of low molecular weight chelates of $\mathrm{Gd}^{3+}$ with varying hydration numbers studied herein at $25{ }^{\circ} \mathrm{C}$ in $\mathrm{H}_{2} \mathrm{O}$ : laboratory frame data $\left(r_{1}\right)$ are presented in red (closed symbols); and rotating frame data $\left(r_{1}\right)$ are presented in blue (open symbols); $\omega_{1}=35 \mathrm{kHz}(0.47$ and $1.41 \mathrm{~T}$ ) and 6924.2 and $17237.0 \mathrm{~Hz}\left(9.4\right.$ and $14.1 \mathrm{~T}$ respectively). GdTTHA ${ }^{3-}, \mathrm{q}=0$, (squares); GdDTPA, $q=1$, (diamonds); $\mathrm{GdCl}_{3}, \mathrm{q}=9$, (circles). Lines through the data are to guide the eye only. Data shown are the mean of six measurements; error bars representing the $\mathrm{SD}$ of the data were omitted for clarity, all were smaller than the markers. 
Table 6.1: Raw $r_{1}$ and $r_{1 \rho}$ data at $25^{\circ} \mathrm{C}$ used to generate the NMRD above for the low molecular weight non-bulky Gd ${ }^{3+}$ complexes studied herein at low and high field and their calculated ratios. Mean represents the average difference for the specified category of agents.

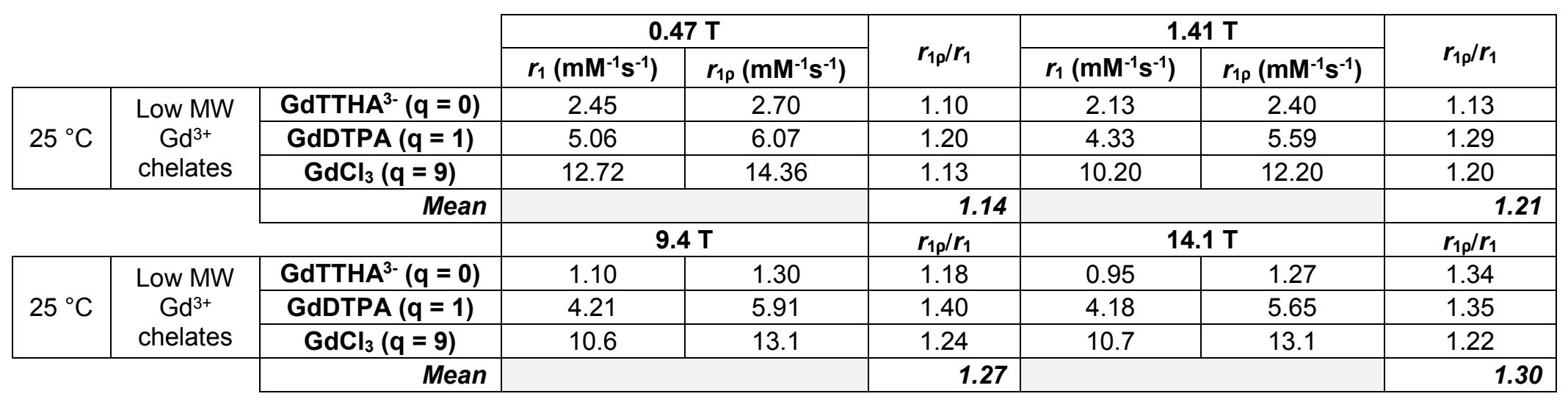


At $14.1 \mathrm{~T}$ and $25{ }^{\circ} \mathrm{C}$ the increase is from $3.04 \mathrm{mM}^{-1} \mathrm{~s}^{-1}$ to $41.3 \mathrm{mM}^{-1} \mathrm{~s}^{-1}$ (a factor of 13.6) (Table 6.2). More substantial gains in relaxivity were observed for the $q=6 \mathrm{MnCl}_{2}$ complex. At $0.47 \mathrm{~T}$ and $25{ }^{\circ} \mathrm{C}$ the relaxivity increased from $6.85 \mathrm{mM}^{-1} \mathrm{~s}^{-1}$ in the laboratory frame to $38.26 \mathrm{mM}^{-1} \mathrm{~s}^{-1}$ in the rotating frame (a factor of 5.59). But, at $14.1 \mathrm{~T}$ and $25^{\circ} \mathrm{C}$ the increases was a factor of 31.1 going from $4.99 \mathrm{mM}^{-1} \mathrm{~s}^{-1}$ in the laboratory frame to $155 \mathrm{mM}^{-1} \mathrm{~s}^{-1}$ in the rotating frame! This is by far the highest relaxivity ever observed for a discrete metal complex at such a high magnetic field. Like clinical $\mathrm{Gd}^{3+}$ based $\mathrm{CA}$, these complexes are in the fast exchange regime. As such the relaxivities of $\mathrm{MnCl}_{2}$, for example, decrease when the temperature is increased.

In 1984 Keonig and Brown reviewed the implications for NMR imaging (MRI) of solvent proton relaxation by paramagnetic solutes and its dependence on magnetic field and chemical environment. ${ }^{103}$ In that paper the dependence on magnetic field of $1 / T_{1}\left(R_{1}\right)$ and $1 / T_{2}\left(R_{2}\right)$ of water protons for aqueous solutions of several transition metal ions, including $\mathrm{Mn}^{2+}$, were determined (Figure 6.5). SBM theory that explains the field dependence of $1 / T_{1}$ can also be applied to describe $1 / T_{2}$. The normalized $r_{1}$ and $r_{2}$ NMRD profiles for $\mathrm{Mn}^{2+}$ were shown to be unique from each other and also amongst the other ions analyzed. At low fields $(<\sim 1 \mathrm{MHz}) r_{1}$ and $r_{2}$ values for all ions except $\mathrm{Mn}^{2+}$ are essentially independent of field strength and follow the same trend, however, for $\mathrm{Mn}^{2+}$ ions an inflection at this point is observed with the relaxivities increasing substantially as $B_{0}$ decreases towards $0.01 \mathrm{MHz}$. 


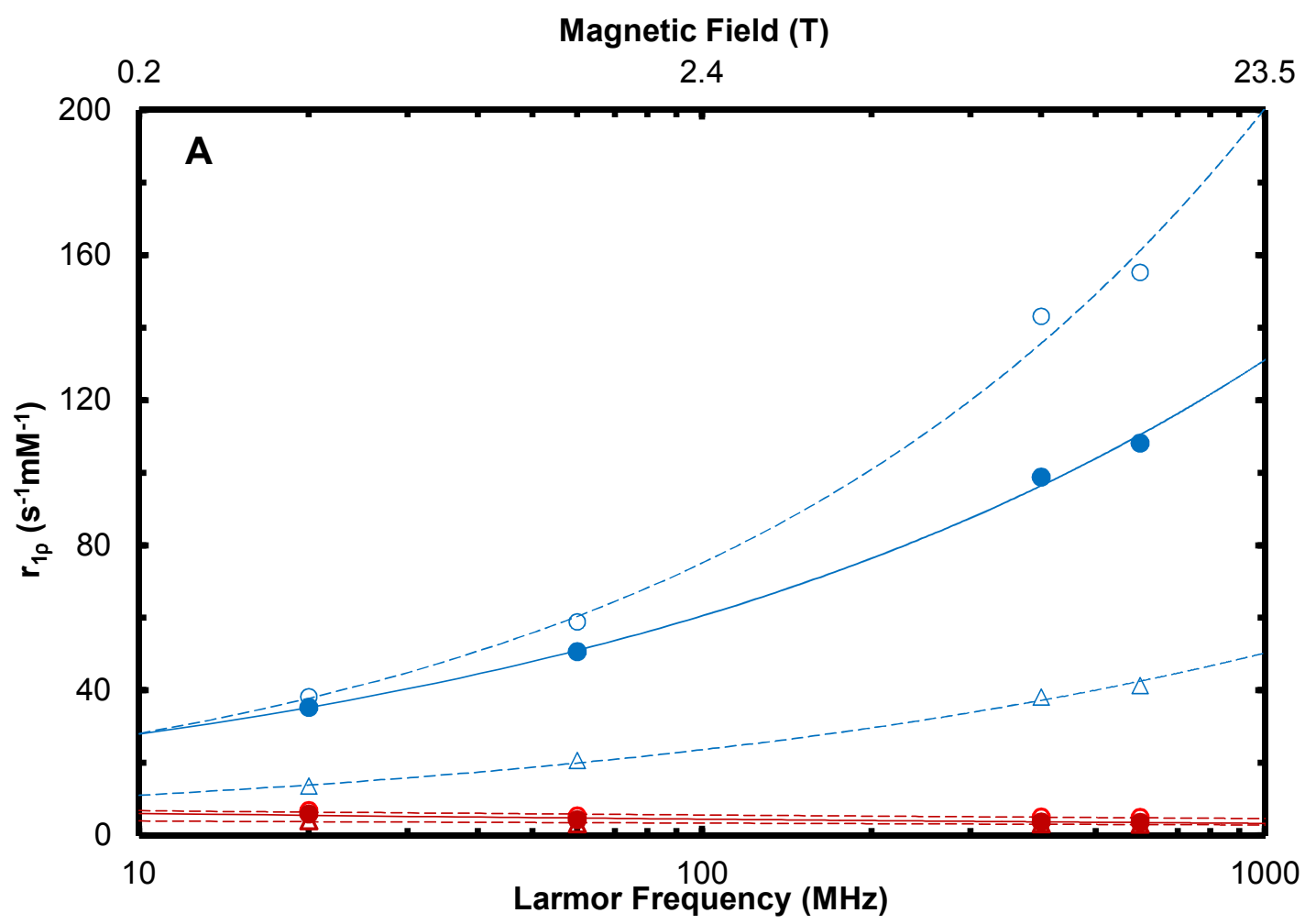

Figure 6.4: NMRD profiles of low molecular weight $\mathrm{Mn}^{2+}$ chelates with varying hydration numbers, MnEDTA (triangles) $=0, \mathrm{MnCl}_{2}$ (circles) $=6$, studied herein at $25^{\circ} \mathrm{C}$ (open symbols) and $37^{\circ} \mathrm{C}$ (closed symbols) in $\mathrm{H}_{2} \mathrm{O}$. Laboratory $\left(r_{1}\right)$ and rotating frame $\left(r_{1 p}\right)$ frame data are presented in red and blue respectively. $\omega_{1}=35 \mathrm{kHz}(0.47$ and $1.41 \mathrm{~T})$ and 6924.2 and 17237.0 $\mathrm{Hz}$ (9.4 and 14.1 T respectively). Lines through the data are to guide the eye only. Data shown are the mean of six measurements; error bars representing the SD of the data were omitted for clarity, all were smaller than the markers.

At high fields the trend in $r_{1}$ for all ions, including $\mathrm{Mn}^{2+}$, are comparable and differ only by the ratio $T_{1} / T_{2}=3.5 / 3$. An inflection occurs at around $3 \mathrm{MHz}$ and the relaxivities decrease as $B_{0}$ increases. As $B_{0}$ increases up to $\sim 10 \mathrm{MHz}, r_{2}$ for $\mathrm{Mn}^{2+}$ follows the same trend as for $r_{1}$, albeit with larger values. However, at higher $B_{0}$ values $r_{2}$ rapidly and substantially increases. 
CHAPTER 6. Effect of $q$ on $r_{1} \rho$

Table 6.2: Raw $r_{1}$ and $r_{1 p}$ data at 25 and $37^{\circ} \mathrm{C}$ used to generate the NMRD above for the low molecular weight $\mathrm{Mn}^{2+}$ complexes studied herein at low and high field and their calculated ratios. 
Unlike the other transition metal ions studied, and $\mathrm{Gd}^{3+}$ ions/chelates, $\mathrm{Mn}^{2+}$ has a large contribution to relaxation from scalar coupling. ${ }^{104}$ This is a result of a widely delocalized electronic wave function for hydrated $\mathrm{Mn}^{2+}$ ions such that the electron spin momenta has a greater interaction with the bound water protons. Although the scalar interaction is small compared to the dipole-dipole interaction, the contribution to the NMRD profile is large due to a 100-fold difference in their correlation times. The characteristic $r_{1}$ and $r_{2}$ NMRD profiles for $\mathrm{Mn}^{2+}$ ions are a consequence of this same phenomena.

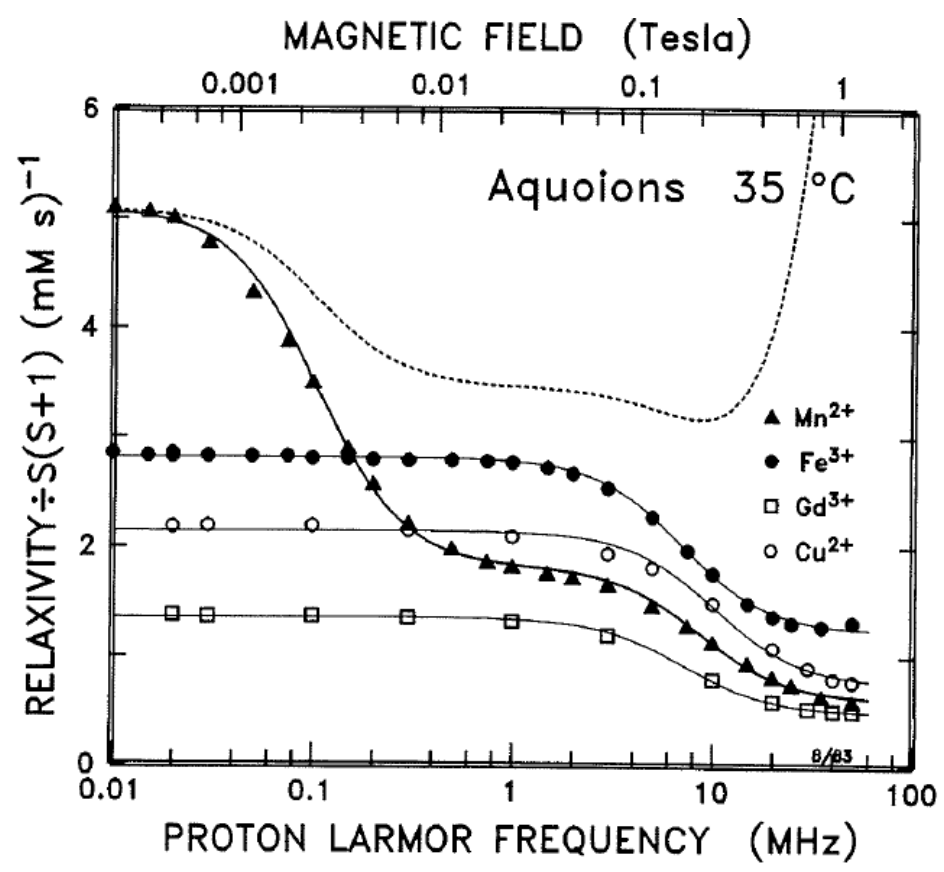

Figure 6.5: $r_{1}$ NMRD profiles of aqueous soultions of transition metal ions at $35^{\circ} \mathrm{C}$ scaled by the value of $S(S+1)$ where $S$ is their spin quantum number. The solid lines through the data are a result of a least-squares fitting of the data with well established theory appropriate to the solutions. $r_{2}$ profiles are not shown for any other ion except $\mathrm{Mn}^{2+}$ since they would be similar to $r_{1}$. $\mathrm{Mn}^{2+} r_{2}$ values (dotted line) were derived from theory and supported by experiment. 
Since a spin locking pulse incorporates a component of laboratory frame transverse relaxation $\left(R_{2 \mathrm{M}}\right)$ that is especially sensitive to the scalar contribution as described above this explains the exceptionally high rotating frame relaxivities obtained herein for our $\mathrm{Mn}^{2+}$ chelates.

\subsection{Conclusions}

Only modest gains in relaxivity were measured for the low molecular weight $\mathrm{Gd}^{3+}$ chelates by spin locking and the values decrease as $B_{0}$ increases as has been observed previously herein for the structurally similar clinical $\mathrm{Gd}^{3+}$-based CAs. However, very large gains were observed for $\mathrm{Mn}^{2+}$ agents at all fields and increases as $B_{0}$ increases due to their unique magnetic properties. Moreover, the results demonstrate that relaxivity in the rotating frame scales proportionally with $\mathrm{q}$. We demonstrated that in the rotating frame relaxivity lost on moving to higher $B_{0}$ field strengths was able to be "recovered" across all $B_{0}$ field strengths studied and for all hydration numbers (q) of $\mathrm{Gd}^{3+}$ and $\mathrm{Mn}^{2+}$ studied.

It has clearly been demonstrated that by combining the spin locking technique with such $\mathrm{Mn}^{2+}$ agents the potential exists to increase detection limits dramatically. This approach may prove to be most useful in ex vivo or in vivo animal model studies where the known stability and toxicity problems associated with these agents is a lesser concern. Such experiments would contribute to the field of Manganese Enhanced MRI (MEMRI), a growing area of research accelerated by recent safety concerns of clinical $\mathrm{Gd}^{3+}$-based agents. ${ }^{105-106}$ 


\section{CHAPTER 7. EXPERIMENTAL SECTION}

\subsection{Reagents and solvents}

All reagents were purchased from commercially available sources unless otherwise noted. Type I water $(18.2 \mathrm{MOhm} \cdot \mathrm{cm})$ was used for the preparation of all solutions of contrast media and phosphate buffered saline. Phosphate buffered saline (PBS) pH 7.4 (50 $\mathrm{mM}$ phosphate buffer with $15 \mathrm{mM} \mathrm{NaCl}$ ) was prepared by combining $1 \mathrm{M}$ aqueous sodium dihydrogenorthophosphate solution $(7.6 \mathrm{~mL}), 1 \mathrm{M}$ aqueous disodium hydrogenorthophosphate heptahydrate solution $(42.4 \mathrm{~mL})$ and $5 \mathrm{M}$ aqueous sodium chloride solution $(30.0 \mathrm{~mL})$ and diluting to $2 \mathrm{~L}$ with water.

\subsection{Instrumentation}

Relaxation time constants were determined at low field using Bruker Minispec desktop relaxometers operating at fixed fields of $20 \mathrm{MHz}(0.47 \mathrm{~T})$ and $60 \mathrm{MHz}(1.41 \mathrm{~T})$ using a 2 $\mathrm{mm}$ broad-band probe with the temperature controlled using the installed Julabo F25-ED variable-temperature controller. High field relaxation time constants were determined using Bruker Avance IIa and III NMR spectrometers operating at $400.13 \mathrm{MHz}(9.4 \mathrm{~T})$ and 599.92 MHz (14.1 T), respectively. Both high field spectrometers were installed with $5 \mathrm{~mm}$ broad-band probes and the temperature was controlled using the installed variable-temperature controller model 2416 with BCU-05 chiller. 


\subsection{Pulse sequences}

\subsubsection{Inversion recovery pulse sequence}

The factory installed inversion recovery pulse sequence was used throughout, this comprises: $180^{\circ}$ inversion pulse $-\tau$ variable pulse delay $-90^{\circ}$ excitation pulse acquisition of FID.

\subsubsection{Carr-Purcell-Meiboom-Gill (CPMG) pulse sequence}

The factory installed CPMG pulse sequence was used throughout, this comprises: $90^{\circ}$ excitation pulse $-\tau$ variable pulse delay $-180^{\circ}$ (n) refocusing pulses - acquisition of FID.

\subsubsection{Spin locking pulse sequence}

The factory installed spin locking pulse sequence was used throughout, this comprises: $90^{\circ}$ excitation pulse $-\tau$ variable spin locking pulse duration - acquisition of FID.

\subsection{Fitting}

All rate constant data acquired at 0.47 and $1.41 \mathrm{~T}$ were analyzed using the factory installed fitting routine. All rate constant data acquired at 9.4 and $14.1 \mathrm{~T}$ were fitted offline using a least squares fitting routine in Excel to Equations 1.12 ( $T_{1}$ data), 1.13 ( $T_{2}$ data) and $1.24\left(T_{1 \rho}\right)$. 


\subsection{Contrast media stock solution preparations}

\subsubsection{Clinical $\mathrm{Gd}^{3+}$-based contrast agents}

Seven of the nine clinically available $\mathrm{Gd}^{3+}$-based contrast agents were studied. Three were macrocyclic chelates: gadoterate meglumine (Dotarem, $500 \mathrm{mM}$ ), gadoteridol (ProHance, $500 \mathrm{mM}$ ), gadobutrol (Gadavist, $1000 \mathrm{mM}$ ). Four were linear chelates: of which two were low molecular weight: gadopentetate dimeglumine (Magnevist, 500 $\mathrm{mM}$ ) and gadodiamide (Omniscan, $500 \mathrm{mM}$ ); and two incorporated bulky substituents: gadobenate dimeglumine (MultiHance, $500 \mathrm{mM}$ ) and gadofosveset trisodium (Ablavar, $250 \mathrm{mM}$ ). All were obtained and studied in their clinical formulations and used as stock solutions. Gadoversetamide (Optimark) and gadoxetate disodium (Eovist/Primovist) were not studied given the structural similarities between these agents and gadodiamide (Omniscan) and gadobenate (MultiHance), respectively and the likelihood of identical or similar results for these two pairs. All contrast media were purchased from commercial sources with the exception of gadoterate which was a gift from Guerbet s.a.

\subsection{2 $\mathrm{GdCl}_{3}$}

Approximately $5 \mathrm{~mL}$ of concentrated $\mathrm{HCl}(12.1 \mathrm{M})$ was added to a small quantity of $\mathrm{Gd}_{2} \mathrm{O}_{3}$ to dissolve. The solvent was evaporated by heating at $60-80{ }^{\circ} \mathrm{C}$ with stirring for several hours. Several subsequent small aliquots $(1-2 \mathrm{~mL})$ of $\mathrm{HCl}(12.1 \mathrm{M})$ were added and solvent evaporated in the same manner. After the final evaporation of solvent a 
white crystalline product of $\mathrm{GdCl}_{3}$ was afforded and used 'as is'. A stock solution of $\mathrm{GdCl}_{3}(109.5 \mathrm{mM})$ was prepared by dissolving $\mathrm{GdCl}_{3}(577.0 \mathrm{mg})$ in water $(20 \mathrm{~mL})$.

\subsection{3. $\mathrm{MnCl}_{2}$}

A stock solution of $\mathrm{MnCl}_{2}(\sim 120 \mathrm{mM})$ was prepared by dissolving $\mathrm{MnCl}_{2}(0.72 \mathrm{mmol}$, $0.091 \mathrm{~g})$ in water $(6 \mathrm{~mL})$. The actual $\mathrm{MnCl}_{2}$ concentration of this solution was determined by relaxometry $\left(20 \mathrm{MHz}\right.$ and $\left.25{ }^{\circ} \mathrm{C}\right)$. For this, the laboratory frame longitudinal relaxation rate constant for the stock solution and a series of dilute aqueous solutions were determined. Linear regression analysis of the experimental data using the least squares method and the known relaxivity for this agent under similar conditions $\left(7.44 \mathrm{mM}^{-1} \mathrm{~s}^{-1}\right)^{103}$ using the solver function in Excel afforded the actual concentration of the stock solution $(131.3 \mathrm{mM})$.

\subsubsection{GdTTHA $A^{3-}$}

A stock solution of $\mathrm{GdTTHA}^{3-}(\sim 50 \mathrm{mM})$ was prepared by dissolving $\mathrm{GdCl}_{3}(0.5161$ mmol, $0.1918 \mathrm{~g})$ and $\mathrm{H}_{6}$ TTHA $(0.5485 \mathrm{mmol}, 0.2712 \mathrm{~g})$ in a small volume of water $(\sim 3$ $\mathrm{mL}$ ), adjusting the $\mathrm{pH}$ to $\sim 5$ with dilute solutions of $\mathrm{NaOH} / \mathrm{HCl}$ and diluting to $\sim 20 \mathrm{~mL}$ with water prior to freeze drying the solution and re-dissolving the GdTTHA ${ }^{3-}$ in PBS $\mathrm{pH}$ $7.4(10 \mathrm{~mL})$. The actual GdTTHA ${ }^{3-}$ concentration of this solution was determined by relaxometry $\left(20 \mathrm{MHz}\right.$ and $\left.25^{\circ} \mathrm{C}\right)$. For this, the laboratory frame longitudinal relaxation rate constant for a series of dilute solutions of GdTTHA ${ }^{3-}$ in PBS pH 7.4 were determined. Linear regression analysis of the experimental data using the least squares 
method and the known relaxivity for this agent under identical conditions $\left(1.7 \mathrm{mM}^{-1} \mathrm{~s}^{-}\right.$ $\left.{ }^{1}\right)^{107}$ using the solver function in Excel afforded the actual concentration of the stock solution $(31.31 \mathrm{mM})$.

\subsubsection{MnEDTA}

An aqueous stock solution of MnEDTA $(56.8 \mathrm{mM})$ was prepared by mixing $\mathrm{MnCl}_{2}$ stock solution ( $3.0 \mathrm{~mL}, 0.39 \mathrm{mmol})$ and $100 \mathrm{mM} \mathrm{Na} 2 \operatorname{EDTA}(3.938 \mathrm{~mL}, 0.39 \mathrm{mmol})$.

\subsection{Working solution preparations and experimental parameters}

7.6.1. Chapter 2. Effect of pulse sequence parameters on longitudinal $\left(T_{1}\right)$ and rotating $\left(T_{1 \rho}\right)$ frame time constants

For the purposes of determining the effect pulse sequence parameters have on laboratory and rotating frame relaxation time constants ( $T_{1}$ and $T_{1 \rho}$ respectively) a solution $(25 \mathrm{mM})$ of Dotarem was prepared from the formulated product. Experiments were performed at 25 and $37^{\circ} \mathrm{C}$ using a low fixed field Bruker Minispec desktop relaxometer operating at $60 \mathrm{MHz}(1.41 \mathrm{~T})$. The factory installed inversion recovery and spin locking pulse sequences were employed. Except where changed for the purpose of the experiment the following pulse sequence parameters were used; inversion recovery pulse sequence; recycle delay $=0.1 \mathrm{~s}$; number of data points $=16$; number of averages $=4$ and the inversion pulse delay times ranged from $0.2-100 \mathrm{~ms}$. Spin locking pulse sequence; recycle delay $=4 \mathrm{~s}$; number of data points $=16$; number of averages $=4$; spin locking 
pulse durations ranged from 2 to $40 \mathrm{~ms}$ and the Rabi frequency $\left(\omega_{1}\right)=20 \mathrm{kHz}$. Relaxation time constants were obtained as single measurement determinations.

\subsubsection{Chapter 3. Effect of spin lock field strength $\left(\gamma B_{1}\right)$ on the rotating frame} longitudinal relaxation rate constant $\left(R_{1 \rho}\right)$

For the purposes of $B_{1}$ power experiments the rotating frame longitudinal relaxation rate constants $\left(R_{1 \rho}\right)$ were determined for $100 \mathrm{mM}$ solutions of ProHance and Magnevist in PBS (0.47 and $1.41 \mathrm{~T})$ and a $5 \mathrm{mM}$ solution of Dotarem in PBS (14.1 T) prepared from the respective formulated products. The factory installed spin locking pulse sequence was employed with the following pulse sequence parameters; recycle delay $=4 \mathrm{~s}$; number of data points $=16$; number of averages $=4$; spin locking pulse durations ranged from $2-$ $20 \mathrm{~ms}(0.47$ and $1.41 \mathrm{~T})$ and 25 to $800 \mathrm{~ms}(14.1 \mathrm{~T})$. The Rabi frequency $\left(\omega_{1}\right)$ was varied from $1.2 \mathrm{kHz}$ to $55.4 \mathrm{kHz}(0.47$ and $1.41 \mathrm{~T})$ and $209.6 \mathrm{~Hz}$ to $17237 \mathrm{~Hz}(14.1 \mathrm{~T})$. Relaxation time constants were determined by averaging the values obtained from six measurements for each value of $\omega_{1}$.

7.6.3. Chapter 4. A comparison of laboratory $\left(r_{1}\right)$ and rotating $\left(r_{1 \rho}\right)$ frame longitudinal relaxivities for $q=1$ clinical $G d^{3+}$-based CAs

NMRD profiles were generated from laboratory and rotating frame relaxivity determinations of each clinically approved agent (Dotarem, ProHance, Gadavist, Magnevist, Omniscan, MultiHance and Ablavar) in PBS at the four magnetic field strengths studied $(0.47,1.41,9.4$ and $14.1 \mathrm{~T})$. Relaxivities were determined by linear 
regression analysis of the longitudinal relaxation rate constant as a function of contrast agent concentration in Excel.

Rotating frame longitudinal relaxation rate constants $\left(R_{1 \rho}\right)$ were determined on five solutions of each contrast agent in PBS varying in concentration from 10 to $25 \mathrm{mM}(0.47$ and $1.41 \mathrm{~T})$ and 5 to $25 \mathrm{mM}(9.4$ and $14.1 \mathrm{~T})$ with the exception of Dotarem at 600.13 $\mathrm{MHz}$ which was measured on five solutions varying in concentration from 1 to $5 \mathrm{mM}$ solutions. The factory installed spin locking pulse sequence was employed with the following pulse sequence parameters; recycle delay $\geq 10 \times T_{1}$; number of data points $=$ 16 ; number of averages $=4$; spin locking pulse durations ranged from 2 to $40 \mathrm{~ms}(0.47$ and $1.41 \mathrm{~T})$ and $2.4-1600 \mathrm{~ms}(9.4$ and $14.1 \mathrm{~T})$ and the Rabi frequency $\left(\omega_{1}\right)=35 \mathrm{kHz}$ (0.47 and $1.41 \mathrm{~T}$ ) and 6924.2 and $17237.0 \mathrm{kHz}$ (9.4 and $14.1 \mathrm{~T}$ respectively).

For purposes of generating laboratory frame NMRD profiles longitudinal relaxation rate constants $\left(R_{1}\right)$ were determined using five solutions varying in concentration from 1 to 5 $\mathrm{mM}$ solutions of each contrast agent in PBS. The factory installed inversion recovery pulse sequence was used with the following parameters: Recycle delay $\geq 10 \times T_{1}$; number of data points $=16$; number of averages $=4$; inversion pulse delay times ranged from 2 $\mathrm{ms}$ to $2500 \mathrm{~ms}(0.47$ and $1.41 \mathrm{~T})$ and $10 \mathrm{~ms}$ to $5000 \mathrm{~ms}(9.4$ and $14.1 \mathrm{~T})$. Relaxation time constants were determined by averaging the values obtained from six measurements for each concentration. 
7.6.4. Chapter 5. Effect of rotational correlation time $\left(\tau_{R}\right)$ on rotating frame longitudinal relaxivity $\left(r_{1 \rho}\right)$

NMRD profiles were generated from laboratory frame relaxivity determinations of nanoassembled capsules (NACs) prepared at charge ratios (R) of $0.3,0.5$ and 1 in water at the four magnetic field strengths studied $(0.47,1.41,9.4$ and $14.1 \mathrm{~T}, \mathrm{R}=0.3$ only performed at $0.47 \mathrm{~T}$ ). Rotating frame relaxivity determination for the same samples could only be determined at 9.4 and $14.1 \mathrm{~T}(\mathrm{R}=0.3$ only performed at $14.1 \mathrm{~T})$ due to instrument limitations which limits the maximum spin lock pulse duration to $40 \mathrm{~ms}$, insufficient time to observe complete relaxation with the sample concentration available. NMRD profiles were also generated from the laboratory and rotating frame relaxivity determinations of the free GdDOTP ${ }^{5-}$ chelate in water at the four magnetic field strengths studied $(0.47,1.41,9.4$ and $14.1 \mathrm{~T})$. Relaxivities were determined by linear regression analysis of the longitudinal relaxation rate constant as a function of $\mathrm{Gd}^{3+}$ concentration in Excel. The aqueous suspension of NACs was mixed thoroughly in the NMR sample tube prior to each experiment and allowed to equilibrate to the set sample temperature for at least 5 minutes. This was repeated for each repetition to minimize errors in the measurement due to settling of the NACs in the tube.

Rotating frame longitudinal relaxation rate constants $\left(R_{1 \rho}\right)$ for NACs were determined on four solutions in water. The concentrations varied between $0.028-0.98 \mathrm{mM}(9.4$ and 14.1 T). 
For the free chelate five solutions were used varying in concentration from $10-25 \mathrm{mM}$ $(0.47$ and $1.41 \mathrm{~T})$ and $1-5(9.4$ and $14.1 \mathrm{~T})$. The factory installed spin locking pulse sequence was employed with the following pulse sequence parameters; recycle delay $\geq$ $10 \times T_{1}$; number of data points $=16$; number of averages $=4$; spin locking pulse durations for GdDOTP ${ }^{5-}$ ranged from $2-40 \mathrm{~ms}$ with a Rabi frequency $\left(\omega_{1}\right)=35 \mathrm{kHz}$ $(0.47$ and $1.41 \mathrm{~T})$ and $5-1500 \mathrm{~ms}$ with a Rabi frequency $\left(\omega_{1}\right)=6924.2$ and $17237.0 \mathrm{kHz}$ (9.4 and 14.1 T respectively). For NACs the spin locking pulse durations ranged from 4 - $400 \mathrm{~ms}$ with a Rabi frequency $\left(\omega_{1}\right)=6924.2$ and $17237.0 \mathrm{kHz}(9.4$ and $14.1 \mathrm{~T}$ respectively). Relaxation time constants were determined by averaging the values obtained from three measurements for each concentration.

Laboratory frame longitudinal relaxation rate constants $\left(R_{1}\right)$ for NACs were determined on four solutions in water. The concentrations varied between $0.028-0.73 \mathrm{mM}(0.47$ and $1.41 \mathrm{~T})$ and $0.22-0.98(9.4$ and $14.1 \mathrm{~T})$ whereas for the free chelate five solutions were used varying in concentration from $1-5 \mathrm{mM}$ at all four fields studied $(0.47,1.41$ 9.4 and $14.1 \mathrm{~T}$ ). The factory installed inversion recovery pulse sequence was used with the following parameters: recycle delay $\geq 10 \times T_{1}$; number of data points $=16$; number of averages $=4$; inversion pulse delay times for GdDOTP ${ }^{5-}$ ranged from $1-4000 \mathrm{~ms}(0.47$ and $1.41 \mathrm{~T})$ and $10-5000 \mathrm{~ms}(9.4$ and $14.1 \mathrm{~T})$. For NACs the inversion pulse delay times ranged from $12.5-4000 \mathrm{~ms}(0.47$ and $1.41 \mathrm{~T})$ and $10-10000 \mathrm{~ms}(9.4$ and $14.1 \mathrm{~T})$. Relaxation time constants were determined by averaging the values obtained from three measurements for each concentration. 
7.6.5. Chapter 6. Effect of hydration state (q) on rotating frame longitudinal relaxivity $\left(r_{1 \rho}\right)$

NMRD profiles were generated from laboratory and rotating frame relaxivity determinations of each chelate investigated (MnEDTA, $\mathrm{MnCl}_{2}$, GdTTHA ${ }^{3-}$, GdDTPA and $\left.\mathrm{GdCl}_{3}\right)$ in PBS pH 7.4 at the four magnetic field strengths studied $(0.47,1.41,9.4$ and 14.1 T). Relaxivities were determined by linear regression analysis of the longitudinal relaxation rate constants as a function of contrast agent concentration in Excel.

Rotating and laboratory frame data for GdDTPA (Magnevist) were obtained from previous experiments for which experimental details have been described vide supra (7.6.3).

Rotating frame longitudinal relaxation rate constants $\left(R_{1 \rho}\right)$ were determined on five solutions of MnEDTA, $\mathrm{MnCl}_{2}$ and $\mathrm{GdCl}_{3}$ varying in concentration from $5-25 \mathrm{mM}(0.47$ and $1.41 \mathrm{~T})$ and $1-5 \mathrm{mM}(9.4$ and $14.1 \mathrm{~T})$ with the exception of $\mathrm{GdCl}_{3}$ at $14.1 \mathrm{~T}$ which was measured from four solutions. GdTTHA ${ }^{3-} R_{1 \rho}$ values were obtained from a single point determination with solution concentrations of $80.53 \mathrm{mM}(0.47$ and $1.41 \mathrm{~T})$ and 4.026 $\mathrm{mM}(9.4$ and $14.1 \mathrm{~T})$. The factory installed spin locking pulse sequence was employed with the following pulse sequence parameters; recycle delay $\geq 10 \times T_{1}$; number of data points $=16$; number of averages $=4$. Spin locking pulse durations for MnEDTA, $\mathrm{MnCl}_{2}$ and $\mathrm{GdCl}_{3}$ ranged from 0.5 to $40 \mathrm{~ms}(0.47$ and $1.41 \mathrm{~T})$ and $1-400 \mathrm{~ms}(9.4$ and 14.1 T) and the Rabi frequency $\left(\omega_{1}\right)=35 \mathrm{kHz}(0.47$ and $1.41 \mathrm{~T})$ and 6924.2 and 17237.0 
$\mathrm{kHz}$ (9.4 and $14.1 \mathrm{~T}$ respectively). For GdTTHA ${ }^{3-}$ the spin locking pulse durations ranged from 0.5 to $40 \mathrm{~ms}(0.47$ and $1.41 \mathrm{~T})$ and $5-1500 \mathrm{~ms}(9.4$ and $14.1 \mathrm{~T})$ and the Rabi frequency $\left(\omega_{1}\right)=35 \mathrm{kHz}(0.47$ and $1.41 \mathrm{~T})$ and 6924.2 and $17237.0 \mathrm{kHz}(9.4$ and 14.1 T respectively). Relaxation time constants were determined by averaging the values obtained from six measurements (three for GdTTHA ${ }^{3-}$ ) for each concentration.

Laboratory frame longitudinal relaxation rate constants $\left(R_{1}\right)$ were determined on five solutions of MnEDTA, $\mathrm{MnCl}_{2}$ and $\mathrm{GdCl}_{3}$ varying in concentration from $1-5 \mathrm{mM}(0.47$, $1.41 \mathrm{~T}, 9.4$ and $14.1 \mathrm{~T}$ ) with the exception of $\mathrm{GdCl}_{3} 14.1 \mathrm{~T}$ which was measured from three solutions. GdTTHA ${ }^{3-} R_{1}$ values were obtained from a single point determination with solution concentrations of $80.53 \mathrm{mM}(0.47$ and $1.41 \mathrm{~T})$ and $4.026 \mathrm{mM}(9.4$ and 14.1 T). The factory installed inversion recovery pulse sequence was used with the following parameters: recycle delay $\geq 10 \times T_{1}$; number of data points $=16$; number of averages $=4$. Inversion pulse delay times for $\mathrm{MnEDTA}, \mathrm{MnCl}_{2}$ and $\mathrm{GdCl}_{3}$ ranged from $1-5000 \mathrm{~ms}$ (0.47 and $1.41 \mathrm{~T})$ and $6-6600 \mathrm{~ms}(9.4$ and $14.1 \mathrm{~T})$. For GdTTHA ${ }^{3-}$ the inversion pulse delay times ranged from $1-5000 \mathrm{~ms}(0.47$ and $1.41 \mathrm{~T})$ and $10-3000 \mathrm{~ms}(9.4$ and 14.1 T). Relaxation time constants were determined by averaging the values obtained from six measurements (three for GdTTHA ${ }^{3-}$ ) for each concentration. 


\section{COMPLETE LIST OF REFERENCES}

(1) Rabi, I. I.; Zacharias, J. R.; Millman, S.; Kusch, P., A New Method of Measuring Nuclear Magnetic Moment. Phys Rev 1938, 53 (4), 318-327.

(2) Purcell, E. M.; Torrey, H. C.; Pound, R. V., Resonance absorption by nuclear magnetic moments in a solid. Phys Rev 1946, 69, 37-38.

(3) Bloch, F.; Hansen, H. H.; Packard, M., Nuclear Induction. Physical Review 1946, 69, 127.

(4) Lauterbur, P. C., Image Formation by Induced Local Interactions: Examples of Employing Nuclear Magnetic Resonance. Nature 1973, 242 (5394), 190-191.

(5) Mansfield, P.; Maudsley, A. A., Medical imaging by NMR. Br J Radiol 1977, 50, 188-194.

(6) Damadian, R.; Goldsmith, M.; Minkoff, L., NMR in cancer: XVI. Fonar image of live human-body. Physiolog Chem Phys 1977, 9 (108), 97-100.

(7) Blamire, A. M., The technology of MRI - the next 10 years? . Br J Radiol 2008, 81, 601-617.

(8) 2007 MRI Market Summary Report. IMV, Ltd.: 2007.

(9) Kwong, R. Y.; Yucel, E. K., Cardiology patient pages. Computed tomography scan and magnetic resonance imaging. Circulation 2003, 108, 104-106.

(10) Tatlisumak, T., Is CT or MRI the method of choice for imaging patients with acute stroke? Why should men divide if fate has united? Stroke 2002, 33, 2144-2145.

(11) Gupta, R. T.; Kauffman, C. R.; Polascik, T. J.; Taneja, S. S.; Rosenkrantz, A. B., The state of prostate MRI in 2013. Oncology- $N y$ 2013, 27, 262-270.

(12) Li, X.; Priest, R. A.; Woodward, W. J.; Siddiqui, F.; Beer, T. M.; Garzotto, M. G.; Rooney, W. D.; Jr, C. S. S., Cell membrane water exchange effects in prostate DCEMRI. Journal of Magnetic Resonance 2012, 218, 77-85.

(13) Li, X.; Priest, R. A.; Woodward, W. J.; Tagge, I. J.; Siddiqui, F.; Huang, W.; Rooney, W. D.; Beer, T. M.; Garzotto, M. G.; Jr, C. S. S., Feasibility of shutter-speed 
DCE-MRI for improved prostate cancer detection. Magnetic Resonance in Medicine 2013, 69, 171-178.

(14) Rooney, W. D.; Johnson, G.; Li, X.; Cohen, E. R.; Kim, S.-G.; Ugurbil, K.; Springer Jr, C. S., Magnetic Field and Tissue Dependencies of Human Brain Longitudinal ${ }^{1} \mathrm{H}_{2} \mathrm{O}$ Relaxation in Vivo. Magnetic Resonance in Medicine 2007, 57, 308318.

(15) Tieleman, A.; Vandemaele, P.; Seurinck, R.; Deblaere, K.; Achten, E., Comparison between functional magnetic resonance imaging at 1.5 and 3 tesla - effect of increased field strength on 4 paradigms used during presurgical work-up. Invest Radiol 2007, 42, 130-138.

(16) Levitt, M. H., Spin Dynamics: Basics of Nuclear Magnetic Resonance. Second ed.; John Wiley \& Sons Ltd: United Kingdom, 2008.

(17) Vold, R. L.; Waugh, J. S.; Klein, M. P.; Phelps, D. E., Measurement of spin relaxation in complex systems. $J$ Chem Phys 1968, 48, 3831-3832.

(18) Meiboom, S.; Gill, D., Modified spin-echo method for measuring nuclear relaxation times. Rev Sci Instr 1958, 29 (8), 688-691.

(19) Carr, H. Y.; Purcell, E. M., Effects of diffusion on free precession in nuclear magnetic resonance experiments. Phys Rev 1954, 94 (3), 630-638.

(20) Fletcher, L. M.; Barsotti, J. B.; Hornak, J. P., A Multispectral Analysis of Brain Tissues. Magn. Reson. Med. 1993, 29, 623-630.

(21) Hammer, M. MRI Physics: Tissue Contrast in MRI. http://www.xrayphysics.com/ (accessed 05/15/2015).

(22) Aime, S.; Botta, M.; Fasano, M.; Terreno, E., Lanthanide(III) chelates for NMR biomedical applications. Chemical Society Reviews 1998, 27, 19-29.

(23) Woods, M.; Zhang, S.; Kovacs, Z.; Sherry, D., Advances in the design of responsive and targeted lanthanide complexes as diagnostic and therapeutic agents. Advances in Supramolecular Chemstry 2003, 9, 1-38. 
(24) Woods, M.; Zhang, S.; Dean, S., Toward the design of MR agents for imaging $\beta$ cell function. Current Medicinal Chemistry: Immunology, Endocrine \& Metabolic Agents 2004, 4 (4), 349-369.

(25) Woods, M.; Woessner, D. E.; Sherry, D., Paramagnetic lanthanide complexes as PARACEST agents for medical imaging. Chem Soc Rev 2006, 35 (6), 500-511.

(26) Sherry, D.; Zhang, S.; Woods, M., Water exchange is the key parameter in the design of next-generation MRI agents. Medicinal Inorganic Chemistry 2005, 903 (151165).

(27) Kalman, F. K.; Woods, M.; Caravan, P.; Jurek, P.; Spiller, M.; Tircso, G.; Kiraly, R.; Brucher, E.; Sherry, D., Potentiometric and Relaxometric Properties of a GadoliniumBased MRI Contrast Agent for Sensing Tissue pH. Inorg. Chem. 2007, 46, 5260-5270.

(28) Caravan, P.; Ellison, J.; McMurray, T. J.; Lauffer, R. B., Gadolinium(III) Chelates as MRI Contrast Agents: Structure, Dynamics, and Applications. Chem Rev 1999, 99, 2293-2352.

(29) Burtea, C.; Laurent, S.; Elst, L. V.; Muller, R. N., Contrast Agents: Magnetic Resonance. In Handbook of Experimental Pharmacology, W, S.; M, S., Eds. SpringerVerlag: Berlin, 2008; Vol. 185, pp 135-165.

(30) Lauffer, R. B., Paramagnetic Metal Complexes as Water Proton Relaxation Agents for NMR Imaging: Theory and Design. Chem Rev 1987, 87, 901-927.

(31) Pascolo, L.; Cupelli, F.; Anelli, P. L.; Lorusso, V.; Visigalli, M.; Uggeri, F.; Tiribelli, C., Molecular Mechanisms for the Hepatic Uptake of Magnetic Resonance Imaging Contrast Agents. Biochem Biophys Res Commun 1999, 257, 746-752.

(32) Aime, S.; Caravan, P., Biodistribution of gadolinium-based contrast agents, including gadolinium deposition. J Magn Reson Imaging 2009, 30 (6), 1259 - 1267.

(33) Rohrer, M.; Bauer, H.; Mintorovitch, J.; Requardt, M.; Weinmann, H. J., Comparison of magnetic properties of MRI contrast media solutions at different magnetic field strengths. Invest. Radiol 2005, 40, 715-724. 
(34) Erno, B.; Dean, S., Stability and toxicity of contrast agents. The chemistry of contrast agents in medical magnetic resonance imaging. Merbach, A. E.; Toth, E., Eds. John Wiley and Sons Ltd: New York, 2001; Vol. 243, pp 243-278.

(35) Raymond, K. N.; Pierre, V., Next Generation, High Relaxivity Gadolinium MRI Agents. Bioconjugate Chem 2005, 16, 3-8.

(36) Tweedle, M. F.; Wedeking, P.; Kumar, K., Biodistribution of radiolabeled, formulated gadopentetate, gadoteridol, gadoterate, and gadodiamide in mice and rats. . Invest Radiol 1995, 30, 372-380.

(37) Wedeking, P.; Kumar, K.; Tweedle, M. F., Dose-dependent biodistribution of $\left.{ }^{[153} \mathrm{Gd}\right] \mathrm{Gd}(\text { acetate })_{\mathrm{n}}$ in mice. . Nucl Med Biol. 1993, 20, 679-691.

(38) Wedeking, P.; Kumar, K.; Tweedle, M. F., Dissociation of gadolinium chelates in mice: relationship to chemical characteristics. . Magn Reson Imaging 1992, 10, 641-648. (39) Gibby, W. A.; Gibby, K. A.; Gibby, W. A., Comparison of Gd DTPA-BMA (Omniscan) versus Gd HPDO3A (ProHance) retention in human bone tissue by inductively coupled plasma atomic emission spectroscopy. . Invest Radiol 2004, 39, 138142.

(40) White, G. W.; Gibby, W. A.; Tweedle, M. F., Comparison of Gd(DTPA-BMA) (Omniscan) versus Gd(HP-DO3A) (ProHance) relative to gadolinium retention in human bone tissue by inductively coupled plasma mass spectroscopy. Invest Radiol 2006, 41, 272-278.

(41) Wedeking, P.; Tweedle, M., Comparison of the biodistribution of ${ }^{153} \mathrm{Gd}$-labeled Gd(DTPA $)^{2-}, \operatorname{Gd}(\mathrm{DOTA})^{-}$, and $\mathrm{Gd}(\text { acetate })_{\mathrm{n}}$ in mice. Int J Rad Appl Instrum B 1988, 15, 395-402.

(42) Solomon, I., Relaxation processes in a system of two spins. Phys Rev 1955, 99, 559565 .

(43) Solomon, I.; Bloembergen, N., Nuclear magnetic interaction in the HF molecules. $J$ Chem Phys 1956, 25, 261-266.

(44) Bloembergen, N., Proton relaxation times in paramagnetic solutions. J Chem Phys 1957, 27, 572-573. 
(45) Bloembergen, N.; Morgan, L. O., Proton relaxation times in paramagnetic solutions. Effects of electron spin relaxation. $J$ Chem Phys 1961, 34, 842-850.

(46) Freed, J. H., Dynamic effects of pair correlation functions on spin relaxation by translational diffusion in liquids. II. Finite jumps and independent $\mathrm{T}_{1}$ processes. $J$ Chem Phys 1978, 68, 4034-4037.

(47) Anderson, A. G.; Redfield, A. G., Nuclear spin-latice relaxation in metals. Physical Review 1959, 116, 583-591.

(48) Rooney, W. D.; Johnson, G.; Li, X.; Cohen, E. R.; Kim, S.-G.; Ugurbil, K.; Springer, C. S., Jr., Magnetic field and tissue dependencies of human brain longitudinal ${ }^{1} \mathrm{H}_{2} \mathrm{O}$ relaxation in vivo. Magn. Reson. Med. 2007, 57 (2), 308-318.

(49) Lauffer, R. B., Paramagnetic metal complexes as water proton relaxation agents for NMR imaging: theory and design. Chem. Rev. 1987, 87 (5), 901-27.

(50) Powell, D. H.; Dhubhghaill, O. M. N.; Pubanz, D.; Helm, L.; Lebedev, Y. S.; Schlaepfer, W.; Merbach, A. E., High-pressure NMR kinetics. Part 74. Structural and Dynamic Parameters Obtained from ${ }^{17} \mathrm{O}$ NMR, EPR, and NMRD Studies of Monomeric and Dimeric $\mathrm{Gd}^{3+}$ Complexes of Interest in Magnetic Resonance Imaging: An Integrated and Theoretically Self-Consistent Approach. J Am Chem Soc 1996, 118 (39), 9333-9346.

(51) Caravan, P., Strategies for increasing the sensitivity of gadolinium based MRI contrast agents. Chemical Society Reviews 2006, 35, 512-523.

(52) Cacheris, W. P.; Quay, S. C.; Rocklage, S. M., The Relationship between Thermodynamics and the Toxicity of Gadolinium Complexes. Magn. Reson. Imaging 1990, 8, 467-481.

(53) Dickins, R. S.; Aime, S.; Batsanov, A. S.; Beeby, A.; Botta, M.; Bruce, J. I.; Howard, J. A. K.; Love, C. S.; Parker, D.; Peacock, R. D.; Puschmann, H., J. Am. Chem. Soc. 2002, 124, 12697-12705.

(54) Datta, A.; Raymond, K. N., Gd-Hydroxypyridinone (HOPO)-Based HighRelaxivity Magnetic Resonance Imaging (MRI) Contrast Agents. Accounts of Chemical Research 2009, 42, 938-947. 
(55) Aime, S.; Calabi, L.; Cavallotti, C.; Gianolio, E.; Giovenzana, G. B.; Losi, P.; Maiocchi, A.; Palmisano, G.; Sisti, M., [Gd-AAZTA] ${ }^{-}$: A New Structural Entry for an Improved Generation of MRI Contrast Agents. Inorganic Chemistry 2004, 43, 75887590.

(56) Tóth, E. v.; Helm, L.; Merbach, A. E., Wiley: New York, 2001; p 45.

(57) Terreno, E.; Castelli, D. D.; Viale, A.; Aime, S., Challenges for molecular magnetic resonance imaging. Chem Rev 2010, 110, 3019-3042.

(58) Werner, E. J.; Datta, A.; Jocher, C. J.; Raymond, K. N., High-relaxivity MRI contrast agents: where coordination chemistry meets medical imaging. Angewandte Chemie International Edition 2008, 47, 8568-8580.

(59) Benmelouka, M.; Borel, A.; Moriggi, L.; Helm, L.; Merbach, A. E., Design of Gd(III)-Based Magnetic Resonance Imaging Contrast Agents: Static and Transient ZeroField Splitting Contributions to the Electronic Relaxation and Their Impact on Relaxivity. The Journal of Physical Chemistry B 2007, 111, 832-840.

(60) Senn, F.; Helm, L.; Borel, A.; Daul, C. A., Electronic fine structure calculation of [Gd(DOTA)(H2O) $]^{-}$using LF-DFT: The zero field splitting. Comptes Rendus Chimie 2012, 15, 250-254.

(61) Powell, D. H.; Ni Dhubhghaill, O. M.; Pubanz, D.; Helm, L.; Lebedev, Y. S.; Schlaepfer, W.; Merbach, A. E., High-pressure NMR kinetics. Part 74. Structural and Dynamic Parameters Obtained from ${ }^{17} \mathrm{O}$ NMR, EPR, and NMRD Studies of Monomeric and Dimeric $\mathrm{Gd}^{3+}$ Complexes of Interest in Magnetic Resonance Imaging: An Integrated and Theoretically Self-Consistent Approach. J. Am. Chem. Soc. 1996, 118 (39), 93339346.

(62) Redfield, A. G., Nuclear magnetic resonance saturation and rotary saturation in solids. Phys Rev 1955, 98, 1787-1809.

(63) Sepponen, R. E.; Pohjonen, J. A.; Sipponen, J. T.; Tanttu, J. I., A method for $\mathrm{T}_{1 p}$ imaging. J Comput Assist Tomogr 1985, 9 (6), 1007-1011. 
(64) Halavaara, J. T.; Sepponen, R. E.; Lamminen, A. E.; Vehmas, T.; Bondestam, S., Spin Lock and Magnetization Transfer MR Imaging of Focal Liver Lesions. Magn. Reson. Imag. 1998, 16 (4), 359-364.

(65) Grohn, O. H. J.; Makela, H. I.; Lukkarinen, J. A.; DelaBarre, L.; Lin, J.; Garwood, M.; Kauppinen, R. A., On- and Off-Resonance $\mathrm{T}_{1 \rho}$ MRI in Acute Cerebral Ischemia of the Rat. Magn. Reson. Med. 2003, 49, 172-176.

(66) Regatte, R. R.; Akella, S. V. S.; Wheaton, A. J.; Borthakur, A.; Kneeland, J. B.; Reddy, R., $\mathrm{T}_{1 \rho}$-Relaxation Mapping of Human Femoral-Tibial Cartilage In Vivo. J. Magn. Reson. Imag. 2003, 18, 336-341.

(67) Borthakur, A.; Wheaton, A. J.; Gougoutas, A. J.; Akella, S. V. S.; Regatte, R. R.; Charagundla, S. R.; Reddy, R., In Vivo Measurement of $\mathrm{T}_{1 \rho}$ Dispersion in the Human Brain at 1.5 Tesla. J. Magn. Reson. Imag. 2004, 19, 403-409.

(68) Borthakur, A.; Sochor, M.; Davatzikos, C.; Trojanowski, J. Q.; Clark, C. M., T1 $\rho$ MRI of Alzheimer's disease. NeuroImage 2008, 41, 1199-1205.

(69) Haris, M.; Singh, A.; Cai, K.; Davatzikos, C.; Trojanowski, J. Q.; Melhem, E. R.; Clark, C. M.; Borthakur, A., $\mathrm{T}_{1 \text { rho }}\left(\mathrm{T}_{1 \rho}\right)$ MR imaging in Alzheimer' disease and Parkinson's disease with and without dementia. J Neurol 2011, 258, 380-385.

(70) Chopra, S.; McClung, R. E. D.; Jordan, R. B., Rotating-Frame Relaxation Rates of Solvent Molecules in Solutions of Paramagnetic Ions Undergoing Solvent Exchange. $J$ Mag Res 1984, 59, 361-372.

(71) Kumar, K.; Jin, T.; Wang, X.; Desreux, J. F.; Tweedle, M. F., Effect of Ligand Basicity on the Formation and Dissociation Equilibria and Kinetics of $\mathrm{Gd}^{3+}$ Complexes of Macrocyclic Polyamino Carboxylates. Inorg. Chem. 1994, 33, 3823-3829.

(72) GmbH, B. O., The minispec: mq series users manual. 2.2 ed.; Germany, 2003.

(73) Celine, H.; Sophie, L.; Luce, V. E., How to perform accurate and reliable measurements of longitudinal and transverse relaxation times of MRI contrast media in aqueous solutions. Contrast Media Mol Imaging 2009, 4, 312-321. 
(74) Avedano, S.; Botta, M.; Haigh, J.; Longo, D.; Woods, M., Coupling fast water exchange to slow molecular tumbling in $\mathrm{Gd}^{3+}$ chelates: why faster is not always better. Inorganic Chemistry 2013, 52 (15), 8436-8450.

(75) Alford Jamu, K.; Rutt Brian, K.; Scholl Timothy, J.; Handler William, B.; Chronik Blaine, A., Delta relaxation enhanced MR: improving activation-specificity of molecular probes through $\mathrm{R}_{1}$ dispersion imaging. Magn Reson Med 2009, 61 (4), 796-802.

(76) Alford, J. K.; Scholl, T. J.; Handler, W. B.; Chronik, B. A., Design and construction of a prototype high-power $\mathrm{B}_{0}$ insert coil for field-cycled imaging in superconducting MRI systems. Concepts Magn. Reson., Part B 2009, 35B (1), 1-10.

(77) Choi, C.-H.; Hutchison, J. M. S.; Lurie, D. J., Design and construction of an actively frequency-switchable RF coil for field-dependent Magnetisation Transfer Contrast MRI with fast field-cycling. J. Magn. Reson. 2010, 207 (1), 134-139.

(78) Hoelscher Uvo, C.; Lother, S.; Fidler, F.; Blaimer, M.; Jakob, P., Quantification and localization of contrast agents using delta relaxation enhanced magnetic resonance at 1.5 T. MAGMA 2012, 25 (3), 223-31.

(79) Lurie, D. J.; Aime, S.; Baroni, S.; Booth, N. A.; Broche, L. M.; Choi, C.-H.; Davies, G. R.; Ismail, S.; O hOgain, D.; Pine, K. J., Fast field-cycling magnetic resonance imaging. Comp. Rend. Phys. 2010, 11 (2), 136-148.

(80) Lurie, D. J.; Davies, G. R.; Pine, K. J. Field-offset coil for use with an open mri scanner. 2013-GB51921, 20130718., 2014.

(81) Santyr, G. E.; Henkelman, R. M.; Bronskill, M. J., Spin locking for magnetic resonance imaging with application to human breast. Magn Reson Med 1989, 12, 25-37.

(82) Richardson, O. C.; Scott, M. L. J.; Tanner, S. F.; Waterton, J. C.; Buckley, D. L., Overcoming the low relaxivity of gadofosveset at high field with spin locking. Magn Reson Med 2012, 68 (4), 1234-8.

(83) Tanttu, J. I.; Sepponen, R. E.; Lipton, M. J.; Kuusela, T., Synergistic enhancement of MRI with Gd-DTPA and magnetization transfer. J Comput Assist Tomogr 1992, 16, $19-24$. 
(84) Aronen, H. J.; Peltonen, T. K.; Tanttu, J. I.; Sipila, L. M.; Ruhala, M. T.; Jaaskelainen, J.; Sepponen, R., Spin-Lock Imaging in Contrast-Enhanced Magnetic Resonance Imaging of Human Gliomas. Acad Radiol 1996, 3, S170-S172.

(85) Aronen, H. J.; Ramadan, U. A.; Peltonen, T. K.; Markkola, A. T.; Tanttu, J. I.; Jaaskelainen, J.; Hakkinen, A.-M.; Sepponen, R. E., 3D spin-lock imaging of human gliomas. Magn Reson Imag. 1999, 17, 1001-1010.

(86) Geraldes, C. F. G. C.; Sherry, A. D.; Brown, R. D., III; Koenig, S. H., Magnetic field dependence of solvent proton relaxation rates induced by gadolinium( $3+$ ) and manganese $(2+)$ complexes of various polyaza macrocyclic ligands: implications for NMR imaging. Magn. Reson. Med. 1986, 3 (2), 242-50.

(87) Laurent, S.; Henoumont, C.; Vander Elst, L.; Muller, R. N., Synthesis and Physicochemical Characterisation of Gd-DTPA Derivatives as Contrast Agents for MRI. Eur. J. Inorg. Chem. 2012, 2012 (12), 1889-1915.

(88) Wilson, G. J.; Woods, M.; Jr, C. S. S.; Bastawrou, S.; Bhargava, P.; Maki, J. H., Human whole-blood $1 \mathrm{H}_{2} \mathrm{O}$ longitudinal relaxation with normal and high-relaxivity contrast reagents: Influence of trans-cell-membrane water exchange. Magn Reson Med 2014, 1746-1754.

(89) Caravan, P.; Cloutier, N. J.; Greenfield, M. T.; McDermid, S. A.; Dunham, S. U.; Bulte, J. W. M.; Jr, J. C. A.; Looby, R. J.; Supkowski, R. M.; Jr, W. D. H.; McMurry, T. J.; Lauffer, R. B., The Interaction of MS-325 with Human Serum Albumin and Its Effect on Proton Relaxation Rates. Journal of the American Chemical Society 2002, 124 (12), 3152-3162.

(90) Bottomley, P. A.; Foster, T. H.; Argersinger, R. E.; Pfeifer, L. M., A review of normal tissue hydrogen NMR relaxation times and relaxation mechanisms from 1-100 MHz: Dependence on tissue type, NMR frequency, temperature, species, excision, and age. Med Phys 1984, 11 .

(91) Bottomley, P. A.; Hardy, C. J.; Argersinger, R. E.; Allen-Moore, G., A review of $1 \mathrm{H}$ nuclear magnetic resonance relaxation in pathology: Are $\mathrm{T}_{1}$ and $\mathrm{T}_{2}$ diagnostic? Med Phys 1987, 14 (1), 1-37. 
(92) Hocq, A.; Luhmer, M.; Saussez, S.; Louryan, S.; Gillis, P.; Gossuin, Y., Effect of magnetic field and iron content on NMR proton relaxation of liver, spleen and brain tissues. Contrast Media Mol. Imaging 2015, 10, 144-152.

(93) Plush, S. E.; Woods, M.; Zhou, Y.-F.; Kadali, S. B.; Wong, M. S.; Sherry, D., Nanoassembled Capsules as Delivery Vehicles for Large Payloads of High Relaxivity $\mathrm{Gd}^{3+}$ Agents. J. AM. CHEM. SOC. 2009, 131, 15918-15923.

(94) Aime, S.; Botta, M.; Crich, S. G.; Giovenzana, G. B.; Pagliarin, R.; Piccinini, M.; Sisti, M.; Terreno, E., Towards MRI contrast agents of improved efficacy. NMR relaxometric investigations of the binding interaction to HSA of a novel heptadentate macrocyclic triphosphonate Gd(III)-complex. J. Biol. Inorg. Chem. 1997, 2 (4), 470-479.

(95) Chan, K. W.-Y.; Wong, W.-T., Small molecular gadolinium(III) complexes as MRI contrast agents for diagnostic imaging. Coord. Chem. Rev. 2007, 251 (17-20), 2428-2451. (96) Lauterburg, P. C.; Dias, M. H. M.; Rubin, A. M., Frontiers of Biological Energetics. Academic Press, New York, 1978.

(97) Aoki, I.; Tanaka, C.; Takegami, T.; Ebisu, T.; Umeda, M.; Fukunaga, M.; Fukuda, K.; Silva, A. C.; Koretsky, A. P.; Naruse, S., Dynamic activity-induced manganesedependent contrast magnetic resonance imaging (DAIM MRI). Magn. Reson. Med. 2002, 48, 927-933.

(98) Crossgrove, J.; Zheng, W., Manganese toxicity upon overexposure. NMR Biomed. 2004, 17, 544-553.

(99) Pautler, R. G., In vivo, trans-synaptic tract-tracing utilizing manganese-enhanced magnetic resonance imaging (MEMRI). NMR Biomed. 2004, 17, 595-601.

(100) Aschner, M.; Guilarte, T. R.; Schneider, J. S.; Zheng, W., Manganese: Recent advances in understanding its transport and neurotoxicity. Toxicol. Appl. Pharmacol. 2007, 221, 131-147.

(101) Gale, E. M.; Zhu, J.; Caravan, P., Direct Measurement of the Mn(II) Hydration State in Metal Complexes and Metalloproteins Through ${ }^{17} \mathrm{O}$ NMR Linewidths. $J$ Am Chem Soc. 2013, 135 (49), 18600-18608.

(102) Ailion, D. C., NMR and ultraslow motions. Adv. Mag. Res. 1971, 5, 177-227. 
(103) Koenig, S. H.; Brown, R. D., Relaxation of Solvent Protons by Paramagnetic Ions and Its Dependence on Magnetic Field and Chemical Environment: Implications for NMR Imaging. Magnetic Resonance in Medicine 1984, 1, 478-495.

(104) Koenig, S. H.; Brown, R. D.; Studebaker, J. In Quant. Biol., Cold Spring Harbor Symp., 1971; p 551.

(105) Idée, J.-M.; Port, M.; Medina, C.; Lancelot, E.; Fayoux, E.; Ballet, S.; Corot, C., Possible involvement of gadolinium chelates in the pathophysiology of nephrogenic systemic fibrosis: A critical review. Toxicology 2008, 248, 77-88.

(106) Sieber, M. A.; Steger-Hartmann, T.; Lengsfeld, P.; Pietsch, H., Gadolinium-based contrast agents and NSF: evidence from animal experience. J. Magn. Reson. Imag. 2009, 30 (6), 1268-1276.

(107) Chen, J. W.; Belford, R. L.; Clarkson, R. B., Second-Sphere and Outer-Sphere Proton Relaxation of Paramagnetic Complexes: From EPR to NMRD. J. Phys. Chem. A. 1998, 102, 2117-2130. 
APPENDIX: $T_{1}$ AND $T_{1 p}$ TIME CURVES AT $1.41 \mathrm{~T}(60 \mathrm{MHz})$ AND $37{ }^{\circ} \mathrm{C}$ (APPENDIX TO CHAPTER 2).

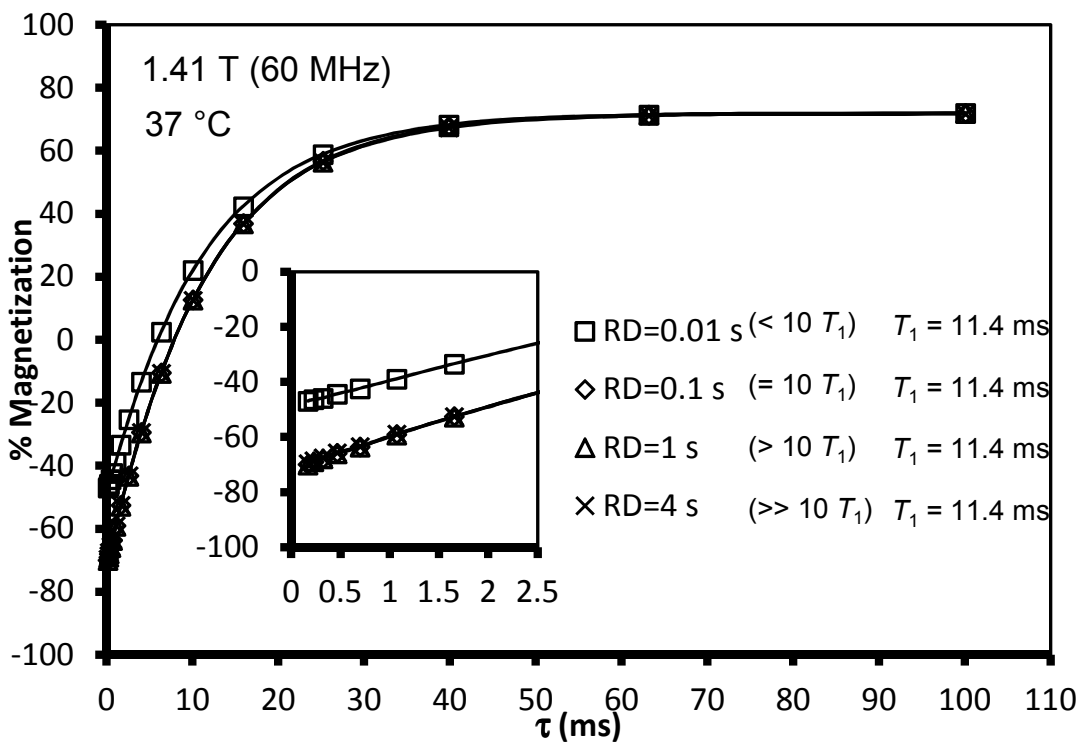

Figure A1.1: Effect of delibarate variations to the relaxation delay (RD) time on $T_{1}$ time curves (inset shows deviation (squares) when relaxation delay is $<10 \times \mathrm{T}_{1}$ ). Sample: PBS $\mathrm{pH} 7.4$ solution of Dotarem, $\left[\mathrm{Gd}^{3+}\right]=25 \mathrm{mM} . \mathrm{n}=1$. 

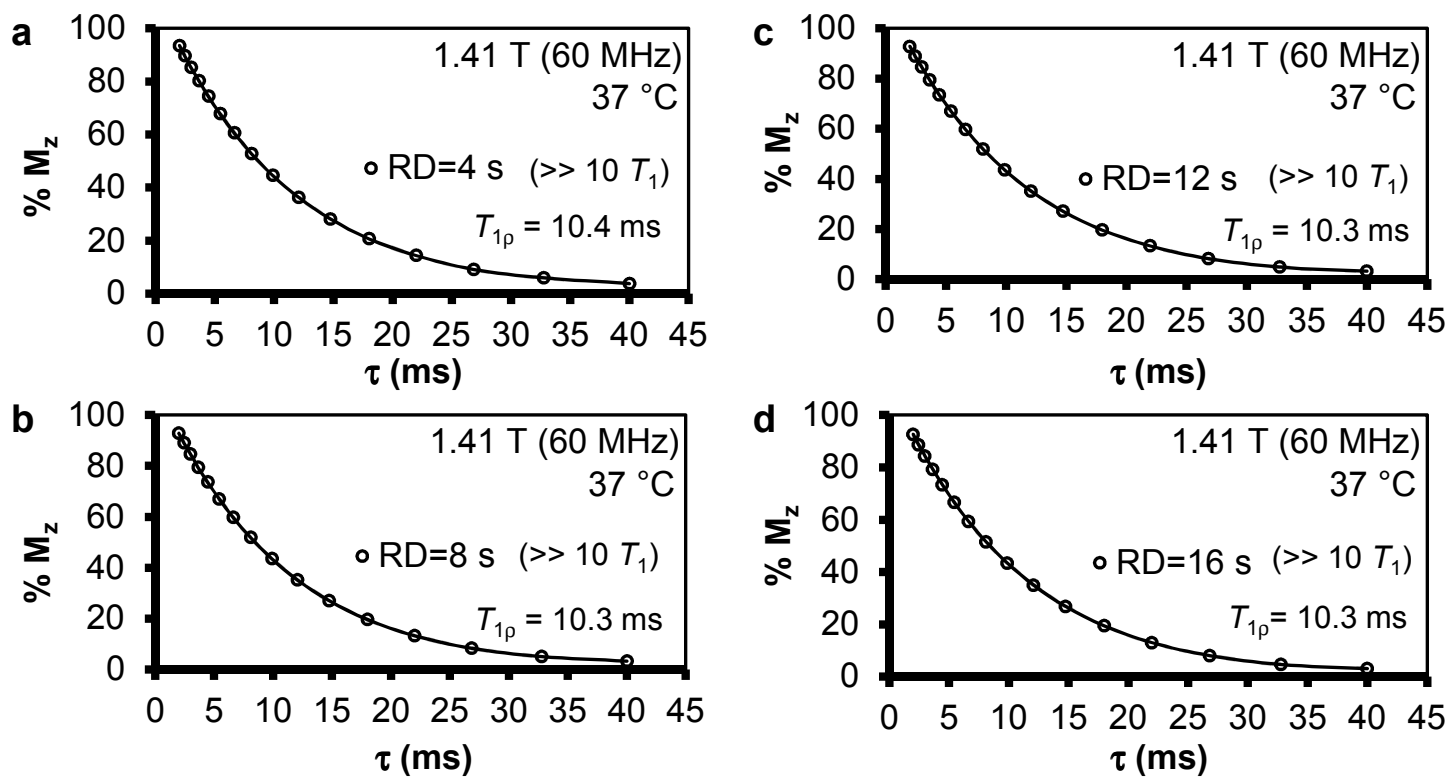

Figure A1.2: Effect of deliberate variations to the relaxation delay (RD) time on $T_{1 p}$ time curves. Sample: PBS pH 7.4 solution of Dotarem, $\left[\mathrm{Gd}^{3+}\right]=25 \mathrm{mM} . \mathrm{n}=1$. 

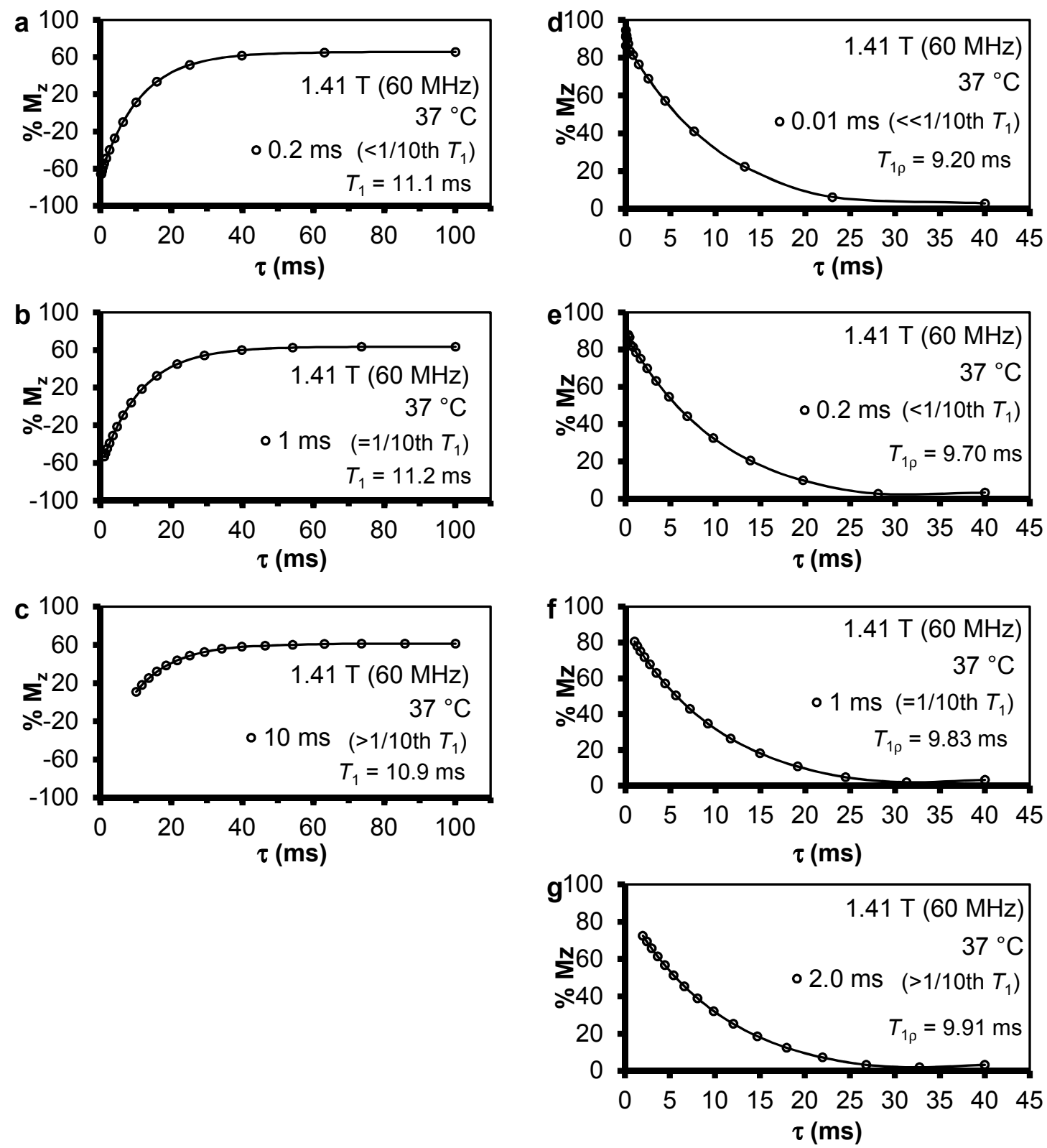

Figure A1.3: Effect of deliberate variations to the first pulse delay time $(\tau)$ on $T_{1}(\mathrm{a}-\mathrm{c})$ and $T_{1 \rho}$ (d $-\mathrm{g}$ ) time curves. Sample: PBS pH 7.4 solution of Dotarem, $\left[\mathrm{Gd}^{3+}\right]=25 \mathrm{mM} . \mathrm{n}=1$. 

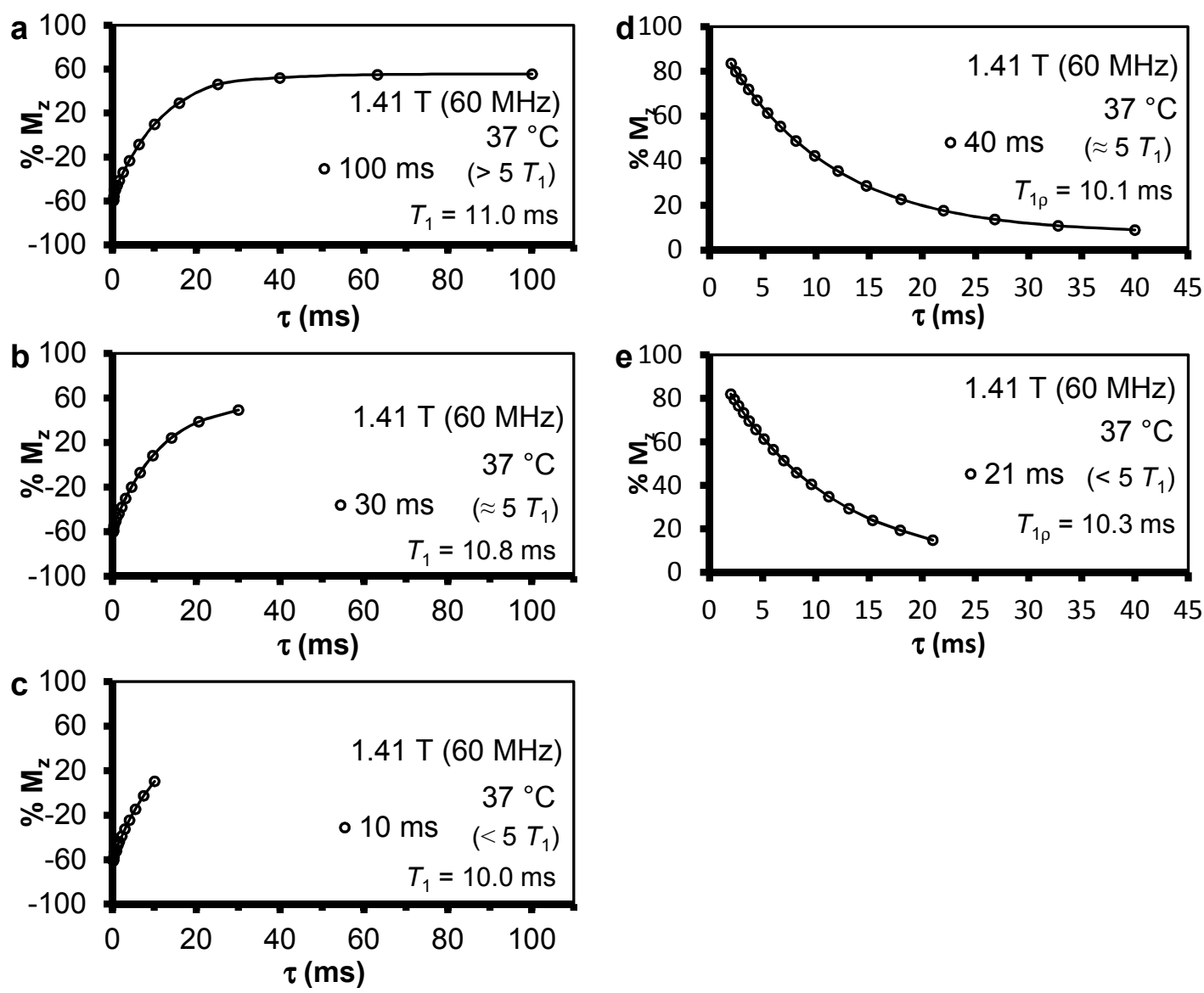

Figure A1.4: Effect of deliberate variations to the last pulse delay time $(\tau)$ on $T_{1}(\mathrm{a}-\mathrm{c})$ and $T_{1 \mathrm{p}}(\mathrm{d}$ $-\mathrm{e})$ time curves. Sample: PBS pH 7.4 solution of Dotarem, $\left[\mathrm{Gd}^{3+}\right]=25 \mathrm{mM} . \mathrm{n}=1$. 

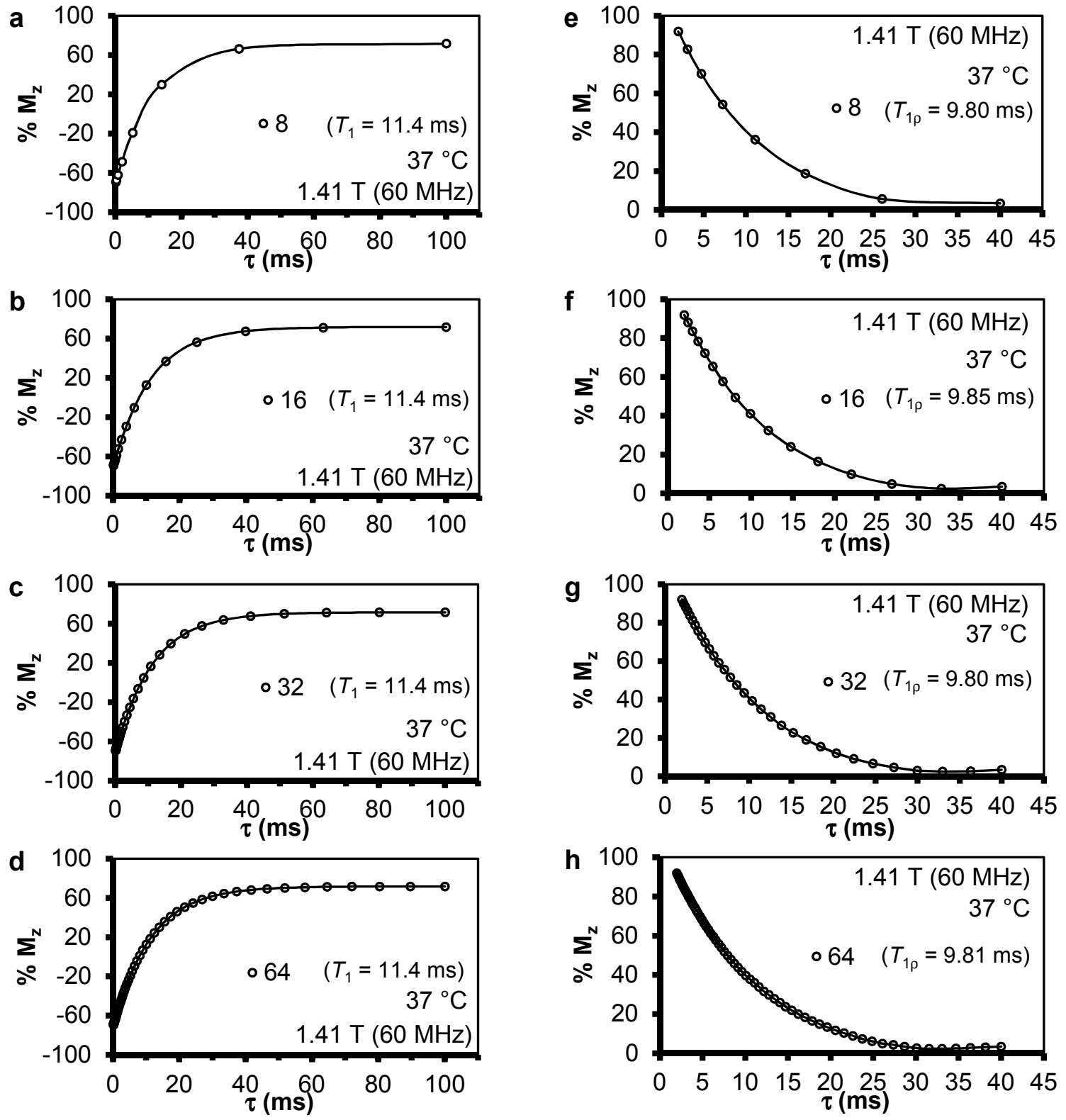

Figure A1.5: Effect of deliberate variations to the number of data points on $T_{1}(a-d)$ and $T_{1 p}(e-$ h) time curves. Sample: PBS pH 7.4 solution of Dotarem, $\left[\mathrm{Gd}^{3+}\right]=25 \mathrm{mM} . \mathrm{n}=1$. 